\title{
METABOLIC STRESS AND ANTIPSYCHOTIC TREATMENT INDUCED GENE EXPRESSION ALTERATIONS \\ IN HUMAN CELL CULTURES \\ $\mathrm{PhD}$ Thesis
}

Sára Kálmán MD

Tutors:

Prof. Károly Mirnics MD, PhD, DSc

Prof. Zoltán Janka MD, PhD, DSc

University of Szeged

Department of Psychiatry

Szeged

2016 


\section{PUBLICATIONS RELATED TO THE DISSERTATION}

I. Kálmán S, Garbett KA, Vereczkei A, Shelton RC, Korade Z, Mirnics K. Metabolic stress-induced microRNA and mRNA expression profiles of human fibroblasts. Exp Cell Res. 2014. 320(2):343-53.

IF $_{2014}: 3.25$

II. Garbett KA, Vereczkei A, Kálmán S, Brown JA, Taylor WD, Faludi G, Korade Ž, Shelton RC, Mirnics K. Coordinated messenger RNA/microRNA changes in fibroblasts of patients with major depression. Biol Psychiatry. 2015. 77(3):256-65.

IF $_{2015}: 10.25$

III. Garbett KA, Vereczkei A, Kálmán S, Wang L, Korade Ž, Shelton RC, Mirnics K. Fibroblasts from patients with major depressive disorder show distinct transcriptional response to metabolic stressors. Transl Psychiatry. 2015. 5:e523.

IF $_{2015}: 5.62$

IV. Kálmán S, Hathy E, Réthelyi JM. A dishful of a troubled mind: induced pluripotent stem cells in psychiatric research. Stem Cells Int. 2016. 2016:7909176.

IF $_{2015}: 2.81$

V. Kálmán S, Garbett KA, Janka Z, Mirnics K. Human dermal fibroblasts in psychiatry research. Neuroscience. 2016. 320:105-21.

IF $_{2015}: 3.36$

Cumulative impact factor: 25.29 


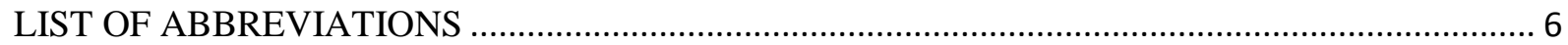

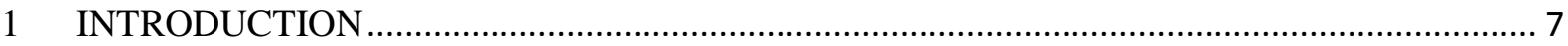

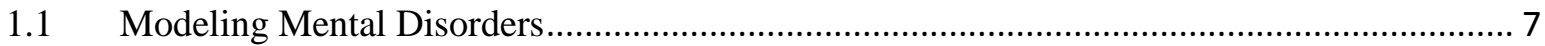

1.2 Study 1 - Diathesis-Stress in Fibroblasts ........................................................................... 7

1.2.1 Human dermal fibroblasts in psychiatric research ................................................ 8

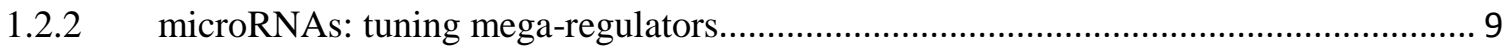

1.3 Study 2 - A Psychopharmacological Assay ....................................................................... 10

1.3.1 Human induced pluripotent and neural cells in psychiatric research ........................... 10

1.3.2 Modeling hippocampal neurogenesis ........................................................................ 11

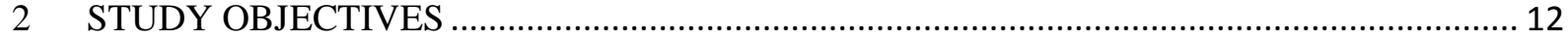

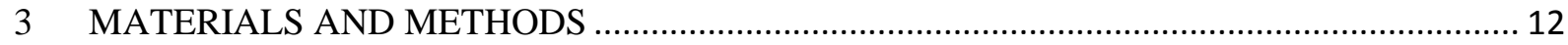

3.1 Study 1 - Diathesis-Stress in Fibroblasts ...................................................................... 12

3.1.1 Skin biopsy and fibroblast cultures ..................................................................... 12

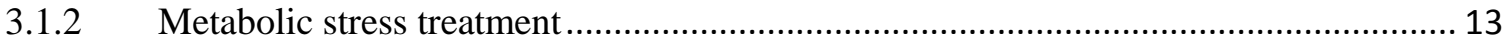

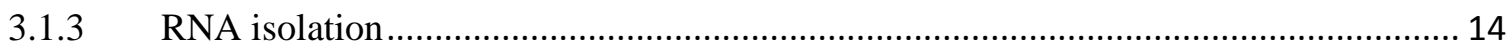

3.1.4 mRNA expression assessment with DNA microarray................................................... 14

3.1.1 mRNA profiling data validation by quantitative polymerase chain reaction ................ 15

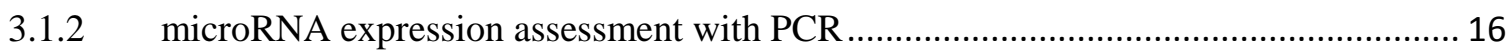

3.1.3 microRNA data validation by individual PCR ….................................................... 16

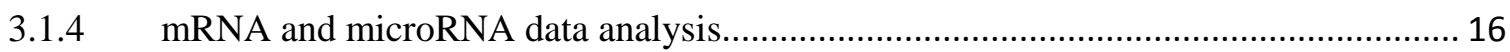

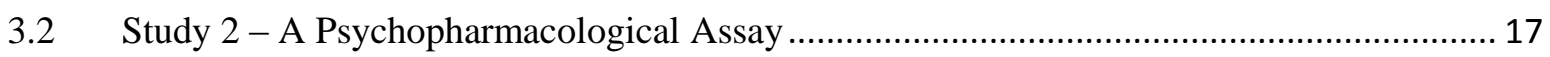

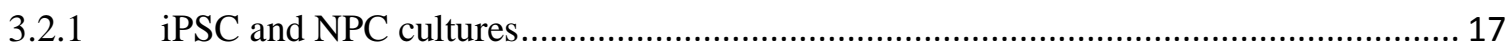

3.2.2 Neural differentiation and treatment with antipsychotics........................................... 18

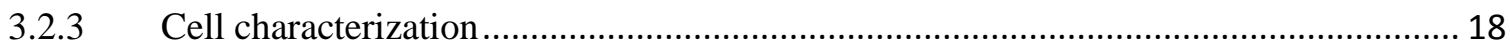

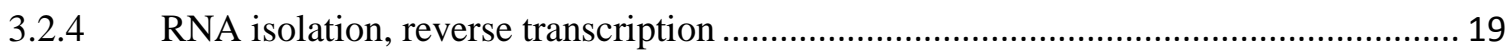

3.2.5 Real-time quantitative PCR and data analysis........................................................... 20

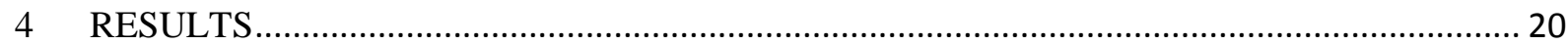

4.1 Study 1 - Diathesis-Stress in Fibroblasts ........................................................................ 20

4.1.1 How fibroblasts adapt on transcriptome-level? .............................................................. 20

4.1.2 Manifestation of MD diathesis in fibroblasts ............................................................... 23

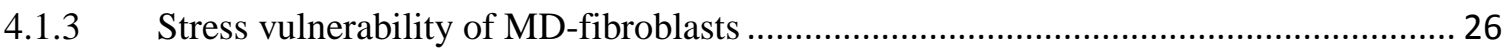

4.2 Study 2 - A Psychopharmacological Assay ........................................................................ 29

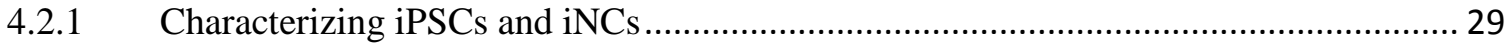




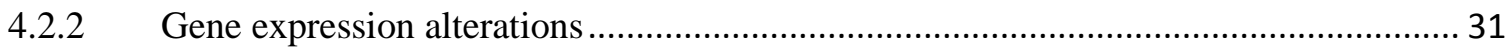

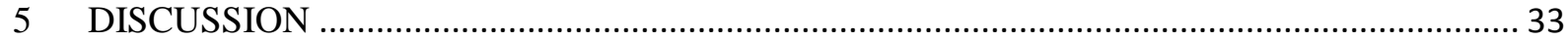

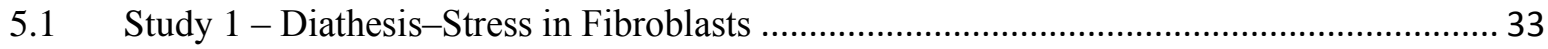

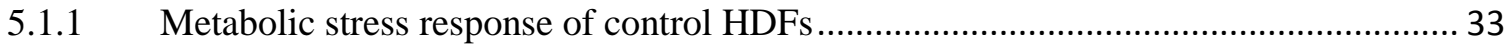

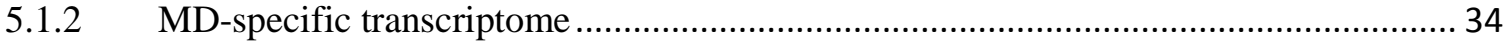

5.1.3 Metabolic stress response of MD fibroblasts ................................................................ 36

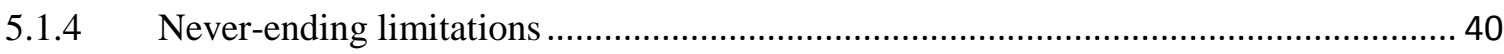

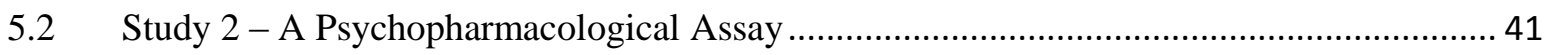

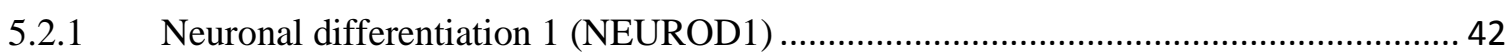

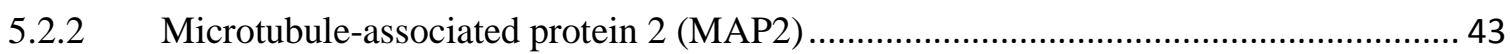

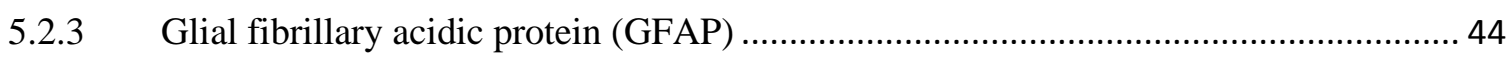

5.2.4 Metabotropic glutamate receptors (mGluR2 and mGluR7) ............................................ 46

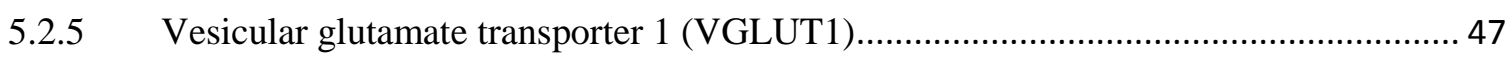

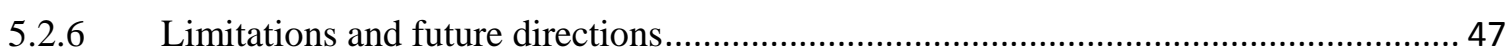

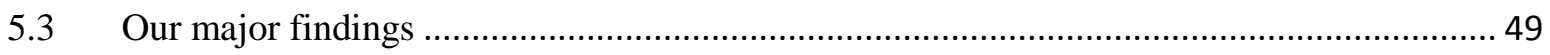

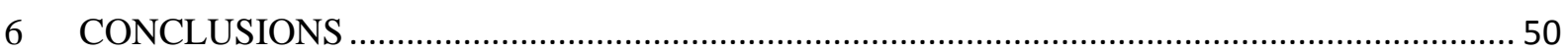

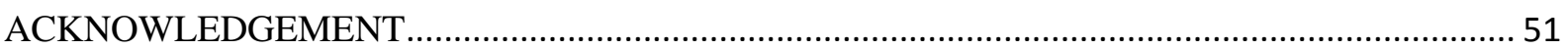

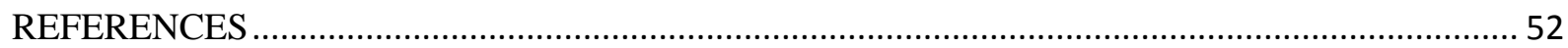




\section{LIST OF ABBREVIATIONS}

AAP: atypical antipsychotic drug

ALR: average logarithmic ratio

AP: antipsychotic drug

CNS: central nervous system

CNT: control

DG: dentate gyrus of the hippocampus

DMEM: Dulbecco's modified Eagle medium

DMSO: dimethyl sulfoxide

FBS: fetal bovine serum

$\mathrm{G} * \mathrm{E}$ : gene-environment interaction

GAL: galactose-enriched, glucose-deprived medium

GFAP: glial fibrillary acidic protein

HDF: human dermal fibroblast

HL: haloperidol

HPA: hypothalamic-pituitary-adrenal axis

iNC: induced pluripotent stem cell-derived neural cell

iPSC: induced pluripotent stem cell

MAP2: microtubule associated protein 2

MD: major depression

mGluR2 and mGluR7: metabotropic glutamate receptor 2 and 7

NEUROD1: Neural differentation 1

NPC: neural progenitor

OL: olanzapine

PBS: phosphate-buffered saline

qPCR: quantitative polymerase chain reaction

RL: lipid-reduced medium

$\mathrm{RP}$ : risperidone

STD: standard

TAP: typical antipsychotic drug

VGLUT1: vesicular glutamate transporter 1 


\section{INTRODUCTION}

\subsection{Modeling Mental Disorders}

As mental disorders are exclusively human conditions and their pathophysiology can not be directly analyzed in the dysfunctioning organ in vivo, investigating and modeling these conditions raises several problems and necessitates compromises. Animal models provide valuable information on the behavioral disturbances and associated structural and functional brain alterations but obviously have their limitations and validation difficulties ${ }^{1}$. Hence, human biospecimens and experimental model systems are indispensable in studying complex, multifactorial neuropsychiatric disorders.

Postmortem observations might be biased by various artifacts related to in vivo effects, applied treatment, cause of death or postmortem interval ${ }^{2}$. Brain banks provide great sample sizes, standardized methodology and detailed clinical information. However, they cannot counterveil that postmortem samples are not appropriate for functional assays evaluation of gene * environment $(\mathrm{G} * \mathrm{E})$ interactions, maladaptation, or drug response and have limited utility in establishing diagnosis and aiding therapeutic approaches which would be one of the greatest clinical advantages of etiology and biomarker studies.

Thus, high effort is taken to develop easy-to-gain and -maintain living subjects for functional, translational and heritability studies in psychiatric disorders. Considering that molecular abnormalities, contributing to neural dysfunction (e.g. mitochondrial, metabolic changes, antioxidant potential, intracellular signaling, geneexpression regulation) are common among cell types and the majority of psychiatric disorders have a heritability between 40 $90 \%$, they can be studied in vitro ${ }^{3}$. Patient derived cellular functional assays enable a systemoriented view, in which mental disorders are the manifestation of the donor's individual genetics and perform functional assays to map $\mathrm{G} * \mathrm{E}$ and $\mathrm{G} * \mathrm{G}$ interactions.

In my thesis, I introduce two research projects which aimed to examine the diathesis-stress model of depression in patient-derived dermal fibroblast cultures (Study 1) and the effect of antipsychotic drugs on induced pluripotent stem cell (iPSC) -derived differentiating neurons (Study 2).

\subsection{StUdy 1 - DiATHESIS-STRESS IN FibROBlastS}

Selye suggested that stress response follows an evolutionary conserved scheme via activating the neuro-immuno-endocrine system, independently from the source or parameters of the stressor ${ }^{4}$. After danger passes, the reaction settles down and the body starts to restore 
the reserves. Yet in some cases, the same external stimulus provokes prolonged, inadequate stress response and results in maladaptive disorders, such as major depression (MD).

In 2012, MD was the leading cause of disability worldwide $\left(\mathrm{WHO}^{5}\right)$ affecting almost every 5th people. It is a multifactorial disorder, with a heritability of 36-70\%, suggesting complex (epi)genome * environment interactions in the background ${ }^{6}$. Despite decades of active research, the exact pathomechanism of MD remains unclear. Candidate gene, linkage and genome-wide association (GWA) studies failed to identify the genetic background of the disorder ${ }^{7,8}$ underpinning the polygenic heterogeneity model. According to this, common genetic variants alone cause minor phenotypic changes, but in certain constellations, their effects sum up and results in decreased individual resilience ${ }^{9,10,11}$. The diathesis-stress model claims that this genetic sensitivity interacts with the environment and may result in the development or remission of clinical symptoms through the life $9^{10}$. Thus, genetic load often manifests only when a stress occurs and anomalies of the adaptation system are heightened.

This $G^{*} E$ based approach of MD is supported by clinical data: there is a well-known association between stressful life-events and the development of depressive symptoms. Chronic stress disposes to immunological and mental disturbances and the inflammatory response modulates the hypothalamic-pituitary-adrenal axis (HPA axis), affective and cognitive functioning. Therefore, HPA-axis and inflammatory system dysregulation are the most studied and proved somatic factors of $\mathrm{MD}^{12,13,14}$. The theory is also plausible in a neurobiological contexts, described in the inflammation-neurodegeneration model of MD.

$\mathrm{MD}$ is also referred to as metabolic syndrome type II, a complex endocrine - metabolic disorder. Stress hormones, vasopressin, enteroendocrine factors (insulin-like growth factor, ghrelin, cholecystokinin) show significant alterations in MD patients ${ }^{15}$. Furthermore, metabolic dysregulation might be associated with depressed mood and cognitive impairment ${ }^{16}$. Thus, it is plausible that $\mathrm{G} * \mathrm{E}$ caused cellular malfunction manifests not only in affective/cognitive disturbances in the CNS, but in immune and metabolic alterations in the peripheral tissues also.

\subsubsection{Human dermal fibroblasts in psychiatric research}

In the recent 5 years, there has been growing literature on human dermal fibroblasts (HDFs) as potential model systems or biomarker resources in psychiatric disorders. They were used to investigate disease-related metabolism, redox homeostasis, membrane transport, apoptotic susceptibility, transcriptome, circadian rhythm or pharmacology in schizophrenia, attention deficit and hyperactivity disorder, MD, and bipolar depression. 
HDFs are relatively easy to obtain from minimal invasive skin biopsies ${ }^{17}$. Their maintenance does not require significant resources and they can be easily propagated for long periods of time without genetic manipulation or external growth factors ${ }^{18}$. The cultures consist of mitotic and post-mitotic, uniform fibroblast cells ${ }^{19}$ and retain genetic stability for 15-20 passages $^{20}$. After 4-5 passages, cell lines thought to be free of pre-sampling in vivo effects, such as health state, diet, medication and environmental factors ${ }^{21,22}$. The gene expression, signaling pathways and receptor profile of fibroblasts are similar to cells of neuro-ectodermal origin $^{23}$. Moreover, they proved to express neuron and glia specific markers ${ }^{24}$.

All these considerations make HDF cultures an appealing, cost-effective experimental model for studying neuropsychiatric disorders. They can be used for directly studying the naive pathophysiology, maladaptation and epi(genetic) regulation; and may be potential subjects of controlled, reproducible, 'individualized' in vitro experiments with the patient's genetic background.

\subsection{2 microRNAs: tuning mega-regulators}

Adaptation requires a quick, accurate, coordinated reaction on systemic and cellular level. MicroRNA-mediated transcription regulation meets these criteria. MicroRNAs are small (18-24-nucleotid-long), single-stranded, non-coding RNAs, key regulators of gene expression. More than 2000 mature microRNAs modulate nearly 60\% of the human genes with a high degree of pleiotropy and functional redundancy. They dampen the gene expression and suppress its intrinsic variations to help the maintenance of homeostasis ${ }^{25}$. Intriguingly, growing body of evidence indicates that microRNAs may exert paracrine and endocrine-like effects after secreted into the interstitium and various biospecimens (e.g. plasma, saliva, tear, or urine) ${ }^{26,27,28}$.

MicroRNAs link to a complementary sequence in the 3 '-untranslated region (UTR) and evoke the degradation, destabilization, storage or transport of the target mRNA, thus induce the permanent or temporary repression of local or cell-wide protein synthesis. However, the presence of a microRNA is not automatically enough to predict its silencing effects. For instance, if the complementarity between the microRNA and the target site is not perfect, the partial hybridization (temporalily) prevents translation but does not lead to mRNA decompose $^{29}$. After the detrimental effect ceases, mRNAs are rehabilitated from the stress granules and translated for rapid recovery ${ }^{30}$. Moreover, recent observations suggest that miRNAs do not have exclusively negative effect on translation: the target genes can be enhanced by direct gene activation, mRNA stabilization, or mRNA de-repression ${ }^{31,32}$. Thus, 
microRNA-driven mRNA metabolism is essential in the protection of the future enzyme sets and reserve capacities ${ }^{33}$. This remarkable dynamicity and complexity enables microRNAs to fine-tune the overall cell transcriptome or local protein sets (e.g. during synapse formation).

Fifty percent of the known human microRNAs are expressed in the $\mathrm{CNS}^{34}$. Some microRNAs show restricted tissue distribution but most of them are widely expressed although their appearance and function might be specific to the cell type and developmental stage $^{35,36}$. They are implicated in multiple processes associated with cell cycle, apoptosis, differentiation, regeneration, metabolic adaptation and synaptic plasticity ${ }^{37,38}$. We assume if there is a dysregulated microRNome background of the maladaptive stress reaction in the MD brain, and this microRNA signature might be detectable in other cells of the body.

Compared with other types of RNAs, microRNAs exhibit higher stability even under harsh conditions, i.e. long storage at room temperature ${ }^{39}$ and in extreme $\mathrm{pHs}^{40}$. Postmortem and animal studies revealed microRNA alterations in the brain, blood and cerebrospinal fluid $(\mathrm{CSF})$ in $\mathrm{MD}^{41}$, anxiety ${ }^{42}$, bipolar disorder ${ }^{43}$, and schizophrenia ${ }^{44,45}$. Furthermore, microRNAs are involved in the gene expression and behavior modulatory effects of stress, psychotropic and antidepressant drugs ${ }^{46,47}$. These suggest that microRNAs can be used to assess and monitor the body's pathophysiological state ${ }^{48}$. However, the results are controversial and the association between the brain and peripheral microRNA changes is poorly understood.

\subsection{Study 2 - A PSYChOPHARMACOLOGiCAl ASSAY}

\subsubsection{Human induced pluripotent and neural cells in psychiatric research}

In 2007, Yamanaka and Takahashi showed that human postmitotic somatic cells can be reprogrammed into induced pluripotent stem cells (iPSCs) and further differentiated into all three germlines ${ }^{49}$. Since then, iPSC-research is apparently the most rapidly developing field of bioscience with exceptional possibilities in in vitro disease modeling, regenerative medicine and drug development. Numerous reprogramming protocols have been published differing in the applied factors, delivery method and culturing condition ${ }^{50}$. Summarising, a set of transcriptional factor genes can be transfected into the cells via integrating (lenti- and retroviral) or non-integrating (adenovirus, episomal vector, synthetic mRNA) vectors or direct protein delivering tools ${ }^{51,49,52}$. After initial induction, endogenous NANOG, SOX2 and OCT-4 expression indicate pluripotency of iPSCs which can be maintained for theoretically unlimited time or differentiated into committed cell lines, such as neural progenitors (iNPC) and mature 
neurons (iNC). These provide opportunity to study neuropathophysiology grounded by the patients' individual genotype.

The differentiation of iPSCs is thought to follow in vivo spatio-temporal developmental pathways ${ }^{53,54}$ with highly conserved milestones among embryonic, early postnatal and adult neurogenesis ${ }^{55}$. Remarkably, a combination of growth factors/small molecules is sufficient to evoke high neurotransmitter specificity: GABAergic cortical interneurons ${ }^{56,57}$; dopaminergic midbrain neurons ${ }^{58,59}$; or glutamatergic cortical neurons ${ }^{60}$.

\subsubsection{Modeling hippocampal neurogenesis}

Neurogenesis, defined here by the generation of mature neurons from precursors, continues lifelong in two brain regions: the subventricular zone (olfactory bulb migratory pathway) and the subgranular zone of the hippocampus ${ }^{61}$. In the latter, proliferating radial and non-radial precursors give rise to neuroblasts through intermediate progenitor cells, an estimated number of 500-1000 neurons per day. New, immature neurons migrate into the inner granule cell layer and differentiate into glutamatergic dentate granule cells, grow axons and dendrites and integrate into efferent pathways and the local dentate gyrus - CA3 circuitry (i.e. mossy fibers) ${ }^{62}$.

In 2014, Yu et al. produced hippocampal glutamatergic granule neurons from iPSCs using key developmental cues specific to the dentate gyrus (DG) identity and opened the doors to model adult CNS neurogenesis in vitro ${ }^{63}$. The generated neural cells expressed mature DG neural marker PROX1 and TBR1, established functional synapses and were able to incorporate into mouse DG in vivo.

Adult neurogenesis plays inevitable role in learning and memory ${ }^{64}$. It is a dynamic, fine-tuned process containing several stages such as proliferation, fate determination, selective death/survival and maturation. It is modulated by various intrinsic and extrinsic stimuli from neural, glial and vascular cells or the endocrine system ${ }^{64}$. This complexity and inimitability makes neurogenesis so ambitious subject of in vitro researches. Dysfunctional neurogenic patterns are indicated in psychiatric disorders (recently reviewed by Apple et $\mathrm{al}^{65}$ ). For instance, the neurogenic rate is reduced in stress, MD or schizophrenia which might be reversed by antidepressant and atypical antipsychotic (AAP) medication ${ }^{66,67}$. 


\section{STUDY OBJECTIVES}

We hypothesize that MD is a systemic, maladaptational disorder with a genetic background, thus it can be studied in peripheral cells on a transcriptome level, particularly after stress treatment. We aimed to answer the following questions:

(1) How metabolic stress alters the mRNA and microRNA expression of HDFs (in other words: the manifestation of stress-response in the transcriptome level)?

(2) Are there any differences between the mRNome and microRNome of HDFs of MD and control subjects (i.e. the footprints of diathesis on the transcriptome of peripheral cells)?

(3) Are there any differences in the stress response of MD and control HDFs (the maladaptation-associated mRNA and microRNA-profiles, in effect)?

In Study 2, we aimed to investigate how various antipsychotics (APs) affect the gene expression of differentiating and maturing hippocampal granule cells in vitro. Thus, we set up an in vitro pharmacological assay using neural cells originated from human iPSCs and treating them with typical AP haloperidol (HL) or with atypical olanzapine (OL) and risperidone (RP) at two different concentrations.

\section{MATERIALS AND METHODS}

\subsection{Study 1 - Diathesis-STRESS IN FibRoblasts}

\subsubsection{Skin biopsy and fibroblast cultures}

Skin biopsy was obtained from 16 patients with MD (12 females and 4 males) and 16 healthy control subjects (CNT) matched in age, race and gender (Table 1). The diagnosis of current MD episode was established according to the Structured Clinical Interview for DSMIV-TR (SCID). Exclusion criteria were any other current or past diagnosis on DSM primary axis I or any medical state which could contraindicate the skin biopsy (including bleeding disturbances, lidocaine hypersensitivity, pregnancy or lactation). The punch biopsy was performed on the lateral side of the upper arm as previously described by Manier et al. ${ }^{68}$. The skin was cleaned with iodine and alcohol prep, local anesthesia was induced by $1 \%$ lidocaine injection. The Vanderbilt University Institutional Review Board (IRB, Nashville, TN, USA) approved the study and written informed consent was obtained from all study participants before any procedures were conducted.

The $1 \times 2 \mathrm{~mm}$ skin biopsy sample was transferred in a culture flask containing standard Dulbecco's modified Eagle medium (DMEM; Mediatech) without serum and processed the 
Table 1 Study subjects of Study 1

(BMI: body mass index)

\begin{tabular}{|c|c|c|c|c|c|c|c|c|c|c|c|}
\hline 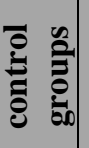 & 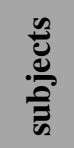 & ס్ర & $\sum_{i=0}^{E}$ & 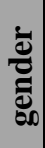 & 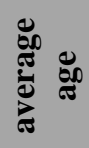 & 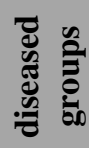 & 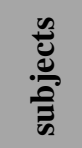 & 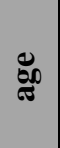 & $\sum_{i \in}^{E}$ & 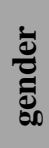 & 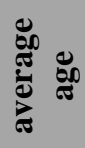 \\
\hline \multirow{4}{*}{ 莺 } & $\mathrm{C} 1$ & 34 & 23 & \multirow{4}{*}{$\begin{array}{l}\frac{0}{\tilde{J}} \\
\frac{\pi}{\tilde{z}}\end{array}$} & \multirow{4}{*}{37.5} & \multirow{4}{*}{$\overline{\mathrm{Z}}$} & D1 & 38 & 32 & \multirow{4}{*}{$\frac{\stackrel{0}{\tilde{J}}}{\stackrel{\Xi}{\Xi}}$} & \multirow{4}{*}{36.5} \\
\hline & $\mathrm{C} 2$ & 38 & 21 & & & & $\mathrm{D} 2$ & 33 & 42 & & \\
\hline & C3 & 48 & & & & & D3 & 46 & 31 & & \\
\hline & $\mathrm{C} 4$ & 30 & & & & & D4 & 29 & 19 & & \\
\hline \multirow{4}{*}{$\sum_{U}^{N}$} & C5 & 35 & 27 & \multirow{4}{*}{ 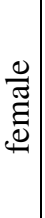 } & \multirow{4}{*}{32.3} & \multirow{4}{*}{$\tilde{\hat{\Sigma}}$} & D5 & 34 & & \multirow{4}{*}{ 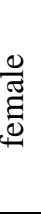 } & \multirow{4}{*}{31} \\
\hline & C6 & 40 & 21 & & & & D6 & 37 & & & \\
\hline & C7 & 27 & 22 & & & & D7 & 27 & 46 & & \\
\hline & $\mathrm{C} 8$ & 27 & 35 & & & & D8 & 26 & 31 & & \\
\hline \multirow{4}{*}{$\sum_{0}^{m}$} & C9 & 51 & 26 & \multirow{4}{*}{ } & \multirow{4}{*}{49.0} & \multirow{4}{*}{$\stackrel{\text { }}{\stackrel{2}{z}}$} & D9 & 51 & 29 & \multirow{4}{*}{ } & \multirow{4}{*}{49.8} \\
\hline & $\mathrm{C} 10$ & 49 & 26 & & & & D10 & 52 & 24 & & \\
\hline & $\mathrm{C} 11$ & 52 & 26 & & & & D11 & 53 & 28 & & \\
\hline & C12 & 44 & 25 & & & & D12 & 43 & 51 & & \\
\hline \multirow{4}{*}{$\underset{⿱ 乛}{\mathbb{Z}}$} & $\mathrm{C} 13$ & 20 & 24 & \multirow{4}{*}{ ָू } & \multirow{4}{*}{22.3} & \multirow{4}{*}{ 苚 } & D13 & 22 & 50 & \multirow{4}{*}{ 袍 } & \multirow{4}{*}{22.5} \\
\hline & $\mathrm{C} 14$ & 22 & 27 & & & & D14 & 22 & 23 & & \\
\hline & $\mathrm{C} 15$ & 22 & 22 & & & & D15 & 23 & 30 & & \\
\hline & C16 & 25 & 31 & & & & D16 & 23 & 18 & & \\
\hline
\end{tabular}

same day. The biopsy was cut into pieces and incubated in trypsin/ collegenase mix at $37^{\circ} \mathrm{C}$ for 1 hour. Standard DMEM $(25 \mathrm{mM}$ glucose, $1 \mathrm{mM}$ sodium pyruvate) supplemented with $2 \mathrm{mM}$ Lglutamine (Mediatech), 10\% fetal bovine serum (FBS; Thermo Scientific) and penicillin/ streptomycin (MediaTech) was added and the cells were pelletted. They were resuspended in standard medium and placed in $60 \mathrm{~mm}$ tissue culture plates.

All fibroblasts were cultured with standard medium at $37^{\circ} \mathrm{C}$ in a

humidified atmosphere containing $5 \% \mathrm{CO}_{2}{ }^{105}$. The medium was changed 3 times per week. Cells were cultured simultaneously, inspected daily by microscopy and subcultured using $0.05 \%$ trypsin/0.02\% EDTA solution (Invitrogen) ${ }^{69}$. The samples were subcultured for 5-10 passages to minimize in vivo effects. After 2-3 weeks, between passages 5-10 the cultures reached confluency, cells were trypsinized, collected and counted.

\subsubsection{Metabolic stress treatment}

Three plates were initiated from each single cell line $\left(1.2 \times 10^{6}\right.$ cells per plate). After overnight adherence, the medium was changed with two different metabolic stress conditions: (1) glucose deprived, galactose-containing (GAL) medium: DMEM deprived of glucose (Mediatech), supplemented with $10 \mathrm{mM}$ galactose (Sigma-Aldrich); (2) reduced lipid (RL) medium: DMEM containing $25 \mathrm{mM}$ glucose, supplemented with lipid-reduced FBS (Thermo Scientific); and (3) standard (STD) medium: DMEM with $25 \mathrm{mM}$ glucose (Figure 1).

All cultures were grown for seven days; the medium was changed 3 times per week. Cell growth and proliferation were not affected by GAL or RL treatment. After one week the cells were washed with ice-cold phosphate-buffered saline (PBS) twice, trypsinized, pelleted (700 $\mathrm{g}$ for $8 \mathrm{~min}$ on $18^{\circ} \mathrm{C}$ ) and stored at $-80^{\circ} \mathrm{C}$ until RNA isolation. 


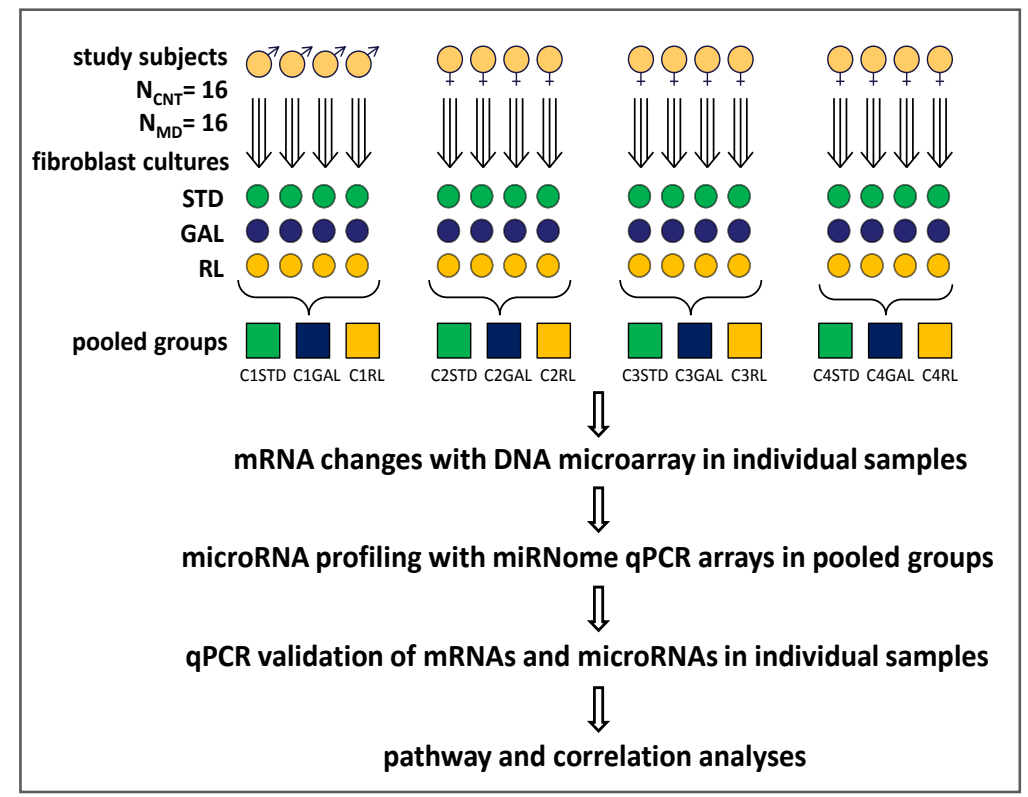

Figure 1. Experimental plan of Study 1

\subsubsection{RNA isolation}

Total cell RNA and small RNA fractions were isolated from the frozen samples using the mirVana miRNA isolation Kit (Ambion) according to manufacturer's instructions. In brief, the cell pellet was suspended in $600 \mathrm{ml}$ Lysis/Binding Solution, then 60 $\mathrm{ml}$ Homogenate Additive was added. After 10 minutes

incubation 1.25 volume of $100 \%$ ethanol was added to the aqueous phase for total RNA isolation. The supernatant was purified using the proprietary solutions provided by the manufacturer. For small RNA isolation, we added 0.33 volume of $100 \%$ ethanol to the aqueous phase and two-third volume of $100 \%$ ethanol to the flow-through. The total RNA and small RNA species were collected with $100 \mathrm{ml}$ Elution Solution and quantified by NanoDrop ND-2100 (Thermo Scientific). Agilent 2100 Bioanalyzer was used to determine the quality and size distribution of the RNA. All samples showed an RNA Intergity Number (RIN) > 8.0. The samples were stored at $-80^{\circ} \mathrm{C}$ until used.

\subsection{4 mRA expression assessment with DNA microarray}

Complementary DNA (cDNA) was generated using $2 \mu \mathrm{g}$ of total RNA. cDNA synthesis, amplification, and labeling were performed using the Enzo Life Sciences SingleRound RNA Amplification and Biotin Labeling System. Biotin labeled, fragmented, amplified RNA (5 $\mu \mathrm{g}$ ) was hybridized to an Affymetrix GeneChip HT HG-U133_PM Array Plate at the Vanderbilt Microarray Shared Resource facility. Segmented images from each microarray were normalized and $\log 2$ transformed using GC-robust multi-array analysis ${ }^{70}$.

After standard image segmentation of the microarray images, RMA normalization was performed using GenePattern software. Average expression values for each group (MD or CNT samples; GAL, RL or STD conditions) were calculated for each gene probe. The magnitude of expression change was determined by the average logarithmic ratio (ALR) using the below described formulas. 
1. To measure the stress induced gene expression changes in CNT or MD fibroblasts: $\mathrm{ALR}=\operatorname{mean}\left(\mathrm{CNT}_{\mathrm{GAL}}\right)-\operatorname{mean}\left(\mathrm{CNT}_{\mathrm{STD}}\right)$ or $\mathrm{ALR}=\operatorname{mean}\left(\mathrm{CNT}_{\mathrm{GAL}}\right)-\operatorname{mean}\left(\mathrm{CNT}_{\mathrm{STD}}\right)$.

2. To analyze the transcriptome differences between the MD and CNT samples:

$\mathrm{ALR}=\operatorname{mean}\left(\mathrm{MD}_{\mathrm{STD}}\right)-\operatorname{mean}\left(\mathrm{CNT}_{\mathrm{STD}}\right)$.

Student's paired and grouped two-tailed $p$-values were used as indicators for significant change. A gene was considered to be differentially expressed when it met the dual criteria of ALR $>0.585(50 \%)$ and both pairwise $p$-value and groupwise $p$-value $\leq 0.05$.

\subsection{1 mRNA profiling data validation by quantitative polymerase chain reaction}

In order to validate our mRNA data, we measured relative mRNA levels of selected genes by quantitative polymerase chain reaction (qPCR). First, based on our pathway analyses we selected a panel of 10 genes involved in lipid biosynthesis in the CNT GAL-, RLtreated samples $(n=16-16)$ : stearoyl-CoA desaturase (SCD); fatty acid binding protein 3 (FABP3); 7-dehydrocholesterol reductase (DHCR7); insulin induced gene 1 (INSIG1); quinolinate phosphoribosyltransferase (QPRT); 3-hydroxy-3-methylglutaryl-CoA reductase (HMGCR); 3-hydroxy-3-methylglutaryl-CoA synthase 1 (HMGCS1); low density lipoprotein receptor (LDLR); fatty acid desaturase 1 and 2 (FADS1, FADS2) and 2 housekeeping genes: actin beta (ACTB) and glyceraldehyde-3-phosphate dehydrogenase (GAPDH). cDNA was synthesized from total RNA (500 ng) using RT2 First Strand Kit (Qiagen) according to the manufacturer's instructions. The 96-well custom-designed RT2 Profiler PCR Arrays (Qiagen) were assayed on the real-time cycler ABI Prism 7300 System (Applied Biosystems). The exported Ct values were analyzed using SABiosciences PCR Array Data Analysis Template Excel and compared using a paired experimental design.

For validation of the mRNA data from the STD-treated MD and CNT samples we measured 13 genes by qPCR: heparin-binding EGF-like growth factor (HBEGF); major histocompatibility complex, class II invariant chain (CD74); glutathione S-transferase theta 1 (GSTT1); major histocompatibility complex class II DR alpha; DQ beta 1; DP beta 1 ; DQ alpha 1; DP alpha 1 (HLA-DRA; -DQB1; -DPB1; -DQA1, -DPA1); interleukin 11 (IL11); Met proto-oncogene (MET); protocadherin 10 (PCDH10); S100 calcium binding protein B (S100B); tumor necrosis factor receptor superfamily, member 19 (TNF19), and a housekeeping gene (glyceraldehyde-3-phosphate dehydrogenase (GAPDH)). cDNA was generated with random primers using High Capacity cDNA Reverse Transcription Kit (Applied Biosystems). Primers with efficiency $>85 \%$ were used in SYBR Green based PCRs 
(Life Technologies). Each sample was tested in four technical replicates on an ABI Prism 7300 thermal cycler (Applied Biosystems). The $\mathrm{Ct}_{(\mathrm{GAPDH})}$ was used for normalization.

\subsection{2 microRNA expression assessment with PCR}

From small RNA samples cDNA was prepared with miScript II RT Kit (Qiagen) using miScript HiSpec Buffer. The DNA was quantified with Nano Drop ND-1000 spectophotometer, yielding 693-839 ng/ml. After reverse transcription, the individual sample cDNAs were pooled into four groups in equal proportions based on the gender and age. Each group contained material from 4 individuals (Table 1). The relative amounts of 1008 microRNAs were measured with Human miRNome miRNA PCR Arrays using miScript SYBR Green PCR Kit (Qiagen) according to the manufacturer's instructions. For microRNA expression quantification comparative $\mathrm{Ct}$ method was used with SNORD61, SNORD68, SNORD72, SNORD95, SNORD96A and RNU6-2 as housekeeping genes. A microRNA was considered differentially expressed if it showed a $|\Delta \Delta \mathrm{Ct}| \geq 0.3785$ (at least $30 \%$ difference between the average $\Delta \mathrm{Ct}$ values) and $p$-value of $\leq 0.05$.

\subsection{3 microRNA data validation by individual PCR}

Twenty-two microRNAs were selected for follow up on the 16 individual, not-pooled samples (hsa-miR-146b-5p, hsa-miR-550a, hsa-miR-129-3p, hsa- miR-214, hsa-miR-21, hsamiR-22, hsa-miR-132, hsa-miR-376c, hsa-miR-19a, hsa-miR-195, hsa-miR-181a, hsa-miR486-5p, hsa- miR-377, hsa-miR-424, hsa-miR-542-3p, hsa-miR-22, hsa-miR-103a, hsa-miR376b, hsa-miR-29b, hsa-miR-185, hsa-miR-564, hsa-miR-34a). The assays were identical to the one used on the pooled samples described above: Human miRNome miRNA PCR Arrays using miScript SYBR Green PCR Kit (Qiagen).

\subsection{4 mRNA and microRNA data analysis}

The differentially expressed genes were subjected to a two-way hierarchical clustering analysis using GenePattern software (Broad Institute). For mRNAs, this was performed using the RMA normalized $\log ^{2}$ gene expression values, while for the microRNAs the $\Delta \mathrm{Ct}$ values were used. Correlations were calculated in MS-Excel 2010 using Pearson coefficient. In addition, gene set enrichment analysis (GSEA) was carried out with the GenePattern software based on the BioCarta defined molecular pathways (San Diego). The calculated normalized enrichment score (NES) reflects the degree to which a gene set is overrepresented in the ranked list of genes. 
To reveal the correlations between the mRNome and microRNome alterations, we searched the miRDB online database (based on miTarget2 dataset) for the target genes of the significantly changed microRNAs ${ }^{71}$. Pathways potentially altered by the significantly changed microRNAs were identified with DIANA-mirPath software. This a priori, in silico computational tool combines Targetscan 5.1 and KEGG pathways. First, it calculates the union of targeted genes of the selected microRNAs, than performs an enrichment analysis. DIANA-mirPath was set to use to identify possible mRNA targets ${ }^{72,73}$. Gene sets were considered significantly differentially expressed at $p \leq 0.05$.

\subsection{StUdy 2 - A PSYChOPHARMACOLOGICAL ASSAY}

\subsection{1 iPSC and NPC cultures}

Human iPSCs were differentiated into Prox 1 positive hippocampal granule neurons according to the protocol published by $\mathrm{Yu}$ et al. $(2014)^{63}$. All the cell cultures were grown under standard conditions $\left(37^{\circ} \mathrm{C}, 5 \% \mathrm{CO}_{2}\right)$ on 6-well-plates except those for further immunocytochemistry, which were loaded into confocal imaging chambers. All media were supplemented with combined antibiotic-antimycotic (Life Tech.).

hiPSCs (line 6/2/F, provided by the Laboratory of Genetics, Salk Institute for Biological Studies, CA USA) were cultured feeder-free with mTeSR media (Stemcell Tech.) on matrigel (BD Biosciences) coated plates. The media was changed daily and the clumps were subcultured via mechanical dissociation after collagenase pre-digestion.

- Day 1: after mechanical dissociation, whole colonies were transferred onto ultra-low adherence dishes for free-floating embryoid body (EB) formation. Differentiating media contained N2B27 media (DMEM/F12-Glutamax plus $\mathrm{N} 2$ and B27; Invitrogen), anticaudalizing factors DKK1 $(0.5 \mu \mathrm{g} / \mathrm{ml}$; PeproTech), Noggin $(0.5 \mu \mathrm{g} / \mathrm{ml}$; Life Tech.), SB431542 (10 $\mu \mathrm{M}$; Sigma) and Cyclopamine ( $1 \mu \mathrm{M}$; Merck). Medium was changed twice a week.

- Day 20: EBs were plated onto poly-L-ornithine and laminin (PORN/L; Sigma) coated plates in N2B27 media supplemented with laminin ( $1 \mu \mathrm{g} / \mathrm{ml}$, Life Tech.). Medium was changed three times per week.

- Day 27-30: adhered neural rosette-containing EBs were manually collected, washed twice with PBS and dissociated with accutase (Life Tech.). Cells were plated onto PORN/L dishes with NPC media (N2B27 media plus bFGF2 $(20 \mathrm{ng} / \mathrm{ml})$ and laminin $(1 \mu \mathrm{g} / \mathrm{ml}))$ changed 3 times/week. NPCs were subcultured using accutase after reaching confluency about 70-90\%. 
- After gaining enough experimental material, P2 NPCs were subcultured onto PORN/L 6-well plates $\left(1.7 \times 10^{5}\right.$ cells/well; 3 wells/treatment) or confocal imaging chambers. On the following day, NPC medium was replaced with differentiating medium containing APs.

\subsubsection{Neural differentiation and treatment with antipsychotics}

We aimed to differentiate NPCs into hippocampal granule neurons and treat the differentiating cells simultaneously with APs. In details, NPC proliferation was arrested by bFGF2 withdrawal and hippocampal granule cell differentiation was provoked by N2B27 media supplemented with ascorbic acid (200 nM, Sigma), dibutiryl-cAMP (500 mg/ml, Sigma), laminin (1mg/ml), BDNF (20 ng/ml, R\&D), Wnt3a (20 ng/ml, R\&D), and 1\% fetal bovine serum (FBS, Fisher Scientific).

Table 2. Treatment groups in Study 2

Haloperidol (HL), olanzapine (OL), and risperidone (RP) were solved in dimethyl sulfoxide (DMSO) and added to the differentiating medium (Table 2). The control cells were treated with the same differentiating medium containing the equal amount of DMSO. The culturing media was changed 3 times per week for 19 days.

\begin{tabular}{|c|c|c|c|}
\hline group & treatment & \multicolumn{2}{|c|}{ concentration } \\
\hline $\mathbf{H L}_{\text {low }}$ & \multirow{2}{*}{ haloperidol } & $10 \mathrm{ng} / \mathrm{ml}$ & $0,003 \mu \mathrm{M}$ \\
\cline { 3 - 4 } & & $100 \mathrm{ng} / \mathrm{ml}$ & $0,03 \mu \mathrm{M}$ \\
\hline $\mathbf{H L}_{\text {high }}$ & & $50 \mathrm{ng} / \mathrm{ml}$ & $0,16 \mu \mathrm{M}$ \\
\hline $\mathbf{O L}_{\text {low }}$ & \multirow{2}{*}{ olanzapine } & $500 \mathrm{ng} / \mathrm{ml}$ & $1,6 \mu \mathrm{M}$ \\
\hline $\mathbf{O L}_{\text {high }}$ & & $100 \mathrm{ng} / \mathrm{ml}$ & $0,24 \mu \mathrm{M}$ \\
\hline $\mathbf{R P}_{\text {low }}$ & \multirow{2}{*}{ risperidone } & $1000 \mathrm{ng} / \mathrm{ml}$ & $2,4 \mu \mathrm{M}$ \\
\hline $\mathbf{R P}_{\text {high }}$ & & \multicolumn{2}{|c|}{$0,2 \mu \mathrm{l} / \mathrm{ml}$} \\
\hline $\mathbf{C N T}_{n n y}$ & DMSO & \multicolumn{2}{|c|}{} \\
\hline
\end{tabular}

Notably, we found only 4 previous studies which applied APs on human, postmitotic neural cultures. HL was used at concentrations of $0.1-5.0 \mu \mathrm{M}$ for 5 days $^{74} ; 1 \mu \mathrm{M}$ (4 days) ${ }^{75}$; or $3 \mu \mathrm{M}$ (minutes) ${ }^{76}$. RP was administered at $0.1-10 \mu \mathrm{M}$ for 5 days $^{74}$, while OL was used at 1$3 \mu \mathrm{M}$ for 48 hours $^{77}$. Because we planed to apply these drugs for 19 days and HL reported to have neurotoxic effects in higher concentrations, we were especially cautious about the dosages. For instance, Post et al. found that HL reduced cell viability by $\sim 20 \%$ at a concentration of $1 \mu \mathrm{M}$ among mouse hippocampal neurons after $20 \mathrm{~min}^{78}$.

\subsubsection{Cell characterization}

To investigate the pluripotency hallmarks of $6 / 2 / \mathrm{F}$ hiPSCs, we used fluorescence activated cell sorting (FACS) ${ }^{79}$. Single cell suspensions were prepared by collagenase predigestion and mechanical dissociation. Cells were washed with PBS containing 0,5\% BSA and incubated with directly labeled anti-human SSEA4-PE mouse monoclonal antibody at $37^{\circ} \mathrm{C}$ for $30 \mathrm{~min}$. Dead cells were labeled with Zombie. For cell sorting, we used an Attune $^{\mathrm{TM}}$ NxT flow cytometer. 
For immunostaining iPSC and

Table 3. Antibodies for cell typisation

differentiated neural cultures were washed gently twice with DPBS and fixed in $4 \%$ PFA at room temperature for $30 \mathrm{~min}$. The samples were rinsed in DPBS 3 x $5 \mathrm{~min}$ and blocked with DPBS++ (3\% normal serum in DPBS with $0,1 \%$ Triton-X) for 30-60 min. After overnight incubation with

\begin{tabular}{|l|l|c|}
\hline antibody & \multicolumn{1}{|c|}{ company } & dilution \\
\hline \multicolumn{2}{|l|}{ stem cell markers } \\
\hline NANOG (goat polyclonal) & R\&D & $1: 50$ \\
\hline OCT3/4 (mouse monoclonal) & Thermo Fisher & $1: 50$ \\
\hline neural markers & \multicolumn{2}{|l}{} \\
\hline PROX-1 (goat polyclonal) & R\&D & $1: 100$ \\
\hline MAP2 (mouse monoclonal) & Sigma & $1: 500$ \\
\hline
\end{tabular}
primary antibodies (Table 3) at $4^{\circ} \mathrm{C}$, samples were washed with DPBS twice and blocked in DPBS++ for 30-60 min. The secondary antibodies (Alexa Fluor conjugated anti-mouse, antigoat or anti-rabbit antibodies) were diluted with DPBS++ and incubated at room temperature for 2-3 hours.

\subsubsection{RNA isolation, reverse transcription}

After 19 days of differentiation and psychopharmacon-treatment, total cell RNA was isolated with Trizol (Invitrogen) following the manufacturer's protocol. Briefly, cells were washed in ice cold PBS and lysate directly in $1 \mathrm{ml}$ of ice cold Trizol Reagent. The samples were stored at $-80^{\circ} \mathrm{C}$ until usage. For RNA isolation, $200 \mu \mathrm{l}$ chloroform was added to the unmelted samples, vortexed and incubated at room temperature for 2-3 minutes. After centrifuging $\left(12000 \mathrm{~g}, 4^{\circ} \mathrm{C}, 15 \mathrm{mins}\right)$ the upper aqueous phase was transfered and diluted with $500 \mu \mathrm{l}$ isopropyl alcohol and $1 \mu 1$ glycogen sol. $(1 \mathrm{mg} / \mathrm{ml})$. Following 10 minutes incubation at RT, samples were spinned down $\left(12000 \mathrm{~g}, 4^{\circ} \mathrm{C}, 15 \mathrm{mins}\right)$ and the supernatant was removed. The pellet was washed with $1 \mathrm{ml} 75 \%$ ethyl alcohol, centrifuged $\left(12000 \mathrm{~g}, 4^{\circ} \mathrm{C}, 15 \mathrm{mins}\right)$, airdryed and resuspended into $20 \mu \mathrm{l}$ RNAse free water. The quality of the RNA samples were assessed with Nanodrop 2000 Spectrophotometer (Thermo Scientific).

Complementary DNA (cDNA) synthesis was carried out using the Promega Reverse Transcription System. $1 \mu \mathrm{g}$ total cell RNA in $9.5 \mu 1$ RNase free water was incubated at $70^{\circ} \mathrm{C}$ for 10 minutes than chilled on ice. The reaction mix contained $4 \mu \mathrm{MgCl}_{2}, 1 \mu \mathrm{L}$ of random hexamer primers $(0.5 \mathrm{mg} / \mathrm{ml}), 2 \mu \mathrm{L} 10 \mathrm{x}$ reaction buffer, $1 \mu \mathrm{L}$ recombinant RNasin RNase Inhibitor $(20 \mathrm{U} / \mu \mathrm{L}), 2 \mu \mathrm{L} 10 \mathrm{mM}$ dNTP Mix, $1 \mu \mathrm{L}$ AMV Reverse Transcriptase $(15 \mathrm{U} / \mu \mathrm{L})$. The incubation lasted $10 \mathrm{~min}$ at RT, followed by incubation for $60 \mathrm{~min}$ at $42^{\circ} \mathrm{C}$ and termination of the reaction $\left(90^{\circ} \mathrm{C}, 5 \mathrm{~min}\right)$. Samples were stored at $-20^{\circ} \mathrm{C}$ until further usage. 


\subsubsection{Real-time quantitative PCR and data analysis}

For real-time quantitative polymerase chain reaction (rt-qPCR), we used the following TaqMan PCR probes to analyze the gene expression changes (Thermo Fisher): metabotropic glutamate receptor 2 (mGluR2 or GRM2, Hs00968358_m1); metabotropic glutamate receptor 7 (mGluR7 or GRM7, Hs00356067_m1); vesicular glutamate transporter 1 (VGLUT1 or SLC17A7, Hs00220404_m1); microtubule-associated protein 2 (MAP2, Hs00258900_m1); neuronal differentiation 1 (NeuroD1 or BETA2, Hs01922995_s1); glial fibrillary acidic protein (GFAP, Hs00909233_m1). We chose large ribosomal protein P0 (RPLP0, Hs99999902_m1) for endogenous control to normalize the variations in cDNA quantities from different samples recommended by ${ }^{80,81}$. Each reaction was performed in triplicate for every well ( 9 reactions per gene per treatment group).

The reactions were performed according to the manufacturer's instructions: $5 \mu \mathrm{L}$ template cDNA was used in a final PCR reaction volume of $20 \mu \mathrm{L}$, containing $10 \mu \mathrm{L}$ TaqMan Master Mix, $1 \mu \mathrm{L}$ of 20x forward and reverse primer and probe and $4 \mu 1$ bidestillated water. The conditions for the PCR included 2 min denaturation at $94^{\circ} \mathrm{C}$ followed by 40 cycles of PCR $\left(10 \mathrm{~min}\right.$ at $94^{\circ} \mathrm{C}, 15 \mathrm{~s}$ at $52^{\circ} \mathrm{C}, 60 \mathrm{~s}$ at $\left.60^{\circ} \mathrm{C}\right) . \mathrm{mRNA}$ changes were measured in the StepOnePlus real-time PCR System (Applied Biosystem) using the StepOne v2.1 program.

$\mathrm{Ct}$ was determined manually from the amplification plots. We calculated the normalized gene expression level with the $\Delta \Delta \mathrm{Ct}$ method: average $\Delta \mathrm{Ct}$ (treated) - average $\Delta \mathrm{Ct}$ (control). Statistical significance was tested with unpaired two-sample $t$-test. A gene was considered differentially expressed if $|\Delta \Delta \mathrm{Ct}| \geq 0.3785$ ( $\geq 30 \%$ difference) and $p$-value $\leq 0.05$.

\section{RESULTS}

\subsection{Study 1 - DiatheSiS-STRESS IN FibRoblastS}

\subsubsection{How fibroblasts adapt on transcriptome-level?}

In order to examine the cellular stress response in peripheral tissues we measured the mRNA and microRNA levels after 1 week metabolic stress treatment in fibroblast cultures obtained from healthy, CNT subjects.

GAL-treatment, a mitochondrial and oxidative stress resource affected the expression of 2063 genes compared to STD condition. 1117 genes were overexpressed (54.1\%). Many of these are known to be involved in cell cycle, apoptosis, inflammation, mRNA metabolism and metabolic adaptation. 
The lipid reduced, cholesterol deprived (RL) treatment also resulted in mRNome changes: 984 gene products were significantly altered, 640 of these were increased (65.0\%) (Figure 2).

BioCarta pathway analysis revealed enrichment of 19 gene sets in the GAL-treatment and 15 in the RLtreatment (3 of them repressed). Notably, 4 pathways were found to be overexpressed in both stress conditions

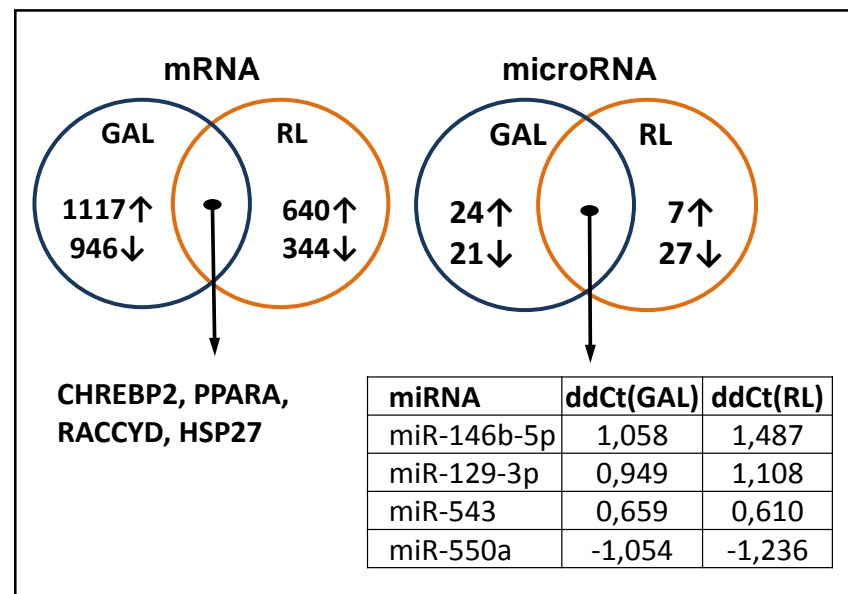

Figure 2. Metabolic stress-induced transcriptome changes in HDFs derived from CNTs.

(Table 4A and B).

Table 4A. and B. Metabolic stress affected pathways in HDFs derived from control subjects. ${ }^{*}$ Commonly upregulated in GAL and RL. 4C. Pathways regulated by the overlapping 4 metabolic stress-induced microRNAs. (NES: normalised enrichment score)

\begin{tabular}{|c|c|c|c|c|c|c|c|}
\hline \multicolumn{3}{|c|}{ A. GAL-affected } & \multicolumn{3}{|l|}{ B. RL-affected } & \multicolumn{2}{|c|}{ C. microRNA-modulated } \\
\hline pathways & NES & $p$-value & pathways & NES & $p$-value & KEGG pathways & $p$-value \\
\hline RACCYCD* & 1.93 & 0.000 & PPARA* & 1.80 & 0.000 & ErbB signaling & 0.000 \\
\hline CELLCYCL & 1.83 & 0.000 & P38MAPK & 1.56 & 0.010 & Regulat. of actin cytosk. & 0.000 \\
\hline SRCRPTP & 1.70 & 0.000 & VIP & 1.60 & 0.010 & Dopaminergic synapse & 0.001 \\
\hline P53 & 1.70 & 0.002 & BAD & 1.48 & 0.016 & Osteoclast differentiation & 0.001 \\
\hline TEL & 1.73 & 0.011 & CHREBP2* & 1.64 & 0.023 & Chagas disease & 0.001 \\
\hline STATHMIN & 1.57 & 0.012 & RACCYCD* & 1.65 & 0.028 & Axon guidance & 0.002 \\
\hline CFTR & 1.65 & 0.012 & GCR & 1.46 & 0.038 & Insulin signaling & 0.002 \\
\hline CERAMIDE & 1.67 & 0.012 & HSP27* & 1.49 & 0.041 & MAPK signaling & 0.004 \\
\hline ATRBRCA & 1.60 & 0.016 & $\mathrm{CD} 40$ & 1.58 & 0.044 & FcyR-mediated phagocyt & 0.004 \\
\hline G2 & 1.59 & 0.026 & LYM & 1.37 & 0.048 & FceRI signaling & 0.004 \\
\hline HSP27* & 1.47 & 0.027 & TNFR2 & 1.53 & 0.049 & GnRH signaling & 0.004 \\
\hline CARM_ER & 1.61 & 0.027 & TALL1 & 1.52 & 0.049 & Protein processing in ER & 0.006 \\
\hline PLCE & 1.47 & 0.031 & IL22BP & -1.56 & 0.021 & Wnt signaling & 0.006 \\
\hline CHREBP2* & 1.61 & 0.031 & DNAFRAGM. & -1.51 & 0.030 & Lysine degradation & 0.008 \\
\hline AKAPCENT & 1.53 & 0.032 & AHSP & -1.59 & 0.043 & Circadian rhythm & 0.009 \\
\hline CHEMICAL & 1.56 & 0.033 & & & & Hepatitis B & 0.015 \\
\hline $\mathrm{RB}$ & 1.53 & 0.034 & & & & Chemokine signaling & 0.026 \\
\hline ATM & 1.58 & 0.045 & & & & TLR signaling & 0.037 \\
\hline PPARA* & 1.43 & 0.036 & & & & Melanogenesis & 0.043 \\
\hline & & & & & & $\begin{array}{l}\text { Cytokine-cytokine R } \\
\text { interaction }\end{array}$ & 0.046 \\
\hline & & & & & & Neurotrophin signaling & 0.000 \\
\hline & & & & & & Adherence junction & 0.000 \\
\hline
\end{tabular}


These pathways might be essential for the physiological adaptation independently from the type of stressor: (1) Peroxisome proliferator-activated receptor alpha (PPARA) is a lipid activated pathway which regulates several genes involved in metabolism, energy homeostasis, oxidative stress response, and anti-inflammation. PPARA agonists proved to have protective features in neurodegenerative disorders ${ }^{82,83}$. (2) Carbohydrate-responsive element-binding protein-2 (CHREBP2) pathway. CHREBP is a glucose-sensing, lipogenic transcription factor enabling the cell to adopt to extracellular glucose concentrations. Loss of function mutation results in fatty acid synthesis deficit, and impaired insulin homeostasis ${ }^{84}$. (3) Influence of Ras and Rho proteins on G1 to S transition (RACCYCD) pathway which describes the induction of cyclin D1 expression and cell cycle progression ${ }^{85}$. (4) Stress induction of heat shock protein 27 (HSP27) regulation. HSP27, a class I small-HSP has a chaperon-like activity; it acts as a cytoskeleton stabilizer, anti-oxidant, anti-apoptotic agent, and cell cycle regulator ${ }^{86}$.

Interestingly, both GAL and RL-treatment modified the expression of a set of genes associated with lipid biosynthesis. We validated our microarray data with 10 from these. According to the qPCR data 9 of the 10 genes were significantly altered by both GAL and RL-conditions (HMGCR mRNA levels showed the same direction but did not reach statistical significance). The qPCR and microarray findings showed high correlation $\left(r_{(\mathrm{GAL})}=0.92\right.$; $\left.\mathrm{r}_{(\mathrm{RL})}=0.72\right)($ Table 5).

GAL-treatment modulated 45 microRNAs and RL-condition changed the expression of 34 microRNAs compared to STD culturing. The overlap between the two different metabolic stresses were 4 microRNAs: hsa-miR-146b-5p, hsa-miR-129-3p, hsa-miR-543 were elevated, while miR-550a was downregulated (Figure 2). DIANA mirPath pathway analysis suggested that these 4 microRNAs participate in the regulation of 57 KEGG pathways. Thirty-nine percent of the pathways (22) are controlled by all 4 microRNAs, and 16 of these are intracellular signaling pathways (Table 4C).

Although, the list of the GAL and RL-treatment affected microRNAs differed from each other, when we correlated the GAL-modified microRNAs with their expression in the RL-treatment, we found significant correlation $(\mathrm{r}=0.71, p<0.001)$. Similarly, data of RLaffected microRNAs correlated with the levels measured in GAL $(\mathrm{r}=0.65, p<0.005)$. These indicate that the most affected microRNAs were not identical in the different conditions but the metabolic stresses resulted in a similar overall microRNA pattern in the CNT cells. 
Table 5. Validation of microarray data with $q P C R$ (NS: no significant change)

\begin{tabular}{|c|c|c|c|c|}
\hline \multirow[b]{3}{*}{ Gene } & \multicolumn{2}{|c|}{ GAL vs. STD } & \multicolumn{2}{|c|}{ RL vs. STD } \\
\hline & microarray & qPCR & microarray & qPCR \\
\hline & ALR & $-\Delta \Delta \mathbf{C t}$ & ALR & $-\Delta \Delta \mathrm{Ct}$ \\
\hline$S C D$ & 2.02 & 2.66 & 1.82 & 2.23 \\
\hline$F A B P 3$ & 1.86 & 2.80 & 1.89 & 3.10 \\
\hline DHCR7 & 1.18 & 1.42 & 1.82 & 2.49 \\
\hline INSIGI & 1.43 & 1.79 & 1.61 & 2.16 \\
\hline$Q P R T$ & 1.42 & 2.03 & 1.96 & 2.71 \\
\hline$H M G C R$ & 0.70 & 0.43 & 1.50 & $\mathrm{NS}$ \\
\hline HMGCS1 & 1.17 & 1.20 & 1.64 & 2.40 \\
\hline$L D L R$ & 1.08 & 1.46 & 1.55 & 2.35 \\
\hline FADS1 & 1.08 & 1.36 & 1.22 & 1.57 \\
\hline FADS2 & 1.00 & 1.84 & 1.51 & 2.50 \\
\hline correl & \multicolumn{2}{|c|}{$r^{2}=0.84$} & \multicolumn{2}{|c|}{$r^{2}=0.63$} \\
\hline
\end{tabular}

Since microRNome PCR assays were conducted on pooled samples which could bias the results, we measured 22 microRNAs in the individual, not-pooled samples using the same PCR assays to validate our data. Our data suggest, pooling of the samples did not skew artificially the microRNA pattern observed in GAL and RL.

We were also curious how the microRNA changes present on the level of mRNAs. To determine the crosstalk among these transcripts, we listed the potential target genes of the

online database, miR-129-3p, miR146b-5p, miR-550a and miR-543 have 211, 243, 129 and 921 putative target genes, respectively. Notably, $9.3 \%$ of these genes were differentially expressed under stress (140 out of 1504) and several of them showed transcription alterations in both GAL and RL-treatment. This rate meets our expectations since literature suggests that $10 \%$ of the predicted genes reflect to the regulator microRNAs depending on the cell type, developmental stage and environment.

\subsubsection{Manifestation of MD diathesis in fibroblasts}

We compared the mRNA and microRNA profile of fibroblasts from MD patients and CNT subjects in STD culturing conditions. First, we measured the mRNA levels with DNA microarray: 162 genes were differentially expressed in the MD samples. Remarkably, 114 genes were downregulated, which indicate rather loss-of-fuction genetic dysregulation than gene induction in the

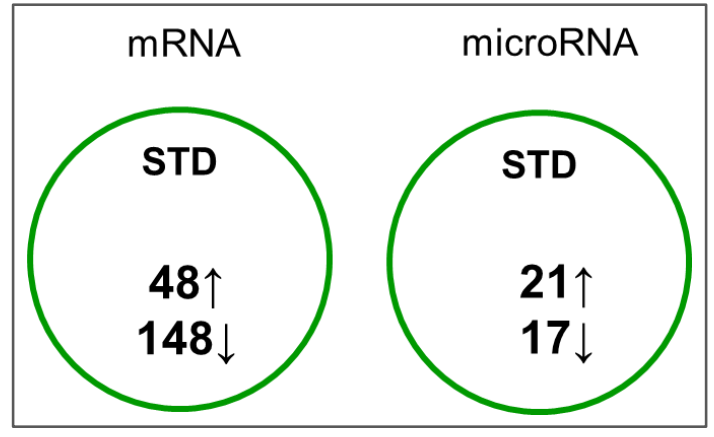

Figure 3. Transcriptome alterations of $M D$ fibroblasts compared to CNT cells in standard conditions

disorder (Figure 3). The most prominent decreases were observed in genes associated with cell-to-cell communication and adhesion, such as protocadherin 10 (PCD10, ALR=1.73); 
tenascin XB (TNXB, ALR=1.30); periplakin $(P P L, \mathrm{ALR}=1.34)$; and hepatocyte growth factor (HGF) receptor $(\mathrm{ALR}=1.21)$.

Table 6. Differentially expressed pathways in MD fibroblasts compared to CNT cells

According to the gene set enrichment

\begin{tabular}{|c|c|c|c|}
\hline Pathway & NES & $p$-value & function \\
\hline Akt signaling & -1.61 & 0.018 & \multirow{3}{*}{ 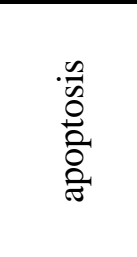 } \\
\hline $\begin{array}{l}\text { TCAPOPTOSIS: HIV } \\
\text { induced T-cell apoptosis }\end{array}$ & 1.53 & 0.036 & \\
\hline $\begin{array}{l}\text { ARF: Arf inhibits } \\
\text { ribosomal biogenesis }\end{array}$ & -1.55 & 0.044 & \\
\hline $\begin{array}{l}\text { CLASSIC: classical } \\
\text { complement }\end{array}$ & -1.49 & 0.026 & \multirow{5}{*}{ 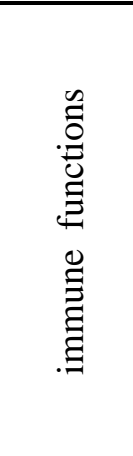 } \\
\hline COMP: complement & -1.42 & 0.047 & \\
\hline $\begin{array}{l}\text { ASBCELL: antigen } \\
\text { dependent B-cell activ. }\end{array}$ & 1.49 & 0.006 & \\
\hline $\begin{array}{l}\text { CSK: activation of Csk by } \\
\text { PKA }\end{array}$ & 1.51 & 0.013 & \\
\hline $\begin{array}{l}\text { TCR activation by Lck } \\
\text { and Fyn tyrosine kinases }\end{array}$ & 1.43 & 0.033 & \\
\hline $\begin{array}{l}\text { CTLA4: co-stimulatory } \\
\text { signal during T-cell activ. }\end{array}$ & 1.44 & 0.037 & \multirow{2}{*}{ 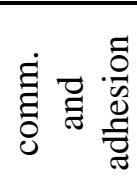 } \\
\hline $\begin{array}{l}\text { NKT: selective expression } \\
\text { of chemokine receptors }\end{array}$ & 1.52 & 0.038 & \\
\hline
\end{tabular}
analysis 6 BioCarta pathways were upregulated and 4 were downregulated. These are known to play role in: apoptosis, cell-to-cell communication and adhesion, and immune functions (Table 6). These mRNA and pathway analysis data concur with peripheral and postmortem brain tissue studies that the genetic background of MD may manifest in immune and (neuro)development related gene set disturbances.

We chose 14 immune-related genes and determined their expression with qPCR to validate our microarray data (genes listed in Materials and Methods). The microarrayreported ALR and qPCR-given $\Delta \Delta \mathrm{Ct}$ values showed high correlation ( $\mathrm{r}=0.84)$, and 12 of the 14 transcripts were significantly changed in the qPCR data also.

The MET/HGF intracellular signaling pathway attracted our attention for its trophic roles. Under normal conditions, the pathway is silent but during development, stress and regeneration, MET tyrosine kinase receptor promotes motility, proliferation, morphogenesis and protects cells against hypoxia and serum deprivation caused dysfunction through the PI3K-Akt and ras-MAPK pathways in neurons ${ }^{87}$ and peripheral cells as well ${ }^{88}$. Paradoxically, HGF (scatter factor) proved to be identical with fibroblast-derived tumor cytotoxic factor (FTCF) and MET activation can provoke apoptosis in tumor cells ${ }^{89}$ and myofibroblasts ${ }^{90}$. This dual, cell-type specific antiapoptotic and proapoptotic property may be essential in regeneration ${ }^{91}$. The pathway participates also in neurodevelopment, and in the prevention of post-stroke neuron death. Serum HGF level was found to correlate with the presence, severity and progression of symptoms in a subgroup of MD patients ${ }^{92}$. Interestingly, Arakaki et al. found that the proapoptotic, antimitotic effect of MET can be suppressed by enhanced GSH production $^{93}$. According to Gibson et al., MD fibroblasts exhibit impaired redox homeostasis and decreased glutathione-regeneration reserve capacity ${ }^{105}$. 
Hence, we decided to compare the expression of genes involved in the MET/HGF pathway in MD and CNT fibroblasts. From the overall 37 pathway-genes 16 were differentially expressed: HGF; phosphoinositide-3-kinase regulatory subunit 1 (PIK3R1); GRB2-associated binding protein 1 (GAB1); son of sevenless homolog 1 (SOS1); Rap guanine nucleotide exchange factor 1 (RAPGEF1); signal transducer and activator of transcription 3 (STAT3); protein tyrosine phosphatase, nonreceptor type 11 (PTPN11); p21/Cdc42/Rac-activated kinase 1 (PAK1); mitogen-activated protein kinase 1 (MAPK1); vcrk sarcoma virus CT10 oncogene homolog-like (CRKL); jun oncogene (JUN); phosphoinositide-3-kinase, catalytic, alpha polypeptide (PTEN); member of RAS oncogene family (RAP1A); mitogen-activated protein kinase kinase 1 (MAP2K1); and v-Ha-ras Harvey rat sarcoma viral oncogene homolog (HRAS).

According to our PCR results, nearly $50 \%$ of the measured microRNAs were expressed in the fibroblast cultures (561 out of 1008). The microRNA profile of MD fibroblasts differed in 38 microRNAs from CNT (more than $30 \%$ change, $p<0.05$ ). 17 were downregulated and 21 showed higher expression levels (Figure 4).

The microRNA levels were determined in pooled groups (4 individuals/group). To exclude any adverse effect of pooling, we measured the expression of hsamiR-21*, hsa-miR-377, hsa-miR193a-3p, hsa-miR-542-3p, hsamiR-22, hsa-miR103a, and hsamiR-185. microRNA expression levels showed high correlation between the pooled and individual

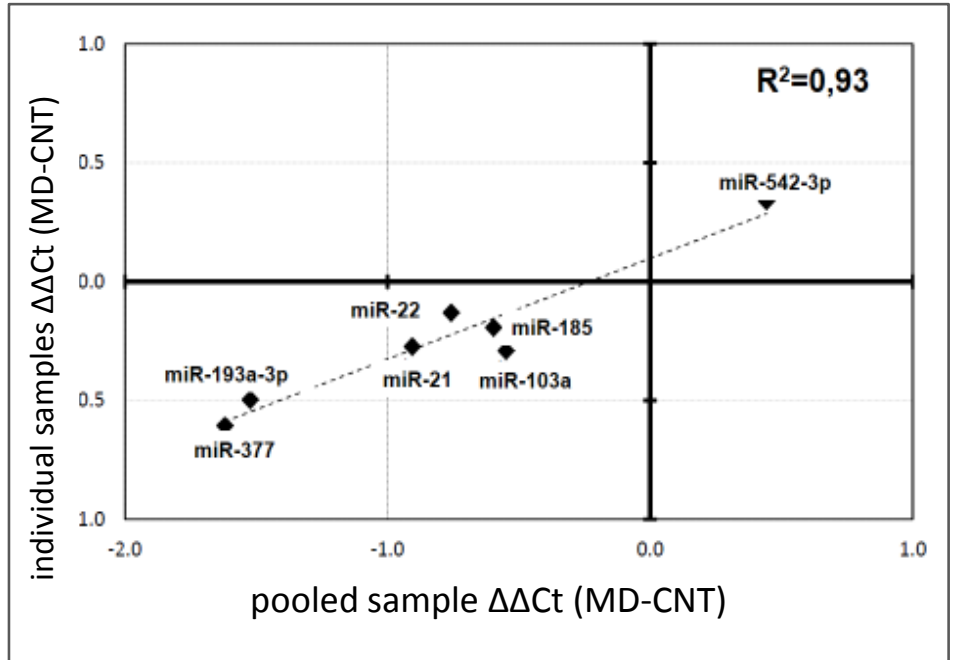

Figure 4. Correlation of microRNA levels in pooled and individual samples sample assessments $\left(\mathrm{R}^{2}=0.93, p<0.001\right)$ (Figure 5). Thus we can assume that grouping and mixing the RNA samples did not reduce the reliability of our PCR array results.

To uncover the possible functional consequences of the MD-specific microRNA profile, we conducted pathway analysis by DIANA mirPath with the 38 differentially expressed microRNAs. According to the results, 19 pathways are controlled by at least $85 \%$ of these microRNAs. Some of them are universal intracellular signaling pathways (e.g. calcium signaling; ubiquitin mediated proteolysis pathway), and others are related to cell cycle (PI3K-Akt signaling; MAPK signaling pathway), cell-to-cell communication and 
adhesion (Wnt; chemokine signaling; focal adhesion pathway), immune functions (Jak-STAT signaling; endocytosis pathway), and neural functions (neurotrophin signaling; long-term potentiation; cholinergic synapse pathway).

We wanted to know if the microRNA alterations could explain the mRNA profile of MD fibroblasts. Thus we searched miRDB for the predicted targets of the 38 microRNAs, and noted if the particular mRNA showed MD-specific expression. Our search revealed that $51 \%$ of the differentially expressed mRNAs were potential target molecules. This rate meets our expectations, since literature suggests that $40-60 \%$ of the human genes are under microRNA regulation. Interestingly, $16 \%$ of the changed microRNAs did not leave fingerprint on the mRNome; and further $10 \%$ had changed in the same direction as the regulated mRNAs. These results suggest that the mRNA signature of MD is appreciably influenced by the microRNome and there is a remarkable

Table 7. Pathways regulated by at least $85 \%$ of the MD-related microRNAs

\begin{tabular}{|lc|}
\hline Pathway & p-value \\
\hline Neurotrophin signaling & $<0.0001$ \\
\hline Pathways in cancer & $<0.0001$ \\
\hline PI3K-Akt signaling & $<0.0001$ \\
\hline MAPK signaling & $<0.0001$ \\
\hline Jak-STAT signaling & 0.008 \\
\hline Ubiquitin mediated proteolysis & $<0.0001$ \\
\hline Endometrial cancer & $<0.0001$ \\
\hline HTLV-I infection & $<0.0001$ \\
\hline Focal adhesion & $<0.0001$ \\
\hline Osteoclast differentiation & $<0.0001$ \\
\hline Hepatitis B & $<0.0001$ \\
\hline Insulin signaling & $<0.0001$ \\
\hline Endocytosis & $<0.0001$ \\
\hline Calcium signaling & $<0.0001$ \\
\hline Non-small cell lung cancer & $<0.0001$ \\
\hline Cholinergic synapse & $<0.0001$ \\
\hline Chemokine signaling & $<0.0001$ \\
\hline Long-term potentiation & $<0.0001$ \\
\hline Wnt signaling & $<0.0001$ \\
\hline
\end{tabular}
interaction on the transcriptomic level. However, considering that $64 \%$ of the differentially expressed mRNAs are targeted by more than one microRNA, and microRNAs can either upor downregulate the same gene depending on the cellular and external environment, we can only establish hypotheses on the exact cause-effect relationships.

\subsubsection{Stress vulnerability of MD-fibroblasts}

We aimed to examine whether genetically determined impaired adaptational potential of MD patients can be demonstrated in stress-exposed peripheral tissues. Hence, we measured the metabolic stress induced transcriptome changes in fibroblasts obtained from MD patients. We found that galactose (GAL) substitution of glucose in the medium resulted in robust transcriptome changes in the MD fibroblasts: 1196 mRNAs were affected, 975 of these upregulated. Comparing the stress response of the MD and CNT cells we found, that $26 \%$ of the GAL-altered genes (310) were MD-specific (Figure 6). Twenty-one pathways participated in the GAL-stress reaction, 9 of them were activated only in the MD samples (Table 8A). 
The RL-treatment had mostly activating effect on the geneexpression pattern in the MD cells (262 upregulated mRNAs from the overall affected 312). The RL induced stress response of MD cells significantly differed from the CNTs: $33.4 \%$ of the mRNA changes were exhibited only in the $\mathrm{MD}$, and not in the CNT

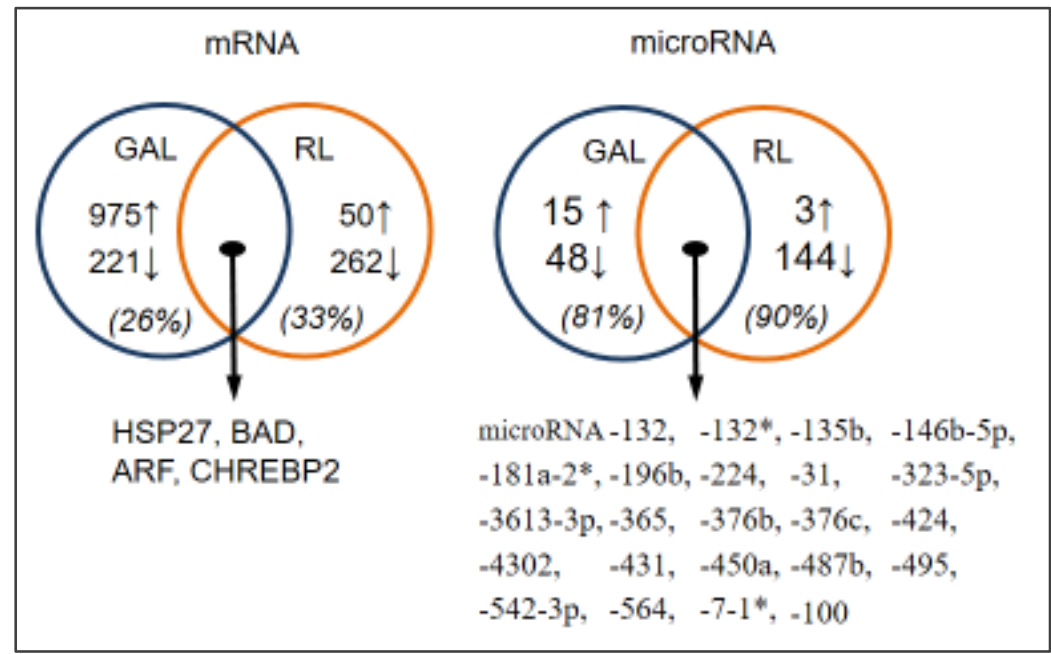

Figure 5. Stress induced transcriptome changes in HDFs from MD patients. Parenthetic percentages show the ratio of $M D$ specific $m R N A$ and microRNA changes. groups (103 genes) (Figure 5). Comparison of pathway analysis data revealed that 10 of the 19 RL-modified gene sets are MD-specific (Table 8 B).

The overlap between the stress induced pathway signatures of MD cells were: HSP27, CHREBP2, BAD and ARF which are known to control apoptosis and cell cycle phase transition. Although, the pathways induced by GAL and RL were different, both condition required cell cycle (e.g. RB, TFF, G2), apoptosis/survival (P53, TRKA, SPRY), migration, immune function (ECM, CDMAC, IL-7, IL-10), metabolism (GLYCOLYSIS) related gene set adaptations.

According to our qPCR array data, 63 microRNAs participated in the GAL-treatment induced stress response of the MD fibroblasts. $81 \%$ of the detected microRNA pattern was MD-specific. The RL-condition resulted in more robust microRNA changes: 147 microRNAs were differentially expressed compared to the STD, $90 \%$ of these were observed only in the MD cells (Figure 5).

Searching the literature for previous data on the GAL and RL-affected microRNAs, we found that $38 \%$ are related to metabolism control, $60 \%$ have role in proliferation and apoptosis, and 30\% participate in cell motility. It is noteworthy, that significant proportion of the changed microRNAs are regulated by tumorsuppressor P53 (GAL: 13\%, RL: 33\%). P53, best known for its role in DNA damage response, is also an important regulator of metabolism, suppress the glycolytic pathway, increases mitochondrial metabolism ${ }^{94}$. Several studies have identified microRNAs as regulators of P53 activity as well as its downstream effectors. For instance, the oncogenic role of miR-122, miR-7 or miR-32 loss has been attributed to the (in)direct regulation of $\mathrm{P} 53^{95,96}$. These and our results might demonstrate that 
the ARF/p53 pathway enhancement may have a strong regulatory effect on the overall gene expression pattern via modulation of certain microRNAs.

The overlap between the GAL and RL-induced miRNome stress response were 23 microRNAs (listed in Figure 5). Six of them were already dysregulated under STD conditions in contrast with CNT samples, which might underpin our hypothesis that the dysregulated microRNA profile in MD contributes to the stress response. Considering the notable differences between the transcriptome of MD and CNT samples, we might assume that the MD cells can reach the required metabolic changes via non-physiological ways which might result in increased vulnerability.

Table 8A. and B. Pathways involved in the stress response of MD fibroblasts. 8C. Pathways might be targeted by $>85 \%$ of those microRNAs which were commonly changed in both GAL and RL-treatment. * MD specific changes. The highlighted pathways were upregulated in both metabolic stresses. (NES: normalized enrichment score)

\begin{tabular}{|c|c|c|c|c|c|c|c|}
\hline \multicolumn{3}{|c|}{ A. GAL-affected } & \multicolumn{3}{|l|}{ B. RL-affected } & \multicolumn{2}{|c|}{ C. microRNA-modulated } \\
\hline Pathway & NES & $p$-value & Pathway & NES & $p$-value & KEGG pathway & $p$-value \\
\hline TEL & 1.98 & 0.000 & RACCYCD & 1.90 & 0.004 & PI3K-Akt signaling* & $<0.0001$ \\
\hline SRCRPTP & 1.73 & 0.002 & TFF* & 1.88 & 0.006 & Regul. of actin cytosk. & $<0.0001$ \\
\hline PTC1* & 1.73 & 0.000 & CD40 & 1.78 & 0.010 & Focal adhesion* & $<0.0001$ \\
\hline $\mathrm{G} 2$ & 1.66 & 0.002 & TRKA* & 1.74 & 0.008 & Neurotrophin signaling & $<0.0001$ \\
\hline CARM_ER & 1.66 & 0.005 & ECM* & 1.67 & 0.023 & Pathways in cancer* & $<0.0001$ \\
\hline HSP27 & 1.65 & 0.011 & PPARA & 1.63 & 0.006 & ErbB signaling & $<0.0001$ \\
\hline $\mathrm{RB}$ & 1.62 & 0.006 & HSP27 & 1.62 & 0.017 & MAPK signaling & $<0.0001$ \\
\hline CERAMIDE & 1.60 & 0.021 & GH* & 1.60 & 0.020 & T cell $\mathbf{R}$ signaling* & $<0.0001$ \\
\hline P53 & 1.58 & 0.022 & GLEEVEC* & 1.58 & 0.036 & Chemokine signaling & $<0.0001$ \\
\hline SET* & 1.58 & 0.032 & ARF* & 1.54 & 0.046 & HTLV-I infection* & $<0.0001$ \\
\hline CHEMICAL & 1.58 & 0.024 & EXTRINSIC* & 1.53 & 0.014 & & \\
\hline ATRBRCA & 1.57 & 0.008 & CHREBP2* & 1.53 & 0.024 & & \\
\hline MPR* & 1.56 & 0.041 & CDMAC* & 1.52 & 0.035 & & \\
\hline CELLCYC. & 1.56 & 0.030 & $\mathrm{BAD}$ & 1.48 & 0.011 & & \\
\hline AKAP95* & 1.56 & 0.046 & SPRY* & 1.45 & 0.041 & & \\
\hline CHREBP2 & 1.56 & 0.022 & FEEDER* & -1.53 & 0.023 & & \\
\hline IL10* & 1.53 & 0.032 & DNAFRAG. & -1.54 & 0.048 & & \\
\hline ARF* & 1.50 & 0.038 & AHSP & -1.56 & 0.035 & & \\
\hline PLCE & 1.47 & 0.030 & GLYCOLYSIS* & -1.63 & 0.004 & & \\
\hline IL7* & 1.46 & 0.025 & & & & & \\
\hline BAD* & 1.43 & 0.013 & & & & & \\
\hline
\end{tabular}


Table 9 Validation of microarray data with $q P C R$.

Our results suggest that the two All results were significant $(p<0,05)$.

different stress conditions required not identical, but very similar gene expression pattern modifications from the CNT cells which phenomena can be explained by the evolutionary conserved, general rules of cellular and systemic adaptation. Whereas GAL and RL-environment resulted in different, stress effect-specific transcriptome changes in the MD cells. RL-medium showed to be especially greater

\begin{tabular}{|c|c|c|c|c|}
\hline \multirow[b]{3}{*}{ Gene } & \multicolumn{2}{|c|}{ GAL vs. STD } & \multicolumn{2}{|c|}{ RL vs. STD } \\
\hline & microarray & qPCR & microarray & qPCR \\
\hline & ALR & $-\Delta \Delta C t$ & ALR & $-\Delta \Delta \mathbf{C t}$ \\
\hline$S C D$ & 2.13 & 2.37 & 1.71 & 2.21 \\
\hline$F A B P 3$ & 1.88 & 3.33 & 1.55 & 3.36 \\
\hline DHCR7 & 1.24 & 1.76 & 1.99 & 2.57 \\
\hline INSIGI & 1.44 & 1.76 & 1.59 & 2.41 \\
\hline$Q P R T$ & 1.67 & 2.07 & 2.02 & 2.65 \\
\hline$H M G C R$ & 0.75 & 0.98 & 1.50 & 1.96 \\
\hline HMGCS1 & 1.21 & 1.46 & 1.73 & 2.30 \\
\hline$L D L R$ & 1.24 & 1.75 & 1.70 & 2.44 \\
\hline FADS1 & 1.08 & 1.36 & 1.23 & 1.58 \\
\hline FADS2 & 0.76 & 1.78 & 1.27 & 2.34 \\
\hline correl & \multicolumn{2}{|c|}{$r=0.78$} & \multicolumn{2}{|c|}{$r=0.43$} \\
\hline
\end{tabular}
challenge. Understanding why these metabolic stresses represent altering challenges in MD may help to get closer to the genetic background of maladaptation.

To validate the microarray-detected mRNA changes, custom qPCR arrays were used to detect the mRNA expression level of 10 stress-affected genes involved in lipid metabolism. The statistical significance of the microarray-detected expression changes for all 10 genes was confirmed by qPCR. Additionally, the microarray and qPCR gene expression data showed a high correlation with in both GAL vs. STD and RL vs. STD comparisons (Table 9).

Validation of the miRNome analyses on pooled samples was performed with custom qPCR array of 14 microRNAs on individual samples. Both GAL- and RL-induced miRNA response demonstrated high correlation between pooled and individual samples $\left(\mathrm{r}_{\mathrm{GAL}}=0.92\right.$ and $\mathrm{r}_{\mathrm{RL}}=0.79, p<0.001$ ) and $85 \%$ of the miRNA changes in the individual samples were significantly different in the predicted direction $(p \leq 0.05)$, providing a strong validation for the miRNome data.

\subsection{Study 2 - A PSYChOPHARMACOLOGICAL ASSAY}

\subsubsection{Characterizing iPSCs and iNCs}

We differentiated hippocampal granule cells from iPSCs according to the protocol of $\mathrm{Yu}$ et al. which was developed on the basis of in vivo and in vitro observations on the hippocampal dentate gyrus identity and development ${ }^{63}$. Yu et al. proved that the 
differentiation protocol resulted in glutamatergic (85\%) and GABAergic (15\%) neurons, assembling the electrophysiological, receptorial and transcriptional profile of in vivo neurons. Pluripotent and differentiated cells were characterized by morphology, FACS and ICC.

6/2/F iPSCs showed stem cell like phenotype in their morphology, surface marker and pluripotent-specific transcription factor expression. Namely, FACS and ICC revealed that more than $95 \%$ of the cells expressed the stem cell surface antigen SSEA $4{ }^{97}$ and the colonies consisted of OCT3/4 and NANOG ${ }^{98}$ positive cells (Figure 6). The pluripotency of the hiPSCs was also tested in vitro when we induced free-floating EB formation.
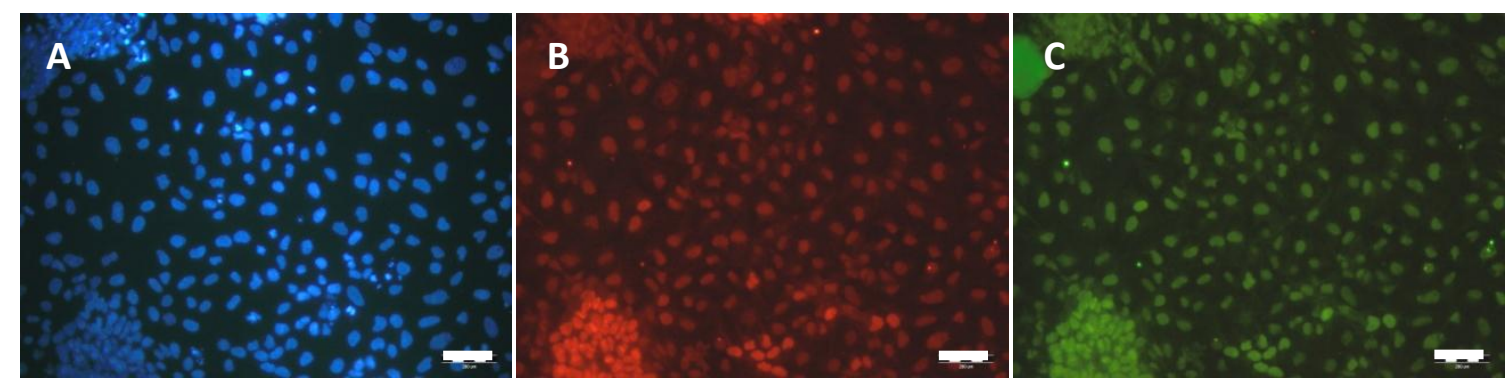

Figure 6 (A) DAPI, (B) OCT3/4, and (C) NANOG staining of iPSC cell cultures (20x; scale bar $100 \mu \mathrm{m}$ )

EBs were plated onto adherent plates and treated with N2B27 media to induce neuronal cell fate. Neural rosette-forming EBs were subcultured and treated with anticaudalizing factors (DKK1, Noggin, SB431542) and cyclopamine to provoke dorsal forebrain neural progenitor formation. Hippocampal granule cell differentiation was induced by Wnt3a and BDNF administration. After 19-21 days, cells exhibited Prox1 and MAP2 positivity (Figure 7B and C). MAP2 is a neural marker ${ }^{99}$; Prox1 is thought to be specific and essential for the survival and commitment of the DG neuronal progenitors and descendent neurons ${ }^{100,101}$.
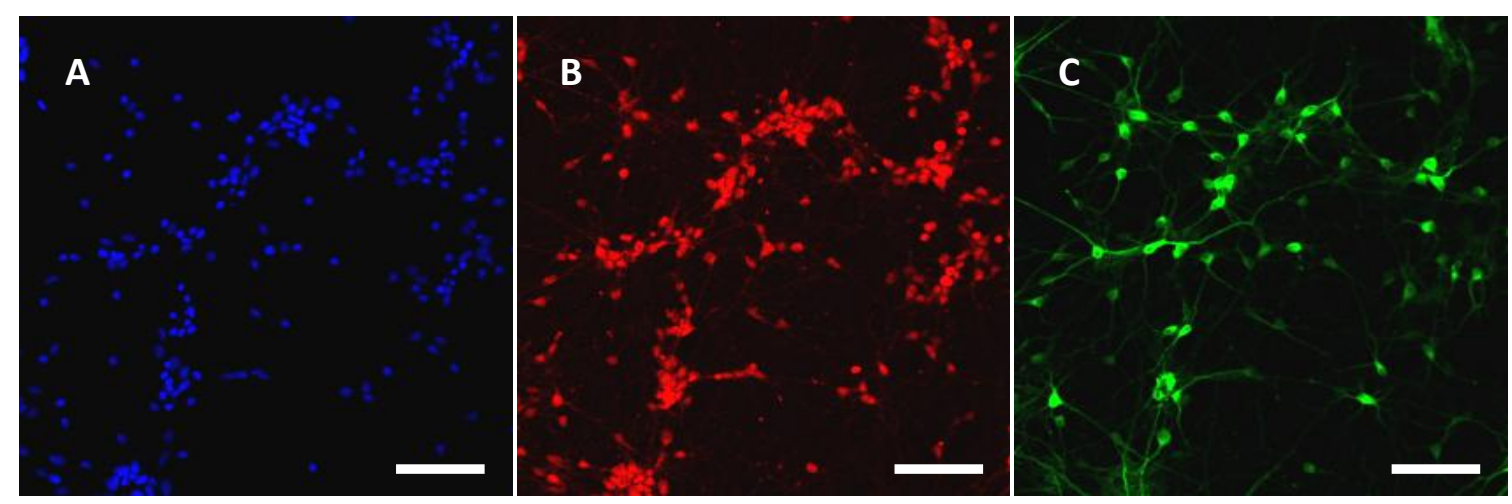

Figure 7 (A) DAPI, (B) PROX1, and (C) MAP2 staining of differentiated cultures (20x; scale bar $100 \mu \mathrm{m}$ ) 


\subsubsection{Gene expression alterations}

We aimed to examine the effect of APs on differentiating neurons; thus, we treated developing hippocampal granule cells with haloperidol (HL), olanzapine (OL) and risperidone (RP) at two different concentrations for 19 days. The expression of NEUROD1, MAP2, GFAP, $v G L U T, m G L U R 2, m G L U R 7$ genes were determined with real-time qPCR. Relative mRNA levels in the different treatment groups compared to DMSO treated control cells are represented on Figures 9-11 (note that plots demonstrate $-\Delta \Delta \mathrm{Ct}$ ).

According to our data, NeuroDl expression was significantly increased in the RP treated cells compared to CNT neurons, independently from the concentration $\left(\mathrm{RP}_{\text {low }}\right.$ and $\mathrm{RP}_{\text {high }}$ ). NeuroD1 mRNA was elevated in the $\mathrm{OL}_{\text {high }}$ and $\mathrm{HL}_{\text {low }}$ conditions $(p<0.001)$. Interestingly, HL had reverse effect at higher concentration: it lowered NeuroD1 mRNA levels compared to $\mathrm{HL}_{\text {low }}(p=0.02)$ (Figure 9).

The qPCR results showed that MAP2 transcription was augmented by $\mathrm{HL}_{\text {low }}$ treatment compared to CNT, while haloperidol exerted opposite effect at higher concentration $(p<0.05)$. MAP2 mRNA levels were not modulated by RP or OL (Figure 9).

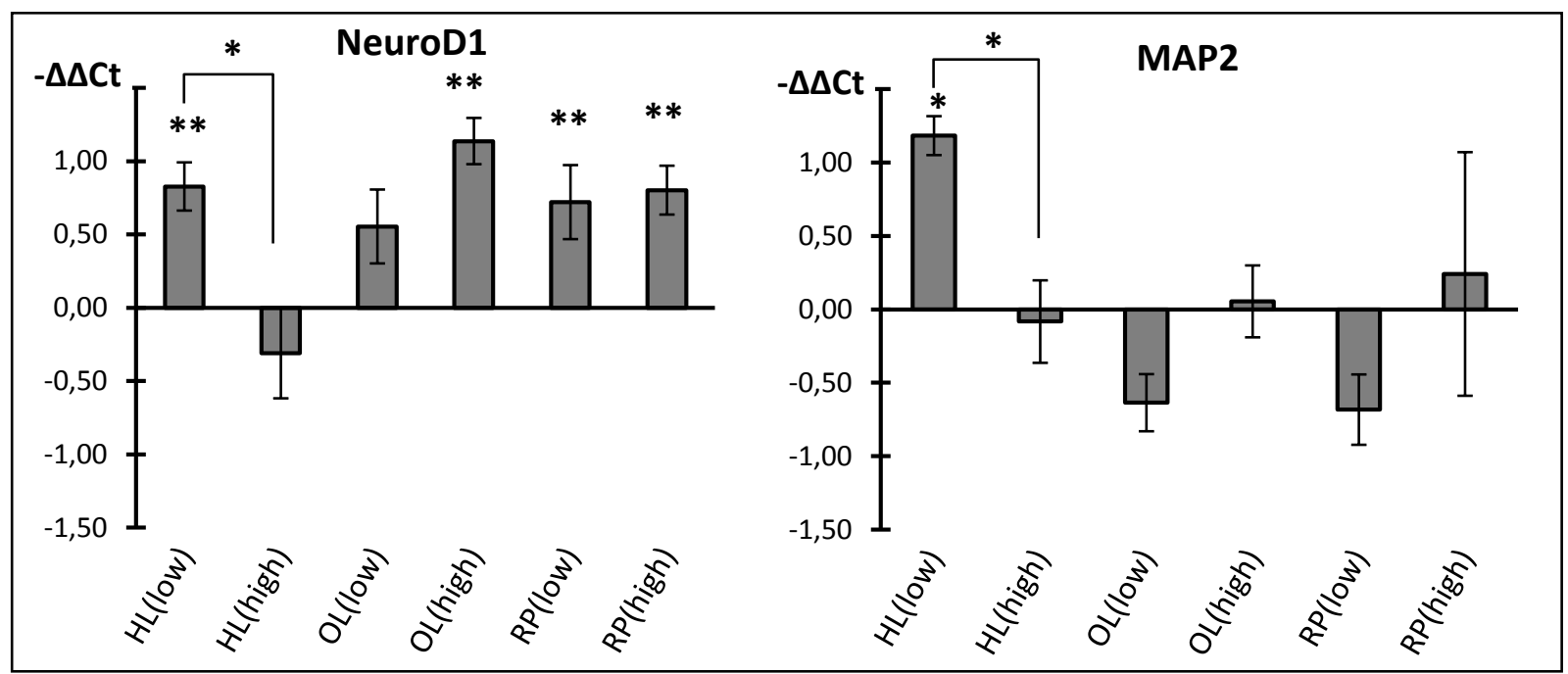

Figure 9. The relative NeuroD1 and MAP2 mRNA levels in the AP-treated cells

Interestingly, the effects of olanzapine and risperidone on GFAP gene expression were inverse at different concentrations. The AAPs enhanced GFAP transcription at low concentrations, while $\mathrm{OL}_{\text {high }}$ and $\mathrm{RP}_{\text {high }}$ depressed it compared to DMSO $\left(\mathrm{RP}_{\text {high }}\right.$-DMSO $p=0,091)$. The GFAP mRNA levels were not affected by the 19-day-long HL treatment (Figure 10).

vGLUT transcription was decreased by $\mathrm{HL}_{\text {low }}, \mathrm{RP}_{\text {low }}$ and $\mathrm{RP}_{\text {high }}$ treatments, but not altered by OL (Figure 10). 


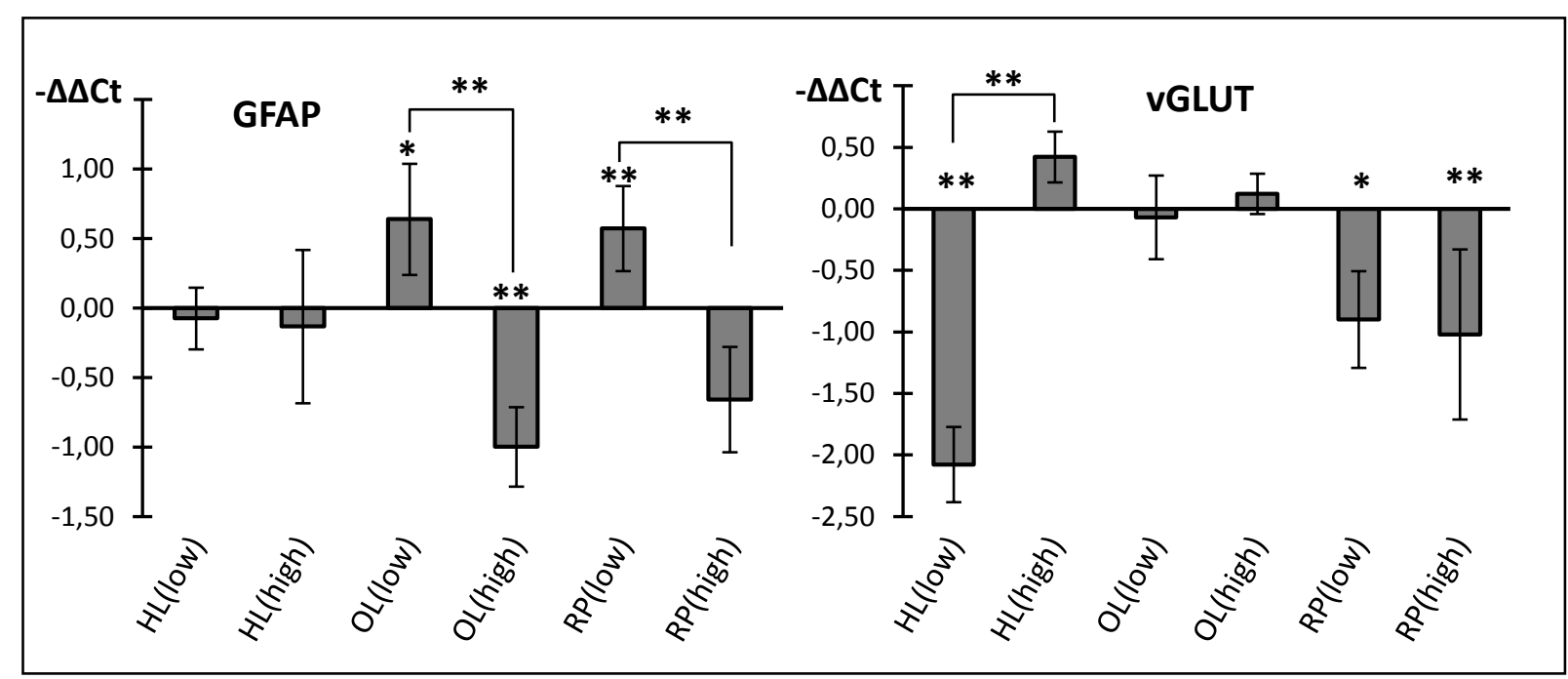

Figure 10. The relative GFAP and vGLUT mRNA levels in the AP-treated cells

Furtherly, we aimed to measure the expression levels of two metabotropic glutamate receptors, known to be expressed by hippocampal granule neurons. mGluR2 and mGluR7 were both upregulated in $\mathrm{HL}_{\text {low }}$ and suppressed in $\mathrm{HL}_{\text {high }}$. The $\mathrm{HL}_{\text {high }}$ evoked gene expression alteration was not enough powerful in comparison with control cells but proved to be statistically significant compared to $\mathrm{HL}_{\text {low }}$. Additionally, mGluR2 was underexpressed in $\mathrm{OL}_{\text {high }}$ treatment and mGluR7 in the $\mathrm{RP}_{\text {low }}$ group (Figure 11).

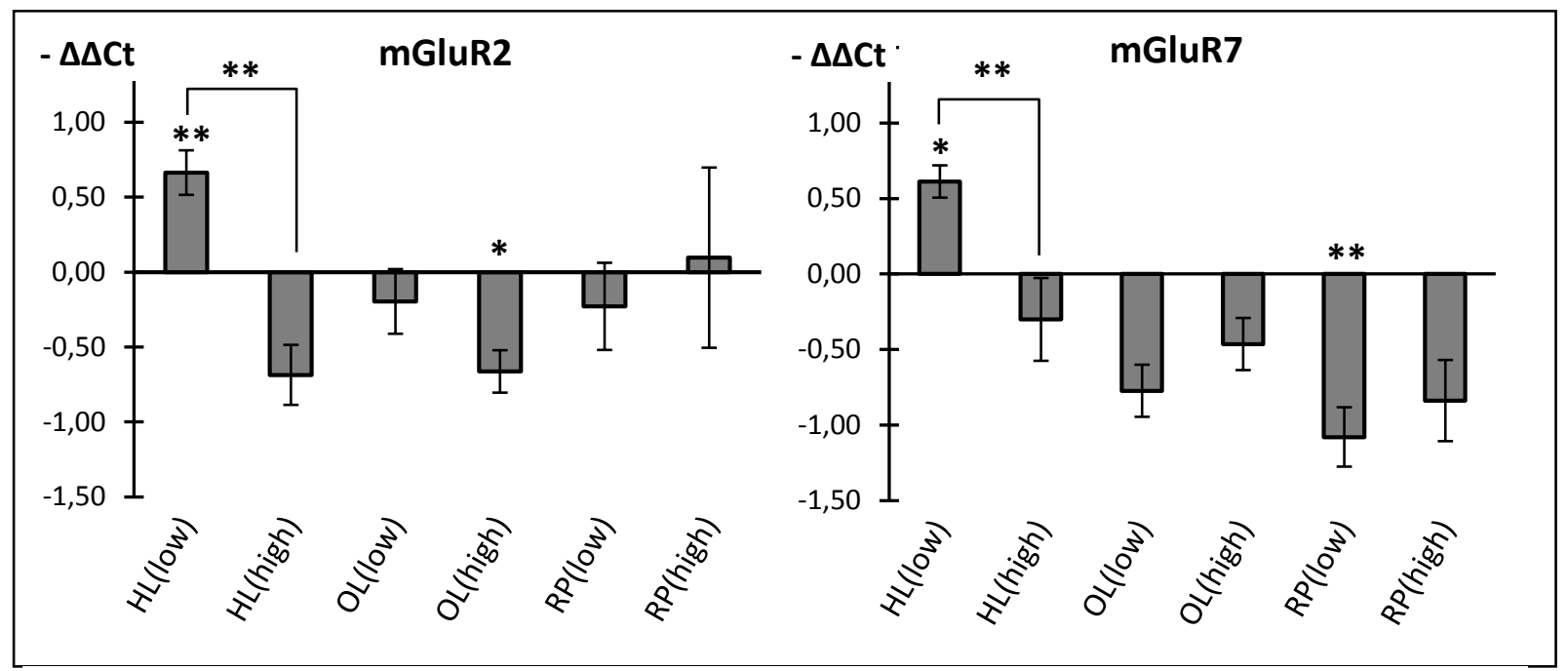

Figure 11. The relative $m G / u R 2$ and $m G l u R 7 m R N A$ levels in the antipsychoticum-treated cells 


\section{DISCUSSION}

\subsection{StUdy 1 - Diathesis-STRESS IN Fibroblasts}

Molecular profiling studies of body fluids and peripheral cells, together with postmortem findings, have already provided robust evidence for abnormalities in the immunologic/inflammatory, metabolic, hormonal, and growth factor systems in MD patients ${ }^{102}$. These findings support the concept that MD is not just a disease of the brain but instead should be viewed as a systemic disorder with molecular changes detectable across various peripheral tissues. Therefore, HDF culture might be an easily available model system with the patients individual genetic constellation.

\subsubsection{Metabolic stress response of control HDFs}

First, we analyzed the stress reponse of HDFs obtained from control subjects using two different metabolic stress conditions. We found widespread, correlating mRNA and miRNA changes.

Galactose-treatment is a widely used oxidative stress model in vitro and in vivo ${ }^{103}$. Glucose depleted, galactose supplemented (GAL) medium evokes mitochondrial stress; since, in the absence of glucose, metabolism shifts toward oxidative phosphorilation which becomes the main energy source of the cells instead of glycolysis. The mitochondrial ATP synthesis maximizes and oxygen consumption doubles, resulting in elevated reactive oxygen species (ROS) levels and compensatory overproduction of antioxidant glutathione ${ }^{104}$. Notably, glutathione regeneration requires effective adaptation and NADPH production and might be impaired by chronic nutrient depletion ${ }^{105}$.

Our observations are congruous with these since GAL-treatment provoked the activation of DNA repair and checkpoint pathways ( 9 of the 19 pathways, i.e. ATM, ATRBRCA, P53, RACCYD, SRCRPTP, G2, RB, HSP27, PPARA) and further cell-cycle related gene sets (STATHMIN, AKAPCENTROSOME).

RL-treatment (lipid reduced, cholesterol depleted medium) forces cells to synthesize their lipid and cholesterol necessities de novo, and overload the cell metabolism ${ }^{106}$. Cholesterol and other lipids are essential for the cells. Composing lipid rafts, they bias the physical characteristics of the membrane; modulate receptors, signaling pathways, influence intramembrane proteolysis and the transciption of lipid-genes ${ }^{107}$. Lipids can also act as signaling molecules or second messengers ${ }^{108}$. The lack of exogenous cholesterol intake might 
lead to the dysfunction of these essential, sensitive phenomena. Interestingly, contrary to GAL, RL-treated samples exhibited a positive enrichment of immune (TALL1, LYM, CD40) and protein or cellular regeneration related pathways (AHSP, IL22BP, TNFR2, HSP27, P38MAPK).

According to our results, while the most significantly changed mRNAs and microRNAs showed variation, the overall transcriptome changes across the two metabolic stress conditions showed considerable similarity. Based on the findings we propose that metabolic stress, regardless of the type of the insult, might have a common miRNome and mRNA signature, representing a non-specific resilience response. We identified 4 metabolic stress-related transcript pathways (PPARA, CHREBP2, RACCYCD, and HSP27) upregulated in both GAL and RL-exposed cultures, and 4 significantly changed microRNAs (miR-129-3p, miR-146b-5p, miR-543 and miR-550a) that are likely to be important mediators of metabolic stress. These common molecular consequences might originate from the same starvation-like state caused by glucose deprivation or RL. Note that in RL, cells has to increase de novo lipid synthesis, thus acetyl-CoA is used up for lipid synthesis, instead of energy production $^{109,110,111}$.

Further, the changes in microRNA expression contributed considerably to altered mRNA expression profile in both the GAL and RL conditions. The 4 commonly changed microRNAs alone appeared to be able to regulate the mRNA expression of 52 target genes, arguing that microRNA regulation of the transcriptome might be a significant contributor to metabolic stress-induced cellular responses. mir-129-3p is located next to a $\mathrm{CpG}$ island and modulated by DNA de-methylating drugs ${ }^{112}$. Hence, its expression change might indicate that (metabolic) stress alter microRNA signature through epigenetic modifications.

\subsubsection{MD-specific transcriptome}

When we compared the transcriptome profile of the MD patient derived HDFs to matched CNT fibroblasts, we found prominent changes in molecular networks associated with immune response and cell-cell interactions. According to the neurodegenerationinflammation model of MD the neuropathological alterations are resulted from immune activation and inflammation. Immune and cytokine dysregulation was detected on a molecular and tissue level in MD patients not only in the CNS but also in the periphery ${ }^{113,114}$.

However, it is also clear that the immune-related genes are only partially responsible for the genetic vulnerability of MD. The other significant component of the disease might be the disruption of intercellular communication, migration, cell cycle, apoptosis, thus 
(neuro)development ${ }^{15,116}$. Genetic elements regulating growth and organ development might also represent vulnerability factors. Our findings, i.e. enrichment of development-related gene sets, are also congruent with this view and might refer to genetically determined susceptibility since most of the epigenetic changes, environmental influences and drug effects are likely to disappear over time, as the fibroblasts continuously divide in the culture ${ }^{69}$. However, our experiments cannot exclude the possibility that some of the extremely stable, cell-division-resistant epigenetic factors might also contribute to the observed changes ${ }^{117,118}$.

An unexpected and interesting finding of our study was the strikingly different level of expression of approximately half (16 of the 37 genes) of the HGF/cMET signaling pathway. Eighty percent of the affected genes were decreased, which allow us to assume the genetically determined deficit of this pathway. c-MET is highly expressed by neurons. Hepatocyte growth factor (HGF), the ligand of c-MET receptor tyrosine kinase, is a multifunctional cytokine involved in immune regulation, neurodevelopment, adult neurogenesis and long term potentiation, processes involved in $\mathrm{MD}^{119,120}$. Previous results suggest that ischaemiaprovoked HGF production has neuroprotective effects and moderates the memory deficits ${ }^{121}$; however, the proapoptotic, antimitotic effect of MET can be suppressed by enhanced GSH production $^{93}$. Yet, the inhibition of HGF synthesis leads to depression and anxiety-like behavior in animal model ${ }^{122}$. Arnold et al. found that among plasma metabolite levels, HGF is the best predictor of the incidence and evolution of depressive symptoms in patients with age above 55 years $^{123}$. Our hypothesis, that the deficit of the HGF/MET signaling pathway might preclude the beneficial effects of this compensatory elevated HGF, might be the subject of further researches.

The patients' fibroblasts showed altered expression in a panel of 38 miRNAs. The MD-specific microRNA and mRNA profile appeared to be functionally connected, as many of the mRNAs were predicted targets of the microRNAs and changed in the opposite direction. Practically, $50 \%$ of the gene expression changes could be explain by the microRNome alterations.

The role of microRNAs in the pathophysiology of MD is under active investigation (recently reviewed in ref. 124). Depressed individuals show notable brain microRNA changes some of which are also detectable in the blood. Further, antidepressants and electroconvulsive therapy also proved to alter microRNAs. Therefore, profiling of microRNAs in peripheral samples might be potential diagnostic and therapeutic biomarkers ${ }^{124}$.

Noteworthy, at least 8 of the 38 differentially expressed microRNAs have been previously associated to neuropsychiatric disorders: miR-132 was previously related to 
BDNF-induced (patho)physiology and activity-dependent hippocampal arborisation ${ }^{125,126}$ and found to be upregulated along with decreased BDNF levels in MD patients in correlation with the disease's severity ${ }^{126}$. miR-22 regulates BDNF, serotonin receptor $2 \mathrm{C}$ (HTR2C) and monoamine oxidase A (MAO-A) ${ }^{127}$; miR-16 participate in the regulation of serotonin transporter (5HTT), mediating the SSRI antidepressant treatment response ${ }^{128}$. miR-33a-3p, and miR-425-3p were altered in the blood of MD patients ${ }^{129}$; while miR-32 and miR-22 in a postmortem bipolar dataset ${ }^{130}$. Furtherly, miR-185 was related to suicidal behavior ${ }^{131}$; miR196 and miR-7 was indicated in schizophrenia ${ }^{130,132}$. miR-429 is downregulated in response to repeated stress in rat brain ${ }^{133}$. Finally, miR-107 might be associated with accelerated disease progression in Alzheimer's disease ${ }^{134}$.

As microRNAs are considered to be potential biomarkers, more workgroups measured the microRNA levels in the blood or peripheral mononuclear blood cells (PMBCs) of MD patients. Some of our findings are congruent with these results, as HDFs exhibited MDspecific expression of miR-132, miR-103, miR-22 $2^{135}$, miR-107, miR-132 $2^{126}$.

However, the microRNAome has a poorly understood, tissue and gene expression pattern specific overall effect with notable pleiotropy and redundancy. Thus, predicted pathway analysis is elemental to contextualize the detected microRNA signature. For instance, Fan et al. detected the microRNA signature in PBMCs of MD patients and identified 22 neural function associated pathways ${ }^{136}$. Although, our diseased HDFs showed different microRNA pattern, 18 of these were also indicated in our samples (e.g. axon guidance, glutamatergic synapse, Wnt signaling pathway, ErbB signaling pathway, mTOR signaling pathway, VEGF signaling pathway, long-term potentiation, etc.).

These observations remind us to the multidimensionality of MD, i.e. the disease affects not only the CNS, and molecular anomalies are detectable in peripheral tissues. Hence, examination of fibroblasts gained from MD patients have conceptual implications for our understanding of the pathophysiology.

\subsubsection{Metabolic stress response of MD fibroblasts}

Several clinical and experimental data proved that MD and stress reaction has striking similarities on the level of organism, tissues and molecules; and stress can evoke depression in genetically vulnerable persons ${ }^{9-15}$. On the base of the diathesis-stress hypothesis of MD, we presumed that the gene expression studies could be more informative if we challenge the MD fibroblasts. In this way these molecular clues refer to the presence of genetically determined stress vulnerability even if the disordered phenotype is not detectable in STD. 
Our results demonstrate that the metabolic challenge result in robust changes of mRNA and microRNA expression. A substantial fraction of the mRNA changes, 26\% (GAL) and 33\% (RL) were observed only in MD, but not in CNT. The characteristic response of MD fibroblasts was even more pronounced on the microRNA level: 81\% (GAL) and 90\% (RL) of the total microRNA changes were MD-specific. Some of the enriched molecular pathways were already identified in the STD condition; however, metabolic stress uncovered other MDassociated impairments in the control of metabolism, energy production (FEEDER, GLYCOLYSIS) and cell migration (SPRY, ECM). Furthermore, 38\% (GAL) and 17\% (RL) of the enriched gene sets (PTC1, MPR, ACAP95, ARF, HSP27, SET, BAD, IL7, TRKA, CDMAC, SPRY, TFF) and approximately 50-60\% of the microRNAs were implicated in survival, proliferation and apoptosis. These data provide an overwhelming support for the aberrant engagement of mechanisms regulating cell proliferation and survival by MD fibroblasts when exposed to metabolic challenges.

GAL and RL treatments elicited common responses in MD fibroblasts. The CHREBP2, HSP27, BAD and ARF were the commonly enriched pathways. Three of them (CHREBP2, HSP27, and BAD) were acivated by GAL and/or RL in the CNT cells also and Arf inhibiting ribosomal biogenesis (ARF) pathway was enhanced already in STD-treated MD cells.

Both of the applied metabolic stress treatments induce starvation-like, oxidative states in the cells ${ }^{105}$. It is not surprising, that one third (GAL: 38\%, RL: 37\%) of the genes involved in the stress response of the MD-fibroblasts regulates the metabolism and energy homeostasis and both metabolic stress treatments induced miR-424 and miR-495 expression. These microRNAs are overexpressed under hypoxia and play a role in the metabolic switch from oxidative physphorilation to anaerob (glycolytic) energy production ${ }^{137,138}$.

Additionally, the RL-stress response of the MD samples varied in the expression of 8 lipid metabolism related microRNAs from the CNT samples. This fits into our previous knowledge: (1) lipid deficit is an extreme metabolic overload for cells via the forced lipid de novo biosynthesis ${ }^{106}$. (2) the dysfunction of lipid metabolism, e.g. FFA metabolism dysregulation, is a long-investigated and documented, but still poorly understood phenomena in $\mathrm{MD}^{139}$. According to our results, the RL-environment exaggerates the adaptational deficits observed in MD. We found intriguing that it induces primarily negative changes in the microRNA profile which refers to the increase of the translation and appearance of new enzyme set. 
Another interesting fact revealed only in RL-environment is the presence of six hypoxamirs. HypoxamiRs have been identified as microRNAs essential in the adaptation to oxidative stress contributing to mitochondrial respiration arrest, thus decreased production of ROSs in a low oxygen environment ${ }^{140,141}$. In our study, CNT fibroblasts upregulated six hypoxamirs (let-7b*, miR-192, miR-26a, miR-98, miR-23a and miR-7) in response to RL, whereas MD fibroblasts downregulated them. This particular difference further supports the notion of mitochondrial dysfunction in MD.

According to the microRNA target pathway analysis, both the stress-induced and MDspecific microRNA profiles are likely to affect the glutamergic synapse pathway. Evidence indicates a glutamate dysregulation in chronic stress and MD, which contributes to the neurodegeneration. Interestingly, when Gottshalk et al. investigated the protein expression profile in the prefrontal cortex of MD patients, they found that glutamate neurotransmission and energy metabolism related pathways are the most affected by the disorder ${ }^{142}$. Glutamate is a key intermediate product of energy metabolism and glutamate neurotransmission is highly linked to cellular energy metabolism (glycolysis, ATP synthesis). Our findings suggest that this in vitro stress and MD model could help us to understand the genetic and molecular background.

To prove the validity of the presented metabolic stress functional assay in the identification of MD-related gene sets, we look through the pathways which were predicted to be targeted by the stress- and MD-affected microRNAs pathways. As Figure 12 shows, the majority of MD-specific pathways (green) were also modulated by the challenge-evoked microRNA signature. 


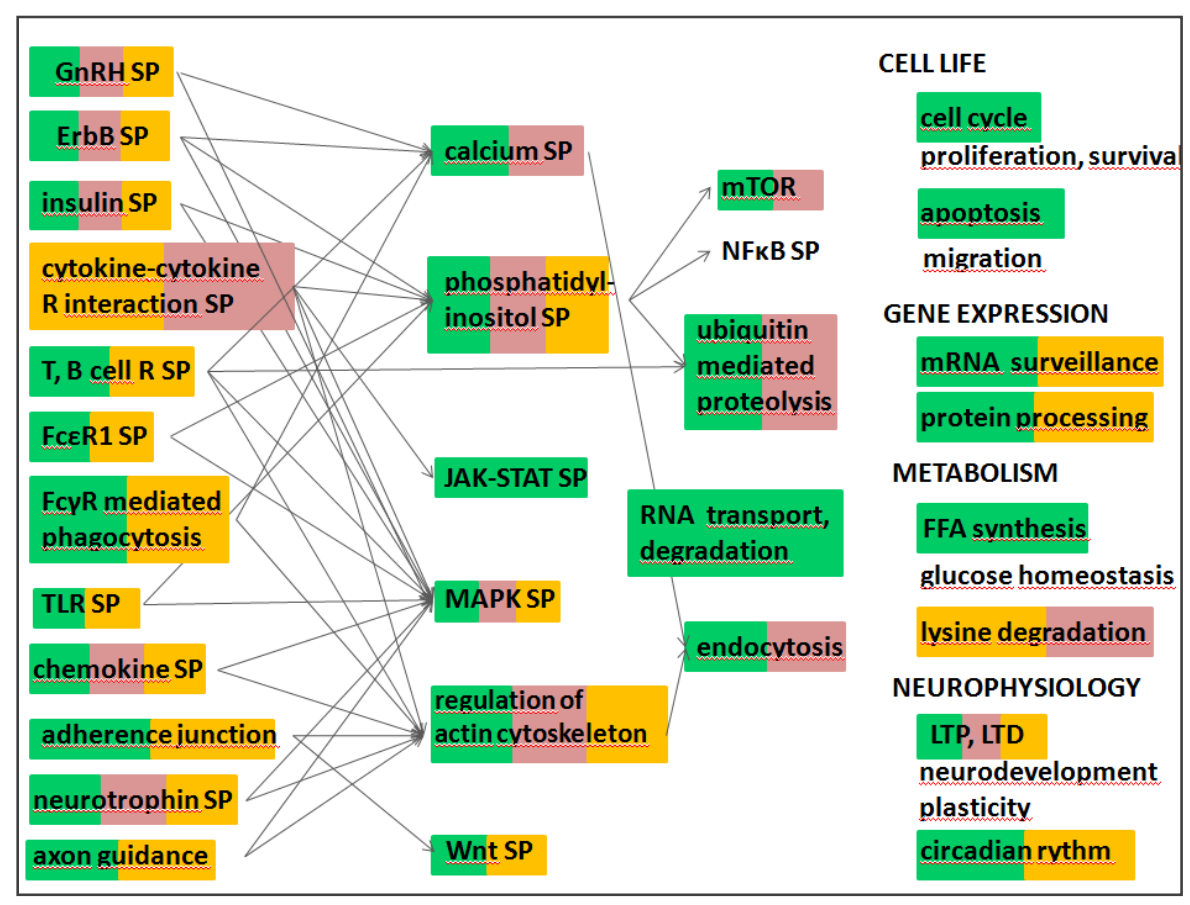

Figure 12. Majority of the stress-induced and MD-associated microRNome target pathways. (green indicates MD-specific target gene sets in standard conditions; yellow highlightes the stressinduced pathways in CNT; purple signes the challenge-induced pathways in MD cells)

An important question is whether the changes we detect in peripheral tissues are relevant to a disease process that takes place primarily in the brain. Searching previous literature, we found that the challenge-induced transcriptome changes of MD cells can be linked to neural functions. For instance, genes important for neurite outgrowth, neurodevelopment and synaptic plasticity were identified: actin-related protein $2 / 3$ complex, subunit 5 (ARPC5) $)^{143}$, ArfGAP, ankyrin repeat and PH domains 2 (ACAP2) ${ }^{144}$ and beta-1,3glucuronyltransferase $2(B 3 G A T 2)^{145}$. Multiple important roles in the CNS are associated with the microRNA stress-response signatures as well. Indeed, 31\% (GAL) and 47\% (RL) of the stress-affected microRNAs are indicated as regulators of neuropathophysiology or biological markers associated with psychiatric disorders. miR-7 ${ }^{146}, \operatorname{miR}-26 \mathrm{a}^{147}$, miR$34 \mathrm{a}^{148}$, miR-134 ${ }^{149}$ and miR-132 ${ }^{150}$ participate in neurite outgrowth and synapse formation. miR-25 ${ }^{151}$, miR-34a ${ }^{152}$, miR-497 $7^{153}$, miR-196a and miR-376a are involved in cell cycle, apoptosis and neural differentiation ${ }^{154}$. miR-7 and miR-370 ${ }^{155}$ regulate brain morphogenesis; while miR-23a influences myelination ${ }^{156}$. Furthermore, we detected some microRNAs which were previously named as biomarkers in schizophrenia (miR-132, miR-564 and miR-382 ${ }^{157}$ ); Alzheimer's disease (hsa-let-7g, miR-15b ${ }^{158}$ ); Huntington's disease (miR-1285, miR-34a and miR-34b ${ }^{159}$ ). A number of miRNAs that respond to different stressors in the CNS: miR-34b, which affects the degree of oxidative stress and survival of dopaminergic neurons ${ }^{160}$; miR-296-5p, which responds to oxidative stress in mouse hippocampal neuronal 
cultures $^{161}$; miR-199a, which reacts to hypoxia in rat cortical pericytes ${ }^{162}$; and miR-98, which is elevated in the brains of newborn rats experiencing prenatal stress ${ }^{163}$.

Taken together, the information gives confidence to the relevance of our observations in fibroblasts to the MD disease state. We can conclude that presenting metabolic challenges were instrumental for defining distinct MD mRNA and microRNA response signatures that might also contribute to patients' aberrant responses to life stressors.

\subsubsection{Never-ending limitations}

Considering study design; utility of HDFs in psychiatric research; or difficulties of transcriptome studies, several limitations have to be acknowledged when evaluating our results. Although our sample size was determined to ensure enough statistical power for microarray screening and we performed repeated measures, our results would require validation in larger cohort of MD patients to regard the detected transcriptome changes as potential biomarkers.

Expanded literature suggests that MD is a heterogenous systemic disease without clear, consensual subtyping. Several (epi)genetic, neurobiological, neuroimaging characteristics were found only in a subset of patients hence the differences could be masked in the total depressive patient population, underscoring the value of diagnostic subclassification (e.g. serotonin signaling pathway - melancholic depression ${ }^{68}$ ).

There are several drawbacks that should be considered when using the HDF experimental model. Several research groups investigated the in vivo and in vitro cellular aging-related changes in HDFs. They revealed that mRNA, miRNA and protein levels ${ }^{164}$, DNA methylation ${ }^{165}$, intracellular calcium levels, mitochondrial function and cell size ${ }^{166}$ are all influenced by the age of the donor. These findings necessitate careful case-control age matching and/or use of senescence markers.

Furthermore, matching the number of passages is equally important to avoid consequences of in vitro cellular ageing i.e. mitotic rate, telomere length, DNA methylation ${ }^{20}$, proteome and protein phosphorylation ${ }^{167}$ changes that occur after the P15 in HDF cultures. The cells enter replicative senescence between $\mathrm{P} 21$ and $\mathrm{P} 40^{20}$. Additionally, the early generations might also undergo significant transcriptome and epigenome remodeling during the adaptation to the culturing conditions ${ }^{168,169}$.

Even though HDFs are thought to lose most of the epigenetic footprints after 5 passages ${ }^{69}$; and Fournier et al. underpinned that body mass index (BMI), smoking and medication do not influence the metabolome of HDF cultures past several passages ${ }^{22}$, we 
decided to perform our experiment on HDFs between P5-P10. For microRNome analysis, we pooled the samples regarding age and gender and the results were validated with individual samples. However, we cannot exclude the possibility that the lack of clinical information (i.e. received medication) might hinder clear conclusions.

Lastly and obviously, HDF cells are not brain cells: they are much more robust, stressresilient with self-renewal capacity ${ }^{18}$. The energy homeostasis of neurons relies on lactate oxidation thus, they live in a symbiotic relationship with glial cells ${ }^{170}$. Additionally, neurons are specialized to rapid cell-cell communication, while HDFs are primarily stromal cells with a prominent role in immune response and regeneration ${ }^{171}$. We have to be aware all these differences when putting our results in context of the diathesis-stress model of MD.

There are several problematic issues on microRNA-studies as well. First of all, target mRNAs are identified with computational methods and not necessarily validated in vitro or in vivo. Since microRNAs bind to target mRNAs via imperfect complementarity, understanding exactly which target mRNAs are recognized by which specific miRNAs is challenging ${ }^{172}$. The mechanism, how microRNA regulates protein synthesis is not as simple and obvious as previously thought (a simple negative effect) ${ }^{29,31}$. Additionally, translation regulation is not the only biological function of these pleitropic molecules: they also serve as paracrine, endocrine signaling molecules ${ }^{26}$. Thus, further efforts are needed to establish connections between microRNA and mRNA expression data, improve target gene and pathway prediction. In regard of these and the huge amount of data, we carried out pathway analysis to get a clue what are the overall consequences of the detected transcriptome profile changes in MD and CNT HDFs.

\subsection{StUdy 2 - A PSYChOPHARMACOLOGICAL ASSAY}

We used a special differentiation protocol resembling adult hippocampal neurogenesis and resulting in granule cells to study the AP-induced gene expression changes. Importantly, the same model system was recently applied in a Lithium-response assay ${ }^{52,173}$. Hippocampal neurogenesis is mandatory for the maintenance of normal cognition through the life. Exogen factors impairing it (e.g. chronic stress or radiation) have detrimental effects on cognition and $\operatorname{mood}^{174}$. Thus studying neurogenesis during AP treatment have implications for the large community of patients who take these medication for years or decades.

Haloperidol (HL) is a typical antipsychotic drug (TAP) acting primarily on D2 dopaminergic receptors with lower activity at D1, D3, D4, 5-HT2A serotonergic, and $\alpha 1$ adrenergic receptors (pharmacodynamics of APs reviewed in: ref. 175). In addition, it alters 
NMDA binding and glutamate transporter expression thus exert a hyperglutamatergic effect $^{176,177}$. In contrast, olanzapine (OL) and risperidone (RP) are atypical antipsychotics (AAPs) which operate primarily as 5HT2A antagonists and relatively weak D2 antagonists. Furtherly, they block with moderate affinity several other serotonergic, dopaminergic, histaminergic and alfa-adrenergic receptors ${ }^{178}$. Clinical studies suggested that treatment with AAPs, may slow the progressive changes in brain structure in patients with schizophrenia ${ }^{179}$.

We have to take into account that APs might have poorly known off-target effects. For example, HL can block L- and T-type calcium channels, enhance voltage-gated sodium channel expression thus affect the generation and propagation of action potentials ${ }^{180,181}$. Similarly, we must not forget that deducing from in vitro data to clinical implications is possible only a very limited way and has to be done cautiously.

\subsubsection{Neuronal differentiation 1 (NEUROD1)}

According to our results, low and high concentration RP and high dosage OLtreatment increased the expression of NeuroD1. HL exerted augmenting effect on the gene at lower concentration, but not at higher.

NeuroD1 is a transcription factor, key regulator of vertebral neurodevelopment. NeuroD1 expression diminishes as the brain matures except in the cerebellum and hippocampus where it controls the differentiation of granule neurons throughout adulthood via activation of E-box containing gene promoters (e.g. IGF1, NCAM and TrkB) ${ }^{182,183}$. NeuroDl activation or transfection is used to induce neural fate in pluripotent cell cultures and forced expression of NeuroDl alone have promoting effects on neuronal differentiation ${ }^{184}$. Hsieh et al. found that NeuroD1 overexpressing NPCs differentiated into neurons $(>75 \%)$ and reduce their ability to develop oligodendrocytes and astrocytes ${ }^{185}$. Agents (e.g. retinoic and valproic acid) increasing NeuroD1 levels are known to promote neural differentiation and inhibit astroglia and oligodendroglia development ${ }^{185,186}$.

We have to consider that NeuroD1 elevation in the treated versus untreated cultures can be indicative of accelerated neurogenesis or augmented survival as well, in general. However, the bFGF deprivation from the first day of AP-treatment had to arrest proliferation; thus, we assume that APs facilitated cell survival only at certain concentrations. These findings are aligned with the previous literature. The beneficial effect of APs have been attributed partially to their capacity to increase neurogenesis/survival; however, the results are often inconsistant especially in case of HL (reviewed in ref. 187 and 188). 
On the whole, OL administration repeatedly proved to increase the number of newborn cells with neural fate in the rodent dentate gyrus ${ }^{189}$ and improve memory performance via increasing brain-derived neurotrophic factor (BDNF) levels in the hippocampus ${ }^{190}$. Others suggested neuroprotective rather than proliferative effect of $\mathrm{OL}^{191}$.

Most of the studies were not able to detect any consequences of RP and HL-treatment on hippocampal cell proliferation ${ }^{187,188}$. On one hand, RP and HL both found to promote the survival of young neurons in the hippocampus by anti-apoptotic BCL-2 and VEGF induction ${ }^{192}$, on the other hand significant body of data suggests detrimental effect of $\mathrm{HL}^{193,194,195}$ and several publications discussed the neuroprotective potential of AAPs above the TAPs (usually OL>RP>HL) ${ }^{195,196,197}$. One possible mechanism of the dual HL-effect is dose-dependency ${ }^{193,198}$ what was confirmed in our results also: there was a significant difference between $\mathrm{HL}_{\text {low }}$ and $\mathrm{HL}_{\text {high }}$.

This great diversity of data might be due to differences in the dosage, application regime, examined brain areas and animal models ${ }^{192}$. For instance, Green et al. reported that OL increased cell proliferation in the prefrontal cortex and subventricular zone but not in the dentate gyrus ${ }^{199}$. There might be major variaties within circuits since the depletion of neural transcription factors (NeuroD1, neurogenin 1, 2 or 3) induces massive apoptosis of DG granule cells but does not affect CA regions seriously ${ }^{182}$.

The effects of AP treatment on neurogenesis/survival is especially worthy of further research: schizophrenia can be referred to as a neurogenesis deficit disorder since neurodevelopmental and/or adult neurogenesis, in particular, DG neural stem cell proliferation might be deficient ${ }^{200}$.

\subsubsection{Microtubule-associated protein 2 (MAP2)}

We found that MAP2 expression was altered in the differentiating neurons only by haloperidol: the 19-day-long $\mathrm{HL}_{\mathrm{low}}$-treatment increased the relative mRNA levels, while AAPs did not affected it.

MAP2, as other microtubule associated proteins, promotes microtubule assembly, polymer stabilization and regulate organelle transport. Since it is predominantly expressed in neurons, it is used as an (early) neural differentiation marker in stem cell neural differentiation assays $^{201}$. More importantly, MAP2 represents a specific neurite outgrowth marker also participating in dendrite spine formation and neuronal plasticity in vivo and in vitro ${ }^{202,203}$. $M A P 2$ expression alterations are usually evaluated as increased synaptic plasticity, and 
thought to reflect functional and/or structural changes in the dendritic tree ${ }^{204}$. Accordingly, our findings suggest that $\mathrm{HL}_{\mathrm{low}}$ treated cells exhibited active synaptic dynamics.

Large body of data underpins that APs alter synaptic morphology and expression of several presynaptic genes in addition to their classic receptorial mechanism. In deed, enhanced neural plasticity and synaptic remodeling thought to be important detail of their therapeutic mechanism 205 , 204. However, notably few study examined the postsynaptic molecular effects of $\mathrm{APs}^{204}$.

Lidow et al. showed increased MAP2 phosphorilation after long-term haloperidol treatment in primate cortex but did not detected expression changes ${ }^{206}$. An other study measured the effects of 14-day-long HL, RP and OL on the cortical and hippocampal MAP2 mRNA levels. The gene expression was increased in the rat cortex by HL and RP and in the dentate gyrus by RP and OL but remained unchanged in CA1-3 $3^{204}$.

Yet, it is worthy of note that gene expression is only one part of MAP2 function regulation: the binding and activity of MAPs depend on their phosphorilation state and MAP2 serves as substrates for most of protein kinases and phosphatases present in neurons (PKA, PKC, ERKs, CAMKII, GSK3, CDK, MARKs) ${ }^{203}$. Hence, our observation that RP and OL do not modulate the gene expression itself is not in contradiction with previously detected APinduced plasticity, arborisation changes.

Contextualizing our results, we must also not forget that synaptic changes are modulated on a network level which is per se much more complex in vivo than in vitro. Our study implies that RP and OL does not influence directly the MAP2 expression in cultured neurons contrary to HL. This is aligned with previous findings, suggesting that TAPs and AAPs regulate differentially the dendritic spines and postsynaptic plasticity ${ }^{207}$. Appreciating the known dendritic pathology in schizophrenia (e.g. decreased hippocampal MAP2 levels ${ }^{208}$, ${ }^{209}$, but see ${ }^{210}$ ), these molecular consequences are worthy of further investigations.

\subsubsection{Glial fibrillary acidic protein (GFAP)}

Interestingly, we found that AAPs exerted inverse effects on GFAP expression at different concentrations: OL and RP elevated GFAP mRNA levels at low concentration but repressed it at higher dosages.

GFAP is the major intermediate filament of mature astrocytes and has been widely used as an astrocyte differentiation marker ${ }^{211}$. Elevated GFAP level can refer either to astrocyte activation or increased number of glial elements ${ }^{212}$. The phenomenon that differentiating neural cultures can develop glial cells spontaneously was evidenced by several 
research groups who detected non-neuronal cells in stem cell-derived neural cultures using various differentiation protocols (data vary between $6-50 \%^{213,214,215,216}$ ). These cells include neural and glial precursors, oligodendrocytes and GFAP positive astrocytes ${ }^{213}$.

Even though, alterations in levels of GFAP may reflect pathological regulation of neuronal function, survival, as well as synaptogenesis and neurotransmission, studies on astrocytes in context of psyciatric disorders are underrepresented in neuroscience. For instance, directed differentiation of glial cells from human PSCs has not been paid sufficient attention since glia are regarded as supporting cells and appearance of glia in the differentiating neural cultures as a by-product default consequence of differentiation ${ }^{217}$. However, astrocyte abnormalities have been detected in schizophrenia, depression and bipolar disorder in various brain regions ${ }^{218,219}$. Among these, the most commonly reported gene expression alteration is reduced level of $\operatorname{GFAP}^{218,187,220}$ which is further modified by antidepressant and AP-medication ${ }^{221,222}$.

Despite of these, surprisingly few study aimed to investigate the effect of APs on the expression of GFAP: searching PubMed for haloperidol OR olanzapine OR risperidone AND GFAP, we found 6 relevant papers. Importantly, the results suggest that GFAP-modulatory effect of APs might be human and subregion specific ${ }^{223,222}$. For instance, Radomska et al. found that GFAP expression of human primary astrocytes were increased by $6 \mathrm{~h}$ HL-treatment in vitro ${ }^{223}$; and others detected OL-induced GFAP upregulation and proliferation suppression in human glioblastoma cells ${ }^{224}$. The workgroup suggested that the mediator might be a human-specific Quaking (QKI) -binding element in the gene promoter ${ }^{223}$. This might explain the negative findings in rodent models after HL, RP and OL treatments $225,212,226$.

The effect mechanism of APs on glial cells is poorly known. Notably, astroglia response to antidepressants and thought to be key factors of their beneficial effects on neurogenesis ${ }^{227}$. They express wide range of neurotransmitter receptors in a brain regionspecific manner (reviewed in ref. 228 and 229). For instance, hippocampal astrocytes were reported to express serotonine (5HT1A, 2A, 2B, 2C), glutamate (AMPA, mGluR1-8 except mGluR7), $\mathrm{GABA}_{\mathrm{A}}$ and $\mathrm{GABA}_{\mathrm{B}}$, muscarinic, beta adrenergic, histamine, and somatostatine receptors; more of them modulated by APs. In addition, several signaling molecules proved to influence the transcription of GFAP, e.g. circulating cytokines, endocrine hormones, neuronreleased glutamate and TGF- $\beta$.

Overall, while our findings and previous data support a role for GFAP in pathomechanism of APs, the exact nature of this, and the pathological implications of these findings remain unclear. 


\subsubsection{Metabotropic glutamate receptors (mGluR2 and mGluR7)}

We found that the effect of APs on $m G l u R 2$ and $m G l u R 7$ genes depended on the dosage: both receptors were upregulated by $\mathrm{HL}$ at low concentration. In contrast, mGluR2 and mGluR7 mRNA levels were decreased by $\mathrm{OL}_{\text {high }}$ and $\mathrm{RP}_{\text {low }}$, respectively.

Metabotropic glutamate receptors (mGluRs) have various modulatory functions on neurotransmission and synaptic plasticity especially in hippocampal learning and memory processes ${ }^{230}$. The eight mGluR subtypes are classified into three groups: group I (mGluR1/mGluR5) coupled to inositol phospholipid hydrolysis, group II (mGluR2/mGluR3) and group III (mGluR4/mGluR6/mGluR7/mGluR8) linked to inhibition of the cAMP cascade $^{230}$. Group II and III mGluRs restrain glutamate release by inhibiting pre-synaptic voltage-dependent calcium channels and/or interfering with the release-system ${ }^{231,232}$.

mGluR2 is predominantly expressed in dentate granule cells and distributed specifically on the mossy fibers at the preterminal zone or presynaptically ${ }^{231}$. It is an actively studied new therapeutic target in schizophrenia ${ }^{233}$, anxiety, depression ${ }^{234}$, and substance use disorders $^{235}$.

According to animal and human postmortem data, chronic RP, OL and clozapine treatment, but not haloperidol, downregulates $m G l u R 2$ expression ${ }^{236,237}$. Importantly, the silencing effect of AAPs is modulated by the decrease of histon $\mathrm{H} 3$ acetylation and enclosing the promoter of $m G l u R 2$ among other genes ${ }^{237}$. Furtherly, this repressive histon modification proved to be serotonin $2 \mathrm{~A}$ receptor $(5 \mathrm{HT} 2 \mathrm{~A})$ dependent unravelling the ineffectivity of HL: HL is a silent agonist of 5HT2A, while AAPs (as RP and OL) are high affinity antagonists $^{237,238}$. 5HT2A was detected in the granular layer of dentate gyrus and pyramidal cell layer of CA1-CA3 of the hippocampus ${ }^{239}$. Our data suggest that HL might evoke mGluR2 overexpression through an other, currently unknown mechanism in a concentrationdependent manner what could be interesting subject for further investigations.

Further, the glutamatergic hypothesis of schizophrenia, clinical and preclinical observations suggest that mGluR2 activating drugs represent potentially new AP medications 233-235, 240. Therefore, some speculate that $m G l u R 2$ downregulation might restrain the therapeutic effects of existing AAP drugs ${ }^{237}$.

Genetic association studies identified $m G l u R 7$ as a candidate gene for schizophrenia, bipolar disorder and other psychiatric disorders ${ }^{241,242}$. The schizophrenia-linked genotype might result in lower mGluR7 expression; although, studies on mGluR7 levels in patients have not been reported ${ }^{243}$. Recently, the receptor gained significant attention when proved to be more than an inhibitory presynaptic autoreceptor ${ }^{244,245}$. Indeed, mGluR7 is also expressed 
presynaptically on the GABA-neurons of the hippocampus and basal ganglia. It suppresses GABA transmission thus enhances LTP in the hippocampus and disinhibits the nigrostriatal dopaminergic pathways ${ }^{245,246}$. Additonally, agonists may have neuroprotective effects in ischaemia and excitotoxicity ${ }^{247}$. As a consequent, it is hypothesized that lower expression of mGluR7 may increase risk of developing schizophrenia, have potential therapeutic implication in cognitive impairment and extrapyramidal movement disorders, especially those originating from AP-induced dopamine depletion ${ }^{248,249}$.

Notably, despite of its potential involvement in neuropsychiatric disorders and the active research on novel mGluR7 acting drugs ${ }^{250,247}$, we did not find any publications about the effects of HL, RP, OL or other APs on mGluR7 expression or function.

\subsubsection{Vesicular glutamate transporter 1 (VGLUT1)}

We found that $\mathrm{HL}$ at low concentration and RP at both concentrations decreased the expression of VGLUT1. This protein, encoded by the SLC17A7 gene, orchestrates the synaptic vesicle cargos: its expression pattern directly influences glutamate release (i.e. glutamate quantal size $)^{251}$ and correlates with learning and memory processes ${ }^{252}$. Accordingly, VGLUT1 plays a pivotal role in glutamate release, synaptic plasticity, postnatal neurodevelopment and learning 253,254 . Downregulation is implicated in the cognitive symptoms of affective and neurodegenerative disorders ${ }^{254}$. Further, VGLUT1 is a presynaptic marker of excitatory neurons in the cortex and hippocampus ${ }^{255}$.

Importantly, VGLUT1 expression modulation might be region, furthermore cortical layer specific and age-dependent, thus published results are often inconsistent ${ }^{256,257}$. In general, postmortem and animal studies revealed altered glutamate neurotransmission and VGLUT1 expression in MD, bipolar disorder ${ }^{256,258}$, and schizophrenia ${ }^{259,257}$. Uezato et al. reported that HL interferes with VGLUT1 in the temporal cortex of rats but not in the hippocampus $^{256}$; while others found no effect of HL on VGLUT1 ${ }^{257}$. Clozapine (AAP), lithium and antidepressants raised VGLUT1 in the cortex and hippocampus ${ }^{260}$. Notably, we found no previous data on the effect of RP or OL on VGLUT1.

\subsubsection{Limitations and future directions}

Although, iPSC/iNC cultures are probably the most promising tools in brain-research, they have considerable shortcomings. First of all, even if the cell lines can be initiated from easily obtainable biospecimens, the reprogramming, culturing and differentiation is laborintensive and requires notable expertise ${ }^{261}$. Proliferating iPSCs exhibit genetic instability which may result in population diversity and biases in genetic assays ${ }^{262,263}$. 
Although, the hereby used monolayer technique thought to be the most efficient way to synchronize and control NPC development in vitro ${ }^{264}$. However, iPSC-derived neural cultures always contain progenitors, glial cells and mature or immature neurons with different neurotransmitter and receptor profiles and varying electrophysiological properties ${ }^{213}$. For instance, $\mathrm{Yu}$ et al. reported that the above described protocol resulted in predominantly glutamatergic neurons $(>85 \%)$ with low occurrence of GABAergic neurons $(<15 \%)$. Additionally, we detected GFAP-response to AP-treatments which would be impossible in a neural culture theoretically. On one hand, this resembles better the physiological, in vivo conditions suggesting more natural cell functions. On the other hand, it might necessitate single-cell molecular-biology approaches ${ }^{213}$.

Using iNCs, we aimed to study the effect of APs on neurogenesis and differentiating hippocampal granule cells. However, we have only limited knowledge of how far can we mimic in vivo embryonic or adult neurodevelopment. NPCs and new neurons need a special niche, surrounded by glia, blood vessels, and ependymal cells ${ }^{55}$. They follow a stereotypic process for network integration in close interactions with hundreds of cells and different excitatory and inhibitory signals ${ }^{265}$. Still, the maturation process (i.e. reaching electrophysiological, neurochemical properties similar to adult neurons) might last for 6-10 weeks in vitro ${ }^{52}$ that can be shortened by growing the neurons on feeder astrocytes ${ }^{266}$. In conclusion, when we decided to use poor, monolayer neuronal cultures, we compromised between subideal techniques considering pros and contras and resourcements.

Importantly, epigenetic effects of APs and other psychiatric medications are under active research. iNCs might be key approaches of these studies as iPSCs undergo epigenetic rearrangement during reprogramming and differentiation. This might be a notable advantage since cells are likely to lose in vivo effects, e.g. lifestyle or medication. However, the inherited and early epigenetic effects are also erased from the functional assays. Furthermore, iPSCs exhibit a considerably high number or epigenetic abberrations ${ }^{267}$.

One of the weakest point of our experiment might be the concentration of APtreatments. When choosing the dosage, we had to consider two major issues: we aimed to model in vivo circumstances (i.e. the therapeutically-relevant serum concentrations) and gain results comparable with previous data. Yet, contextualizing our results might be problematic due to difficulties in establishing dose equivalences between APs, poor information on real brain concentrations and disputed dosage in animal studies ${ }^{268,269}$. Correlating receptor occupancy in vitro and in vivo might improve the validity of these pharmacological assays ${ }^{269}$. 


\subsection{OUR MAJOR FINDINGS}

In the first study, we aimed to set up and test an in vitro functional assay for modeling MD in the diathesis-stress aspect. Here, we suggest that the above presented results correspond with the hypothesis that MD is a genetically determined, systemic maladaptational disorder.

(1) First, we wanted to know how metabolic stress-response manifests on the level of mRNAs and microRNAs in HDFs? Our results suggest that metabolic stresses resulted in similar, but not identical, robust transcriptional changes affecting gene sets implicated in cell cycle, apoptosis, inflammation, and metabolic adaptation. The microRNA signature contributed considerably to the mRNA stress-response.

(2) Next, we examined if there are any differences between the MD and CNT samples in the mRNA and microRNA profile? We found a functionally interconnected microRNAmRNA network disturbance in MD cells: namely, a loss-of-function genetic dysregulation with most prominent decreases in genes related to cell communication, adhesion, immune functions and apoptosis.

(3) Finally, we compared the stress response of MD and control HDFs. These data underpin our hypothesis that the dysregulated microRNA profile in MD contributes to the maladaptation. We can also assume that MD cells can reach the required metabolic changes via non-physiological ways what result in increased vulnerability. Contrary to CNT cells, RLmedium showed to be greater challenge for MD cultures. Understanding this phenomena and investigating the MD-specific stress response might help to get closer to the genetic background of maladaptation.

In Study 2, we established an in vitro pharmacological assay using neural cells originated from human iPSCs and treating them with typical AP haloperidol or with atypical olanzapine and risperidone. As far as we know, we are the first who examined the effect of APs on iPSC derived neural cells during differentiation and maturation.

AAPs increased the expression of NeuroD1, indicative of accelerated neurogenesis and/or augmented neural survival. MAP2 transcription was elevated by $\mathrm{HL}_{\text {low }}$ suggesting increased synaptic plasticity, and functional-structural changes in the dendritic tree. Our results call attention to the existing, but different effects of APs on the expression of $m G l u R s$, which are actively studied new therapeutic targets in psychiatric disorders and implied in

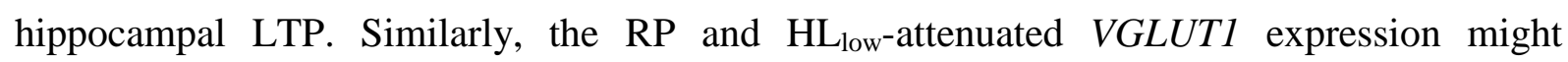
contribute to the understanding of cognitive aspects of long-term AP-treatment. Interestingly, GFAP was modulated by AAPs which can be remarkable subject of further research. 


\section{CONCLUSIONS}

On the basis of our observations, we argue that in vitro model systems have potency in psychiatric research.

First, we worked with the classic, but still valid patient-derived HDFs which have been utilized in psychiatric studies since $1978^{270}$. We presented that the mRNA and microRNA profile of the cells gained from MD and CNT subjects are different and reflect to the known MD-specific CNS and peripheral pathophysiological phenomena. Previously HDF cultures were used to study the signal transduction alterations, oxidative stress in $\mathrm{MD}^{271,272}$. However, we are the first, who used these peripheral cells to get familiar with the (epi)genetic regulation of stress reaction and MD. Our research might serve as a base to develop a new stress model, which allows us to discover the (epi)genetic risk factors and $\mathrm{G} * \mathrm{E}$ in the background of maladaptational disorders, such as MD.

Second, we used iPSC-derived neural cells, one of the most promising models in current neuropsychiatric research. We found AP-induced gene expression alterations in differentiating hippocampal granule neurons. Considering our and previous results, we have to acknowledge that APs exert complex, poorly understood modulatory effect on the gene expression and cell pathophysiology in a brain region- and cell type-specific manner; therefore, these cannot be studied in peripheral cells. Understanding these mechanisms might have direct consequences in clinical and experimental pharmacology. 


\section{ACKNOWLEDGEMENT}

First of all, I wish to express my thanks to my tutors: Prof. Károly Mirnics who taught me the importance of sharing and esteeming views and opinions during scientific work, and Prof. Zoltán Janka who encouraged me to be a precize, careful researcher with deliberate aims. Without their unceasing critical support and assistance throughout the past years, this research work and thesis would have never been accomplished.

I also express sincerely gratitude to János Réthelyi, who allowed me to join their research work where I have learned the significance of being patient and mindful of our limitations.

I am using this opportunity to say thanks to Magdolna Pákáski. Her professional and personal assistance and advice helped me through several difficulties during the past years (and currently as well). I am also indebted to Krassimira Garbett and Edit Hathy who were always there to enjoy peaks and overcome valleys together and turned to me always with attention and care.

Most importantly, I own with special thanks to my parents, who implanted and supported my commitment and enthusiasm for research work. I am grateful for their inspiring guidance and continuous encouragement. I also express gratitude to one and all, who directly or indirectly have helped or currently helping me. 


\section{REFERENCES}

${ }^{1}$ Schmidt MJ, Mirnics K. Modeling interneuron dysfunction in schizophrenia. Dev Neurosci. 2012;34(2-3): 152-8.

${ }^{2}$ Hayashi-Takagi A, Vawter MP, Iwamoto K. Peripheral biomarkers revisited: integrative profiling of peripheral samples for psychiatric research. Biol Psychiatry. 2014; 75(12):920-8.

${ }^{3}$ Brennand KJ, Simone A, Tran N, Gage FH. Modeling psychiatric disorders at the cellular and network levels. Mol Psychiatry. 2012;17(12):1239-53.

${ }^{4}$ Selye H, Fortier C. Adaptive reactions to stress. Res Publ Assoc Res Nerv Ment Dis. 1949;29:3-18.

5 available at http://www.who.int/mediacentre/factsheets/fs369/en/.

${ }^{6}$ Kendler KS, Gardner CO, Neale MC, Prescott CA. Genetic risk factors for major depression in men and women: similar or different heritabilities and same or partly distinct genes. Psychol Med. 2001;31:605-616.

${ }^{7}$ Rucker JJ, McGuffin P. Chipping away at major depressive disorder. Genome Biology. 2014;15(7), 421.

${ }^{8}$ Major Depressive Disorder Working Group of the Psychiatric GWAS Consortium. A megaanalysis of genome-wide association studies for major depressive disorder. Molecular Psychiatry. 2013;18(4), 10.1038/mp.2012.21.

${ }^{9}$ Rucker JJ, McGuffin P. Polygenic heterogeneity: a complex model of genetic inheritance in psychiatric disorders. Biol Psychiatry. 2010; 68(4):312-3.

${ }^{10}$ Flint J, Kendler KS. The genetics of major depression. Neuron. 2014;81(3):484-503.

${ }^{11}$ Wermter AK, Laucht M, Schimmelmann BG, Banaschewski T, Sonuga-Barke EJ, Rietschel $\mathrm{M}$, Becker K. From nature versus nurture, via nature and nurture, to gene $\mathrm{x}$ environment interaction in mental disorders. Eur Child Adolesc Psychiatry. 2010;19(3):199-210.

${ }^{12}$ Pariante CM, Lightman SL. The HPA axis in major depression: classical theories and new developments. Trends Neurosci. 2008;31(9):464-8.

${ }^{13}$ Dowlati Y, Herrmann N, Swardfager W, Liu H, Sham L, Reim EK, Lanctôt KL. A metaanalysis of cytokines in major depression. Biol Psychiatry. 2010;67(5):446-57.

${ }^{14}$ Zorrilla EP, Luborsky L, McKay JR, Rosenthal R, Houldin A, Tax A, McCorkle R, Seligman DA, Schmidt K. The relationship of depression and stressors to immunological assays: a meta-analytic review. Brain Behav Immun. 2001;15(3):199-226.

${ }^{15}$ McIntyre RS, Soczynska JK, Konarski JZ, Woldeyohannes HO, Law CW, Miranda A, Fulgosi D, Kennedy SH. Should Depressive Syndromes Be Reclassified as "Metabolic Syndrome Type II"? Ann Clin Psychiatry. 2007;19(4):257-64.

${ }^{16}$ Hendrickx H, McEwen BS, Ouderaa F: Metabolism, mood and cognition in aging: the importance of lifestyle and dietary intervention. Neurobiol Aging 2005;26:1-5.

${ }^{17}$ Vangipuram M, Ting D, Kim S, Diaz R, Schüle B. Skin punch biopsy explant culture for derivation of primary human fibroblasts. J Vis Exp. 2013;77, e3779.

${ }^{18}$ Auburger G, Klinkenberg M, Drost J, Marcus K, Morales-Gordo B, Kunz WS, Brandt U, Broccoli V, Reichmann H, Gispert S, Jendrach M. Primary skin fibroblasts as a model of Parkinson's disease. Mol Neurobiol. 2012;46(1), 20-7.

${ }^{19}$ Bayreuther K, Francz PI, Gogol J, Hapke C, Maier M, Meinrath HG. Differentiation of primary and secondary fibroblasts in cell culture systems. Mutat Res. 1991; 256(2-6), 233-42.

${ }^{20}$ Hänzelmann S, Beier F, Gusmao EG, Koch CM, Hummel S, Charapitsa I, Joussen S, Benes V, Brümmendorf TH, Reid G, Costa IG, Wagner W. Replicative senescence associated with nuclear reorganization and with DNA methylation at specific transcription factor binding sites. Clin Epigenetics. 2015; 7(1), 19. 
${ }^{21}$ Manier DH, Shelton RC, Ellis TC, Peterson CS, Eiring A, Sulser F. Human fibroblasts as a relevant model to study signal transduction in affective disorders. J Affect Disord. 2000;61(12):51-8.

${ }^{22}$ Fournier M, Ferrari C, Baumann PS, Polari A, Monin A, Bellier-Teichmann T, Wulff J, Pappan KL, Cuenod M, Conus P, Do KQ. Impaired metabolic reactivity to oxidative stress in early psychosis patients. Schizophr Bull. 2014;40(5):973-83.

${ }^{23}$ Rieske P, Krynska B, Azizi SA. Human fibroblast-derived cell lines have characteristics of embryonic stem cells and cells of neuro-ectodermal origin. Differentiation. 2005; 73: 474483.

${ }^{24}$ Janmaat CJ, de Rooij KE, Locher H, de Groot SC, de Groot JC, Frijns JH, Huisman MA. Human Dermal Fibroblasts Demonstrate Positive Immunostaining for Neuron- and GliaSpecific Proteins. PLoS One. 2015;10(12):e0145235.

${ }^{25}$ Schmiedel JM, Klemm SL, Zheng Y, Sahay A, Blüthgen N, Marks DS, van Oudenaarden A. Gene expression. MicroRNA control of protein expression noise. Science. 2015;348(6230):128-32.

${ }^{26}$ Weber JA, Baxter DH, Zhang S, Huang DY, Huang KH, Lee MJ, Galas DJ, Wang K. The microRNA spectrum in 12 body fluids. Clin Chem. 2010;56(11):1733-41.

${ }^{27}$ Javidi MA, Ahmadi AH, Bakhshinejad B, Nouraee N, Babashah S, Sadeghizadeh M. Cellfree microRNAs as cancer biomarkers: the odyssey of miRNAs through body fluids. Med Oncol. 2014;31(12):295.

${ }^{28}$ Lin J, Li J, Huang B, Liu J, Chen X, Chen XM, Xu YM, Huang LF, Wang XZ. Exosomes: Novel Biomarkers for Clinical Diagnosis. ScientificWorldJournal. 2015;2015:657086.

${ }^{29}$ Valencia-Sanchez MA, Liu J, Hannon GJ, Parker R. Control of translation and mRNA degradation by miRNAs and siRNAs. Genes Dev. 2006;20:515-24.

${ }^{30}$ Pratt CA, Mowry KL. Taking a cellular road-trip: mRNA transport and anchoring. Curr Opin Cell Biol. 2013;25(1):99-106.

${ }^{31}$ Valinezhad Orang A, Safaralizadeh R, Kazemzadeh-Bavili M. Mechanisms of miRNAMediated Gene Regulation from Common Downregulation to mRNA-Specific Upregulation. Int J Genomics. 2014;2014:970607.

32 Bhattacharyya SN, Habermacher R, Martine U, Closs EI, Filipowicz W. Relief of microRNA-mediated translational repression in human cells subjected to stress. Cell.2006;125(6):1111-1124.

${ }^{33}$ Adjibade P, Mazroui R. Control of mRNA turnover: implication of cytoplasmic RNA granules. Semin Cell Dev Biol. 2014;34:15-23.

${ }^{34}$ Ziats MN, Rennert OM. Identification of differentially expressed microRNAs across the developing human brain. Mol Psychiatry. 2014;19(7):848-52.

${ }^{35}$ Shruti K, Shrey K, Vibha R. Micro RNAs: tiny sequences with enormous potential. Biochem Biophys Res Commun. 2011;407(3):445-9.

${ }^{36}$ Akerblom M, Jakobsson J. MicroRNAs as Neuronal Fate Determinants. Neuroscientist. 2013;20(3):235-242.

${ }^{37}$ Leung AK, Sharp PA. MicroRNA functions in stress responses. Mol Cell. 2010;40(2):20515 .

${ }^{38}$ Smalheiser NR. The RNA-centred view of the synapse: non-coding RNAs and synaptic plasticity. Philos Trans R Soc Lond B Biol Sci. 2014;369(1652).

${ }^{39}$ Mall C, Rocke DM, Durbin-Johnson B, Weiss RH. Stability of miRNA in human urine supports its biomarker potential. Biomark Med. 2013;7(4):623-31.

${ }^{40}$ Zhao H, Shen J, Hu Q, Davis W, Medico L, Wang D, Yan L, Guo Y, Liu B, Qin M,Nesline M, Zhu Q, Yao S, Ambrosone CB, Liu S. Effects of preanalytic variables on circulating microRNAs in whole blood. Cancer Epidemiol Biomarkers Prev. 2014;23(12):2643-8. 
${ }^{41}$ Fan HM, Sun XY, Guo W, Zhong AF, Niu W, Zhao L, Dai YH, Guo ZM, Zhang LY, Lu J. Differential expression of microRNA in peripheral blood mononuclear cells as specific biomarker for major depressive disorder patients. J Psychiatr Res. 2014;59:45-52.

${ }^{42}$ Serafini G, Pompili M, Innamorati M, Giordano G, Montebovi F, Sher L, Dwivedi Y, Girardi P. The role of microRNAs in synaptic plasticity, major affective disorders and suicidal behavior. Neurosci Res. 2012;73(3):179-90.

${ }^{43}$ Chen H, Wang N, Burmeister M, McInnis MG. MicroRNA expression changes in lymphoblastoid cell lines in response to lithium treatment. Int $\mathbf{J}$ Neuropsychopharmacol. 2009;12(7):975-81.

${ }^{44}$ Sun XY, Zhang J, Niu W, Guo W, Song HT, Li HY, Fan HM, Zhao L, Zhong AF, Dai YH, Guo ZM, Zhang LY, Lu J, Zhang QL. A preliminary analysis of microRNA as potential clinical biomarker for schizophrenia. Am J Med Genet B Neuropsychiatr Genet. 2015 Feb 5.

${ }^{45}$ Hommers LG, Domschke K, Deckert J. Heterogeneity and Individuality: microRNAs in Mental Disorders. J Neural Transm. 2014 Nov 14.

${ }^{46}$ Dwivedi Y. Emerging role of microRNAs in major depressive disorder: diagnosis and therapeutic implications. Dialogues Clin Neurosci. 2014;16(1):43-61.

${ }^{47}$ Maffioletti E, Tardito D, Gennarelli M, Bocchio-Chiavetto L. Micro spies from the brain to the periphery: new clues from studies on microRNAs in neuropsychiatric disorders. Front Cell Neurosci. 2014;8:75.

${ }^{48}$ Xu L, Qi X, Duan S, Xie Y, Ren X, Chen G, Yang X, Han L, Dong Q. MicroRNAs: potential biomarkers for disease diagnosis. Biomed Mater Eng. 2014;24(6):3917-25.

${ }^{49}$ Takahashi K, Yamanaka S. Induction of pluripotent stem cells from mouse embryonic and adult fibroblast cultures by defined factors. Cell. 2006;126(4):663-76.

${ }^{50}$ Brouwer M, Zhou H, Nadif Kasri N. Choices for induction of pluripotency: recent developments in human induced pluripotent stem cell reprogramming strategies. Stem Cell Rev. 2016;12(1):54-72.

${ }^{51}$ Deng XY, Wang H, Wang T, Fang XT, Zou LL, Li ZY, Liu CB. Non-viral methods for generating integration-free, induced pluripotent stem cells. Curr Stem Cell Res Ther. 2015;10(2):153-8.

${ }^{52}$ Yu J, Vodyanik MA, Smuga-Otto K, Antosiewicz-Bourget J, Frane JL, Tian S, Nie J, Jonsdottir GA, Ruotti V, Stewart R, Slukvin II, Thomson JA. Induced pluripotent stem cell lines derived from human somatic cells. Science. 2007;318(5858):1917-20.

${ }^{53}$ Muratore CR, Srikanth P, Callahan DG, Young-Pearse TL. Comparison and optimization of hiPSC forebrain cortical differentiation protocols. PLoS One. 2014;9(8):e105807.

${ }^{54}$ Mariani J, Simonini MV, Palejev D, Tomasini L, Coppola G, Szekely AM, Horvath TL, Vaccarino FM. Modeling human cortical development in vitro using induced pluripotent stem cells. Proc Natl Acad Sci U S A. 2012;109(31):12770-5.

${ }^{55}$ Ming GL, Song H. Adult neurogenesis in the mammalian brain: significant answers and significant questions. Neuron. 2011;70(4):687-702.

${ }^{56}$ Liu Y, Liu H, Sauvey C, Yao L, Zarnowska ED, Zhang SC. Directed differentiation of forebrain GABA interneurons from human pluripotent stem cells. Nat Protoc. 2013;8(9):1670-9.

${ }^{57}$ Maroof AM, Keros S, Tyson JA, Ying SW, Ganat YM, Merkle FT, Liu B, Goulburn A, Stanley EG, Elefanty AG, Widmer HR, Eggan K, Goldstein PA, Anderson SA, Studer L. Directed differentiation and functional maturation of cortical interneurons from human embryonic stem cells. Cell Stem Cell. 2013;12(5):559-72.

${ }^{58}$ Boyer LF, Campbell B, Larkin S, Mu Y, Gage FH. Dopaminergic differentiation of human pluripotent cells. Curr Protoc Stem Cell Biol. 2012;Chapter 1:Unit1H.6. 
${ }^{59}$ Sánchez-Danés A, Consiglio A, Richaud Y, Rodríguez-Pizà I, Dehay B, Edel M, Bové J, Memo M, Vila M, Raya A, Izpisua Belmonte JC. Efficient generation of A9 midbrain dopaminergic neurons by lentiviral delivery of LMX1A in human embryonic stem cells and induced pluripotent stem cells. Hum Gene Ther. 2012;23(1):56-69.

${ }^{60}$ Vazin T, Ball KA, Lu H, Park H, Ataeijannati Y, Head-Gordon T, Poo MM, Schaffer DV. Efficient derivation of cortical glutamatergic neurons from human pluripotent stem cells: a model system to study neurotoxicity in Alzheimer's disease. Neurobiol Dis. 2014;62:62-72.

${ }^{61}$ Gage FH. Mammalian neural stem cells. Science. 2000; 287(5457):1433-8.

${ }^{62}$ Zhao C, Teng EM, Summers RG Jr, Ming GL, Gage FH. Distinct morphological stages of dentate granule neuron maturation in the adult mouse hippocampus. J Neurosci. 2006;26(1):311 .

${ }^{63}$ Yu DX, Di Giorgio FP, Yao J, Marchetto MC, Brennand K, Wright R, Mei A, McHenry L, Lisuk D, Grasmick JM, Silberman P, Silberman G, Jappelli R, Gage FH. Modeling hippocampal neurogenesis using human pluripotent stem cells. Stem Cell Reports. 2014;2(3):295-310.

${ }^{64}$ Zhao C, Deng W, Gage FH. Mechanisms and functional implications of adult neurogenesis. Cell. 2008;132(4):645-60.

${ }^{65}$ Apple DM, Fonseca RS, Kokovay E. The role of adult neurogenesis in psychiatric and cognitive disorders. Brain Res. 2016; pii: S0006-8993(16)00043-3.

${ }^{66}$ Mahar I, Bambico FR, Mechawar N, Nobrega JN. Stress, serotonin, and hippocampal neurogenesis in relation to depression and antidepressant effects. Neurosci Biobehav Rev. 2014;38:173-92.

${ }^{67}$ Allen KM, Fung SJ, Shannon Weickert C. Cell proliferation is reduced in the hippocampus in schizophrenia. Aust N Z J Psychiatry. 2015; pii: 0004867415589793.

${ }^{68}$ Manier DH, Eiring A, Shelton RC, Sulser F. Beta-adrenoceptor-linked protein kinase A (PKA) activity in human fibroblasts from normal subjects and from patients with major depression. Neuropsychopharmacology. 1996;15(6):555-61.

${ }^{69}$ Akin D, Manier DH, Sanders-Bush E, Shelton RC. Decreased serotonin 5-HT2A receptorstimulated phosphoinositide signaling in fibroblasts from melancholic depressed patients. Neuropsychopharmacology. 2004;29(11):2081-7.

${ }^{70}$ Irizarry RA, Hobbs B, Collin F, et al. Exploration, normalization, and summaries of high density oligonucleotide array probe level data. Biostatistics. 2004;4(2):249-264.

${ }^{71}$ Xiaowei Wan. miRDB: a microRNA target prediction and functional annotation database with a wiki interface. RNA. 2008; 14(6):1012-1017

${ }^{72}$ Maragkakis M, Alexiou P, Papadopoulos GL, Reczko M, Dalamagas T, Giannopoulos G, Goumas G, Koukis E, Kourtis K, Simossis VA, Sethupathy P, Vergoulis T, Koziris N, Sellis T, Tsanakas P, Hatzigeorgiou AG. Accurate microRNA target prediction correlates with protein repression levels. BMC Bioinformatics. 2009;10:295.

73 Vlachos IS, Kostoulas N, Vergoulis T, Georgakilas G, Reczko M, Maragkakis M,Paraskevopoulou MD, Prionidis K, Dalamagas T, Hatzigeorgiou AG. DIANA miRPath v.2.0: investigating the combinatorial effect of microRNAs in pathways. Nucleic Acids Res. 2012 Jul;40(Web Server issue):W498-504.

${ }^{74}$ Basta-Kaim A, Budziszewska B, Jaworska-Feil L, Tetich M, Kubera M, Leśkiewicz M, Otczyk M, Lasoń W. Antipsychotic drugs inhibit the human corticotropin-releasing-hormone gene promoter activity in neuro-2A cells-an involvement of protein kinases. Neuropsychopharmacology. 2006;31(4):853-65.

${ }^{75}$ Mattson MP, Rychlik B, Cheng B. Degenerative and axon outgrowth-altering effects of phencyclidine in human fetal cerebral cortical cells. Neuropharmacology. 1992;31(3):279-91. 
${ }^{76}$ Puro DG. Cholinergic transmission by embryonic retinal neurons in culture: inhibition by dopamine. Brain Res. 1983;285(1):79-86.

${ }^{77}$ Ono T, Hashimoto E, Ukai W, Ishii T, Saito T. The role of neural stem cells for in vitro models of schizophrenia: neuroprotection via Akt/ERK signal regulation. Schizophr Res. 2010;122(1-3):239-47.

${ }^{78}$ Post A, Holsboer F, Behl C. Induction of NF-kappaB activity during haloperidol-induced oxidative toxicity in clonal hippocampal cells: suppression of NF-kappaB and neuroprotection by antioxidants. J Neurosci. 1998;18(20):8236-46.

${ }^{79}$ Valamehr B, Abujarour R, Robinson M, Le T, Robbins D, Shoemaker D, Flynn P. A novel platform to enable the high-throughput derivation and characterization of feeder-free human iPSCs. Sci Rep. 2012;2:213.

${ }^{80}$ Krzystek-Korpacka M, Diakowska D, Bania J, Gamian A. Expression stability of common housekeeping genes is differently affected by bowel inflammation and cancer: implications for finding suitable normalizers for inflammatory bowel disease studies. Inflamm Bowel Dis. 2014;20(7):1147-56.

${ }^{81}$ Kulkarni B, Mohammed I, Hopkinson A, Dua HS. Validation of endogenous control genes for gene expression studies on human ocular surface epithelium. PLoS One. 2011;6(8):e22301.

${ }^{82}$ Moran EP, Ma JX. Therapeutic Effects of PPAR $\alpha$ on Neuronal Death and Microvascular Impairment. PPAR Res. 2015;2015:595426.

${ }^{83}$ Fidaleo M, Fanelli F, Ceru MP, Moreno S. Neuroprotective properties of peroxisome proliferator-activated receptor alpha $(\mathrm{PPAR} \alpha)$ and its lipid ligands.Curr Med Chem. 2014;21(24):2803-21.

${ }^{84}$ Uyeda K, Repa JJ. Carbohydrate response element binding protein, ChREBP, a transcription factor coupling hepatic glucose utilization and lipid synthesis. Cell Metab. 2006;4(2):107-10.

${ }^{85}$ Pruitt K, Der CJ. Ras and Rho regulation of the cell cycle and oncogenesis. Cancer Lett. 2001;171(1):1-10.

${ }^{86}$ Bakthisaran R, Tangirala R, Rao CM. Small heat shock proteins: Role in cellular functions and pathology. Biochim Biophys Acta. 2015;1854(4):291-319.

${ }^{87}$ Ishihara N, Takagi N, Niimura M, Takagi K, Nakano M, Tanonaka K, Funakoshi H, Matsumoto K, Nakamura T, Takeo S. Inhibition of apoptosis-inducing factor translocation is involved in protective effects of hepatocyte growth factor against excitotoxic cell death in cultured hippocampal neurons. J Neurochem. 2005;95(5):1277-86.

${ }^{88}$ Forte G, Minieri M, Cossa P, Antenucci D, Sala M, Gnocchi V, Fiaccavento R,Carotenuto F, De Vito P, Baldini PM, Prat M, Di Nardo P. Hepatocyte growth factor effects on mesenchymal stem cells: proliferation, migration, and differentiation. Stem Cells. 2006;24(1):23-33.

${ }^{89}$ Higashio K, Shima N. Tumor cytotoxic activity of HGF-SF. EXS. 1993;65:351-68.

${ }^{90}$ Kim WH, Matsumoto K, Bessho K, Nakamura T. Growth inhibition and apoptosis in liver myofibroblasts promoted by hepatocyte growth factor leads to resolution from liver cirrhosis. Am J Pathol. 2005;166(4):1017-28.

${ }^{91}$ Tulasne D, Foveau B. The shadow of death on the MET tyrosine kinase receptor. Cell Death Differ. 2008;15(3):427-34.

${ }^{92}$ Arnold SE, Xie SX, Leung YY, Wang LS, Kling MA, Han X, Kim EJ, Wolk DA, Bennett DA, Chen-Plotkin A, Grossman M, Hu W, Lee VM, Mackin RS, Trojanowski JQ, Wilson RS, Shaw LM. Plasma biomarkers of depressive symptoms in older adults. Transl Psychiatry. 2012;2:e65. 
${ }^{93}$ Arakaki N, Kajihara T, Arakaki R, Ohnishi T, Kazi JA, Nakashima H, Daikuhara Y. Involvement of oxidative stress in tumor cytotoxic activity of hepatocyte growth factor/scatter factor. J Biol Chem. 1999;274(19):13541-6.

${ }^{94}$ Hatziapostolou M, Polytarchou C, Iliopoulos D. miRNAs link metabolic reprogramming to oncogenesis. Trends Endocrinol Metab. 2013;24(7):361-73.

${ }^{95}$ Suh SS, Yoo JY, Nuovo GJ, Jeon YJ, Kim S, Lee TJ, Kim T, Bakàcs A, Alder H,Kaur B, Aqeilan RI, Pichiorri F, Croce CM. MicroRNAs/TP53 feedback circuitry in glioblastoma multiforme. Proc Natl Acad Sci U S A. 2012;109(14):5316-21.

${ }^{96}$ Zhang N, Li X, Wu CW, Dong Y, Cai M, Mok MT, Wang H, Chen J, Ng SS, Chen M, Sung JJ, Yu J. microRNA-7 is a novel inhibitor of YY1 contributing to colorectal tumorigenesis. Oncogene. 2013;32(42):5078-88.

${ }^{97}$ Henderson JK, Draper JS, Baillie HS, Fishel S, Thomson JA, Moore H, Andrews PW. Preimplantation human embryos and embryonic stem cells show comparable expression of stage-specific embryonic antigens. Stem Cells. 2002;20(4):329-37.

${ }^{98}$ Chambers I, Tomlinson SR. The transcriptional foundation of pluripotency. Development. 2009;136(14):2311-22.

${ }^{99}$ Othman M, Lu C, Klueber K, Winstead W, Roisen F. Clonal analysis of adult human olfactory neurosphere forming cells. Biotech Histochem. 2005 Sep-Dec;80(5-6):189-200.

${ }^{100}$ Iwano T, Masuda A, Kiyonari H, Enomoto H, Matsuzaki F. Prox1 postmitotically defines dentate gyrus cells by specifying granule cell identity over CA3 pyramidal cell fate in the hippocampus. Development. 2012;139(16):3051-62.

${ }^{101}$ Lavado A, Lagutin OV, Chow LM, Baker SJ, Oliver G. Prox1 is required for granule cell maturation and intermediate progenitor maintenance during brain neurogenesis. PLoS Biol. 2010;8(8). pii: e1000460.

${ }^{102}$ Bahn S, Chan MK. What can we learn about depression from gene expression in peripheral tissues? Biol Psychiatry. 2015;77(3):207-9

${ }^{103}$ Rehman SU, Shah SA, Ali T, Chung JI, Kim MO. Anthocyanins Reversed D-GalactoseInduced Oxidative Stress and Neuroinflammation Mediated Cognitive Impairment in Adult Rats. Mol Neurobiol. 2016 Jan 6.

${ }^{104}$ Marroquin LD, Hynes J, Dykens JA, Jamieson JD, Will Y. Circumventing the Crabtree effect: replacing media glucose with galactose increases susceptibility of HepG2 cells to mitochondrial toxicants. Toxicol Sci. 2007; 97: 539-547.

${ }^{105}$ Gibson SA, Korade Z, Shelton RC. Oxidative stress and glutathione response in tissue cultures from persons with major depression. J Psychiatr Res. 2012; 46:1326-1332.

${ }^{106}$ Jacobs RA, Sly WS, Majerus PW. The regulation of fatty acid biosynthesis in human skin fibroblasts. J Biol Chem 1973;248: 1268-1276.

${ }^{107}$ Pike MC, Snyderman R. Lipid requirements for leukocyte chemotaxis and phagocytosis: effects of inhibitors of phospholipid and cholesterol synthesis. J Immunol. 1980;124(4):19639 .

${ }^{108}$ Santos CR, Schulze A. Lipid metabolism in cancer. FEBS J. 2012 Aug;279(15):2610-23.

${ }^{109}$ Jacobs RA, Sly WS, Majerus PW. The regulation of fattyacid biosynthesis in human skin fibroblasts. J. Biol. Chem. 1973; 248:1268-1276.

${ }^{110}$ Raff RA: Induction of fatty acid synthesis in cultured mammalian cells: effects of cycloheximide and X-rays. J Cell Physiol.1970; 75: 341-351.

${ }^{111}$ I. Bensaude,J.Callahan,M.Philippart,Fabry'sdiseaseasan -galactosidosis: evidence for anconfiguration intrihexosyl ceramide, Biochem.Biophys.Res.Commun.1971; 43:913-918.

112 Tsai KW, Wu CW, Hu LY, Li SC, Liao YL, et al. Epigenetic regulation of miR-34b and miR-129 expression in gastric cancer. Int J Cancer. 2011;129:2600-2610. 
${ }^{113}$ Lopresti AL, Maker GL, Hood SD, Drummond PD. A review of peripheral biomarkers in major depression: The potential of inflammatory and oxidative stress biomarkers. Prog Neuropsychopharmacol Biol Psychiatry. 2013;48C:102-111.

${ }^{114}$ Slavich GM, Irwin MR. From stress to inflammation and major depressive disorder: a social signal transduction theory of depression. Psychol Bull. 2014;140(3):774-815.

${ }^{115}$ Arias B, Fabbri C, Serretti A, Drago A, Mitjans M, Gastó C, Catalán R, Fañanás L. DISC1-TSNAX and DAOA genes in major depression and citalopram efficacy. J Affect Disord. 2014;168:91-7.

${ }^{116}$ Savitz J, Frank MB, Victor T, Bebak M, Marino JH, Bellgowan PS, McKinney BA, Bodurka J, Kent Teague T, Drevets WC. Inflammation and neurological disease-related genes are differentially expressed in depressed patients with mood disorders and correlate with morphometric and functional imaging abnormalities. Brain Behav Immun. 2013;31:161-71.

${ }^{117}$ Stringer JM, Barrand S, Western P. Fine-tuning evolution: germ-line epigenetics and inheritance. Reproduction. 2013;146(1):R37-48.

${ }^{118}$ Hoffmann A, Spengler D. DNA memories of early social life. Neuroscience. 2014;264:6475.

${ }^{119}$ Wright JW, Harding JW. The Brain Hepatocyte Growth Factor/c-Met Receptor System: A New Target for the Treatment of Alzheimer's Disease. J Alzheimers Dis. 2015;45(4):9851000 .

${ }^{120}$ Maina F, Klein R. Hepatocyte growth factor, a versatile signal for developing neurons. Nat Neurosci. 1999;2:213-217.

${ }^{121}$ Date I, Takagi N, Takagi K, Kago T, Matsumoto K, Nakamura T, Takeo S. Hepatocyte growth factor improved learning and memory dysfunction of microsphere-embolized rats. $\mathrm{J}$ Neurosci Res. 2004;78(3):442-53.

${ }^{122}$ Wakatsuki M, Akiyoshi J, Ichioka S, Tanaka Y, Tsuru J, Matsushita H, Hanada H, Isogawa K. Administration of antisense DNA for hepatocyte growth factor causes an depressive and anxiogenic response in rats. Neuropeptides. 2007;41(6):477-83.

${ }^{123}$ Arnold SE, Xie SX, Leung YY, Wang LS, Kling MA, Han X, Kim EJ, Wolk DA, Bennett DA, Chen-Plotkin A, Grossman M, Hu W, Lee VM, Mackin RS, Trojanowski JQ, Wilson RS, Shaw LM. Plasma biomarkers of depressive symptoms in older adults. Transl Psychiatry. 2012;2:e65.

${ }^{124}$ Dwivedi Y. Pathogenetic and therapeutic applications of microRNAs in major depressive disorder. Prog Neuropsychopharmacol Biol Psychiatry. 2016;64:341-8.

${ }^{125}$ Serafini G, Pompili M, Hansen KF, Obrietan K, Dwivedi Y, Shomron N, Girardi P. The involvement of microRNAs in major depression, suicidal behavior, and related disorders: a focus on miR-185 and miR-491-3p. Cell Mol Neurobiol. 2014;34(1):17-30.

${ }^{126}$ Li YJ, Xu M, Gao ZH, Wang YQ, Yue Z, Zhang YX, Li XX, Zhang C, Xie SY, Wang PY. Alterations of serum levels of BDNF-related miRNAs in patients with depression. PLoS One. 2013;8(5):e63648.

${ }^{127}$ Muinos-Gimeno M, Espinosa-Parrilla Y, Guidi M, Kagerbauer B, Sipila T, Maron E, et al. Human microRNAs miR-22, miR-138-2, miR-148a, and miR-488 are associated with panic disorder and regulate several anxiety candidate genes and related pathways. Biol Psychiatry. 2011;69:526-533.

${ }^{128}$ Vo N, Klein ME, Varlamova O, Keller DM, Yamamoto T, Goodman RH, et al. A cAMPresponse element binding protein-induced microRNA regulates neuronal morphogenesis. Proc Natl Acad Sci U S A. 2005;102:16426-16431.

${ }^{129}$ Belzeaux R, Bergon A, Jeanjean V, Loriod B, Formisano-Tréziny C, Verrier L, Loundou A, Baumstarck-Barrau K, Boyer L, Gall V, Gabert J, Nguyen C, Azorin JM, Naudin J, 
Ibrahim EC. Responder and nonresponder patients exhibit different peripheral transcriptional signatures during major depressive episode. Transl Psychiatry. 2012;2:e185.

${ }^{130}$ Kim AH, Reimers M, Maher B, Williamson V, McMichael O, McClay JL, et al. MicroRNA expression profiling in the prefrontal cortex of individuals affected with schizophrenia and bipolar disorders. Schizophr Res. 2010;124:183-191.

${ }^{131}$ Maussion G, Yang J, Yerko V, Barker P, Mechawar N, Ernst C, Turecki G. Regulation of a truncated form of tropomyosin-related kinase B (TrkB) by Hsa-miR-185* in frontal cortex of suicide completers. PLoS One. 2012;7(6):e39301.

${ }^{132}$ Guo AY, Sun J, Jia P, Zhao Z. A novel microRNA and transcription factor mediated regulatory network in schizophrenia. BMC Syst Biol. 2010;4:10.

${ }^{133}$ Smalheiser NR, Lugli G, Rizavi HS, Zhang H, Torvik VI, Pandey GN, et al. MicroRNA expression in rat brain exposed to repeated inescapable shock: differential alterations in learned helplessness vs. non-learned helplessness. Int $\mathbf{J}$ Neuropsychopharmacol. 2011;14:1315-1325.

${ }^{134}$ Wang WX, Rajeev BW, Stromberg AJ, Ren N, Tang G, Huang Q, et al. The expression of microRNA miR-107 decreases early in Alzheimer's disease and may accelerate disease progression through regulation of beta-site amyloid precursor protein-cleaving enzyme 1 . $J$ Neurosci. 2008;28:1213-1223.

${ }^{135}$ Bocchio-Chiavetto L, Maffioletti E, Bettinsoli P, Giovannini C, Bignotti S, Tardito D, Corrada D, Milanesi L, Gennarelli M. Blood microRNA changes in depressed patients during antidepressant treatment. Eur Neuropsychopharmacol. 2013;23(7):602-11.

${ }^{136}$ Fan HM, Sun XY, Guo W, Zhong AF, Niu W, Zhao L, Dai YH, Guo ZM, Zhang LY, Lu J. Differential expression of microRNA in peripheral blood mononuclear cells as specific biomarker for major depressive disorder patients. J Psychiatr Res. 2014;59:45-52.

${ }^{137}$ Zhang D, Wang Y, Shi Z, Liu J, Sun P, Hou X, Zhang J, Zhao S, Zhou BP, Mi J. Metabolic reprogramming of cancer-associated fibroblasts by IDH3 $\alpha$ downregulation. Cell Rep. 2015;10(8):1335-48.

${ }^{138}$ Nie S, Li K, Huang Y, Hu Q, Gao X, Jie S. miR-495 mediates metabolic shift in glioma cells via targeting Glut1. J Craniofac Surg. 2015;26(2):e155-8.

${ }^{139}$ Liu JJ, Green P, John Mann J, Rapoport SI, Sublette ME. Pathways of polyunsaturated fatty acid utilization: Implications for brain function in neuropsychiatric health and disease. Brain Res. 2015;1597:220-46.

${ }^{140}$ Nallamshetty S, Chan SY, Loscalzo J. Hypoxia: a master regulator of microRNA biogenesis and activity. Free Radic Biol Med. 2013;64:20-30.

${ }^{141}$ Cottrill KA, Chan SY, Loscalzo J. Hypoxamirs and Mitochondrial Metabolism. Antioxid Redox Signal. 2014;21(8):1189-201.

${ }^{142}$ Gottschalk MG, Wesseling H, Guest PC, Bahn S. Proteomic enrichment analysis of psychotic and affective disorders reveals common signatures in presynaptic glutamatergic signaling and energy metabolism. Int J Neuropsychopharmacol. $2014 ; 18(2)$.

${ }^{143}$ Liu CR, Miao J, Zhang YL, Liu YM, Yu BG. Effects of hypothyroidism on expression of CRMP2B and ARPC5 during development of the rat frontal cortex. Int $\mathrm{J}$ Biol Sci. 2013;9:209-218.

${ }^{144}$ Kobayashi H, Fukuda M. Rab35 regulates Arf6 activity through centaurin- $\beta 2$ (ACAP2) during neurite outgrowth. J Cell Sci. 2012;125(Pt 9):2235-43.

${ }^{145}$ Kähler AK, Djurovic S, Rimol LM, Brown AA, Athanasiu L, Jönsson EG, Hansen T, Gústafsson O, Hall H, Giegling I, Muglia P, Cichon S, Rietschel M, Pietiläinen OP, Peltonen L, Bramon E, Collier D, St Clair D, Sigurdsson E, Petursson H, Rujescu D, Melle I, Werge T, Steen VM, Dale AM, Matthews RT, Agartz I, Andreassen OA. Candidate gene analysis of the human natural killer-1 carbohydrate pathway and perineuronal nets in schizophrenia: 
B3GAT2 is associated with disease risk and cortical surface area. Biol Psychiatry. 2011;69(1):90-6.

${ }^{146}$ Liu J, Githinji J, McLaughlin B, Wilczek K, Nolta J. Role of miRNAs in neuronal differentiation from human embryonic stem cell-derived neural stem cells. Stem Cell Rev. 2012;8:1129-1137.

${ }^{147}$ Li B, Sun H. MiR-26a promotes neurite outgrowth by repressing PTEN expression. Mol Med Rep. 2013;8:676-680.

${ }^{148}$ Agostini M, Tucci P, Steinert JR, Shalom-Feuerstein R, Rouleau M, Aberdam D, et al. microRNA-34a regulates neurite outgrowth, spinal morphology, and function. Proc Natl Acad Sci USA. 2011;108:21099-21104.

149 Khudayberdiev S, Fiore R, Schratt G. MicroRNA as modulators of neuronal responses. Commun Integr Biol. 2009;2:411-413.

${ }^{150}$ Numakawa T, Richards M, Adachi N, Kishi S, Kunugi H, Hashido K. MicroRNA function and neurotrophin BDNF.Neurochem Int. 2011;59:551-558.

${ }^{151}$ Rodriguez-Aznar E, Barrallo-Gimeno A, Nieto MA. Scratch2 prevents cell cycle re-entry by repressing miR-25 in postmitotic primary neurons. J Neurosci. 2009;33:5095-5105.

${ }^{152}$ Aranha MM, Santos DM, Sola S, Steer CJ, Rodrigues CM. miR-34a regulates mouse neural stem cell differentiation. PLoS One.2011;6:e21396.

${ }^{153}$ Yadav S, Pandey A, Shukla A, Talwelkar SS, Kumar A, Pant AB, et al. miR-497 and miR$302 \mathrm{~b}$ regulate ethanol-induced neuronal cell death through BCL2 protein and cyclin D2. J Biol Chem.2011;286:37347-37357.

${ }^{154}$ Jovicic A, Roshan R, Moisoi N, Pradervand S, Moser R, Pillai B, et al. Comprehensive expression analyses of neural cell-type-specific miRNAs identify new determinants of the specification and maintenance of neuronal phenotypes. J Neurosci.2013;33:5127-5137.

${ }^{155}$ Qi L, Hongjuan H, Ning G, Zhengbin H, Yanjiang X, Tiebo Z, et al. miR-370 is stagespecifically expressed during mouse embryonic development and regulates Dnmt3a. FEBS Lett.2013;587:775-781.

${ }^{156}$ Lin ST, Huang Y, Zhang L, Heng MY, Ptacek LJ, Fu YH. MicroRNA-23a promotes myelination in the central nervous system. Proc Natl Acad Sci USA. 2013;110:17468-17473.

${ }^{157}$ Miller BH, Zeier Z, Xi L, Lanz TA, Deng S, Strathmann J, et al. MicroRNA-132 dysregulation in schizophrenia has implications for both neurodevelopment and adult brain function. Proc Natl Acad Sci USA. 2012;109:3125-3130.

${ }^{158}$ Kumar P, Dezso Z, MacKenzie C, Oestreicher J, Agoulnik S, Byrne, et al. Circulating miRNA biomarkers for Alzheimer's disease. PLoS One. 2013;8:e69807.

${ }^{159}$ Gaughwin PM, Ciesla M, Lahiri N, Tabrizi SJ, Brundin P, Bjorkqvist M. Hsa-miR-34b is a plasma-stable microRNA that is elevated in pre-manifest Huntington's disease. Hum Mol Genet. 2011;20:2225-2237.

${ }^{160}$ Minones-Moyano E, Porta S, Escaramis G, Rabionet R, Iraola S, Kagerbauer B, et al. MicroRNA profiling of Parkinson's disease brains identifies early downregulation of miR34b/c which modulate mitochondrial function. Hum Mol Genet.2011;20:3067-3078.

${ }^{161}$ Zhang R, Zhang Q, Niu J, Lu K, Xie B, Cui D, et al. Screening of microRNAs associated with Alzheimer's disease using oxidative stress cell model and different strains of senescence accelerated mice. J Neurol Sci. 2014;338:57-64.

162 Truettner JS, Katyshev V, Esen-Bilgin N, Dietrich WD, Dore-Duffy P. Hypoxia alters MicroRNA expression in rat cortical pericytes. Microrna. 2013;2:32-45.

${ }^{163}$ Zucchi FC, Yao Y, Ward ID, Inytskyy Y, Olson DM, Benzies K, et al. Maternal stress induces epigenetic signatures of psychiatric and neurological diseases in the offspring. PLoS One. 2013;8:e56967. 
${ }^{164}$ Waldera-Lupa DM, Kalfalah F, Florea AM, Sass S, Kruse F, Rieder V, Tigges J, Fritsche E, Krutmann J, Busch H, Boerries M, Meyer HE, Boege F, Theis F, Reifenberger G, Stühler K. Proteome-wide analysis reveals an age-associated cellular phenotype of in situ aged human fibroblasts. Aging (Albany NY). 2014;6(10):856-78.

${ }^{165}$ Koch CM, Suschek CV, Lin Q, Bork S, Goergens M, Joussen S, Pallua N, Ho AD, Zenke M, Wagner W. Specific age-associated DNA methylation changes in human dermal fibroblasts. PLoS One. 2011;6(2):e16679.

${ }^{166}$ Peterson C, Goldman JE. Alterations in calcium content and biochemical processes in cultured skin fibroblasts from aged and Alzheimer donors. Proc Natl Acad Sci U S A. 1986;83(8):2758-62.

${ }^{167}$ Sprenger A, Küttner V, Biniossek ML, Gretzmeier C, Boerries M, Mack C, Has C, Bruckner-Tuderman L, Dengjel J. Comparative quantitation of proteome alterations induced by aging or immortalization in primary human fibroblasts and keratinocytes for clinical applications. Mol Biosyst. 2010;6(9):1579-82.

${ }^{168}$ Antequera F, Boyes J, Bird A. High levels of de novo methylation and altered chromatin structure at CpG islands in cell lines. Cell. 1990;62(3):503-14.

${ }^{169}$ Nestor CE, Ottaviano R, Reinhardt D, Cruickshanks HA, Mjoseng HK, McPherson RC, Lentini A, Thomson JP, Dunican DS, Pennings S, Anderton SM, Benson M, Meehan RR. Rapid reprogramming of epigenetic and transcriptional profiles in mammalian culture systems. Genome Biol. 2015;16:11

${ }^{170}$ Lundgaard I, Osório MJ, Kress BT, Sanggaard S, Nedergaard M. White matter astrocytes in health and disease. Neuroscience. 2014;276:161-73.

${ }^{171}$ Glaros T, Larsen M, Li L. Macrophages and fibroblasts during inflammation, tissue damage and organ injury. Front Biosci (Landmark Ed). 2009;14:3988-93.

${ }^{172}$ Nelson PT, Kiriakidou M, Mourelatos Z, Tan GS, Jennings MH, Xie K, Wang WX. Highthroughput experimental studies to identify miRNA targets directly, with special focus on the mammalian brain. Brain Res. 2010;1338:122-30.

${ }^{173}$ Mertens J, Wang QW, Kim Y, Yu DX, Pham S, Yang B, Zheng Y, Diffenderfer KE, Zhang J, Soltani S, Eames T, Schafer ST, Boyer L, Marchetto MC, Nurnberger JI, Calabrese JR, Ødegaard KJ, McCarthy MJ, Zandi PP, Alda M, Nievergelt CM; Pharmacogenomics of Bipolar Disorder Study, Mi S, Brennand KJ, Kelsoe JR, Gage FH, Yao J. Differential responses to lithium in hyperexcitable neurons from patients with bipolar disorder. Nature. 2015;527(7576):95-9.

${ }^{174}$ Darcet F, Gardier AM, Gaillard R, David DJ, Guilloux JP. Cognitive Dysfunction in Major Depressive Disorder. A Translational Review in Animal Models of the Disease. Pharmaceuticals (Basel). 2016;9(1). pii: E9.

175 Brunton, L; Chabner, B; Knollman, B (2010). Goodman and Gilman's The Pharmacological Basis of Therapeutics (12th ed.). New York: McGraw-Hill Professional.

${ }^{176}$ Schmitt A, Zink M, Müller B, May B, Herb A, Jatzko A, Braus DF, Henn FA. Effects of long-term antipsychotic treatment on NMDA receptor binding and gene expression of subunits. Neurochem Res. 2003;28(2):235-41.

${ }^{177}$ Schmitt A, Zink M, Petroianu G, May B, Braus DF, Henn FA. Decreased gene expression of glial and neuronal glutamate transporters after chronic antipsychotic treatment in rat brain. Neurosci Lett. 2003;347(2):81-4.

${ }^{178}$ Leysen JE, Janssen PM, Megens AA, Schotte A. Risperidone: a novel antipsychotic with balanced serotonin-dopamine antagonism, receptor occupancy profile, and pharmacologic activity. J Clin Psychiatry. 1994;55 Suppl:5-12.

${ }^{179}$ Lieberman JA, Tollefson GD, Charles C, Zipursky R, Sharma T, Kahn RS, Keefe RS, Green AI, Gur RE, McEvoy J, Perkins D, Hamer RM, Gu H, Tohen M; HGDH Study Group. 
Antipsychotic drug effects on brain morphology in first-episode psychosis. Arch Gen Psychiatry. 2005;62(4):361-70.

${ }^{180}$ Ito K, Nakazawa K, Koizumi S, Liu M, Takeuchi K, Hashimoto T, Ohno Y, Inoue K. Inhibition by antipsychotic drugs of L-type $\mathrm{Ca} 2+$ channel current in PC12 cells. Eur J Pharmacol. 1996;314(1-2):143-50.

${ }^{181}$ Santi CM, Cayabyab FS, Sutton KG, McRory JE, Mezeyova J, Hamming KS, Parker D, Stea A, Snutch TP. Differential inhibition of T-type calcium channels by neuroleptics. J Neurosci. 2002;22(2):396-403.

${ }^{182}$ Miyata T, Maeda T, Lee JE. NeuroD is required for differentiation of the granule cells in the cerebellum and hippocampus. Genes Dev. 1999;13(13):1647-52.

${ }^{183}$ Schwab MH, Bartholomae A, Heimrich B, Feldmeyer D, Druffel-Augustin S, Goebbels S, Naya FJ, Zhao S, Frotscher M, Tsai MJ, Nave KA. Neuronal basic helix-loop-helix proteins (NEX and BETA2/Neuro D) regulate terminal granule cell differentiation in the hippocampus. J Neurosci. 2000;20(10):3714-24.

${ }^{184}$ Page RL, Ambady S, Holmes WF, Vilner L, Kole D, Kashpur O, Huntress V, Vojtic I, Whitton $\mathrm{H}$, Dominko T. Induction of stem cell gene expression in adult human fibroblasts without transgenes. Cloning Stem Cells. 2009;11:417-26.

${ }^{185}$ Hsieh J, Nakashima K, Kuwabara T, Mejia E, Gage FH. Histone deacetylase inhibitionmediated neuronal differentiation of multipotent adult neural progenitor cells. Proc Natl Acad Sci U S A. 2004;101(47):16659-64.

186 Tonge PD, Andrews PW. Retinoic acid directs neuronal differentiation of human pluripotent stem cell lines in a non-cell-autonomous manner. Differentiation. 2010;80(1):2030.

${ }^{187}$ Toro CT, Deakin JF. Adult neurogenesis and schizophrenia: a window on abnormal early brain development? Schizophr Res. 2007;90(1-3):1-14.

188 Newton SS, Duman RS. Neurogenic actions of atypical antipsychotic drugs and therapeutic implications. CNS Drugs. 2007;21(9):715-25.

${ }^{189}$ Kodama M, Fujioka T, Duman RS. Chronic olanzapine or fluoxetine administration increases cell proliferation in hippocampus and prefrontal cortex of adult rat. Biol Psychiatry. 2004;56(8):570-80.

${ }^{190}$ Nowakowska E, Kus K, Ratajczak P, Cichocki M, Woźniak A. The influence of aripiprazole, olanzapine and enriched environment on depressant-like behavior, spatial memory dysfunction and hippocampal level of BDNF in prenatally stressed rats. Pharmacol Rep. 2014;66(3):404-11.

${ }^{191}$ Csernansky JG, Martin MV, Czeisler B, Meltzer MA, Ali Z, Dong H. Neuroprotective effects of olanzapine in a rat model of neurodevelopmental injury. Pharmacol Biochem Behav. 2006;83(2):208-13.

192 Keilhoff G, Grecksch G, Bernstein HG, Roskoden T, Becker A. Risperidone and haloperidol promote survival of stem cells in the rat hippocampus. Eur Arch Psychiatry Clin Neurosci. 2010;260(2):151-62.

${ }^{193}$ Behl C, Lezoulac'h F, Widmann M, Rupprecht R, Holsboer F. Oxidative stress-resistant cells are protected against haloperidol toxicity. Brain Res. 1996;717(1-2):193-5.

${ }^{194}$ Fehér LZ, Kálmán J, Puskás LG, Gyülvészi G, Kitajka K, Penke B, Palotás M, Samarova EI, Molnár J, Zvara A, Matin K, Bódi N, Hugyecz M, Pákáski M, Bjelik A, Juhász A, Bogáts $\mathrm{G}$, Janka Z, Palotás A. Impact of haloperidol and risperidone on gene expression profile in the rat cortex. Neurochem Int. 2005;47(4):271-80.

195 Pillai A, Parikh V, Terry AV Jr, Mahadik SP. Long-term antipsychotic treatments and crossover studies in rats: differential effects of typical and atypical agents on the expression of 
antioxidant enzymes and membrane lipid peroxidation in rat brain. J Psychiatr Res. 2007 Aug;41(5):372-86.

196 Pillai A, Terry AV Jr, Mahadik SP. Differential effects of long-term treatment with typical and atypical antipsychotics on NGF and BDNF levels in rat striatum and hippocampus. Schizophr Res. 2006;82(1):95-106.

${ }^{197}$ Parikh V, Khan MM, Mahadik SP. Olanzapine counteracts reduction of brain-derived neurotrophic factor and TrkB receptors in rat hippocampus produced by haloperidol. Neurosci Lett. 2004;356(2):135-9.

${ }^{198}$ Pillai A, Mahadik SP. Differential effects of haloperidol and olanzapine on the expression of erythropoietin and its receptor in rat hippocampus and striatum. $J$ Neurochem. 2006;98(5):1411-22.

${ }^{199}$ Green W, Patil P, Marsden CA, Bennett GW, Wigmore PM. Treatment with

olanzapine increases cell proliferation in the subventricular zone and prefrontal cortex. Brain Res. 2006;1070(1):242-5.

${ }^{200}$ Jun H, Mohammed Qasim Hussaini S, Rigby MJ, Jang MH. Functional role of adult hippocampal neurogenesis as a therapeutic strategy for mental disorders. Neural Plast. 2012;2012:854285.

${ }^{201}$ Kurihara, Y., Suzuki, T., Sakaue, M., Murayama, O., Miyazaki, Y., Onuki, A., Aoki, T., Saito, M., Fujii, Y., Hisasue, M., Tanaka, K., and Takizawa, T. Valproic acid, a histone deacetylase inhibitor, decreases proliferation of and induces specific neurogenic differentiation of canine adipose tissue-derived stem cells. J. Vet. Med. Sci. 2014;74: 15-23.

${ }^{202}$ Johnson GV, Jope RS. The role of microtubule-associated protein 2 (MAP-2) in neuronal growth, plasticity, and degeneration. J Neurosci Res. 1992;33(4):505-12.

${ }^{203}$ Sánchez C, Díaz-Nido J, Avila J. Phosphorylation of microtubule-associated protein 2 (MAP2) and its relevance for the regulation of the neuronal cytoskeleton function. Prog Neurobiol. 2000;61(2):133-68.

${ }^{204}$ Law AJ, Hutchinson LJ, Burnet PW, Harrison PJ. Antipsychotics increase microtubuleassociated protein 2 mRNA but not spinophilin mRNA in rat hippocampus and cortex. $\mathrm{J}$ Neurosci Res. 2004;76(3):376-82.

${ }^{205}$ Konradi C, Heckers S. Antipsychotic drugs and neuroplasticity: insights into the treatment and neurobiology of schizophrenia. Biol Psychiatry. 2001;50(10):729-42.

${ }^{206}$ Lidow MS, Song ZM, Castner SA, Allen PB, Greengard P, Goldman-Rakic PS. Antipsychotic treatment induces alterations in dendrite- and spine-associated proteins in dopamine-rich areas of the primate cerebral cortex. Biol Psychiatry. 2001;49(1):1-12.

207 Critchlow HM, Maycox PR, Skepper JN, Krylova O. Clozapine and haloperidol differentially regulate dendritic spine formation and synaptogenesis in rat hippocampal neurons. Mol Cell Neurosci. 2006;32(4):356-65.

${ }^{208}$ Arnold SE, Lee VM, Gur RE, Trojanowski JQ. Abnormal expression of two microtubuleassociated proteins (MAP2 and MAP5) in specific subfields of the hippocampal formation in schizophrenia. Proc Natl Acad Sci U S A. 1991;88(23):10850-4.

${ }^{209}$ Cotter D, Kerwin R, Doshi B, Martin CS, Everall IP. Alterations in hippocampal nonphosphorylated MAP2 protein expression in schizophrenia. Brain Res. 1997;765(2):238-46.

${ }^{210}$ Cotter D, Wilson S, Roberts E, Kerwin R, Everall IP. Increased dendritic MAP2 expression in the hippocampus in schizophrenia. Schizophr Res. 2000;41(2):313-23.

${ }^{211}$ Eng LF, Vanderhaeghen JJ, Bignami A \& Gerste B (1971). An acidic protein isolated from fibrous astrocytes. Brain Research. 1971;28: 351-354.

${ }^{212}$ Feresten AH, Barakauskas V, Ypsilanti A, Barr AM, Beasley CL. Increased expression of glial fibrillary acidic protein in prefrontal cortex in psychotic illness. Schizophr Res. 2013;150(1):252-7. 
${ }^{213}$ Belinsky GS, Rich MT, Sirois CL, Short SM, Pedrosa E, Lachman HM, Antic SD. Patchclamp recordings and calcium imaging followed by single-cell PCR reveal the developmental profile of 13 genes in iPSC-derived human neurons. Stem Cell Res. 2014;12(1):101-18.

${ }^{214}$ Farra N, Zhang WB, Pasceri P, Eubanks JH, Salter MW, Ellis J. Rett syndrome induced pluripotent stem cell-derived neurons reveal novel neurophysiological alterations. Mol Psychiatry. 2012;17(12):1261-71.

${ }^{215}$ Handel AE, Chintawar S, Lalic T, Whiteley E, Vowles J, Giustacchini A, Argoud K, Sopp P, Nakanishi M, Bowden R, Cowley S, Newey S, Akerman C, Ponting CP, Cader MZ. Assessing similarity to primary tissue and cortical layer identity in induced pluripotent stem cell-derived cortical neurons through single-cell transcriptomics. Hum Mol Genet. 2016;25(5):989-1000.

${ }^{216}$ Sakaguchi H, Kadoshima T, Soen M, Narii N, Ishida Y, Ohgushi M, Takahashi J, Eiraku M, Sasai Y. Generation of functional hippocampal neurons from self-organizing human embryonic stem cell-derived dorsomedial telencephalic tissue. Nat Commun. 2015;6:8896.

${ }^{217}$ Liu H, Zhang SC. Specification of neuronal and glial subtypes from human pluripotent stem cells. Cell Mol Life Sci. 2011;68(24):3995-4008.

218 Steffek A.E., McCullumsmith R.E., Haroutunian V., Meador-Woodruff J.H. Cortical expression of glial fibrillary acidic protein and glutamine synthetase is decreased in schizophrenia. Schizophr. Res. 2008;103:71-82.

${ }^{219}$ Williams MR, Hampton T, Pearce RK, Hirsch SR, Ansorge O, Thom M, Maier M. Astrocyte decrease in the subgenual cingulate and callosal genu in schizophrenia. Eur Arch Psychiatry Clin Neurosci. 2013;263(1):41-52.

220 Webster MJ, Knable MB, Johnston-Wilson N, Nagata K, Inagaki M, Yolken RH.Immunohistochemical localization of phosphorylated glial fibrillary acidic protein in the prefrontal cortex and hippocampus from patients with schizophrenia, bipolar disorder, and depression. Brain Behav Immun. 2001;15(4):388-400.

${ }^{221}$ Fatemi SH, Laurence JA, Araghi-Niknam M, Stary JM, Schulz SC, Lee S, Gottesman II. Glial fibrillary acidic protein is reduced in cerebellum of subjects with major depression, but not schizophrenia. Schizophr Res. 2004;69(2-3):317-23.

${ }^{222}$ Toro CT, Hallak JE, Dunham JS, Deakin JF. Glial fibrillary acidic protein and glutamine synthetase in subregions of prefrontal cortex in schizophrenia and mood disorder. Neurosci Lett. 2006;404(3):276-81.

${ }^{223}$ Radomska KJ, Halvardson J, Reinius B, Lindholm Carlström E, Emilsson L, Feuk L, Jazin E. RNA-binding protein QKI regulates Glial fibrillary acidic protein expression in human astrocytes. Hum Mol Genet. 2013;22(7):1373-82.

${ }^{224}$ Guo QH, Yang HJ, Wang SD. Olanzapine inhibits the proliferation and induces the differentiation of glioma stem-like cells through modulating the Wnt signaling pathway in vitro. Eur Rev Med Pharmacol Sci. 2015;19(13):2406-15.

${ }^{225}$ Castellano O, Moscoso A, Riolobos AS, Carro J, Arji M, Molina V, López DE, Sancho C. Chronic administration of risperidone to healthy rats: a behavioural and morphological study. Behav Brain Res. 2009;205(2):488-98.

${ }^{226}$ Fatemi SH, Folsom TD, Reutiman TJ, Pandian T, Braun NN, Haug K. Chronic psychotropic drug treatment causes differential expression of connexin 43 and GFAP in frontal cortex of rats. Schizophr Res. 2008;104(1-3):127-34.

227 Allaman I, Fiumelli H, Magistretti PJ, Martin JL. Fluoxetine regulates the expression of neurotrophic/growth factors and glucose metabolism in astrocytes. Psychopharmacology (Berl). 2011;216(1):75-84.

${ }^{228}$ Kettenmann, Ramson. Neuroglia. 2005. Oxforg University Press. 
${ }^{229}$ Quesseveur G, Gardier AM, Guiard BP. The monoaminergic tripartite synapse: a putative target for currently available antidepressant drugs. Curr Drug Targets. 2013;14(11):1277-94.

${ }^{230}$ Pin JP, Duvoisin R. The metabotropic glutamate receptors: structure and functions. Neuropharmacology. 1995;34(1):1-26.

${ }^{231}$ Shigemoto R, Kinoshita A, Wada E, Nomura S, Ohishi H, Takada M, Flor PJ, Neki A, Abe T, Nakanishi S, Mizuno N. Differential presynaptic localization of metabotropic glutamate receptor subtypes in the rat hippocampus. J Neurosci. 1997;17(19):7503-22.

${ }^{232}$ Takahashi T, Forsythe ID, Tsujimoto T, Barnes-Davies M, Onodera K. Presynaptic calcium current modulation by a metabotropic glutamate receptor. Science. 1996;274(5287):594-7.

${ }^{233}$ Fell MJ, Svensson KA, Johnson BG, Schoepp DD. Evidence for the role of metabotropic glutamate (mGlu) 2 not mGlu3 receptors in the preclinical antipsychotic pharmacology of the mGlu2/3 receptor agonist (-)-(1R,4S,5S,6S)-4-amino-2-sulfonylbicyclo[3.1.0] hexane-4,6dicarboxylic acid (LY404039). J Pharmacol Exp Ther. 2008;326(1):209-17.

${ }^{234}$ Kent JM, Daly E, Kezic I, Lane R, Lim P, De Smedt H, De Boer P, Van Nueten L, Drevets WC, Ceusters M. Efficacy and safety of an adjunctive mGlu2 receptor positive allosteric modulator to a SSRI/SNRI in anxious depression. Prog Neuropsychopharmacol Biol Psychiatry. 2016;67:66-73.

${ }^{235}$ Meinhardt MW, Hansson AC, Perreau-Lenz S, Bauder-Wenz C, Stählin O, Heilig M, Harper C, Drescher KU, Spanagel R, Sommer WH. Rescue of infralimbic mGluR2 deficit restores control over drug-seeking behavior in alcohol dependence. $J$ Neurosci. 2013;33(7):2794-806.

${ }^{236}$ González-Maeso J, Ang RL, Yuen T, Chan P, Weisstaub NV, López-Giménez JF, Zhou M, Okawa Y, Callado LF, Milligan G, Gingrich JA, Filizola M, Meana JJ, Sealfon SC. Identification of a serotonin/glutamate receptor complex implicated in psychosis. Nature. 2008;452(7183):93-7.

${ }^{237}$ Kurita M, Holloway T, García-Bea A, Kozlenkov A, Friedman AK, Moreno JL, Heshmati M, Golden SA, Kennedy PJ, Takahashi N, Dietz DM, Mocci G, Gabilondo AM, Hanks J, Umali A, Callado LF, Gallitano AL, Neve RL, Shen L, Buxbaum JD, Han MH, Nestler EJ, Meana JJ, Russo SJ, González-Maeso J. HDAC2 regulates atypical antipsychotic responses through the modulation of mGlu2 promoter activity. Nat Neurosci. 2012;15(9):1245-54.

${ }^{238}$ Miyamoto S, Duncan GE, Marx CE, Lieberman JA. Treatments for schizophrenia: a critical review of pharmacology and mechanisms of action of antipsychotic drugs. Mol Psychiatry. 2005;10(1):79-104.

${ }^{239}$ Li QH, Nakadate K, Tanaka-Nakadate S, Nakatsuka D, Cui Y, Watanabe Y. Unique expression patterns of 5-HT2A and 5-HT2C receptors in the rat brain during postnatal development: Western blot and immunohistochemical analyses. J Comp Neurol. 2004;469(1):128-40.

${ }^{240}$ Patil ST, Zhang L, Martenyi F, Lowe SL, Jackson KA, Andreev BV, Avedisova AS, Bardenstein LM, Gurovich IY, Morozova MA, Mosolov SN, Neznanov NG, Reznik AM, Smulevich AB, Tochilov VA, Johnson BG, Monn JA, Schoepp DD. Activation of mGlu2/3 receptors as a new approach to treat schizophrenia: a randomized Phase 2 clinical trial. Nat Med. 2007;13(9):1102-7.

${ }^{241}$ Wilson GM, Flibotte S, Chopra V, Melnyk BL, Honer WG, Holt RA. DNA copy-number analysis in bipolar disorder and schizophrenia reveals aberrations in genes involved in glutamate signaling. Hum Mol Genet. 2006;15(5):743-9

${ }^{242}$ Wellcome Trust Case Control Consortium. Genome-wide association study of 14,000 cases of seven common diseases and 3,000 shared controls. Nature. 2007;447(7145):661-78. 
${ }^{243}$ Ohtsuki T, Koga M, Ishiguro H, Horiuchi Y, Arai M, Niizato K, Itokawa M, Inada T, Iwata N, Iritani S, Ozaki N, Kunugi H, Ujike H, Watanabe Y, Someya T, Arinami T. A polymorphism of the metabotropic glutamate receptor mGluR7 (GRM7) gene is associated with schizophrenia. Schizophr Res. 2008;101(1-3):9-16.

${ }^{244}$ Losonczy A, Somogyi P, Nusser Z. Reduction of excitatory postsynaptic responses by persistently active metabotropic glutamate receptors in the hippocampus. J Neurophysiol. 2003;89(4):1910-9.

${ }^{245}$ Klar R, Walker AG, Ghose D, Grueter BA, Engers DW, Hopkins CR, Lindsley CW, Xiang Z, Conn PJ, Niswender CM. Activation of Metabotropic Glutamate Receptor 7 Is Required for Induction of Long-Term Potentiation at SC-CA1 Synapses in the Hippocampus. J Neurosci. 2015;35(19):7600-15.

${ }^{246}$ Giustizieri M, Bernardi G, Mercuri NB, Berretta N. Distinct mechanisms of presynaptic inhibition at GABAergic synapses of the rat substantia nigra pars compacta. J Neurophysiol. 2005;94(3):1992-2003.

${ }^{247}$ Domin H, Jantas D, Śmiałowska M. Neuroprotective effects of the allosteric agonist of metabotropic glutamate receptor 7 AMN082 on oxygen-glucose deprivation- and kainateinduced neuronal cell death. Neurochem Int. 2015;88:110-23.

${ }^{248}$ Greco B, Lopez S, van der Putten H, Flor PJ, Amalric M. Metabotropic glutamate 7 receptor subtype modulates motor symptoms in rodent models of Parkinson's disease. J Pharmacol Exp Ther. 2010;332(3):1064-71.

${ }^{249}$ Konieczny J, Lenda T. Contribution of the mGluR7 receptor to antiparkinsonian-like effects in rats: a behavioral study with the selective agonist AMN082. Pharmacol Rep. 2013;65(5):1194-203.

${ }^{250} \mathrm{Li}$ X, Markou A. Metabotropic Glutamate Receptor 7 (mGluR7) as a Target for the Treatment of Psychostimulant Dependence. CNS Neurol Disord Drug Targets. 2015;14(6):738-44.

${ }^{251}$ Wojcik SM, Rhee JS, Herzog E, Sigler A, Jahn R, Takamori S, Brose N, Rosenmund C. An essential role for vesicular glutamate transporter 1 (VGLUT1) in postnatal development and control of quantal size. Proc Natl Acad Sci U S A. 2004;101(18):7158-63.

${ }^{252}$ Machado NJ, Simões AP, Silva HB, Ardais AP, Kaster MP, Garção P, Rodrigues DI, Pochmann D, Santos AI, Araújo IM, Porciúncula LO, Tomé ÂR, Köfalvi A, Vaugeois JM, Agostinho P, El Yacoubi M, Cunha RA, Gomes CA. Caffeine Reverts Memory But Not Mood Impairment in a Depression-Prone Mouse Strain with Up-Regulated Adenosine A(2A) Receptor in Hippocampal Glutamate Synapses. Mol Neurobiol. 2016 Feb 9.

${ }^{253}$ Pan PY, Marrs J, Ryan TA. Vesicular glutamate transporter 1 orchestrates recruitment of other synaptic vesicle cargo proteins during synaptic vesicle recycling. J Biol Chem. 2015;290(37):22593-601.

${ }^{254}$ Cheng XR, Yang Y, Zhou WX, Zhang YX. Expression of VGLUTs contributes to degeneration and acquisition of learning and memory. Neurobiol Learn Mem. 2011;95(3):361-75.

${ }^{255}$ Ni B, Du Y, Wu X, DeHoff BS, Rosteck PR Jr, Paul SM. Molecular cloning, expression, and chromosomal localization of a human brain-specific $\mathrm{Na}(+)$-dependent inorganic phosphate cotransporter. J Neurochem. 1996;66(6):2227-38.

${ }^{256}$ Uezato A, Meador-Woodruff JH, McCullumsmith RE. Vesicular glutamate transporter mRNA expression in the medial temporal lobe in major depressive disorder, bipolar disorder, and schizophrenia. Bipolar Disord. 2009;11(7):711-25.

${ }^{257}$ Oni-Orisan A, Kristiansen LV, Haroutunian V, Meador-Woodruff JH, McCullumsmith RE. Altered vesicular glutamate transporter expression in the anterior cingulate cortex in schizophrenia. Biol Psychiatry. 2008;63(8):766-75. 
${ }^{258}$ Varea E, Guirado R, Gilabert-Juan J, Martí U, Castillo-Gomez E, Blasco-Ibáñez JM, Crespo C, Nacher J. Expression of PSA-NCAM and synaptic proteins in the amygdala of psychiatric disorder patients. J Psychiatr Res. 2012;46(2):189-97.

${ }^{259}$ Eastwood SL, Harrison PJ. Decreased expression of vesicular glutamate transporter 1 and complexin II mRNAs in schizophrenia: further evidence for a synaptic pathology affecting glutamate neurons. Schizophr Res. 2005;73(2-3):159-72.

${ }^{260}$ Moutsimilli L, Farley S, Dumas S, El Mestikawy S, Giros B, Tzavara ET. Selective cortical VGLUT1 increase as a marker for antidepressant activity. Neuropharmacology. 2005;49(6):890-900.

${ }^{261}$ Santostefano KE, Hamazaki T, Biel NM, Jin S, Umezawa A, Terada N. A practical guide to induced pluripotent stem cell research using patient samples. Lab Invest. 2015;95(1):4-13.

${ }^{262}$ Martins-Taylor K, Nisler BS, Taapken SM, Compton T, Crandall L, Montgomery KD, Lalande M, Xu RH. Recurrent copy number variations in human induced pluripotent stem cells. Nat Biotechnol. 2011;29(6):488-91.

${ }^{263}$ Dolmetsch R, Geschwind DH. The human brain in a dish: the promise of iPSC-derived neurons. Cell. 2011;145(6):831-4.

${ }^{264}$ Illes S, Theiss S, Hartung HP, Siebler M, Dihné M. Niche-dependent development of functional neuronal networks from embryonic stem cell-derived neural populations. BMC Neurosci. 2009;10:93.

${ }^{265}$ Ge S, Sailor KA, Ming GL, Song H. Synaptic integration and plasticity of new neurons in the adult hippocampus. J Physiol. 2008;586(16):3759-65

266 Johnson MA, Weick JP, Pearce RA, Zhang SC. Functional neural development from human embryonic stem cells: accelerated synaptic activity via astrocyte coculture. J Neurosci. 2007;27(12):3069-77.

${ }^{267}$ Tiemann U, Wu G, Marthaler AG, Schöler HR, Tapia N. Epigenetic Aberrations Are Not Specific to Transcription Factor-Mediated Reprogramming. Stem Cell Reports. 2016;6(1):3543.

${ }^{268}$ Eastwood SL, Burnet PW, Harrison PJ. Expression of complexin I and II mRNAs and their regulation by antipsychotic drugs in the rat forebrain. Synapse. 2000;36(3):167-77.

${ }^{269}$ Kapur S, VanderSpek SC, Brownlee BA, Nobrega JN. Antipsychotic dosing in preclinical models is often unrepresentative of the clinical condition: a suggested solution based on in vivo occupancy. J Pharmacol Exp Ther. 2003;305(2):625-31.

${ }^{270}$ Groshong R, Baldessarini RJ, Gibson DA, Lipinski JF, Axelrod D, Pope A.

Activities of types A and B MAO and catechol-o-methyltransferase in blood cells and skin fibroblasts of normal and chronic schizophrenic subjects. Arch Gen Psychiatry. 1978;35(10):1198-1205.

${ }^{271}$ Gassó P, Mas S, Molina O, Lafuente A, Bernardo M, Parellada E. Increased susceptibility to apoptosis in cultured fibroblasts from antipsychotic-naïve first-episode schizophrenia patients. J Psychiatr Res. 2014;48(1):94-101.

${ }^{272}$ Catts VS, Catts SV, McGrath JJ, Féron F, McLean D, Coulson EJ, Lutze-Mann LH. Apoptosis and schizophrenia: a pilot study based on dermal fibroblast cell lines. Schizophr Res. 2006;84(1):20-8. 


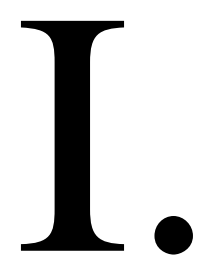




\title{
Metabolic stress-induced microRNA and mRNA expression profiles of human fibroblasts
}

\author{
Sára Kálmán ${ }^{1}$, Krassimira Garbett ${ }^{2}$, Andrea Vereczkei ${ }^{1,3}$, Richard C. Shelton ${ }^{4}$, Zeljka \\ Korade $^{1,5}$, and Károly Mirnics ${ }^{1,2,5,{ }^{*}}$ \\ ${ }^{1}$ Department of Psychiatry, University of Szeged, Szeged, Hungary \\ 2Department of Psychiatry, Vanderbilt University, Nashville, TN, USA \\ ${ }^{3}$ Institute for Medical Chemistry, Molecular Biology and Pathobiochemistry, Semmelweis \\ University, Budapest, Hungary \\ ${ }^{4}$ Department of Psychiatry, University of Alabama, Birmingham, AL, USA \\ ${ }^{5}$ Vanderbilt Kennedy Center for Research on Human Development, Vanderbilt University, \\ Nashville, TN, USA
}

\section{Abstract}

Metabolic and oxidative stresses induce physiological adaptation processes, disrupting a finely tuned, coordinated network of gene expression. To better understand the interplay between the mRNA and miRNA transcriptomes, we examined how two distinct metabolic stressors alter the expression profile of human dermal fibroblasts.

Primary fibroblast cultures were obtained from skin biopsies of 17 healthy subjects. Metabolic stress was evoked by growing subcultured cells in glucose deprived, galactose enriched (GAL) or lipid reduced, cholesterol deficient (RL) media, and compared to parallel-cultured fibroblasts grown in standard (STD) medium. This was followed by mRNA expression profiling and assessment of $>1,000$ miRNAs levels across all three conditions. The miRNA expression levels were subsequently correlated to the mRNA expression profile.

Metabolic stress by RL and GAL both produced significant, strongly correlated mRNA/miRNA changes. At the single gene level four miRNAs (miR-129-3p, miR-146b-5p, miR-543 and miR-550a) showed significant and comparable expression changes in both experimental conditions. These miRNAs appeared to have a significant physiological effect on the transcriptome, as nearly $10 \%$ of the predicted targets reported changes at mRNA level. The two distinct metabolic stressors induced comparable changes in the miRNome profile, suggesting a common defensive response of the fibroblasts to altered homeostasis. The differentially expressed miR-129-3p, miR-146b-5p, miR-543 and miR-550a regulated multiple genes (e.g. NGEF, NOVA1, PDE5A) with region- and age-specific transcription in the human brain, suggesting that deregulation of these miRNAs might have significant consequences on CNS function. The overall findings suggest that analysis of stress-induced responses of peripheral fibroblasts, obtained from patients with psychiatric disorders is a promising avenue for future research endeavors.

(C) 2013 Elsevier Inc. All rights reserved

"Correspondence: Karoly Mirnics, Department of Psychiatry, Vanderbilt University, 8130A MRB III, 465 21st Avenue South, Nashville TN 37232, USA, karoly.mirnics@ vanderbilt.edu, Office phone: 615-936-1074, http://mirnicslab.vanderbilt.edu/mirnicslab/.

Publisher's Disclaimer: This is a PDF file of an unedited manuscript that has been accepted for publication. As a service to our customers we are providing this early version of the manuscript. The manuscript will undergo copyediting, typesetting, and review of the resulting proof before it is published in its final citable form. Please note that during the production process errors may be discovered which could affect the content, and all legal disclaimers that apply to the journal pertain. 


\section{Keywords}

human fibroblast; stress; miRNA; mRNA; profiling; lipid; galactose; qPCR

\section{INTRODUCTION}

Postmortem brain studies of psychiatric disorders show complex transcriptome profile changes that encompass both miRNA and mRNA disturbances [1,2]. Yet, these studies face considerable confounds, limiting mechanistic and molecular investigation into the causality of the observed changes. The uniquely human occurrence of major psychiatric disorders and the absence of bona fide animal models $[3,4,5]$ underscore the need for analyzing human tissue. However, the usefulness of post-mortem brain tissue is limited primarily to static measures, such as gene expression analysis. Patient fibroblasts, due to their abundance, availability, and neuron-like receptor and protein expression are appealing alternative in vitro models for functional analysis of brain disorders $[6,7]$.

Stress is a strong modulator of gene expression [8]. In vitro, glucose-depleted, galactosesupplemented medium is a widely used metabolic stress treatment in fibroblast cultures [9]: glucose depletion increases the level of reactive-oxygen species (ROS), which results in elevated, compensatory glutathione production [7]. Yet, over a prolonged time starvation results in reduction of NADPH, limiting the efficacy of the glutathione protection to the cells. In contrast, reduction in the exogenous cholesterol is a different kind, but related metabolic stress: to maintain their membrane structure and survive, fibroblasts are forced to synthesize their own cholesterol, stressing their metabolic machinery [10]. These two metabolic stress models are likely to share common characteristics: there is an increased rate of glucose incorporation into lipid when cells are switched into lipid-deficient medium [11] and $\mathrm{C}^{14}$ galactose incorporates into galactolipids [12]. Therefore, both would be expected to manifest common disturbances in the gene expression machinery.

Micro-RNAs (miRNA) are 18-22 nucleotide long fine-tuning regulators of gene expression and protein production [13]. Thousands of miRNAs are encoded in the human genome, controlling the function of approximately $50 \%$ of our genes. miRNA expression is tissue, environment and individual specific [14]. A single miRNA can regulate the expression of hundreds of mRNAs, cause their degradation, destabilization, stocking or transport.

The present study was aimed to answer three questions. First, do the different metabolic stressors alter the miRNA expression profile of human fibroblasts? Second, are the miRNA expression profiles similar between two different metabolic stressors? Finally, do the miRNA expression changes result in changes at the mRNA level? Importantly, these studies provide critical baseline data for similar experiments that will be performed on fibroblasts from patients with schizophrenia, major depression and bipolar disorder.

\section{MATERIALS AND METHODS ETHICS STATEMENT}

The Vanderbilt University Institutional Review Board (IRB) approved the study and written informed consent was obtained from all study participants before any procedures were conducted.

\section{HUMAN FIBROBLAST CELL CULTURES}

Specimens for fibroblast cultures were obtained by skin biopsies $(\sim 1 \times 2 \mathrm{~mm})$ from 17 healthy subjects (4 males and 13 females) (Supplemental Material 1) as described previously 
[15]. Exclusion criteria included any DSM-IV diagnosis [16] or any medical condition that would preclude the biopsy.

All fibroblast samples used in this experiment were cultured between passages 5 and 10 using Dulbecco's modified Eagle's medium ${ }^{\mathrm{TM}}$ (DMEM; Mediatech, Manassas, VA, USA) containing $25 \mathrm{mM}$ glucose, $1 \mathrm{mM}$ sodium pyruvate supplemented with $2 \mathrm{mM}$ L-glutamine (Mediatech), 10\% fetal bovine serum (FBS; Thermo Scientific HyClone, Logan, UT, USA) and antibiotic/antimycotic solution (A/A; Invitrogen) at $37{ }^{\circ} \mathrm{C}$ and $5 \% \mathrm{CO}_{2}$. The cells were cultured simultaneously under same conditions until reaching confluence, trypsinized and counted.

A single cell line from each individual was divided into three plates (plating density $1.2 \times 106$ cells/plate) and after overnight adherence, the medium was changed into 3 different conditions. All cultures were grown for 7 days, with a media change every 2 days. (Figure 1) and were grown in: 1) lipid reduced (RL) medium (DMEM supplemented with lipid reduced FBS (Thermo Scientific HyClone, Logan, UT, USA)); 2) galactose-containing (GAL) medium (DMEM deprived of glucose (Mediatech) supplemented with $10 \mathrm{mM}$ galactose) and 3) standard, glucose-containing (STD) medium. The medium was changed three times a week. Cell growth and proliferation were not affected by GAL or RL treatment. After one week the cells were washed with ice-cold PBS twice, trypsinized, pelleted (700g for $8 \mathrm{~min}$ on $18{ }^{\circ} \mathrm{C}$ ) and stored on $-80^{\circ} \mathrm{C}$ until the RNA isolation.

\section{RNA ISOLATION}

Total cell RNA and small RNA fractions were isolated from the frozen samples using the mirVana miRNA isolation Kit (Ambion, Austin, TX) according to manufacturer's instructions. In brief, the pellet was suspended in $600 \mu \mathrm{l}$ Lysis/Binding Solution, than $60 \mu \mathrm{l}$ Homogenate Additive was added. After 10 min incubation time for total RNA isolation $1.25 \times$ volume of $100 \%$ ethanol was added to the aqueous phase and the supernatant was purified using the proprietary solutions provided by the manufacturer. Finally the total RNA was collected with $100 \mu$ l Elution Solution. For small RNA isolation we added on-thrid volume of $100 \%$ ethanol to the aqueous phase and two-thrid volume of $100 \%$ ethanol to the flow-through. The small RNA specied were collected from the filter with $100 \mu$ l Elution Solution. Agilent 2100 Bioanalyzer was used to determine the quality and size distribution of the total RNA and small RNA fractions. All samples showed a RNA Intergity Number (RIN) >8.0. The samples were stored on $-80^{\circ} \mathrm{C}$ until used.

\section{DNA MICROARRAY and miRNome PCR ARRAY}

mRNA expression-mRNA levels were examined with the GeneChip HT HG-U133+ PM Array Plate (Affymetrix, Santa Clara, CA, USA). cDNA synthesis from total RNA, IVT, labeling and hybridization were performed according to manufacturer's protocol.

miRNA expression-From small RNA samples cDNA was prepared with miScript II RT Kit (Qiagen, Valencia, CA, USA) using miScript HiSpec Buffer. The DNA was quantified with NanoDrop ND-1000 spectophotometer, yielding 693-839 ng/ $\mu \mathrm{l}$. After reverse transcription, the individual sample cDNAs were pooled into four groups based the gender and the age. Each group contained material from 4-5 individuals. Group 1: males age of 30-48; group 2: females age of 20-25; group 3: females age of 27-40; group 4: females age of 44-52 (Supplemental Material 1). The relative amounts of 1008 miRNAs were measured with miRNome miRNA PCR Arrays using miScript SYBR Green PCR Kit (Qiagen, Valencia, CA, USA) according to the manufacturer's instructions. 


\section{VALIDATION OF mRNA FINDINGS}

Based on our pathway analyses we selected a panel of 10 lipid biosynthesis genes, and validated the mRNAs using qPCR on the individual control, GAL-treated and RL-exposed samples $(\mathrm{n}=18)$. Total RNA (500 ng) from each sample was reverse transcribed to cDNA using RT2 First Strand Kit (Qiagen) using the instructions of the manufacturer. Customdesigned RT2 Profiler PCR Arrays (Qiagen) were provided in 96-well plate format and contained primer assays for 10 focused genes (SCD, FABP3, DHCR7, INSIG1, QPRT, HMGCR, HMGCS1, LDLR, FADS1, FADS2) and 2 housekeeping genes (ACTB and GAPDH). RT2 Profiler PCR Arrays assays were performed on the real-time cycler ABI Prism 7300 System (Applied Biosystems). The exported Cts were analyzed using SABiosciences PCR Array Data Analysis Template Excel software and compared across STD-RL and STD-GAL groups using a paired experimental design.

\section{VALIDATION OF mIRNA FINDINGS}

22 miRNAs were selected for follow up on the 17 individual samples (hsa-miR-146b-5p, hsa-miR-550a, hsa-miR-129-3p, hsa-miR-214, hsa-miR-21, hsa-miR-22, hsa-miR-132, hsamiR-376c, hsa-miR-19a, hsa-miR-195, hsa-miR-181a, hsa-miR-486-5p, hsa-miR-377, hsamiR-424,hsa-miR-542-3p,hsa-miR-22, hsa-miR-103a, hsa-miR-376b, hsa-miR-29b, hsamiR-185, hsa-miR-564, hsa-miR-34a). The assays was identical to the one used on the pooled samples described above.

\section{DATA ANALYSIS}

Differential expression-For the mRNA studies, after standard image segmentation of the microarray images, RMA normalization was performed using GenePattern software [17]. At the individual level, the gene was considered to be differentially expressed if it showed an absolute average log ratio $|A L R| \geq 0.378$ and $p$-value $\leq 0.01$ between the experimental and control conditions. Pathway analyses were performed using GenePattern Gene Set Enrichment Analysis (GSEA) [18] and its BioCarta Database.

For miRNA expression quantification comparative Ct method was used with SNORD61, SNORD68, SNORD72, SNORD95, SNORD96A and RNU6-2 as housekeeping genes. A miRNA was considered differentially expressed if it showed a $|\Delta \Delta \mathrm{Ct}| \geq 0.583$ and both a groupwise and pairwise $p$ value of $p \leq 0.05$ between the experimental and control condition.

These parameters were chosen based on previously analyzed datasets $[17,18]$, and followed up by qPCR validation.

Hierarchical clustering and calculation of correlation-For both the mRNA and miRNA data two-way unsupervised clustering using Euclidian distance was performed using the GenePattern software package [19]. For mRNA, this was performed using the RMA - normalized $\log _{2}$ gene expression values from each individual sample, while for the miRNA the $\Delta \mathrm{Ct}$ values were used. Correlations were calculated in MS-Excel 2010 using Pearson coefficient.

Identification of miRNA targets-To identify the potentially affected mRNAs by the four miRNAs that were changed in both the GAL- and RL-exposed cultures (miR-129-3p, miR-146b-5p, miR-543 and miR-550a), we searched the miRDB online database [21] and established the list of their potential targets. 


\section{COMPARISON TO BRAIN EXPRESSION}

To test the relevance of our fibroblast findings in context of human brain disorders, we examined the mRNA expression of putative miR-129-3p, miR-146b-5p, miR-543 and miR-550a targets in the developing human brain [33].

\section{RESULTS}

\section{METABOLIC STRESS-INDUCED mRNA CHANGES}

Our mRNA expression profiling revealed 2063 differentially expressed genes in the GAL group compared to the matched controls. Of these, 1117 (54.1\%) were up-regulated (Supplemental Material 2A), while 946 (45.9\%) showed downregulation. These regulated gene products were involved in cell cycle, apoptosis, inflammatory response and mRNA processing related pathways, and in metabolic adaptation. RL treatment had also a widespread effect on the transcriptome. 984 gene transcripts were affected by the RL treatment, with 640 mRNAs increased (65.0\%) and 344 (35.0\%) transcripts repressed (Supplemental Material 2B). Two-way unsupervised hierarchical clustering provided a good separation of samples based on the treatment condition (Supplemental Material 3AB). Pathway analysis with BioCarta classification revealed enrichment of 18 groups of transcripts in the GAL condition (Table 1A), while in the RL condition, the analysis revealed enrichment in 12 pathways and transcript repression in 3 pathways (Table 1B). Notably, 4 of these pathways were commonly upregulated in both the GAL and RL condition: peroxisome proliferator-activated receptor a (PPARA), carbohydrate response element binding protein 2 (CHREBP2), influence of Ras and Rho proteins on G1 to S Transition (RACCYCD) and stress induction of HSP regulation (HSP27). As there was a significant enrichment of lipid biosynthesis genes in the fibroblast as a result of exposure to RL and GAL conditions, we decided to validate a panel 10 commonly upregulated genes (SCD, FABP3, DHCR7, INSIG1, QPRT, HMGCR, HMGCS1, LDLR, FADS1, FADS2). qPCR assessment of expression validated 9 out of 10 transcript changes in both RL and GAL conditions (all except HMGCR, which showed the same directionality and magnitude of change, but did not reach statistical significance) (Supplemental Material 4) and revealed a high correlation between the microarray and qPCR results (GAL-STD comparison: $r=0.92$; RL-STD comparison: $r=0.72$ ) (Figure 2).

\section{miRNA EXPRESSION IN RESPONSE TO METABOLIC STRESS}

We found that in human fibroblasts 45 miRNAs were differentially expressed due to the GAL treatment (Table $2 \mathrm{~A}, \mathrm{~B})(|\Delta \Delta \mathrm{Ct}| \geq 0.583 ; p \unlhd 0.05)$, with approximately same number of miRNAs increased and decreased ( 24 miRNAs and 21 miRNAs, respectively). The RL treatment affected 34 miRNAs, with more prominent downregulation than upregulation (27 and 7, respectively). When compared to the control cultures, four miRNAs showing significant differential expression overlapped in the two metabolic stress conditions (GAL, RL) (Figure 3). However, the overall pattern of miRNA profiles between the two stressors was strong: the differentially expressed miRNA's in the GAL condition, when correlated with their expression level in the RL condition, showed a significant correlation ( $\mathrm{r}=0.71$, $\mathrm{p}<0.001$ ) (Figure 4A). Similarly, the expression levels of differentially expressed miRNA's in the RL condition were correlated with the expression levels observed in the RL condition $(r=0.65, p<0.005)$ (Figure 4B). Furthermore, the combined list of these miRNA expression changes, when subjected to a two-way unsupervised hierarchical clustering, perfectly separated out the CNT, GAL and RL conditions (Figure 5). Thus, while the most changed miRNAs were not identical between the GAL and RL, the overall pattern suggested a comparable miRNA response between the two very different metabolic stressors. 
As the initial screening was performed on 4 pools of samples which could theoretically bias our data, we wanted to validate the expression of 22 miRNAs on our 17 individual samples. In this follow-up study the initial miRNA findings on the pooled samples showed the expected expression pattern on the individual experimental samples (Figure 6).

\section{CORRELATION OF MIRNA WITH MRNA EXPRESSION}

To establish how metabolic stress-induced miRNAs affect the mRNA transcriptome, we focused our attention on the four significantly changed miRNAs that overlapped between the GAL- and RL-treatment: miR-129-3p, miR-146b-5p, miR-543 and miR-550a. To identify the target mRNAs of these miRNAs we established the list of their potential targets using the miRDB online data base. The database revealed 211 putative mRNA targets of miR-129-3p, 243 mRNA targets of miR146b-5p, 129 mRNA targets of miR-550a, and 921 targets for miR-543 (Supplemental Material 5). Of the combined 1504 potential targets 140 mRNAs showed significant change at gene expression level (Table 3A-C). It is noteworthy that multiple target genes showed significantly altered transcription levels in both the GALand RL-treated human fibroblast cultures, further arguing for a consistent effect of the two different metabolic stressors.

Finally, we attempted to establish if any of these putative mRNA targets of miR-129-3p, miR-146b-5p, miR-543 and miR-550a showed spatio-temporal regulation in the developing human brain [31]. Fourteen such transcripts were identified (rfx7, syt14, nova1, kiaa1199, spata13, osbp18, exph5, ngef, pag1, acyp1, pde5a, adamts11, cyp26b1 and pag1) (Supplemental Material 6), suggesting that these targets should be the primary focus of further follow-up examinations in the brain tissue of neurodevelopmental disorders.

\section{CONCLUSIONS}

This study established the mRNA and miRNA expression changes in human fibroblast cell cultures in response to two nutrition-based metabolic stress paradigms. We found that 1) glucose depleted, galactose supplemented and a lipid reduced, cholesterol depleted medium produced widespread mRNA and miRNA changes in human fibroblasts; 2) while the most significantly changed miRNAs showed variation, the overall miRNA changes across the two metabolic stress conditions showed considerable similarity; 3 ) it appears that changes in miRNA expression contributed considerably to altered mRNA expression profile in both the GAL and RL conditions and 4) we identified 4 significantly changed miRNAs (miR-129-3p, miR-146b-5p, miR-543 and miR-550a) that are likely to be important mediators of multiple types of metabolic stress.

Using the individual transcript assessment approach, we identified only four significantly changed miRNAs (miR-129-3p, miR-146b-5p, miR-543 and miR-550a) across the two metabolic stressors. Yet, this is not surprising, as transcripts work in networks: while we observed considerable differences in the most changed miRNAs of the GAL- and RL-treated fibroblasts, the overall miRNA profile of the two stressors was strikingly similar. Furthermore, 4 metabolic stress-related transcript pathways (PPARA, CHREBP2, RACCYCD, and HSP27) were upregulated in both GAL and RL-exposed cultures, suggesting that this common signature is present both at the miRNA and mRNA level. This came as a surprise, as the two stressors acts through distinct mechanisms: the GAL condition primarily creates oxidative radicals through starvation [7], while the RL conditions forces the cells into a metabolic overdrive [10]. Based on these findings we propose that metabolic stress, regardless of the type of the insult, might have a common miRNome and mRNA signature, and this common signature perhaps represents a non-specific resilience response, helping the cells to adapt and survive. 
Our results also suggest that the four changed miRNAs alone appeared to be able to regulate the mRNA expression of 52 target genes, arguing that miRNA regulation of the transcriptome might be a significant contributor to metabolic stress-induced cellular responses. Unfortunately, miR-543 and miR-550a has not been well studied experimentally, and their effects remain mostly unknown. However, mir-129-2 and miR-146b-5p have been a focus of multiple investigations to date: mir-129-2 is located next to a $\mathrm{CpG}$ island and is methylation-associated, and the expression of this miRNA can be epigenetically restored by de-methylating drugs [22]. Furthermore, mir-129-2 is conserved in vertebrates and regulates the expression of CP110, ARP2, TOCA1, ABLIM1 and ABLIM3, thus effectively controlling actin dynamics in the cell [21]. Still, miR-146b-5p might be the most important finding of our studies: its expression is highly induced by proinflammatory cytokines, with a clear dependency on IFN- $\gamma$, potentially via the JAK/STAT pathway $[24,25]$. Furthermore, a decrease of miR-146b-5p has been observed in monocytes during obesity, and it was associated with loss of the anti-inflammatory signaling action [26]. miR-146b-5p also regulates signal transduction of TGF- $\beta$ [27], and appears to play a critical role in the pathophysiology of various cancers $[28,29]$. These data suggest that metabolic stress might regulate miRNA expression through epigenetic modification, and that the two metabolic stressors trigger an inflammation-like response in the stressed cells. However, this hypothesis has to be tested more comprehensively in follow-up experiments.

Finally, we need to emphasize that peripheral biomarkers studies are essential to understand the pathophysiology of human brain disorders. Recent studies indicate that peripheral biomarkers may aid the diagnosis of psychiatric disorders and help assess treatment efficacy [30]. However, obtaining peripheral patient tissues and analyzing them at resting state might not be sufficient to reveal the critical deficits, and they must be challenged to reveal the full signature of the disease [7,31]. Human dermal fibroblasts as a model system are ideally suited for such studies [32], as they have several advantages over the functional assays performed on peripheral blood cells: 1) they can be readily propagated without immortalization, 2) have a more similar expression profile to brain cells than peripheral blood cells; 3 ) are homogenous and very responsive to a variety of stimuli, and 4) they are minimally affected by epigenetic changes, which usually disappear after the $5^{\text {th }}$ culture passage.

Our results also suggest that metabolic stress in dermal fibroblasts affects genes that are important for brain development and homeostasis. The 4 miRNAs with differential expression in both the RL and GAL condition (miR-129-3p, miR-146b-5p, miR-543 and miR-550a) regulated multiple genes (e.g. NGEF, NOVA1, PDE5A) that show region- and age-specific transcription in the human brain [33], suggesting that deregulation of these transcripts might have significant consequences on brain function, and should be examined in the context of neurodevelopmental disorders. Thus, dermal fibroblasts are appealing, readily available human biomaterials, allowing to perform controlled, functional and reproducible experiments on human tissue. This is especially important for studying major, polygenic psychiatric disorders, where postmortem tissue availability is limited and the genetic animal models do not fully recapitulate the human disease. This study defined the miRNA/mRNA transcriptome responses of fibroblasts to metabolic stress in healthy individuals, and similar studies should be performed in diseased patient material - the response of their transcriptome to the metabolic stress will be more revealing of pathophysiology than the case-control differences under resting, unchallenged conditions.

\section{Supplementary Material}

Refer to Web version on PubMed Central for supplementary material. 


\section{Acknowledgments}

KM's work is supported by NIMH grants R01MH067234 and R01 MH079299. RS work was supported by NIMH grants MH01741, MH52339, and MH073630. We are grateful for Dr. Martin J. Schmidt for providing valuable comments and editing the manuscript.

\section{REFERENCES}

1. Horvath S, Janka Z, Mirnics K. Analyzing schizophrenia by DNA microarrays. Biol Psychiatry. 2010; 69:157-162. [PubMed: 20801428]

2. Mirnics K, Lewis DA. Genes and subtypes of schizophrenia. Trends Mol Med. 2001; 7:281-283. [PubMed: 11425621]

3. Glorioso C, Sabatini M, Unger T, Hashimoto T, Monteggia LM, et al. Specificity and timing of neocortical transcriptome changes in response to BDNF gene ablation during embryogenesis or adulthood. Mol Psychiatry. 2006; 11:633-648. [PubMed: 16702976]

4. Garbett KA, Horvath S, Ebert PJ, Schmidt MJ, Lwin K, et al. Novel animal models for studying complex brain disorders: BAC-driven miRNA-mediated in vivo silencing of gene expression. Mol Psychiatry. 2010 10.1038/mp.2010.1031.

5. Garbett KA, Hsiao EY, Kalman S, Patterson PH, Mirnics K. Effects of maternal immune activation on gene expression patterns in the fetal brain. Transl Psychiatry. 2012; 2:e98. [PubMed: 22832908]

6. Akin D, Manier DH, Sanders-Bush E, Shelton RC. Signal transduction abnormalities in melancholic depression. Int J Neuropsychopharmacol. 2005; 8:5-16. [PubMed: 15500705]

7. Gibson SA, Korade Z, Shelton RC. Oxidative stress and glutathione response in tissue cultures from persons with major depression. J Psychiatr Res. 2012; 46:1326-1332. [PubMed: 22841833]

8. de Nadal E, Ammerer G, Posas F. Controlling gene expression in response to stress. Nat Rev Genet. 2011; 12:833-845. [PubMed: 22048664]

9. Marroquin LD, Hynes J, Dykens JA, Jamieson JD, Will Y. Circumventing the Crabtree effect: replacing media glucose with galactose increases susceptibility of HepG2 cells to mitochondrial toxicants. Toxicol Sci. 2007; 97:539-547. [PubMed: 17361016]

10. Jacobs RA, Sly WS, Majerus PW. The regulation of fatty acid biosynthesis in human skin fibroblasts. J Biol Chem. 1973; 248:1268-1276. [PubMed: 4734469]

11. Raff RA. Induction of fatty acid synthesis in cultured mammalian cells: effects of cycloheximide and X-rays. J Cell Physiol. 1970; 75:341-351. [PubMed: 5449698]

12. Bensaude I, Callahan J, Philippart M. Fabry's disease as an -galactosidosis: evidence for an configuration in trihexosyl ceramide. Biochem Biophys Res Commun. 1971; 43:913-918. [PubMed: 5563756]

13. Malan-Muller S, Hemmings SM, Seedat S. Big effects of small RNAs: a review of microRNAs in anxiety. Mol Neurobiol. 2012; 47:726-739. [PubMed: 23150170]

14. Inui M, Martello G, Piccolo S. MicroRNA control of signal transduction. Nat Rev Mol Cell Biol. 2010; 11:252-263. [PubMed: 20216554]

15. Manier DH, Eiring A, Shelton RC, Sulser F. Beta-adrenoceptor-linked protein kinase A (PKA) activity in human fibroblasts from normal subjects and from patients with major depression. Neuropsychopharmacology. 1996; 15:555-561. [PubMed: 8946429]

16. APA. American Psychiatric Association; Washington, DC: 2000. Diagnostic and statistical manual of mental disorders.

17. Unger T, Korade Z, Lazarov O, Terrano D, Sisodia SS, Mirnics K. True and false discovery in DNA microarray experiments: transcriptome changes in the hippocampus of presenilin 1 mutant mice. Methods. 2005; 37(3):261-73. [PubMed: 16308155]

18. Mirnics K. Microarrays in Brain Research: Data Quality and Limitations Revisited. Current Genomics. 2006; 7:11-17.

19. Reich M, Liefeld T, Gould J, Lerner J, Tamayo P, et al. GenePattern 2.0. Nat Genet. 2006; 38:500501. [PubMed: 16642009]

20. Subramanian A, Kuehn H, Gould J, Tamayo P, Mesirov JP. GSEA-P: a desktop application for Gene Set Enrichment Analysis. Bioinformatics. 2007; 23:3251-3253. [PubMed: 17644558] 
21. Wang X. miRDB: a microRNA target prediction and functional annotation database with a wiki interface. RNA. 2008; 14:1012-1017. [PubMed: 18426918]

22. Tsai KW, Wu CW, Hu LY, Li SC, Liao YL, et al. Epigenetic regulation of miR-34b and miR-129 expression in gastric cancer. Int J Cancer. 2011; 129:2600-2610. [PubMed: 21960261]

23. Cao J, Shen Y, Zhu L, Xu Y, Zhou Y, et al. miR-129-3p controls cilia assembly by regulating CP110 and actin dynamics. Nat Cell Biol. 2012; 14:697-706. [PubMed: 22684256]

24. Kutty RK, Nagineni CN, Samuel W, Vijayasarathy C, Jaworski C, et al. Differential regulation of microRNA-146a and microRNA-146b-5p in human retinal pigment epithelial cells by interleukin-1beta, tumor necrosis factor-alpha, and interferon-gamma. Mol Vis. 2013; 19:737-750. [PubMed: 23592910]

25. Takahashi N, Nakaoka T, Yamashita N. Profiling of immune-related microRNA expression in human cord blood and adult peripheral blood cells upon proinflammatory stimulation. Eur J Haematol. 2012; 88:31-38. [PubMed: 21913990]

26. Hulsmans M, Van Dooren E, Mathieu C, Holvoet P. Decrease of miR-146b-5p in monocytes during obesity is associated with loss of the anti-inflammatory but not insulin signaling action of adiponectin. PLoS One. 2012; 7:e32794. [PubMed: 22393448]

27. Geraldo MV, Yamashita AS, Kimura ET. MicroRNA miR-146b-5p regulates signal transduction of TGF-beta by repressing SMAD4 in thyroid cancer. Oncogene. 2011; 31:1910-1922. [PubMed: 21874046]

28. Garcia, Al; Buisson, M.; Bertrand, P.; Rimokh, R.; Rouleau, E., et al. Down-regulation of BRCA1 expression by miR-146a and miR-146b-5p in triple negative sporadic breast cancers. EMBO Mol Med. 2011; 3:279-290. [PubMed: 21472990]

29. Katakowski M, Zheng X, Jiang F, Rogers T, Szalad A, et al. MiR-146b-5p suppresses EGFR expression and reduces in vitro migration and invasion of glioma. Cancer Invest. 2010; 28:10241030. [PubMed: 20874002]

30. Michel M, Schmidt MJ, Mirnics K. Immune system gene dysregulation in autism and schizophrenia. Dev Neurobiol. 2012; 72:1277-1287. [PubMed: 22753382]

31. Naydenov AV, MacDonald ML, Ongur D, Konradi C. Differences in lymphocyte electron transport gene expression levels between subjects with bipolar disorder and normal controls in response to glucose deprivation stress. Arch Gen Psychiatry. 2007; 64:555-564. [PubMed: 17485607]

32. Manier DH, Shelton RC, Ellis TC, Peterson CS, Eiring A, et al. Human fibroblasts as a relevant model to study signal transduction in affective disorders. J Affect Disord. 2000; 61:51-58. [PubMed: 11099740]

33. Kang HJ, Kawasawa Yl, Cheng F, Zhu Y, Xu X, Li M, Sousa AM, Pletikos M, Meyer KA, Sedmak G, Guennel T, Shin Y, Johnson MB, Krsnik Z, Mayer S, Fertuzinhos S, Umlauf S, Lisgo SN, Vortmeyer A, Weinberger DR, Mane S, Hyde TM, Huttner A, Reimers M, Kleinman JE, Sestan N. Spatio-temporal transcriptome of the human brain. Nature. 2011; 478(7370):483-9. [PubMed: 22031440] 


\section{Research highlights}

- metabolic stress produced strong mRNA and miRNA changes in human fibroblasts

- miRNA changes across two metabolic stress conditions showed substantial similarity

- $\quad$ miRNA expression contributed to altered mRNA expression

- $\quad$ miRNA and mRNA changes were correlated

- $\quad$ miR 129 3p, miR 146b 5p, miR 543 and miR 550a are mediators of metabolic stress 


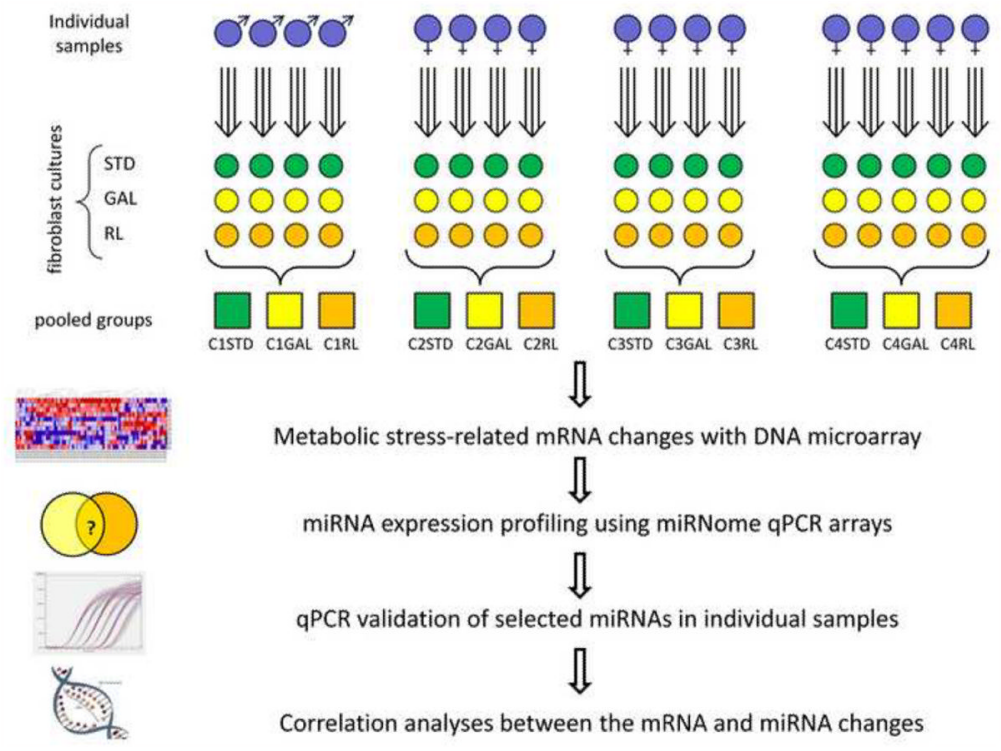

Figure 1. Experimental design.

Flowchart representing the sample collection and grouping, the experiments and analyses performed. In summary, skin biopsies were collected from 17 male and female individuals, and used to establish in vitro fibroblast cultures. Each fibroblast culture was then cultured in standard (STD), galactose containing (GAL) or reduced lipid (RL) media. DNA microarrays were used to detect the mRNA profile of each individual sample in each culture condition. 4-5 samples of similar age and gender were grouped before performing miRNA expression profiling with miRNome qPCR arrays. Grouping-sample effects were examined in individual samples with custom qPCR arrays containing selected miRNA assays. Finally, we preformed correlation analyses between the commonly changed miRNAs and their putative mRNA targets. 


\section{miRNAs are affected}

by GAL and RL treatment

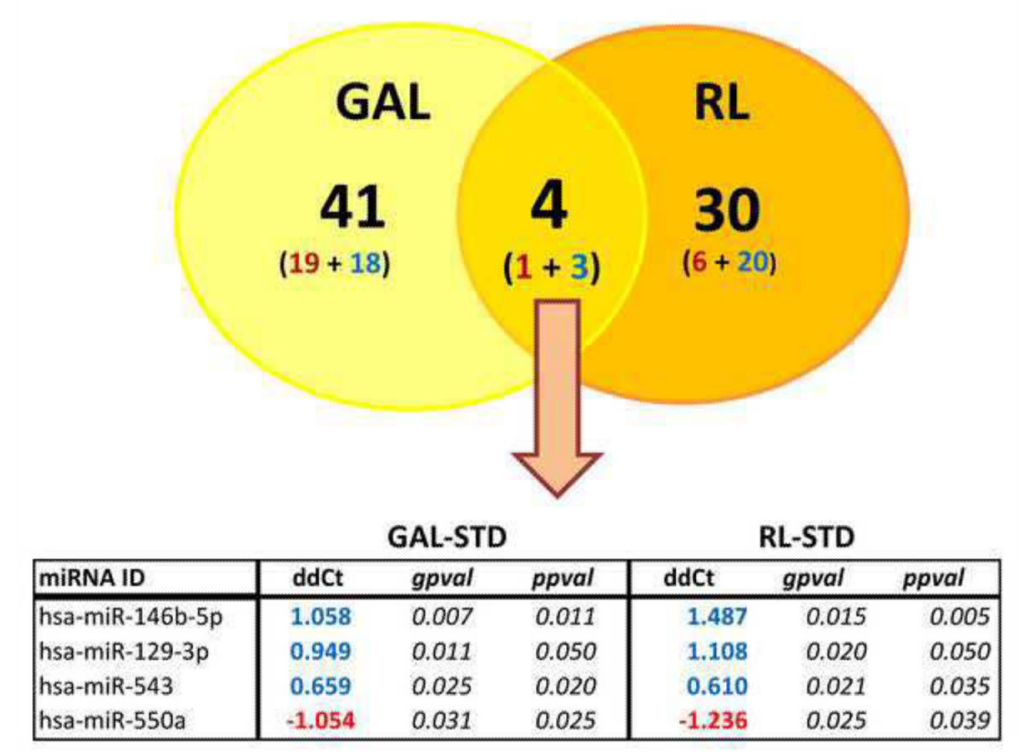

Figure 2. qPCR validation of selected mRNA expression changes.

10 transcripts were validated using qPCR (SCD, FABP3, DHCR7, INSIG1, QPRT, HMGCR, HMGCS1, LDLR, FADS1, FADS2). X- axis denotes microarray-obtained average $\log 2$ ratios (ALR), while $\mathrm{Y}$-axis denotes actin-normalized $-\Delta \Delta \mathrm{Ct}$ values obtained by qPCR. Note that the microarray and qPCR data were highly correlated (GAL: $r=0.92$ $\mathrm{p}<0.001$; RL: $r=0.72, \mathrm{p}<0.001$ ). For further details, see Supplemental Material 4. 

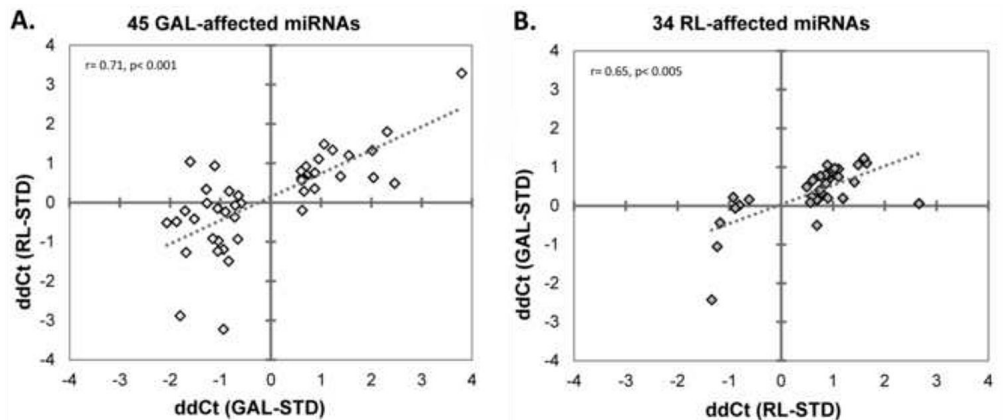

Figure 3. Commonly changed miRNAs in GAL and RL culture conditions.

45 and 34 miRNAs showed significant change in GAL and RL conditions, respectively (reddecreased; blue - increased). Note that the 4 commonly changed miRNAs had the same directionality in both GAL and RL conditions, and they were significantly altered in both pairwise (ppval) and groupwise (gpval) testing. 


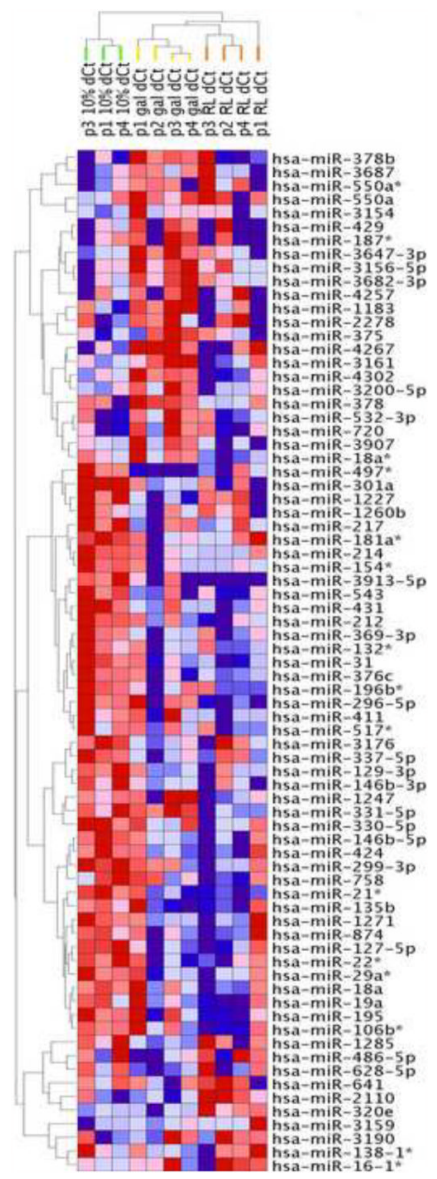

Figure 4. Overall miRNA pattern changes in GAL and RL conditions are highly correlated. The 45 miRNAs with significantly changed expression pattern in GAL conditions were assessed in the RL samples (A) and the 34 miRNAs with significantly changed expression pattern in RL conditions were assessed in the GAL samples (B). Note that the miRNA pattern observed in either metabolic stressor also showed a similar, highly correlated expression pattern in the other condition. 

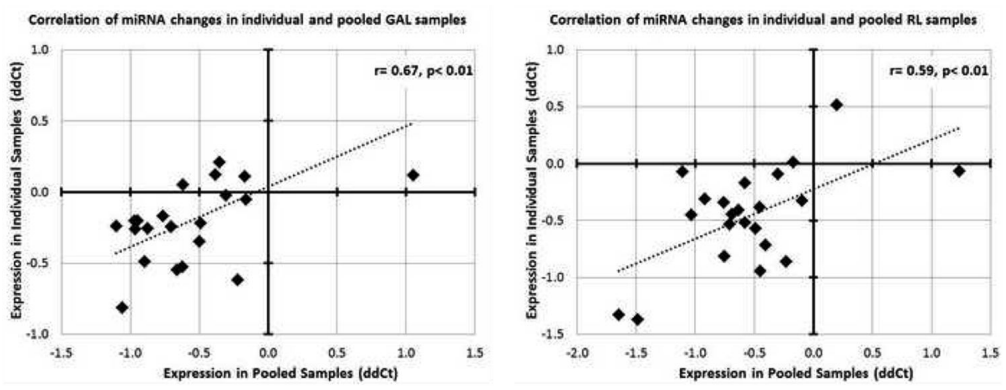

Figure 5. miRNA expression pattern separates the STD, GAL and RL lipid conditions.

Two-way unsupervised hierarchical clustering was performed on the expression level $(\Delta \mathrm{Ct})$ of 75 differentially expressed miRNAs. Samples were clustered vertically, miRNAs were clustered horizontally. Each colored square represents a normalized gene expression level, color coded for increase (red) or decrease (blue) from the mean. Color intensity is proportional to magnitude of change. The clustering resulted in a perfect separation of samples into three discrete groups corresponding to culture conditions (vertical dendrogram, green STD, yellow GAL, and orange RL). 

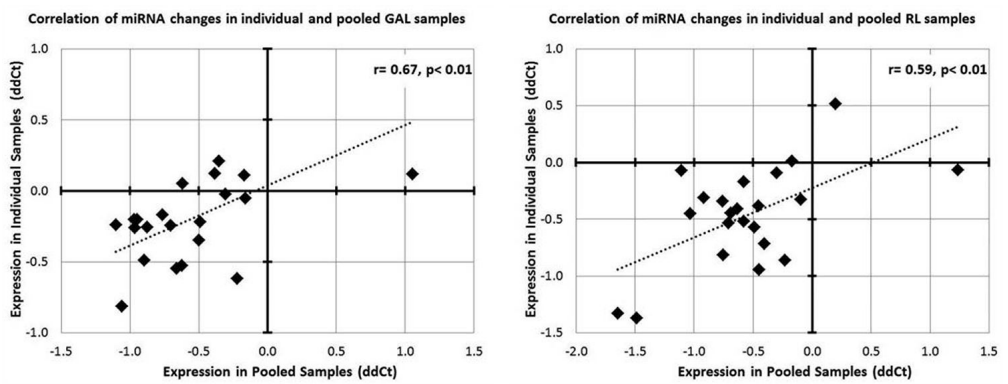

Figure 6. Correlation of miRNA changes between individual and pooled fibroblast samples cultured in GAL (A) and RL (B) conditions.

22 miRNAs were assessed in the validation study, with the pooled $\Delta \Delta \mathrm{CT}$ s plotted on $\mathrm{X}$ axis and the $\Delta \Delta \mathrm{CT}$ s from individual samples plotted on the $\mathrm{Y}$ axis. Each miRNA represents a single data point. The correlation coefficient indicates that pooling of the samples did not skew artificially the miRNA pattern observed in GAL and RL. 


\section{Table 1}

mRNA transcriptome changes. Microarray gene expression data, subjected to BioCarta pathway analysis with GSEA, identified 19 pathways enriched in fibroblasts cultured in GAL (A) and 15 in RL (B). The significantly changed BioCarta pathways are ranked by a net enrichment score (NES). The number of genes in each pathway is depicted in the Size column. Note that there are 4 differentially expressed pathways common for GAL and RL treatment $(*)$.

\begin{tabular}{|c|c|c|c|}
\hline \multicolumn{4}{|c|}{ A. Galactose (GAL) media } \\
\hline NAME & SIZE & NES & p-value \\
\hline RACCYCD $^{*}$ & 26 & 1.93 & 0.000000 \\
\hline CELLCYCLE & 23 & 1.83 & 0.000000 \\
\hline SRCRPTP & 11 & 1.70 & 0.000000 \\
\hline P53 & 16 & 1.70 & 0.002045 \\
\hline TEL & 18 & 1.73 & 0.010730 \\
\hline STATHMIN & 19 & 1.57 & 0.011516 \\
\hline CFTR & 12 & 1.65 & 0.012000 \\
\hline CERAMIDE & 22 & 1.67 & 0.012448 \\
\hline ATRBRCA & 21 & 1.60 & 0.016129 \\
\hline G2 & 24 & 1.59 & 0.026157 \\
\hline HSP27* & 15 & 1.47 & 0.026639 \\
\hline CARM_ER & 34 & 1.61 & 0.027451 \\
\hline PLCE & 12 & 1.47 & 0.030864 \\
\hline CHREBP2* & 42 & 1.61 & 0.031373 \\
\hline AKAPCENTROSOME & 15 & 1.53 & 0.031809 \\
\hline CHEMICAL & 22 & 1.56 & 0.032520 \\
\hline $\mathrm{RB}$ & 13 & 1.53 & 0.034137 \\
\hline PPARA* & 56 & 1.43 & 0.036000 \\
\hline ATM & 20 & 1.58 & 0.044807 \\
\hline
\end{tabular}

B. Reduced lipid (RL) media

\begin{tabular}{|l|c|c|c|}
\hline NAME & SIZE & NES & p-value \\
\hline PPARA $^{*}$ & 56 & $\mathbf{1 . 8 0}$ & 0.000000 \\
\hline P38MAPK & 39 & $\mathbf{1 . 5 6}$ & 0.009579 \\
\hline VIP & 26 & $\mathbf{1 . 6 0}$ & 0.009709 \\
\hline BAD & 26 & $\mathbf{1 . 4 8}$ & 0.015936 \\
\hline CHREBP2 $^{*}$ & 42 & $\mathbf{1 . 6 4}$ & 0.022945 \\
\hline RACCYCD $^{*}$ & 26 & $\mathbf{1 . 6 5}$ & 0.027559 \\
\hline GCR $^{*}$ & 19 & $\mathbf{1 . 4 6}$ & 0.038000 \\
\hline HSP27 $^{*}$ & 15 & $\mathbf{1 . 4 9}$ & 0.040541 \\
\hline
\end{tabular}




\begin{tabular}{|l|c|c|c|}
\hline NAME & SIZE & NES & p-value \\
\hline CD40 & 15 & $\mathbf{1 . 5 8}$ & 0.044000 \\
\hline LYM & 11 & $\mathbf{1 . 3 7}$ & 0.047714 \\
\hline TNFR2 & 18 & $\mathbf{1 . 5 3}$ & 0.048583 \\
\hline TALL1 & 15 & $\mathbf{1 . 5 2}$ & 0.048638 \\
\hline IL22BP & 16 & $\mathbf{- 1 . 5 6}$ & 0.020638 \\
\hline DNAFRAGMENT & 10 & $\mathbf{- 1 . 5 1}$ & 0.030488 \\
\hline AHSP & 11 & $\mathbf{- 1 . 5 9}$ & 0.043222 \\
\hline
\end{tabular}

* Commonly upregulated in NL and GAL treatment 
Table 2

miRNA expression profile changes in GAL (A) and RL (B) treatment in human fibroblasts. Blue increased expression; Red - reduced expression; $\Delta \Delta \mathrm{Ct}$ - normalized threshold cycle difference between experimental and control samples; gt - groupwise t-test; $\mathrm{pt}$ - pairwise t-test. The correlations of these data are graphically represented in Figure 3.

GAL-induced miRNA expression changes and their expression in RL

\begin{tabular}{|c|c|c|c|c|c|c|}
\hline miRNA ID & $\operatorname{ddCt}($ GAL-STD) & gt-test (GAL-STD) & pt-test $(G A L-S T D)$ & ddCt (RL-STD) & gt-test (RL-STD) & pt-test (RL-STD) \\
\hline hsa-miR-378b & -2.065 & 0.006 & 0.003 & -0.512 & 0.548 & 0.614 \\
\hline hsa-miR-1183 & -1.875 & 0.023 & 0.064 & -0.485 & 0.557 & N/A \\
\hline hsa-miR-429 & -1.801 & 0.019 & 0.154 & -2.877 & N/A & N/A \\
\hline hsa-miR-3682-3p & -1.704 & 0.031 & 0.178 & -0.212 & 0.659 & 0.936 \\
\hline hsa-miR-187* & -1.683 & 0.034 & 0.074 & -1.270 & N/A & N/A \\
\hline hsa-miR-4302 & -1.604 & 0.019 & 0.036 & 1.038 & 0.143 & 0.067 \\
\hline hsa-miR-375 & -1.522 & 0.149 & 0.041 & -0.417 & 0.535 & 0.862 \\
\hline hsa-miR-3200-5p & -1.283 & 0.041 & 0.128 & 0.338 & 0.489 & 0.509 \\
\hline hsa-miR-3161 & -1.269 & 0.017 & 0.002 & -0.015 & 0.982 & 0.865 \\
\hline hsa-miR-4267 & -1.154 & 0.006 & 0.015 & -0.915 & 0.105 & 0.085 \\
\hline hsa-miR-378 & -1.115 & 0.057 & 0.040 & 0.935 & 0.169 & 0.183 \\
\hline hsa-miR-3156-5p & -1.055 & 0.022 & 0.034 & -0.148 & 0.723 & 0.870 \\
\hline hsa-miR-550a & -1.054 & 0.031 & 0.025 & -1.236 & 0.025 & 0.039 \\
\hline hsa-miR-4257 & -1.044 & 0.020 & 0.327 & -0.972 & 0.227 & 0.436 \\
\hline hsa-miR-3159 & -0.943 & 0.142 & 0.037 & -3.222 & 0.299 & 0.325 \\
\hline hsa-miR-550a* & -0.941 & 0.042 & 0.133 & -1.192 & 0.130 & 0.072 \\
\hline hsa-miR-720 & -0.903 & 0.090 & 0.023 & -0.236 & 0.588 & 0.335 \\
\hline hsa-miR-2278 & -0.839 & 0.287 & 0.035 & -1.490 & 0.155 & 0.169 \\
\hline hsa-miR-3907 & -0.827 & 0.070 & 0.013 & 0.291 & 0.436 & 0.343 \\
\hline hsa-miR-3687 & -0.717 & 0.042 & 0.011 & -0.370 & 0.567 & 0.648 \\
\hline hsa-miR-532-3p & -0.709 & 0.062 & 0.029 & -0.069 & 0.820 & 0.757 \\
\hline hsa-miR-3190 & -0.658 & 0.430 & 0.050 & -0.930 & 0.242 & 0.572 \\
\hline hsa-miR-1247 & -0.645 & 0.450 & 0.022 & 0.177 & 0.803 & 0.800 \\
\hline hsa-miR-3647-3p & -0.590 & 0.011 & 0.048 & -0.017 & 0.949 & 0.960 \\
\hline hsa-miR-301a & 0.602 & 0.038 & 0.025 & 0.802 & 0.070 & 0.060 \\
\hline hsa-miR-369-3p & 0.612 & 0.029 & 0.030 & 0.575 & 0.052 & 0.025 \\
\hline hsa-miR-874 & 0.616 & 0.313 & 0.002 & 0.632 & 0.398 & 0.277 \\
\hline hsa-miR-486-5p & 0.626 & 0.279 & 0.029 & -0.196 & 0.754 & 0.680 \\
\hline hsa-miR-543 & 0.659 & 0.025 & 0.020 & 0.610 & 0.021 & 0.035 \\
\hline hsa-miR-1260b & 0.660 & 0.077 & 0.049 & 0.290 & 0.459 & 0.431 \\
\hline hsa-miR-424 & 0.698 & 0.061 & 0.011 & 0.919 & 0.104 & 0.052 \\
\hline hsa-miR-127-5p & 0.713 & 0.069 & 0.045 & 0.696 & 0.120 & 0.114 \\
\hline hsa-miR-3913-5p & 0.757 & 0.343 & 0.031 & N/A & N/A & N/A \\
\hline
\end{tabular}




\begin{tabular}{|c|c|c|c|c|c|c|}
\hline miRNA ID & ddCt (GAL-STD) & gt-test (GAL-STD) & pt-test (GAL-STD) & $\operatorname{ddCt}($ RL-STD) & gt-test (RL-STD) & pt-test $(R L-S T D)$ \\
\hline hsa-miR-628-5p & 0.871 & 0.020 & 0.067 & 0.355 & 0.440 & 0.552 \\
\hline hsa-miR-181a* & 0.876 & 0.039 & 0.014 & 0.759 & 0.213 & 0.146 \\
\hline hsa-miR-129-3p & 0.949 & 0.011 & 0.053 & 1.108 & 0.020 & 0.051 \\
\hline hsa-miR-146b-5p & 1.058 & 0.007 & 0.011 & 1.487 & 0.015 & 0.005 \\
\hline hsa-miR-1271 & 1.227 & 0.032 & 0.113 & 1.338 & 0.171 & 0.339 \\
\hline hsa-miR-1285 & 1.386 & 0.010 & 0.037 & 0.667 & 0.407 & 0.517 \\
\hline hsa-miR-337-5p & 1.550 & 0.017 & 0.103 & 1.200 & 0.060 & 0.061 \\
\hline hsa-miR-217 & 2.018 & 0.112 & 0.013 & 1.323 & 0.097 & 0.223 \\
\hline hsa-miR-3176 & 2.041 & 0.018 & 0.009 & 0.635 & 0.373 & 0.515 \\
\hline hsa-miR-497* & 2.313 & 0.040 & 0.079 & 1.803 & 0.098 & 0.204 \\
\hline hsa-miR-1227 & 2.462 & 0.014 & 0.113 & 0.488 & 0.343 & 0.172 \\
\hline hsa-miR-146b-3p & 3.795 & 0.046 & 0.123 & 3.291 & 0.216 & 0.205 \\
\hline
\end{tabular}

RL-induced miRNA expression changes and their expression in GAL

\begin{tabular}{|c|c|c|c|c|c|c|}
\hline miRNA ID & ddCt (RL-STD) & gt-test (RL-STD) & pt-test (RL-STD) & $\operatorname{ddCt}($ GAL-STD) & gt-test (GAL-STD) & pt-test $(G A L-S T D)$ \\
\hline hsa-miR-3154 & -1.342 & 0.039 & 0.047 & -2.425 & 0.161 & 0.267 \\
\hline hsa-miR-550a & -1.236 & 0.025 & 0.039 & -1.054 & 0.031 & 0.025 \\
\hline hsa-miR-16-1* & -1.181 & 0.014 & 0.063 & -0.437 & 0.347 & 0.311 \\
\hline hsa-miR-138-1* & -0.924 & 0.326 & 0.021 & 0.219 & 0.775 & 0.712 \\
\hline hsa-miR-641 & -0.887 & 0.042 & 0.253 & -0.059 & 0.875 & 0.726 \\
\hline hsa-miR-2110 & -0.805 & 0.043 & 0.031 & 0.017 & 0.953 & 0.965 \\
\hline hsa-miR-320e & -0.618 & 0.032 & 0.118 & 0.162 & 0.384 & 0.513 \\
\hline hsa-miR-376c & 0.493 & 0.039 & 0.027 & 0.490 & 0.114 & 0.050 \\
\hline hsa-miR-296-5p & 0.561 & 0.011 & 0.018 & 0.085 & 0.736 & 0.623 \\
\hline hsa-miR-517* & 0.595 & 0.099 & 0.013 & 0.245 & 0.475 & 0.375 \\
\hline hsa-miR-543 & 0.610 & 0.021 & 0.035 & 0.659 & 0.025 & 0.020 \\
\hline hsa-miR-214 & 0.636 & 0.006 & 0.032 & 0.703 & 0.088 & 0.075 \\
\hline hsa-miR-195 & 0.689 & 0.011 & 0.061 & 0.163 & 0.600 & 0.586 \\
\hline hsa-miR-18a* & 0.692 & 0.057 & 0.014 & -0.504 & 0.238 & 0.141 \\
\hline hsa-miR-19a & 0.710 & 0.103 & 0.035 & 0.168 & 0.660 & 0.323 \\
\hline hsa-miR-212 & 0.742 & 0.031 & 0.032 & 0.404 & 0.155 & 0.078 \\
\hline hsa-miR-132* & 0.753 & 0.120 & 0.002 & 0.766 & 0.122 & 0.292 \\
\hline hsa-miR-411 & 0.779 & 0.016 & 0.066 & 0.331 & 0.391 & 0.410 \\
\hline hsa-miR-330-5p & 0.854 & 0.117 & 0.007 & 0.797 & 0.261 & 0.347 \\
\hline hsa-miR-431 & 0.864 & 0.023 & 0.004 & 0.579 & 0.063 & 0.016 \\
\hline hsa-miR-154* & 0.891 & 0.008 & 0.115 & 1.052 & 0.087 & 0.110 \\
\hline hsa-miR-18a & 0.900 & 0.039 & 0.029 & 0.198 & 0.597 & 0.375 \\
\hline hsa-miR-29a* & 0.912 & 0.041 & 0.116 & 0.801 & 0.085 & 0.220 \\
\hline hsa-miR-135b & 0.927 & 0.148 & 0.037 & 0.698 & 0.395 & 0.249 \\
\hline hsa-miR-22* & 1.033 & 0.049 & 0.194 & 0.966 & 0.083 & 0.339 \\
\hline
\end{tabular}




\begin{tabular}{|c|c|c|c|c|c|c|}
\hline miRNA ID & ddCt (RL-STD) & gt-test $(R L-S T D)$ & pt-test (RL-STD) & ddCt (GAL-STD) & gt-test (GAL-STD) & pt-test (GAL-STD) \\
\hline hsa-miR-31 & 1.105 & 0.004 & 0.012 & 0.750 & 0.065 & 0.079 \\
\hline hsa-miR-129-3p & 1.108 & 0.020 & 0.051 & 0.949 & 0.011 & 0.053 \\
\hline hsa-miR-758 & 1.188 & 0.042 & 0.042 & 0.199 & 0.672 & 0.608 \\
\hline hsa-miR-106b* & 1.193 & 0.035 & 0.229 & 0.188 & 0.795 & 0.918 \\
\hline hsa-miR-299-3p & 1.414 & 0.048 & 0.109 & 0.612 & 0.159 & 0.243 \\
\hline hsa-miR-146b-5p & 1.487 & 0.015 & 0.005 & 1.058 & 0.007 & 0.011 \\
\hline hsa-miR-196b* & 1.599 & 0.025 & 0.034 & 1.226 & 0.114 & 0.289 \\
\hline hsa-miR-21* & 1.647 & 0.003 & 0.019 & 1.102 & 0.093 & 0.052 \\
\hline hsa-miR-331-5p & 2.660 & 0.010 & 0.041 & 0.058 & 0.915 & 0.932 \\
\hline
\end{tabular}


Table 3

miRNA and mRNA expression changes are correlated in human fibroblasts. The three commonly changed miRNAs in both GAL and RL conditions were correlated with expression changes of 52 putative target mRNAs. A. miR-129-3p target changes, B. miR-550a target changes, C. miR-146b-5p target changes. The commonly changed mRNAs in both GAL- and RL-medium exposed human fibroblasts are marked with an asterisk.

\begin{tabular}{|c|c|c|c|c|c|}
\hline \multicolumn{6}{|c|}{ A. miR-129-3p } \\
\hline Gene & ALR GAL & pval GAL & Gene & ALR RL & pval RL \\
\hline FADS $1 *$ & 1.08 & 0.000001 & FADS $1 *$ & 1.22 & 0.000000 \\
\hline FADS $1 *$ & 1.08 & 0.000001 & FADS $1 *$ & 1.22 & 0.000000 \\
\hline FADS $1 *$ & 1.06 & 0.000001 & FADS $1 *$ & 1.11 & 0.000000 \\
\hline DGKI & 0.68 & 0.000073 & STARD4 & 0.94 & 0.000000 \\
\hline LHFPL2 & 0.63 & 0.000000 & TMTC1 ${ }^{*}$ & 0.57 & 0.000173 \\
\hline PTGER4* & 0.51 & 0.000302 & PTGER4* & 0.57 & 0.000329 \\
\hline $\mathrm{TMTC}^{*}{ }^{*}$ & 0.50 & 0.000007 & CCDC102B & 0.51 & 0.006941 \\
\hline CDK1 & 0.49 & 0.007441 & RCAN2 & 0.45 & 0.000727 \\
\hline CDCA7L & 0.47 & 0.000002 & CHML & 0.43 & 0.000010 \\
\hline PKIA & 0.43 & 0.000075 & RFX7 & 0.41 & 0.000179 \\
\hline C2orf88 & 0.41 & 0.000036 & RIMS1 & 0.38 & 0.004726 \\
\hline \multirow[t]{3}{*}{ RDH10 } & -0.42 & 0.000011 & CYP27C1 & 0.38 & 0.000169 \\
\hline & & & ZNF436 & -0.42 & 0.000329 \\
\hline & & & BAALC & -0.60 & 0.000524 \\
\hline
\end{tabular}

B. miR-550a

\begin{tabular}{|c|c|c|c|c|c|}
\hline Gene & ALR GAL & pval GAL & Gene & ALR RL & pval RL \\
\hline CLIC2 & 1.18 & 0.000000 & DSTN & 0.44 & 0.000322 \\
\hline SPATA13 & 0.50 & 0.000059 & EXPH5 ${ }^{*}$ & 0.41 & 0.008081 \\
\hline C1QTNF2 & 0.48 & 0.002883 & NGEF & 0.40 & 0.005541 \\
\hline OSBPL8 & 0.48 & 0.000000 & APOL6 & -0.93 & 0.000000 \\
\hline PLD1 & 0.45 & 0.000245 & & & \\
\hline SCARA3 & 0.39 & 0.000014 & & & \\
\hline ADAMTS6 & -0.38 & 0.005308 & & & \\
\hline EXPH5 ${ }^{*}$ & -0.51 & 0.001573 & & & \\
\hline MYH2 & -0.80 & 0.000192 & & & \\
\hline
\end{tabular}

C. miR-146b-5p

\begin{tabular}{|cccccc|}
\hline Gene & ALR GAL & pval GAL & Gene & ALR RL & pval RL \\
\hline IFIT3 & $\mathbf{0 . 6 5}$ & 0.000003 & SVIL $^{*}$ & $\mathbf{0 . 5 8}$ & 0.000000 \\
IQGAP3 & $\mathbf{0 . 6 4}$ & 0.000058 & KRTAP1-5 $^{*}$ & $\mathbf{0 . 5 6}$ & 0.000110
\end{tabular}




\begin{tabular}{|c|c|c|c|c|c|}
\hline Gene & ALR GAL & pval GAL & Gene & ALR RL & pval $R L$ \\
\hline IRAK1 & 0.61 & 0.000001 & SVIL $^{*}$ & 0.50 & 0.000009 \\
\hline SYT14 & 0.61 & 0.000101 & CLCN6 ${ }^{*}$ & 0.46 & 0.000000 \\
\hline RAB7L1 & 0.58 & 0.000000 & PID1 & 0.42 & 0.000173 \\
\hline RAB7L1 & 0.55 & 0.000000 & NOVA1 & 0.41 & 0.000848 \\
\hline CLCN6* & 0.52 & 0.000001 & SLC1A1* & -0.45 & 0.000093 \\
\hline SVIL ${ }^{*}$ & 0.44 & 0.000079 & LRRC15 & -0.45 & 0.000372 \\
\hline STRBP & 0.43 & 0.000607 & KIAA1199 & -0.47 & 0.001176 \\
\hline TIMELESS & 0.42 & 0.000047 & & & \\
\hline RACGAP1 & 0.41 & 0.000632 & & & \\
\hline STIL & 0.40 & 0.002208 & & & \\
\hline WISP1 & -0.38 & 0.002183 & & & \\
\hline DNAL1 & -0.39 & 0.000081 & & & \\
\hline DNAL1 & -0.40 & 0.002178 & & & \\
\hline SLC1A1 ${ }^{*}$ & -0.49 & 0.000259 & & & \\
\hline KRTAP1-5* & -0.53 & 0.003753 & & & \\
\hline RASGRP1 & -0.67 & 0.000057 & & & \\
\hline
\end{tabular}

D. $\operatorname{miR-543}$

\begin{tabular}{|c|c|c|c|c|c|}
\hline Gene & ALR GAL & pval GAL & Gene & ALR RL & pval RL \\
\hline RRAGD* & 2.36 & 0.000000 & NRXN3 & 1.98 & 0.009901 \\
\hline RRAGD & 1.48 & 0.000000 & $\mathrm{ABCA} 1^{*}$ & 1.60 & 0.000000 \\
\hline PAG1 & 0.98 & 0.000000 & $\mathrm{ABCA} 1^{*}$ & 0.92 & 0.000000 \\
\hline PAG1 & 0.91 & 0.000000 & PLA2G4A & 0.86 & 0.002028 \\
\hline LAMA1 & 0.83 & 0.000029 & GALNT1 & 0.84 & 0.003950 \\
\hline C3orf55 & 0.82 & 0.000002 & RRAGD* & 0.79 & 0.009357 \\
\hline PAG1 ${ }^{*}$ & 0.80 & 0.000000 & PPP1R12B ${ }^{*}$ & 0.76 & 0.003190 \\
\hline PRKAR2B ${ }^{*}$ & 0.76 & 0.000002 & GALNT1 & 0.70 & 0.005351 \\
\hline TOX & 0.68 & 0.000073 & GALNT1 & 0.63 & 0.002321 \\
\hline ZNF367 & 0.64 & 0.000001 & COL16A1 & 0.61 & 0.000219 \\
\hline CDK1 & 0.63 & 0.000099 & ZNF436 & 0.56 & 0.000329 \\
\hline PBX3 & 0.62 & 0.000000 & TRIM13 & 0.50 & 0.000035 \\
\hline DCLK1 & 0.61 & 0.000864 & ZNF436 & 0.49 & 0.001606 \\
\hline FNIP2* & 0.58 & 0.000013 & DUSP ${ }^{*}$ & 0.46 & 0.000321 \\
\hline ZCCHC14 & 0.58 & 0.000000 & EVI5 & 0.39 & 0.000685 \\
\hline CDK1 & 0.58 & 0.000135 & TGFB1 & 0.39 & 0.000077 \\
\hline GTSE1 & 0.56 & 0.001911 & DDX3X & 0.38 & 0.005765 \\
\hline CDK1 & 0.56 & 0.000136 & PAG1 ${ }^{*}$ & 0.38 & 0.000839 \\
\hline BEX2 & 0.55 & 0.000005 & FNIP2 ${ }^{*}$ & 0.38 & 0.000458 \\
\hline GTSE1 & 0.54 & 0.001029 & CCDC117 & -0.38 & 0.000395 \\
\hline DUSP3 ${ }^{*}$ & 0.54 & 0.000000 & ADAMTSL1 & -0.41 & 0.000269 \\
\hline
\end{tabular}

Exp Cell Res. Author manuscript; available in PMC 2015 January 15. 


\begin{tabular}{|c|c|c|c|c|c|}
\hline Gene & ALR GAL & pval GAL & Gene & ALR RL & pval RL \\
\hline FNIP2 & 0.52 & 0.000006 & EEF1A1 & -0.42 & 0.003515 \\
\hline $\mathrm{ESCO} 2$ & 0.51 & 0.000066 & SMAD5 & -0.42 & 0.008163 \\
\hline E2F7 & 0.51 & 0.000144 & ADAMTSL1 * & -0.43 & 0.002492 \\
\hline FMNL2 & 0.49 & 0.000014 & TMEM133 & -0.43 & 0.003737 \\
\hline B3GNT5 & 0.49 & 0.000143 & ADAMTSL1 ${ }^{*}$ & -0.44 & 0.004797 \\
\hline $\mathrm{ABCA} 1^{*}$ & 0.49 & 0.000012 & PDP1 & -0.44 & 0.000010 \\
\hline CDK1 & 0.49 & 0.007441 & ADAMTSL1 & -0.53 & 0.000006 \\
\hline OSBPL8 & 0.48 & 0.000000 & CHML & -0.53 & 0.000279 \\
\hline DCLK1 & 0.48 & 0.003116 & PDE5A* & -0.56 & 0.002906 \\
\hline DCLK1 & 0.48 & 0.003116 & GBP1 & -0.60 & 0.003012 \\
\hline SLC2A13 & 0.47 & 0.000000 & RAPH1 ${ }^{*}$ & -0.68 & 0.000064 \\
\hline OSBPL1A & 0.47 & 0.000000 & HIPK2 & -0.71 & 0.000009 \\
\hline ABCA1 * & 0.47 & 0.000006 & PRKAR2B ${ }^{*}$ & -0.75 & 0.000159 \\
\hline RAD51AP1 & 0.46 & 0.000394 & CREB 5 * & -1.49 & 0.000041 \\
\hline DUSP3 & 0.46 & 0.000000 & CREB $5^{*}$ & -1.58 & 0.000760 \\
\hline LAMP2 & 0.45 & 0.000000 & & & \\
\hline GTSE1 & 0.45 & 0.003273 & & & \\
\hline FMNL2 & 0.45 & 0.000000 & & & \\
\hline WHSC1 & 0.44 & 0.000477 & & & \\
\hline STRBP & 0.43 & 0.000607 & & & \\
\hline $\mathrm{CCNF}$ & 0.43 & 0.000305 & & & \\
\hline SMC4 & 0.42 & 0.000009 & & & \\
\hline DUSP3 & 0.42 & 0.000000 & & & \\
\hline C3orf55 & 0.41 & 0.000024 & & & \\
\hline SLC2A13 & 0.40 & 0.000035 & & & \\
\hline ATP6V1A & 0.40 & 0.000000 & & & \\
\hline OSBPL8 & 0.40 & 0.000000 & & & \\
\hline HMGB2 & 0.40 & 0.000029 & & & \\
\hline ACYP1 & 0.40 & 0.000001 & & & \\
\hline RBM47 & 0.40 & 0.000154 & & & \\
\hline MEGF9 & 0.40 & 0.000002 & & & \\
\hline ZCCHC14 & 0.40 & 0.000657 & & & \\
\hline OSBPL1A & 0.40 & 0.000003 & & & \\
\hline CD163 & 0.39 & 0.005055 & & & \\
\hline ATP6V1A & 0.39 & 0.000022 & & & \\
\hline WHSC1 & 0.38 & 0.000535 & & & \\
\hline TMEM135 & 0.38 & 0.000045 & & & \\
\hline SC5DL & 0.38 & 0.000265 & & & \\
\hline NRXN3 & -0.38 & 0.002052 & & & \\
\hline USP53 & -0.38 & 0.002009 & & & \\
\hline NRCAM & -0.38 & 0.000730 & & & \\
\hline PDE5A* & -0.39 & 0.000332 & & & \\
\hline
\end{tabular}

Exp Cell Res. Author manuscript; available in PMC 2015 January 15. 


\begin{tabular}{|c|c|c|c|c|c|}
\hline Gene & ALR GAL & pval GAL & Gene & ALR RL & pval $R L$ \\
\hline MDM4 & -0.40 & 0.000436 & & & \\
\hline COL1A2 & -0.41 & 0.000018 & & & \\
\hline TRIM13 & -0.41 & 0.001256 & & & \\
\hline SNAPC3 & -0.43 & 0.000598 & & & \\
\hline ADAMTSL1 ${ }^{*}$ & -0.43 & 0.000002 & & & \\
\hline ARHGAP29 & -0.45 & 0.000223 & & & \\
\hline RAPH1 & -0.45 & 0.000001 & & & \\
\hline SYNE1 & -0.47 & 0.000020 & & & \\
\hline GATS & -0.47 & 0.000009 & & & \\
\hline RAPH1* & -0.49 & 0.000071 & & & \\
\hline CREB5* & -0.50 & 0.000029 & & & \\
\hline RAPH1 & -0.52 & 0.000001 & & & \\
\hline RAPH1 & -0.52 & 0.000007 & & & \\
\hline ACVR2A & -0.52 & 0.000006 & & & \\
\hline CHN1 & -0.53 & 0.000002 & & & \\
\hline FNDC3B & -0.54 & 0.000034 & & & \\
\hline CACNB4 & -0.56 & 0.000001 & & & \\
\hline SYTL4 & -0.56 & 0.000113 & & & \\
\hline ADAMTSL1 ${ }^{*}$ & -0.57 & 0.000000 & & & \\
\hline $\mathrm{CREB}^{*}{ }^{*}$ & -0.59 & 0.000234 & & & \\
\hline ADAMTSL1 & -0.60 & 0.000003 & & & \\
\hline PPP1R12B* & -0.63 & 0.000179 & & & \\
\hline PAWR & -0.64 & 0.000017 & & & \\
\hline ID4 & -0.74 & 0.000000 & & & \\
\hline ID4 & -0.80 & 0.000000 & & & \\
\hline ID4 & -0.83 & 0.000002 & & & \\
\hline ARRDC4 & -0.85 & 0.000002 & & & \\
\hline CYP26B1 & -1.16 & 0.000000 & & & \\
\hline
\end{tabular}

ALR- average $\log 2$ ratio; RL- reduced lipid media; GAL- galactose media * commonly changed mRNAs in GAL and NL treatment 


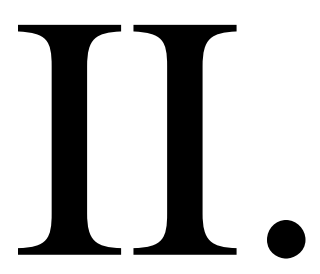




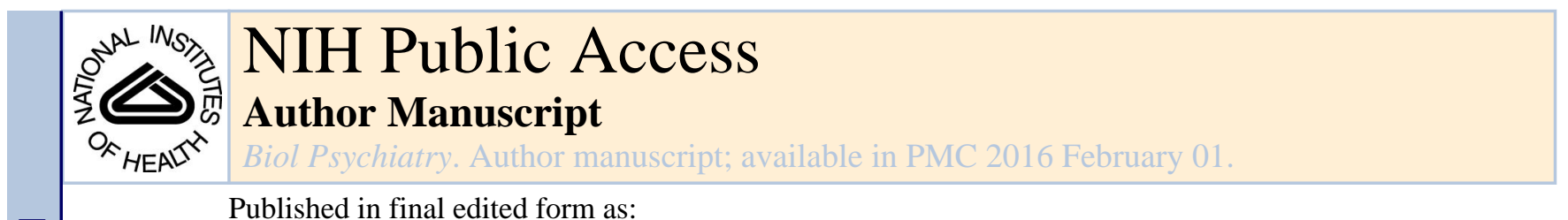

Published in final edited form as:

Biol Psychiatry. 2015 February 1; 77(3): 256-265. doi:10.1016/j.biopsych.2014.05.015.

\title{
Coordinated mRNA/miRNA changes in fibroblasts of patients with major depression
}

\author{
Krassimira A. Garbett ${ }^{1, \$}$, Andrea Vereczkei ${ }^{1,2, \$}$, Sára Kálmán ${ }^{1,3}$, Jacquelyn A Brown ${ }^{1}$, \\ Warren D. Taylor ${ }^{1}$, Gábor Faludi ${ }^{4}$, Željka Korade ${ }^{1,6}$, Richard C. Shelton ${ }^{5}$, and Károly \\ Mirnics ${ }^{1,3,6,{ }^{*}}$ \\ ${ }^{1}$ Department of Psychiatry, Vanderbilt University, Nashville, TN, USA \\ ${ }^{2}$ Institute for Medical Chemistry, Molecular Biology and Pathobiochemistry, Semmelweis \\ University, Budapest, Hungary \\ ${ }^{3}$ Department of Psychiatry, University of Szeged, Szeged, Hungary \\ ${ }^{4}$ Department of Psychiatry, Kútvölgyi Clinical Centre, Semmelweis University, Budapest, Hungary \\ ${ }^{5}$ Department of Psychiatry, University of Alabama, Birmingham, AL, USA \\ ${ }^{6}$ Vanderbilt Kennedy Center for Research on Human Development, Vanderbilt University, \\ Nashville, TN, USA
}

\section{Abstract}

Background-Peripheral biomarkers for major psychiatric disorders have been an elusive target for the last half a century. Dermal fibroblasts are a simple, relevant, and much underutilized model for studying molecular processes of patients with affective disorders as they share considerable similarity of signal transduction with neuronal tissue.

Methods-Cultured dermal fibroblast samples from patients with Major Depressive Disorder (MDD) and matched controls (CNTR) ( $\mathrm{n}=16$ pairs, 32 samples) were assayed for genome wide mRNA expression using microarrays. In addition, a simultaneous qPCR-based assessment of $>1,000$ miRNA species was performed. Finally, to test the relationship between the mRNAmiRNA expression changes, the two datasets were correlated with each other.

(C) 2014 Society of Biological Psychiatry. Published by Elsevier Inc. All rights reserved.

*Correspondence: Karoly Mirnics, Department of Psychiatry, Vanderbilt University, 8130A MRB III, 465 21st Avenue South, Nashville TN 37232, USA, karoly.mirnics@ vanderbilt.edu, Office phone: 615-936-1074, http://www.mirnicslab.org.

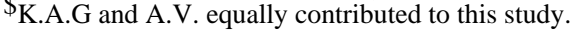

Financial Disclosures:

RCS receives unrelated research support from Assurex Health, Bristol Myers Squibb, Eli Lilly and Company, Elan Corp; Euthymics Bioscience; Forest Pharmaceuticals, Janssen Pharmaceutica, Jazz Pharmaceuticals, Naurex, Inc., Novartis Pharmaceuticals, Otsuka Pharmaceuticals, Pamlab, Inc., Pfizer, Inc., Repligen, Corp., Ridge Diagnostics, St. Jude Medical, Inc., Takeda Pharmaceuticals and consults for Bristol-Myers Squibb Company, Cerecor, Inc., Cyberonics, Inc., Eli Lilly and Company, Forest Pharmaceuticals, Janssen Pharmaceutica, Medtronic, Inc., Naurex, Inc., Pamlab, Inc., Pfizer, Inc., Ridge Diagnostics, Shire Plc, and Takeda Pharmaceuticals. All other authors report no biomedical financial interests or potential conflicts of interest.

Publisher's Disclaimer: This is a PDF file of an unedited manuscript that has been accepted for publication. As a service to our customers we are providing this early version of the manuscript. The manuscript will undergo copyediting, typesetting, and review of the resulting proof before it is published in its final citable form. Please note that during the production process errors may be discovered which could affect the content, and all legal disclaimers that apply to the journal pertain. 
Results-Our data revealed that MDD fibroblasts, when compared to matched controls, showed a strong mRNA gene expression pattern change in multiple molecular pathways, including cell-tocell communication, innate/adaptive immunity and cell proliferation. Furthermore, the same patient fibroblasts showed altered expression of a distinct panel of 38 miRNAs, which putatively targeted many of the differentially expressed mRNAs. The miRNA-mRNA expression changes appeared to be functionally connected, as the majority of the miRNA and mRNA changes were in the opposite direction.

Conclusions-Our data suggest that a combined miRNA-mRNA assessments are informative about the disease process, and that analyses of dermal fibroblasts might lead to the discovery of promising peripheral biomarkers of MDD, which could be potentially used to aid the diagnosis and allow mechanistic testing of disturbed molecular pathways.

\section{Keywords}

human fibroblasts; major depression; miRNA; gene expression; DNA microarray; biomarker

\section{INTRODUCTION}

There has been an intensive search for peripheral biomarkers of major psychiatric disorders for the last half a century. These efforts encompassed gene expression profiling of peripheral mononuclear cells $(1,2)$, biochemical evaluations of serum (3), urine (4), saliva (5), and cerebrospinal fluid (6), gene association studies of DNA markers (7) and many other approaches. More recently, inducible pluripotent stem cells (IPSCs) emerged as a very promising model for studying neuronal lineage disturbances across various disorders (8-10). Unfortunately, the complex diagnostic-phenotypic-genetic-etiologic heterogeneity continues (11) to provide significant obstacles for identifying highly specific and sensitive peripheral biomarkers of mental disorders.

Transcriptome profiling experiments of postmortem human brain tissue from subjects with major depressive disorder (MDD) suggest evidence of local inflammatory, apoptotic, oxidative stress and multiple other, non-brain specific molecular processes (12-17). Emerging evidence argues that many of these changes might be, at least partially, driven by altered expression of microRNAs (miRNAs): miRNA levels change during stress, in the brain of animal models of depression, and in human postmortem brain of MDD subjects(18) and depressed suicide subjects (19).

Importantly, peripheral biomarker studies of MDD over the last several decades revealed that peripheral growth factors, pro-inflammatory cytokines, endocrine factors, and metabolic markers all contribute to the disease pathophysiology (20). These combined data suggest that MDD is not only a disease of the CNS, but affects the whole body $(21,22)$, and that peripheral cellular-molecular events are strongly correlated with the disease pathology in the CNS (23). Experimental data suggest that analyzing patient dermal fibroblasts is a simple, relevant, and much underutilized model for studying processes of signal transduction in patients with affective disorders (24-26). Dermal fibroblasts are easy to establish and maintain in culture without transformation, and the majority of confounding factors (e.g. life style or medication use) are virtually eliminated after several rounds of cell division. 
Furthermore, a recent study of fibroblasts obtained from MDD patients highlights the role that oxidative stress might play in the pathophysiology of MDD (25), which has been already well-established across multiple other research models and patient populations (27, 28). Thus, analyzing dermal fibroblast cultures from patients can give us meaningful insights into the molecular effects of the combined genetic predisposition to the disorder.

It appears that biomarker panels hold a greater promise than single analyte molecules in aiding the diagnosis, monitoring disease progression or therapeutic response in MDD (20). As understanding non-neuronal changes in MDD can be informative of the overall disease pathophysiology (23), we performed a combined mRNA-miRNA profiling of dermal fibroblasts from patients with MDD and matched controls.

\section{METHODS AND MATERIALS}

\section{Participants in the study}

The Study was approved by the Vanderbilt University Institutional Review Board (IRB). Procedures for recruitment and diagnosis have been described previously $(24,25)$. All participants were diagnosed with a current Major Depressive Episode according to the Structured Clinical Interview for DSM-IV-TR (29) with an exclusion criteria of other primary axis I DSM-IV diagnosis. A written informed consent was obtained from all participants before collecting skin biopsy samples. Sixteen pairs of subjects with Major Depressive Disorder (MDD) and healthy controls (CNTR) were matched by age, race and sex. The average age of the MDD patients and CNTR were comparable (MDD=34.9, $\mathrm{CNTR}=35.2)$, as were the sex $(12 \mathrm{~F} / 4 \mathrm{M}$ in both groups) and race (12W/4AA), but there was a significant difference in body mass index at the time of skin biopsy (25.4 in CNTR and 32.3 in MDD, $\mathrm{p}=0.03$ ) (Supplemental Table S1).

\section{Human dermal fibroblasts}

The skin biopsy was obtained from the lateral side of the upper arm $(1 \times 2 \mathrm{~mm})$ according to a protocol previously described in details $(24,25)$. The sample was put into regular Dulbecco's Modified Eagle's Medium (DMEM, MediaTech, Manassas, VA, USA) without serum and processed the same day. Briefly, biopsy sample was cut into several smaller pieces with scissors and incubated in trypsin and collagenase mix at $37^{\circ} \mathrm{C}$ for $1 \mathrm{hr}$. Regular medium was added [DMEM containing high glucose, L-glutamine, 10\% fetal bovine serum (FBS, ThermoScientific HyClone, Logan, UT, USA), and Penicillin/Streptomycin solution (MediaTech)] and biopsy pieces with dissociated cells were pelleted by centrifugation. The supernatant was discarded. Biopsy pieces with cells were resuspended in fresh regular medium and transferred to $60 \mathrm{~mm}$ tissue culture plates. Cells were cultured in incubator at $37^{\circ} \mathrm{C}$ and $5 \% \mathrm{CO}_{2}$ concentration. Medium was changed 3 times a week. In about 2-3 weeks the fibroblasts divided and became confluent. The fibroblasts were subcultured using $0.5 \%$ Trypsin-EDTA (Invitrogen) as described elsewhere (30) and expanded for freezing in a liquid nitrogen cell repository or expanded for experiments. Selected fibroblasts from matching patient/control pairs were cultured simultaneously to ensure they grow under the same conditions. Cell growth and proliferation were checked regularly during the whole experiment. All cultured fibroblasts were less than passage 15. At the end of experiment, the 
fibroblasts were washed $2 \mathrm{X}$ with ice-cold PBS, collected with cell scraper, pelleted by centrifugation and frozen on dry ice and stored at $-80^{\circ} \mathrm{C}$.

mRNA expression analysis by microarrays

Total RNA was isolated using the $m i r V a n a^{\mathrm{TM}}$ miRNA Isolation Kit (Ambion, Foster City, CA, USA) and RNA quality assessed by an Agilent 2100 Bioanalyzer (Agilent, Palo Alto, California, USA). cDNA was generated using $2 \mu \mathrm{g}$ of total RNA. cDNA synthesis, amplification and labeling were performed using The Enzo Life Sciences Single-Round RNA Amplification and Biotin Labeling System (Enzo Life Sciences, Famingdale, NY, USA). $5 \mu \mathrm{g}$ of the biotin labeled, fragmented aRNA were hybridized to a GeneChip HT HGU133+ PM Array Plate (Affymetrix Inc, Santa Clara, CA, USA) at the Vanderbilt Microarray Shared Resource core facility.

Segmented images from each mircoarray were normalized and $\log _{2}$ transformed using GCrobust multi-array analysis (GC-RMA) (31). The normalized expression values were used in all analyses. Average expression values for each group (MDD and CNTR) were calculated for each gene probe. The magnitude of expression change was determined by the Average Logarithmic Ratio $\left(\mathrm{ALR}=\right.$ mean $_{\mathrm{MDD}}$ - mean $\left._{\mathrm{CNTR}}\right)$. Student's paired and grouped two-tailed t-tests were used to test the significance of the difference in gene expression (32-36). A gene was considered to be differentially expressed between MDD and CNTR when it met the dual criteria of $|\mathrm{ALR}|>0.585(50 \%)$ and both pairwise and groupwise $\mathrm{p}$-values $<0.05$.

The differentially expressed genes were subjected to a two-way hierarchical clustering analysis based on Pearson correlation using GenePattern software (37). Gene set enrichment analysis (GSEA) based on pre-defined gene classes were carried out with the GenePattern software (38). GSEA determines whether an a priori defined set of genes shows statistically significant, concordant differences between our subject groups based on the BioCarta defined molecular pathways. GSEA calculates a normalized enrichment score (NES), which reflects the degree to which a gene set is overrepresented in the ranked list of genes and a nominal p-value which estimates the statistical significance of the enrichment score for a single gene set. BioCarta gene sets were considered differentially expressed at nominal $p$ value $<0.05$.

\section{mRNA data validation by $\mathrm{qPCR}$}

cDNA was generated with random primers using High Capacity cDNA Reverse Transcription Kit (Applied Biosystems, Foster City, CA). Primers for 13 genes (heparinbinding EGF-like growth factor - HBEGF, major histocompatibility complex, class II invariant chain - CD74, major histocompatibility complex, class II, DP alpha 1 - HLADPA1, glutathione S-transferase theta 1 - GSTT1, major histocompatibility complex, class II, DR alpha - HLA-DRA, major histocompatibility complex, class II, DQ beta 1 - HLADQB1, major histocompatibility complex, class II, DP beta 1 - HLA-DPB1, major histocompatibility complex, class II, DQ alpha 1 - HLA-DQA1, interleukin 11 - IL11, Met proto-oncogene - MET, protocadherin 10 - PCDH10, S100 calcium binding protein B S100B, tumor necrosis factor receptor superfamily, member 19 - TNF19) with efficiency $>85 \%$ were used in SYBR Green based PCR reactions. Each sample was tested in 4 
technical replicates on an ABI Prism 7300 thermal cycler (Applied Biosystems, Foster City, $\mathrm{CA})$. The cycle threshold $(\mathrm{Ct})$ of the housekeeping gene Glyceraldehyde-3-Phosphate Dehydrogenase (GAPDH) was used for normalization of all samples.

\section{miRNome Arrays and $\mathrm{qPCR}$ validation}

Small RNAs were isolated using the $m i r V a n a^{\mathrm{TM}}$ miRNA Isolation Kit (Ambion, Foster City, CA, USA), and cDNA was prepared from it with miScript II RT Kit (Qiagen, Valencia, CA, USA) using miScript HiSpec Buffer. The individual sample cDNAs were pooled in equal proportions into four groups based on gender and age (Supplemental Table S1). The levels of 1008 miRNAs were assessed with Human miRNome miScript miRNA PCR Array (Qiagen, Valencia, CA, USA) according to the manufacturer's instructions and as previously described (26). A $30 \%$ difference between the average $\Delta \mathrm{Ct}$ for MDD and CNTR $(|\Delta \Delta \mathrm{Ct}|$ $>0.3785)$ and a group-wise $p$-value generated by a ttest $(\mathrm{p}<0.05)$ were used to determine differential expression for each miRNA.

Custom generated miScript miRNA PCR Arrays (Qiagen, Valencia, CA, USA) were used to assay the level of seven miRNAs: hsa-miR-21, hsa-miR-377, hsa-miR-193a-3p, hsamiR-542-3p, hsa-miR-22, hsa-miR-103a, hsa-miR-185. This assessment was performed on all individual samples (MDD $n=16$; CNTR $n=16$ ).

\section{RESULTS}

\section{mRNA signature in MDD fibroblasts}

The experimental design for our study is presented in Supplemental Figure S1. In the first part of the study, cultured fibroblast samples from patients with Major Depressive Disorder (MDD) and matched control (CNTR) subjects ( $\mathrm{n}=16$ pairs, 32 individuals) (Supplemental Table S1) were assayed for differential gene expression using GeneChip HT HG-U133 Plus PM 96 Array Plate (Affymetrix Inc, Santa Clara, CA). We identified 162 differentially expressed gene probes (Supplemental Table S2) that reported $>50 \%$ change, and $\mathrm{p}<0.05$ in both pairwise and groupwise assessment. Of the 162 changed gene probes, representing 139 unique known genes, 25 showed increased expression and 114 had decreased levels in MDD, suggesting a predominant loss of function, rather than induction of gene expression in the diseased subjects. A two-way hierarchical clustering (genes $\times$ samples) of the expression levels of these gene probes resulted in separation the majority of MDD samples to a distinct class (Supplemental Figure S2).

Among the individual genes with the most prominent decrease were protocadherin 10 (PCDH10) $(\mathrm{ALR}=-1.73$, ppval $=0.00154$, gpval $=0.00057)$, tenascin $\mathrm{XB}(\mathrm{TNXB})$ (ALR=-1.30, ppval=0.04545, gpval=0.02515), periplakin $(\mathrm{PPL})(\mathrm{ALR}=-1.34$, ppval $=0.00556$, gpval $=0.02714$ ), and hepatocyte growth factor receptor met (MET) (ALR=-1.21, ppval=0.04474, gpval=0.0012), which are involved in cell-cell communication/adhesion. Of these, due to its known effects on brain development and function, MET attracted particular attention and we decided to assess the expression of genes belonging to the MET intracellular cascade defined by Biocarta in a targeted fashion. Overall, from the 37 genes 16 showed a significant difference (Figure 1) including 
phosphoinositide-3-kinase, regulatory subunit 1 (PIK3R1), hepatocyte growth factor (HGF), GRB2-associated binding protein 1(GAB1), son of sevenless homolog 1 (SOS1), Rap guanine nucleotide exchange factor 1 (RAPGEF1), signal transducer and activator of transcription 3 (STAT3) protein tyrosine phosphatase, non-receptor type 11 (PTPN11), p21/ Cdc42/Rac1-activated kinase 1 (PAK1), mitogen-activated protein kinase 1 (MAPK1), v-crk sarcoma virus CT10 oncogene homolog like (CRKL), jun oncogene (JUN), phosphoinositide-3-kinase, catalytic, alpha polypeptide (PTEN), member of RAS oncogene family (RAP1A) mitogen-activated protein kinase kinase 1 (MAP2K1), and v-Ha-ras Harvey rat sarcoma viral oncogene homolog (HRAS). Furthermore, HGF/MET morphogenic signaling synergizes with v-erb-b2 erythroblastic leukemia viral oncogene homolog 2 (ErbB2) to enhance cell motility (39), and our data also revealed a significant downregulation of ErbB2, further supporting the notion for a deficit in HGF/MET-mediated signaling in MDD fibroblasts.

In addition to a targeted MET pathway analysis, we also performed an unbiased pathway enrichment analysis using Gene Set Enrichment Analyses (38). Using this approach, we identified 10 differentially expressed BioCarta gene sets, six of which were upregulated and 4 were downregulated in MDD samples (Table 1). Interestingly, most of these molecular pathways were related to cell-to-cell communication and are known for their role in the adaptive and innate immune system. These pathway analyses argue that the primary gene expression disturbance in the fibroblasts of MDD patients is in the expression of immune response genes, which has been also reported in the postmortem brain tissue of patients with MDD (12).

\section{qPCR validation of mRNA signature}

Fourteen of the differentially expressed mRNA species were selected for further validation by qPCR. The genes for validation were primarily chosen because of their involvement in the immune system response, a process that has been previously implicated in the pathophysiology of the disease. Twelve of fourteen selected transcripts showed similar, significant expression differences between the control and MDD samples by the two methods, with a high degree of correlation between the microarray-reported ALRs and qPCR-obtained $\Delta \Delta$ Cts $(r=0.84)$ (Supplemental Figure S3), but did not show correlation with body mass index in either dataset. qPCR-reported expression differences between the control and MDD samples were as follows: $\underline{\mathrm{CD} 74}: \Delta \Delta \mathrm{Ct}=2.29, \mathrm{p}=0.012$; HLA-DRA: $\Delta \Delta \mathrm{Ct}$ $=1.61, \mathrm{p}=0.017$; HLA-DQB1: $\Delta \Delta \mathrm{Ct}=1.31, \mathrm{p}=0.018 ;$ IL11: $\Delta \Delta \mathrm{Ct}=1.13, \mathrm{p}=0.017$; HLADPA1: $\Delta \Delta \mathrm{Ct}=1.08, \mathrm{p}=0.011$; S100B: $\Delta \Delta \mathrm{Ct}=1.05, \mathrm{p}=0.031$; $\underline{\mathrm{HBEGF}}: \Delta \Delta \mathrm{Ct}=1.00, \mathrm{p}=0.010$; HLA-DPB1: $\Delta \Delta \mathrm{Ct}=0.43, \mathrm{p}=0.156$; HLA-DQA1: $\Delta \Delta \mathrm{Ct}=0.29, \mathrm{p}=0.285$; MET: $\Delta \Delta \mathrm{Ct}=-0.42$, $\mathrm{p}=0.043$; PCDH10: $\Delta \Delta \mathrm{Ct}=-0.91, \mathrm{p}=0.036 ; \underline{\mathrm{TNF} 19}: \Delta \Delta \mathrm{Ct}=-1.11, \mathrm{p}=0.007$ and $\mathrm{GSTT1}$ : $\Delta \Delta \mathrm{Ct}=-3.77, \mathrm{p}=0.007$.

\section{miRNA signature in MDD fibroblasts}

miRNAs are important control elements in the fine-tuning of gene expression, and are capable of regulating extensive transcriptional networks $(26,40)$. Therefore, we further sought to determine if there was a disturbance of miRNA expression profile in MDD fibroblasts. Small RNA species were isolated from each fibroblast culture, cDNA was 
generated, pooled in 4 groups according to gender and age, and then used to probe Human MirNome arrays, containing assays for 1008 known human miRNAs (26). We observed that approximately $50 \%$ (561) of the tested miRNAs were expressed in cultured dermal fibroblasts. We used a dual expression-significance criteria (26) for determining differential miRNA expression ( $>30 \%$ change, $\mathrm{p}<0.05)$. We detected 38 miRNAs with distinctly different expression in MDD fibroblasts compared to healthy controls (Supplemental Table S3). Of these, 17 miRNAs reported lower and 21 higher levels in MDD fibroblasts. Using unsupervised two-way hierarchical clustering of expression levels, these 38 miRNAs provided a clear separation basis of the MDD and CNTR samples (Figure 2).

Since the miRNA levels were assayed on 4 pooled sets of samples, we sought to validate the observed differences in individual samples. For this purpose, we created custom qPCR arrays containing assays for 7 differentially expressed miRNAs; hsa-miR-21*, hsa-miR-377, hsa-miR-193a-3p, hsa-miR-542-3p, hsa-miR-22, hsa-miR-103a, hsa-miR-185. miRNA expression levels for these 7 miRNA species showed an extremely high correlation $\left(\mathrm{R}^{2}=\right.$ $0.93, \mathrm{p}<0.001$ ) between the pooled sample and individual sample assessments, suggesting that the observations made using the combined samples were not an artifact generated by the pooling (Figure 3).

\section{Cross-talk between miRNA and mRNA expression in MDD fibroblasts}

Next, we tested if the miRNA signature can explain some of the mRNA signature in MDD fibroblasts. To achieve this, we identified all predicted target genes/mRNAs for each of the 38 miRNAs using miRDB online database for miRNA target prediction and functional annotations (41),(42) and tested if the targeted mRNAs were enriched in the transcriptome profile of MDD fibroblasts (Table 2). 32 out of 38 miRNAs (89\%) had at least one target mRNA that was differentially expressed between the MDD and control samples. Furthermore, $51 \%$ of the differentially expressed mRNAs were targets of at least one of the 38 miRNAs that were differentially expressed in the MDD fibroblasts. These findings suggest that the miRNA-mRNA expression signatures of MDD fibroblasts are strongly interconnected.

miRNAs are most commonly considered negative regulators of mRNA expression $(44,45)$, so we hypothesized that the miRNA and their mRNA targets will show expression changes in the opposite direction. Indeed, we found that 28 (76\%) of the miRNAs with altered expression had mRNA targets changed in the opposite direction. Acknowledging the fact that different prediction algorithms produce different sets of target genes, we also performed a secondary identification of miRNA targets using TargetScan Release 6.2 (http:// www.targetscan.org/) (43). TargetScan identified miRNA targets (Supplemental Table S4) that were somewhat different from the ones identified by miRDB, but strikingly similar in terms of relating the miRNA and the mRNA changes in MDD; 65\% (TargetScan) and 76\% (MiRDB) of the differentially changed miRNAs had mRNA targets that were changed in the opposite direction. Regardless of these differences, the findings across the two databases suggest a functionally interconnected miRNA-mRNA network disturbance in MDD fibroblasts, where many (if not the majority) of the mRNA changes are miRNA driven. Nevertheless, it remains challenging to establish which specific miRNA species control 
what individual mRNA transcripts, as $64 \%$ of the differentially expressed mRNAs in MDD fibroblasts were hypothetically controlled by more than one miRNA.

\section{DISCUSSION}

Our data revealed that 1) MDD fibroblasts, when compared to matched controls, show a strong mRNA gene expression difference in molecular networks known to engage in cell-tocell communication and innate/adaptive immunity; 2) the same patient fibroblasts showed altered expression in a distinct panel of 38 miRNAs, which appeared to target many of the differentially expressed mRNAs; 3 ) the miRNA-mRNA expression changes appeared to be functionally connected, as the majority of the miRNA and mRNA changes were in the opposite direction.

These findings have conceptual implications for our understanding of MDD pathophysiology. Our findings reinforce the notion that MDD is not only a disease of the brain, but molecular deficiencies in MDD patients are detectable in other peripheral organs $(28,46)$, including dermal fibroblasts. In addition, it is important to note that the observed mRNA and miRNA changes in the patient fibroblasts are most likely driven by genetic susceptibility to the disease, rather than effects of the environment and lifestyle: most of the epigenetic changes, environmental influences and drug effects are likely to disappear over time, as the fibroblasts continuously divide in the culture $(25,26)$. However, our experiments cannot exclude the possibility that some of the extremely stable, cell-divisionresistant epigenetic factors might also contribute to the observed changes $(47,48)$. In summary, the molecular changes observed in cultured fibroblasts of MDD patients can provide us clues about lifestyle- and medication-independent, conserved disturbances in MDD across the various tissue types.

An unexpected and interesting finding of this study is the strikingly different level of expression of approximately half of the Biocarta defined hepatocyte growth factor receptor met (MET) pathway genes. MET is a receptor tyrosine kinase activated by the hepatocyte growth factor (HGF) and affecting cellular signaling pathways involved in control of proliferation, motility, migration and invasion. Although the functions of MET have been primarily studied in the context of cancer (49), MET signaling is also known to be important in brain development (50) and the regulation of immune cells (51). Importantly, in a recent study HGF was the most highly associated plasma analyte with depressive symptoms (52). Furthermore, the met pathway facilitates adult neurogenesis (53), a process that is significantly impaired in major depression (54).

Immune system disturbances in MDD appear to be an integral part of the disease process $(22,55,56)$. The overlap between symptoms of infectious diseases and common affective manifestations (57) suggested a shared pathophysiology between these two systems, which was investigated in a host of follow-up studies. Ultimately, the combined findings lead to the formulation of the inflammatory and neurodegenerative (I\&ND) hypothesis of MDD (46), which states that MDD is a consequence of neurodegeneration and reduced neurogenesis that arise from inflammation and cell-mediated immune activation. Today, this view is supported by converging data obtained across different methods and systems. First, 
postmortem findings identified that MDD patients show a strong dysregulation of immune system pathways in the brain, encompassing altered levels of pro- and anti-inflammatory cytokine expression of IL-1a, IL-2, IL-3, IL-5, IL-8, IL-9, IL-10, IL-12A, IL-13, IL-15, IL-18, IFN- $\gamma$ and lymphotoxin- $\alpha$ (12). Similarly, altered mRNA levels of immune system genes were found in the neocortex of subjects who committed suicide $(58,59)$. Second, the immune deficits appear to be systemic in patients with MDD, and that they are readily detectable in the peripheral tissues (2). Inflammation and immune system biomarkers of MDD might include C-reactive protein (CRP), cytokines (in particular tumor necrosis factor-a (TNF-a) and interleukin-6 (IL-6)), neopterin and tryptophan catabolites (reviewed by (28)). Third, while in the most recent genome-wide association study of MDD no SNPs achieved genome-wide significance in the MDD discovery or replication phase (60), candidate gene studies hint that genetic vulnerability in immune system genes might predispose to MDD in a complex pattern (61-64). However, it is also clear that the immune system genes are only partially responsible for the genetic vulnerability of MDD, as a recent pathway analysis found that genetic elements regulating growth and organ development might also represent vulnerability factors (65). Our results are also aligned with this overall view: the disturbance in classic complement pathway, cell-to-cell communication and innate/adaptive immunity suggest an integrated immune system and growth disturbance in the patient population.

Several miRNAs have been already associated with treatment response or an increased risk for major depression $(18,66-68)$. Recent reports suggest that polymorphism in the miR-30e precursor is associated with major depressive disorder risk (69), and that miRNA expression was significantly and globally down-regulated in prefrontal cortex of depressed suicide subjects (19). In addition, abnormal processing of pre-miR-182 (a circadian clock modulator) was found in major depression patients with insomnia (70). Unfortunately, due to lack of replication, specific experimental design, differences in analyzed tissue and variety of cohorts the data are challenging to interpret and combine into a comprehensive view in a context of MDD. However, it is clear that miRNAs play a pivotal role in a vast variety of MDD-relevant biological processes, including synaptic plasticity, neurogenesis, and stress response (for a review, see (18)). Thus, it is worth pointing out that at least 8 of the 38 differentially expressed miRNAs that we identified in our MDD-fibroblast screen have been previously implicated in either pathophysiology of psychiatric disorders or pathophysiological processes relevant to MDD: 1) miR-32 and miR-22 levels were altered in both our and in a bipolar postmortem dataset (71), 2) miR-22 represses BDNF, serotonin receptor $2 \mathrm{C}$ (HTR2C), monoamine oxidase A (MAO-A), and the regulator of $\mathrm{G}$ protein signaling (RGS2) (72), 3) miR-196 plays an important regulatory role in schizophrenia (73), 4) miR-132 regulates neurite outgrowth and dendritic morphogenesis (74-76), 5) miR-16 acts as a central effector in 5-HT transporter regulation, mediating the adaptive response of serotonergic and noradrenergic neurons to SSRI antidepressant treatment (77), 6) miR-7 expression is altered in the prefrontal cortex of schizophrenia patients (71), 7) miR-429 is downregulated in response to repeated shocks in a rodent model (78), and 8) miR-107 associated with accelerated disease progression in Alzheimer's disease through regulation of BACE1 ( $\beta$-site amyloid precursor protein-cleaving enzyme 1) (79). 
Still, in our study, the most promising miRNA candidate for diagnostic biomarker was hsamiR-122, with a 350\% decrease in MDD cases at $\mathrm{p}<0.00004$. Hsa-miR-122 is highly expressed in the hippocampus (80) and has been implicated in regulation of fatty acid metabolism $(81,82)$ and circadian rhythm (83). Unfortunately, its role in the central nervous system has not been well established, and understanding its function in the brain requires further studies.

In summary, we believe that a combined miRNA-mRNA analysis approach has a potential to uncover reliable, disease-related panels of biomarkers. The mRNA/miRNA panel identified in this study represents a promising lead, but it will have to be further validated on a different cohort of patients and across different experimental manipulations. Even through in our data sets we did not observe an association between expression and body mass index recorded at the time of the material harvest, we cannot exclude the possibility that some of the observed mRNA/miRNA changes might be related to confounding factors such as life style.

We wish to emphasize that dermal fibroblasts are an appealing experimental model. First, they are easy to obtain and propagate. Second, they are not genetically modified and/or immortalized, yet, the resource is not easily depleted. Third, due to their division in the cell culture, many confounds and epigenetic changes disappear over time. Fourth, our current and previous data suggests that the miRNA and mRNA expression profiles of the fibroblasts from MDD patients are likely to be related to the changes that occur in the brain $(25,30,84$, 85). Fifth, this model is potentially well-suited for in vitro drug screening. Finally, establishing this patient-derived model requires only minimal technical and financial resources, and is ideally suited for smaller laboratories. In addition, should the need arise in follow-up experiments, patient fibroblasts can be transformed into IPSCs that can be differentiated into neuronal lineages (86).

\section{Supplementary Material}

Refer to Web version on PubMed Central for supplementary material.

\section{Acknowledgments}

KM's work is supported by NIMH grants R01MH067234 and R01 MH079299. RCS work was supported by NIMH grants MH01741, MH52339, and MH073630, and a grant from the Brain \& Behavior Research Foundation. We are especially grateful to Weining Xu, Senior Manager at Qiagen, for her invaluable guidance in the use of the miRNome arrays, and the Vantage Molecular Biology Core at Vanderbilt for their help in carrying out the microarray and miRNome array studies.

\section{References}

1. Schlaak JF, Trippler M, Hoyo-Becerra C, Erim Y, Kis B, Wang B, et al. Selective hyperresponsiveness of the interferon system in major depressive disorders and depression induced by interferon therapy. PLoS One. 2012; 7:e38668. [PubMed: 22701688]

2. Hepgul N, Cattaneo A, Zunszain PA, Pariante CM. Depression pathogenesis and treatment: what can we learn from blood mRNA expression? BMC Med. 2013; 11:28. [PubMed: 23384232]

3. Myint AM. Kynurenines: from the perspective of major psychiatric disorders. FEBS J. 2012; 279:1375-1385. [PubMed: 22404766] 
4. Chung CP, Schmidt D, Stein CM, Morrow JD, Salomon RM. Increased oxidative stress in patients with depression and its relationship to treatment. Psychiatry Res. 2012; 206:213-216. [PubMed: 23245537]

5. Suri R, Hellemann G, Cohen L, Aquino A, Altshuler L. Saliva estriol levels in women with and without prenatal antidepressant treatment. Biol Psychiatry. 2008; 64:533-537. [PubMed: 18495086]

6. Raedler TJ. Inflammatory mechanisms in major depressive disorder. Curr Opin Psychiatry. 2011; 24:519-525. [PubMed: 21897249]

7. Craddock N, Sklar P. Genetics of bipolar disorder. Lancet. 2013; 381:1654-1662. [PubMed: 23663951]

8. Israel MA, Yuan SH, Bardy C, Reyna SM, Mu Y, Herrera C, et al. Probing sporadic and familial Alzheimer's disease using induced pluripotent stem cells. Nature. 2012; 482:216-220. [PubMed: 22278060]

9. Hrvoj-Mihic B, Marchetto MC, Gage FH, Semendeferi K, Muotri AR. Novel Tools, Classic Techniques: Evolutionary Studies Using Primate Pluripotent Stem Cells. Biol Psychiatry. 2013

10. Shcheglovitov A, Shcheglovitova O, Yazawa M, Portmann T, Shu R, Sebastiano V, et al. SHANK3 and IGF1 restore synaptic deficits in neurons from 22q13 deletion syndrome patients. Nature. 2013

11. Horvath S, Mirnics K. Immune System Disturbances in Schizophrenia. Biol Psychiatry. 2013

12. Shelton RC, Claiborne J, Sidoryk-Wegrzynowicz M, Reddy R, Aschner M, Lewis DA, et al. Altered expression of genes involved in inflammation and apoptosis in frontal cortex in major depression. Mol Psychiatry. 2010; 16:751-762. [PubMed: 20479761]

13. Sibille E, Wang Y, Joeyen-Waldorf J, Gaiteri C, Surget A, Oh S, et al. A molecular signature of depression in the amygdala. Am J Psychiatry. 2009; 166:1011-1024. [PubMed: 19605536]

14. Tochigi M, Iwamoto K, Bundo M, Sasaki T, Kato N, Kato T. Gene expression profiling of major depression and suicide in the prefrontal cortex of postmortem brains. Neurosci Res. 2008; 60:184191. [PubMed: 18068248]

15. Kang HJ, Adams DH, Simen A, Simen BB, Rajkowska G, Stockmeier CA, et al. Gene expression profiling in postmortem prefrontal cortex of major depressive disorder. J Neurosci. 2007; 27:13329-13340. [PubMed: 18045927]

16. Evans SJ, Choudary PV, Neal CR, Li JZ, Vawter MP, Tomita H, et al. Dysregulation of the fibroblast growth factor system in major depression. Proc Natl Acad Sci U S A. 2004; 101:1550615511. [PubMed: 15483108]

17. Edgar N, Sibille E. A putative functional role for oligodendrocytes in mood regulation. Transl Psychiatry. 2012; 2:e109. [PubMed: 22832953]

18. Dwivedi Y. Evidence demonstrating role of microRNAs in the etiopathology of major depression. J Chem Neuroanat. 2011; 42:142-156. [PubMed: 21515361]

19. Smalheiser NR, Lugli G, Rizavi HS, Torvik VI, Turecki G, Dwivedi Y. MicroRNA expression is down-regulated and reorganized in prefrontal cortex of depressed suicide subjects. PLoS One. 2012; 7:e33201. [PubMed: 22427989]

20. Schmidt HD, Shelton RC, Duman RS. Functional biomarkers of depression: diagnosis, treatment, and pathophysiology. Neuropsychopharmacology. 2011; 36:2375-2394. [PubMed: 21814182]

21. Nemeroff CB, Goldschmidt-Clermont PJ. Heartache and heartbreak--the link between depression and cardiovascular disease. Nat Rev Cardiol. 2012; 9:526-539. [PubMed: 22733213]

22. Miller AH, Maletic V, Raison CL. Inflammation and its discontents: the role of cytokines in the pathophysiology of major depression. Biol Psychiatry. 2009; 65:732-741. [PubMed: 19150053]

23. Belzeaux R, Bergon A, Jeanjean V, Loriod B, Formisano-Treziny C, Verrier L, et al. Responder and nonresponder patients exhibit different peripheral transcriptional signatures during major depressive episode. Transl Psychiatry. 2012; 2:e185. [PubMed: 23149449]

24. Manier DH, Shelton RC, Ellis TC, Peterson CS, Eiring A, Sulser F. Human fibroblasts as a relevant model to study signal transduction in affective disorders. J Affect Disord. 2000; 61:51-58. [PubMed: 11099740]

25. Gibson SA, Korade Z, Shelton RC. Oxidative stress and glutathione response in tissue cultures from persons with major depression. J Psychiatr Res. 2012; 46:1326-1332. [PubMed: 22841833] 
26. Kalman S, Garbett KA, Vereczkei A, Shelton RC, Korade Z, Mirnics K. Metabolic stress-induced microRNA and mRNA expression profiles of human fibroblasts. Exp Cell Res. 2013 in press.

27. Scapagnini G, Davinelli S, Drago F, De Lorenzo A, Oriani G. Antioxidants as antidepressants: fact or fiction? CNS Drugs. 2012; 26:477-490. [PubMed: 22668245]

28. Lopresti AL, Maker GL, Hood SD, Drummond PD. A review of peripheral biomarkers in major depression: The potential of inflammatory and oxidative stress biomarkers. Prog Neuropsychopharmacol Biol Psychiatry. 2013; 48C:102-111. [PubMed: 24104186]

29. First MB, Pincus HA. The DSM-IV Text Revision: rationale and potential impact on clinical practice. Psychiatr Serv. 2002; 53:288-292. [PubMed: 11875221]

30. Akin D, Manier DH, Sanders-Bush E, Shelton RC. Decreased serotonin 5-HT2A receptorstimulated phosphoinositide signaling in fibroblasts from melancholic depressed patients. Neuropsychopharmacology. 2004; 29:2081-2087. [PubMed: 15187984]

31. Irizarry RA, Hobbs B, Collin F, Beazer-Barclay YD, Antonellis KJ, Scherf U, et al. Exploration, normalization, and summaries of high density oligonucleotide array probe level data. Biostatistics. 2003; 4:249-264. [PubMed: 12925520]

32. Lazarov O, Robinson J, Tang YP, Hairston IS, Korade-Mirnics Z, Lee VM, et al. Environmental enrichment reduces Abeta levels and amyloid deposition in transgenic mice. Cell. 2005; 120:701713. [PubMed: 15766532]

33. Unger T, Korade Z, Lazarov O, Terrano D, Schor NF, Sisodia SS, et al. Transcriptome differences between the frontal cortex and hippocampus of wild-type and humanized presenilin-1 transgenic mice. Am J Geriatr Psychiatry. 2005; 13:1041-1051. [PubMed: 16319296]

34. Unger T, Korade Z, Lazarov O, Terrano D, Sisodia SS, Mirnics K. True and false discovery in DNA microarray experiments: transcriptome changes in the hippocampus of presenilin 1 mutant mice. Methods. 2005; 37:261-273. [PubMed: 16308155]

35. Arion D, Unger T, Lewis DA, Levitt P, Mirnics K. Molecular evidence for increased expression of genes related to immune and chaperone function in the prefrontal cortex in schizophrenia. Biol Psychiatry. 2007; 62:711-721. [PubMed: 17568569]

36. Glorioso C, Sabatini M, Unger T, Hashimoto T, Monteggia LM, Lewis DA, et al. Specificity and timing of neocortical transcriptome changes in response to BDNF gene ablation during embryogenesis or adulthood. Mol Psychiatry. 2006; 11:633-648. [PubMed: 16702976]

37. Kuehn H, Liberzon A, Reich M, Mesirov JP. Using GenePattern for gene expression analysis. Curr Protoc Bioinformatics. 2008; Chapter 7:Unit 7-12.

38. Subramanian A, Kuehn H, Gould J, Tamayo P, Mesirov JP. GSEA-P: a desktop application for Gene Set Enrichment Analysis. Bioinformatics. 2007; 23:3251-3253. [PubMed: 17644558]

39. Khoury H, Naujokas MA, Zuo D, Sangwan V, Frigault MM, Petkiewicz S, et al. HGF converts ErbB2/Neu epithelial morphogenesis to cell invasion. Mol Biol Cell. 2005; 16:550-561. [PubMed: $15548598]$

40. O'Carroll D, Schaefer A. General principals of miRNA biogenesis and regulation in the brain. Neuropsychopharmacology. 2012; 38:39-54. [PubMed: 22669168]

41. Wang X, El Naqa IM. Prediction of both conserved and nonconserved microRNA targets in animals. Bioinformatics. 2008; 24:325-332. [PubMed: 18048393]

42. Wang X. miRDB: a microRNA target prediction and functional annotation database with a wiki interface. RNA. 2008; 14:1012-1017. [PubMed: 18426918]

43. Friedman RC, Farh KK, Burge CB, Bartel DP. Most mammalian mRNAs are conserved targets of microRNAs. Genome Res. 2009; 19:92-105. [PubMed: 18955434]

44. Carrington JC, Ambros V. Role of microRNAs in plant and animal development. Science. 2003; 301:336-338. [PubMed: 12869753]

45. Zhang N, Lin JK, Chen J, Liu XF, Liu JL, Luo HS, et al. MicroRNA 375 mediates the signaling pathway of corticotropin-releasing factor (CRF) regulating pro-opiomelanocortin (POMC) expression by targeting mitogen-activated protein kinase 8. J Biol Chem. 2013; 288:10361-10373. [PubMed: 23430746]

46. Maes M, Yirmyia R, Noraberg J, Brene S, Hibbeln J, Perini G, et al. The inflammatory \& neurodegenerative (I\&ND) hypothesis of depression: leads for future research and new drug developments in depression. Metab Brain Dis. 2009; 24:27-53. [PubMed: 19085093] 
47. Hoffmann A, Spengler D. DNA memories of early social life. Neuroscience. 2012

48. Stringer JM, Barrand S, Western P. Fine-tuning evolution: germ-line epigenetics and inheritance. Reproduction. 2013; 146:R37-48. [PubMed: 23633622]

49. Organ SL, Tsao MS. An overview of the c-MET signaling pathway. Ther Adv Med Oncol. 2011; 3:S7-S19. [PubMed: 22128289]

50. Maina F, Klein R. Hepatocyte growth factor, a versatile signal for developing neurons. Nat Neurosci. 1999; 2:213-217. [PubMed: 10195212]

51. van der Voort R, Taher TE, Derksen PW, Spaargaren M, van der Neut R, Pals ST. The hepatocyte growth factor/Met pathway in development, tumorigenesis, and B-cell differentiation. Adv Cancer Res. 2000; 79:39-90. [PubMed: 10818677]

52. Arnold SE, Xie SX, Leung YY, Wang LS, Kling MA, Han X, et al. Plasma biomarkers of depressive symptoms in older adults. Transl Psychiatry. 2012; 2:e65. [PubMed: 22832727]

53. Nakaguchi K, Jinnou H, Kaneko N, Sawada M, Hikita T, Saitoh S, et al. Growth factors released from gelatin hydrogel microspheres increase new neurons in the adult mouse brain. Stem Cells Int. 2012; 2012:915160. [PubMed: 23093979]

54. Tanti A, Belzung C. Neurogenesis along the septo-temporal axis of the hippocampus: are depression and the action of antidepressants region-specific? Neuroscience. 2013; 252:234-252. [PubMed: 23973415]

55. Raison CL, Capuron L, Miller AH. Cytokines sing the blues: inflammation and the pathogenesis of depression. Trends Immunol. 2006; 27:24-31. [PubMed: 16316783]

56. Raison CL, Miller AH. Is depression an inflammatory disorder? Curr Psychiatry Rep. 2011; 13:467-475. [PubMed: 21927805]

57. Licinio J, Frost P. The neuroimmune-endocrine axis: pathophysiological implications for the central nervous system cytokines and hypothalamus-pituitary-adrenal hormone dynamics. Braz $\mathrm{J}$ Med Biol Res. 2000; 33:1141-1148. [PubMed: 11004714]

58. Pandey GN, Rizavi HS, Ren X, Fareed J, Hoppensteadt DA, Roberts RC, et al. Proinflammatory cytokines in the prefrontal cortex of teenage suicide victims. J Psychiatr Res. 2011; 46:57-63. [PubMed: 21906753]

59. Galfalvy H, Zalsman G, Huang YY, Murphy L, Rosoklija G, Dwork AJ, et al. A pilot genome wide association and gene expression array study of suicide with and without major depression. World J Biol Psychiatry. 2011

60. Ripke S, Wray NR, Lewis CM, Hamilton SP, Weissman MM, Breen G, et al. A mega-analysis of genome-wide association studies for major depressive disorder. Mol Psychiatry. 2012; 18:497511. [PubMed: 22472876]

61. Song GG, Kim JH, Lee YH. Genome-wide pathway analysis in major depressive disorder. J Mol Neurosci. 2013; 51:428-436. [PubMed: 23794217]

62. Jia P, Kao CF, Kuo PH, Zhao Z. A comprehensive network and pathway analysis of candidate genes in major depressive disorder. BMC Syst Biol. 2012; 5(Suppl 3):S12. [PubMed: 22784618]

63. Su S, Miller AH, Snieder H, Bremner JD, Ritchie J, Maisano C, et al. Common genetic contributions to depressive symptoms and inflammatory markers in middle-aged men: the Twins Heart Study. Psychosom Med. 2009; 71:152-158. [PubMed: 19073752]

64. Wong ML, Dong C, Maestre-Mesa J, Licinio J. Polymorphisms in inflammation-related genes are associated with susceptibility to major depression and antidepressant response. Mol Psychiatry. 2008; 13:800-812. [PubMed: 18504423]

65. Wong ML, Dong C, Andreev V, Arcos-Burgos M, Licinio J. Prediction of susceptibility to major depression by a model of interactions of multiple functional genetic variants and environmental factors. Mol Psychiatry. 2012; 17:624-633. [PubMed: 22449891]

66. Serafini G, Pompili M, Hansen KF, Obrietan K, Dwivedi Y, Shomron N, et al. The Involvement of MicroRNAs in Major Depression, Suicidal Behavior, and Related Disorders: A Focus on miR-185 and miR-491-3p. Cell Mol Neurobiol. 2013

67. Oved K, Morag A, Pasmanik-Chor M, Oron-Karni V, Shomron N, Rehavi M, et al. Genome-wide miRNA expression profiling of human lymphoblastoid cell lines identifies tentative SSRI antidepressant response biomarkers. Pharmacogenomics. 2012; 13:1129-1139. [PubMed: 22909203] 
68. Mouillet-Richard S, Baudry A, Launay JM, Kellermann O. MicroRNAs and depression. Neurobiol Dis. 2012; 46:272-278. [PubMed: 22226785]

69. Xu Y, Liu H, Li F, Sun N, Ren Y, Liu Z, et al. A polymorphism in the microRNA-30e precursor associated with major depressive disorder risk and P300 waveform. J Affect Disord. 2010; 127:332-336. [PubMed: 20579744]

70. Saus E, Soria V, Escaramis G, Vivarelli F, Crespo JM, Kagerbauer B, et al. Genetic variants and abnormal processing of pre-miR-182, a circadian clock modulator, in major depression patients with late insomnia. Hum Mol Genet. 2010; 19:4017-4025. [PubMed: 20656788]

71. Kim AH, Reimers M, Maher B, Williamson V, McMichael O, McClay JL, et al. MicroRNA expression profiling in the prefrontal cortex of individuals affected with schizophrenia and bipolar disorders. Schizophr Res. 2010; 124:183-191. [PubMed: 20675101]

72. Muinos-Gimeno M, Espinosa-Parrilla Y, Guidi M, Kagerbauer B, Sipila T, Maron E, et al. Human microRNAs miR-22, miR-138-2, miR-148a, and miR-488 are associated with panic disorder and regulate several anxiety candidate genes and related pathways. Biol Psychiatry. 2011; 69:526-533. [PubMed: 21168126]

73. Guo AY, Sun J, Jia P, Zhao Z. A novel microRNA and transcription factor mediated regulatory network in schizophrenia. BMC Syst Biol. 2010; 4:10. [PubMed: 20156358]

74. Kawashima H, Numakawa T, Kumamaru E, Adachi N, Mizuno H, Ninomiya M, et al. Glucocorticoid attenuates brain-derived neurotrophic factor-dependent upregulation of glutamate receptors via the suppression of microRNA-132 expression. Neuroscience. 2010; 165:1301-1311. [PubMed: 19958814]

75. Vo N, Klein ME, Varlamova O, Keller DM, Yamamoto T, Goodman RH, et al. A cAMP-response element binding protein-induced microRNA regulates neuronal morphogenesis. Proc Natl Acad Sci U S A. 2005; 102:16426-16431. [PubMed: 16260724]

76. Wayman GA, Davare M, Ando H, Fortin D, Varlamova O, Cheng HY, et al. An activity-regulated microRNA controls dendritic plasticity by down-regulating p250GAP. Proc Natl Acad Sci U S A. 2008; 105:9093-9098. [PubMed: 18577589]

77. Baudry A, Mouillet-Richard S, Schneider B, Launay JM, Kellermann O. miR-16 targets the serotonin transporter: a new facet for adaptive responses to antidepressants. Science. 2010; 329:1537-1541. [PubMed: 20847275]

78. Smalheiser NR, Lugli G, Rizavi HS, Zhang H, Torvik VI, Pandey GN, et al. MicroRNA expression in rat brain exposed to repeated inescapable shock: differential alterations in learned helplessness vs. non-learned helplessness. Int J Neuropsychopharmacol. 2011; 14:1315-1325. [PubMed: 21275079]

79. Wang WX, Rajeev BW, Stromberg AJ, Ren N, Tang G, Huang Q, et al. The expression of microRNA miR-107 decreases early in Alzheimer's disease and may accelerate disease progression through regulation of beta-site amyloid precursor protein-cleaving enzyme 1 . $\mathbf{J}$ Neurosci. 2008; 28:1213-1223. [PubMed: 18234899]

80. Deng X, Zhong Y, Gu L, Shen W, Guo J. MiR-21 involve in ERK-mediated upregulation of MMP9 in the rat hippocampus following cerebral ischemia. Brain Res Bull. 2013; 94:56-62. [PubMed: 23473787]

81. Lagos-Quintana M, Rauhut R, Yalcin A, Meyer J, Lendeckel W, Tuschl T. Identification of tissuespecific microRNAs from mouse. Curr Biol. 2002; 12:735-739. [PubMed: 12007417]

82. Lynn FC. Meta-regulation: microRNA regulation of glucose and lipid metabolism. Trends Endocrinol Metab. 2009; 20:452-459. [PubMed: 19800254]

83. Gatfield D, Le Martelot G, Vejnar CE, Gerlach D, Schaad O, Fleury-Olela F, et al. Integration of microRNA miR-122 in hepatic circadian gene expression. Genes Dev. 2009; 23:1313-1326. [PubMed: 19487572]

84. Akin D, Manier DH, Sanders-Bush E, Shelton RC. Signal transduction abnormalities in melancholic depression. Int J Neuropsychopharmacol. 2005; 8:5-16. [PubMed: 15500705]

85. Shelton RC, Mainer DH, Sulser F. cAMP-dependent protein kinase activity in major depression. Am J Psychiatry. 1996; 153:1037-1042. [PubMed: 8678172]

86. Fink KD, Crane AT, Leveque X, Dues DJ, Huffman LD, Moore AC, et al. Intrastriatal Transplantation of Adenovirus-Generated Induced Pluripotent Stem Cells for Treating 
Neuropathological and Functional Deficits in a Rodent Model of Huntington's Disease. Stem Cells Transl Med. 2014 


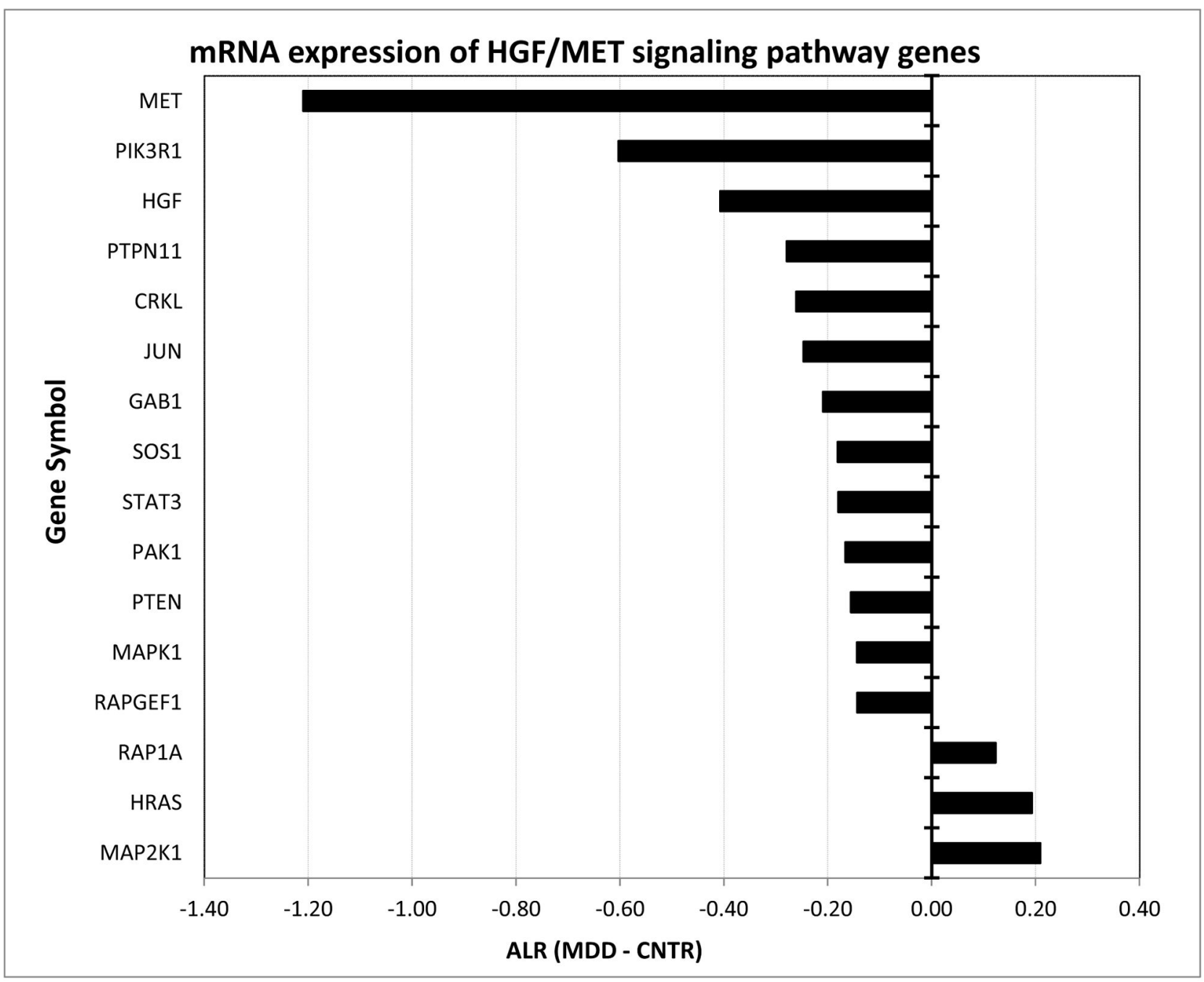

Figure 1. mRNA expression of HGF/MET signaling pathway genes is different between the fibroblasts originating from MDD and CNTRL subjects

$\mathrm{Y}$-axis depicts differentially expressed HGF/MET pathway transcripts as defined by BioCarta/GSEA. X-axis denotes Average Log2 Ratio (ALR) between the MDD and CNTR samples. Bars denote magnitude of change. Note that the vast majority of the genes within this pathway were underexpressed in the MDD fibroblasts. 


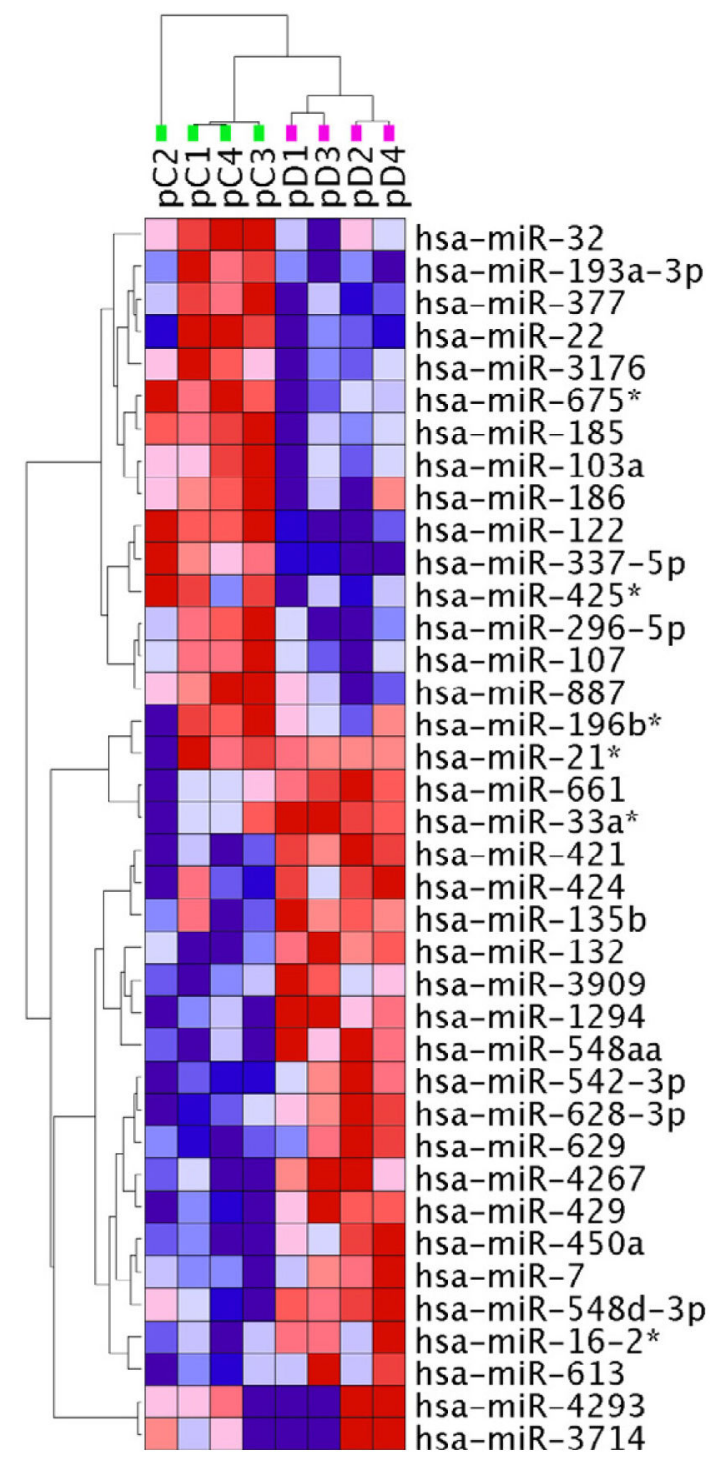

Figure 2. Clustering of miRNAs that are differentially expressed between the MDD and CNTR samples

miRNA levels were assessed in 8 pooled samples (4 CNTR + 4 MDD) using miRNome PCR Arrays. 38 miRNAs reported differential expression between fibroblasts from MDD patients and healthy controls. Unsupervised two-way hierarchical clustering of these miRNA expression levels provided a clear separation between the MDD and CNTR samples.

Samples were clustered vertically, miRNAs were clustered horizontally. Each colored square represents a normalized miRNA expression level, color coded for increase (red) or decrease (blue) from the mean. Color intensity is proportional to magnitude of change. The clustering resulted in a separation of the samples into two groups, perfectly matching the two distinct diagnostic categories (vertical dendrogram: green - CNTR samples, purple MDD samples). For more detail, see Supplemental Figure S2. 


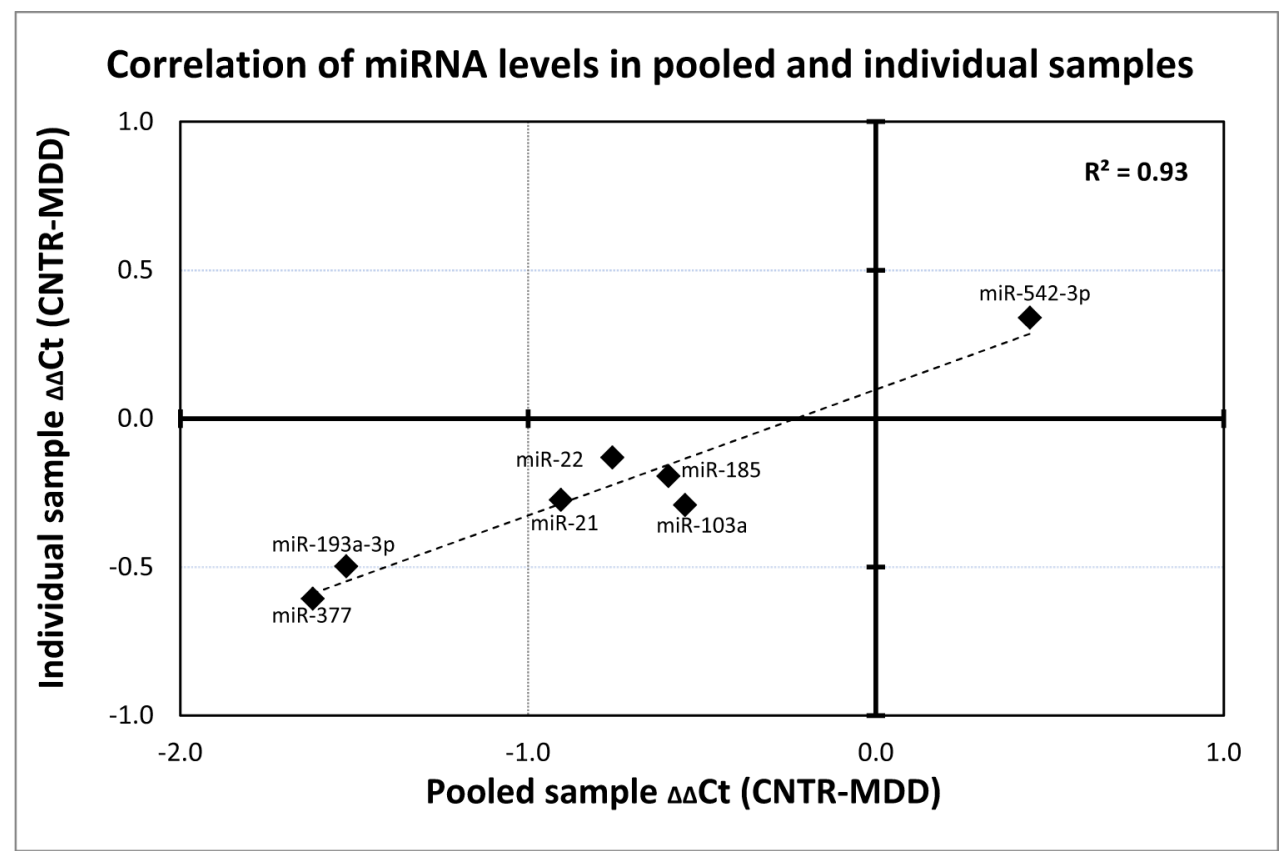

Figure 3. Differential miRNA expression in pooled MDD fibroblast samples validated on individual samples

The expression levels of 7 miRNAs in pooled samples determined by miRNome arrays were validated on individual samples using custom qPCR arrays ( $\mathrm{n}=32,16 \mathrm{MDD}+16 \mathrm{CNTR})$. $\Delta \Delta \mathrm{Ct}$ from the pooled samples are plotted on the $\mathrm{X}$-axis, $\Delta \Delta \mathrm{Cts}$ from individual samples are denoted on the $y$-axis. Individual data points indicate the expression of 7 miRNA species. Note that the two datasets were highly correlated $\left(\mathrm{R}^{2}=0.93, \mathrm{p}<0.001\right)$. 


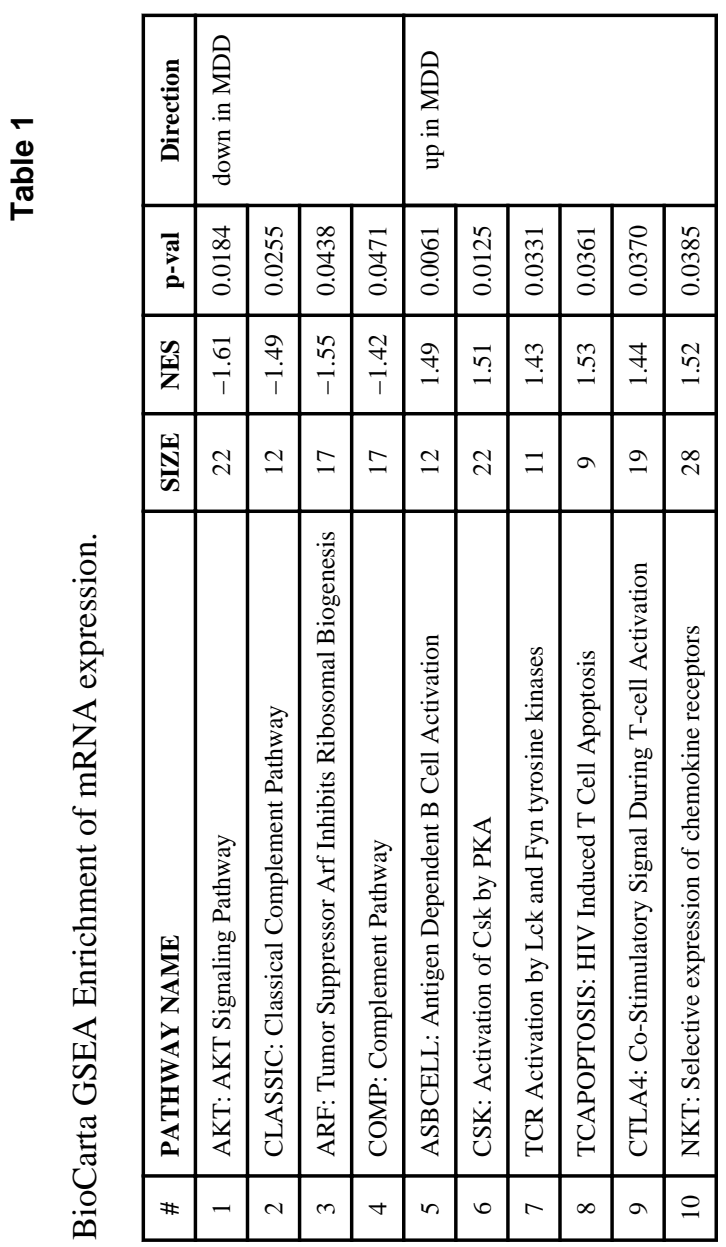

Biol Psychiatry. Author manuscript; available in PMC 2016 February 01. 

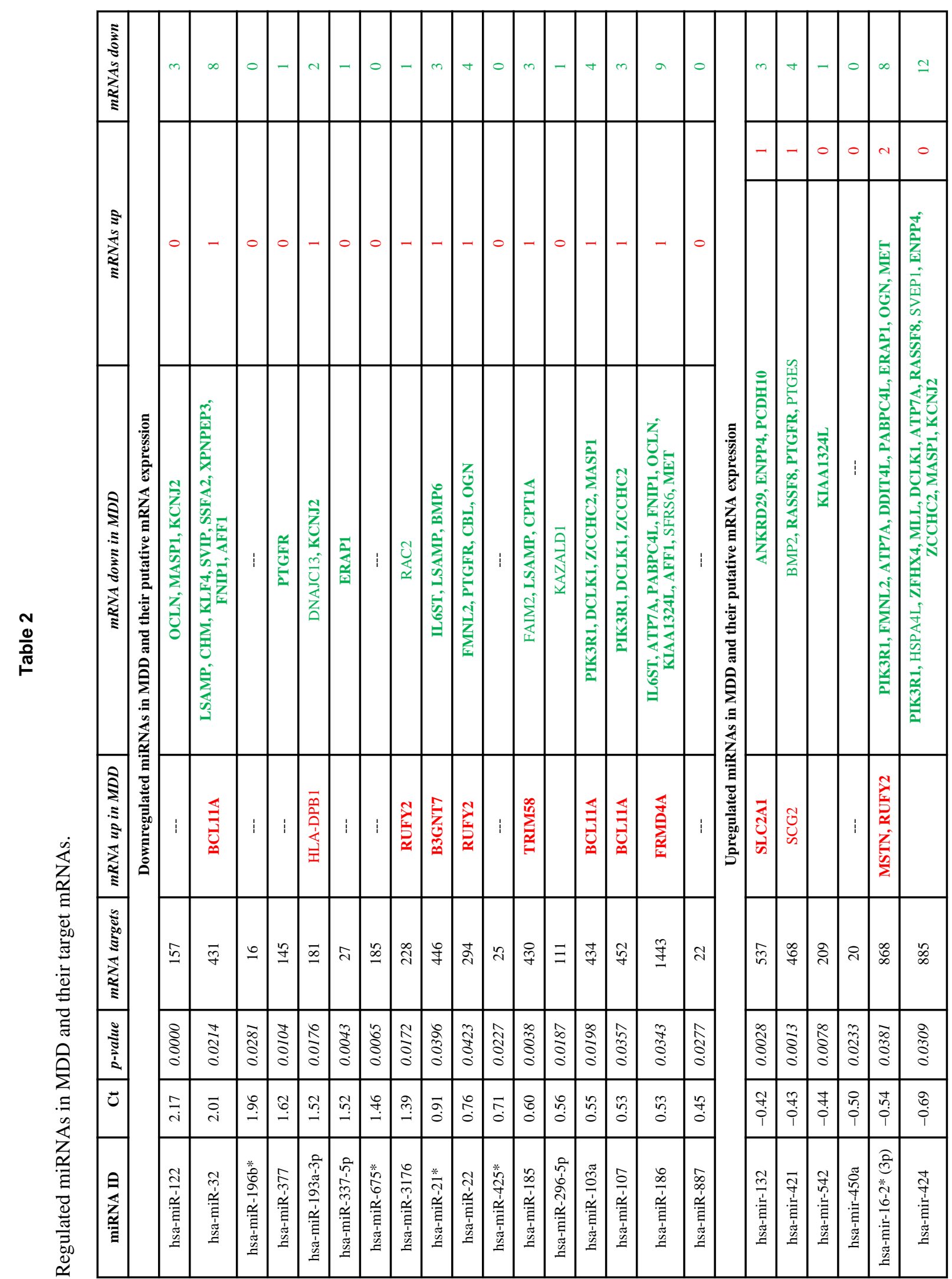

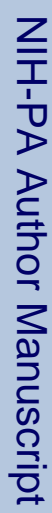

Biol Psychiatry. Author manuscript; available in PMC 2016 February 01. 


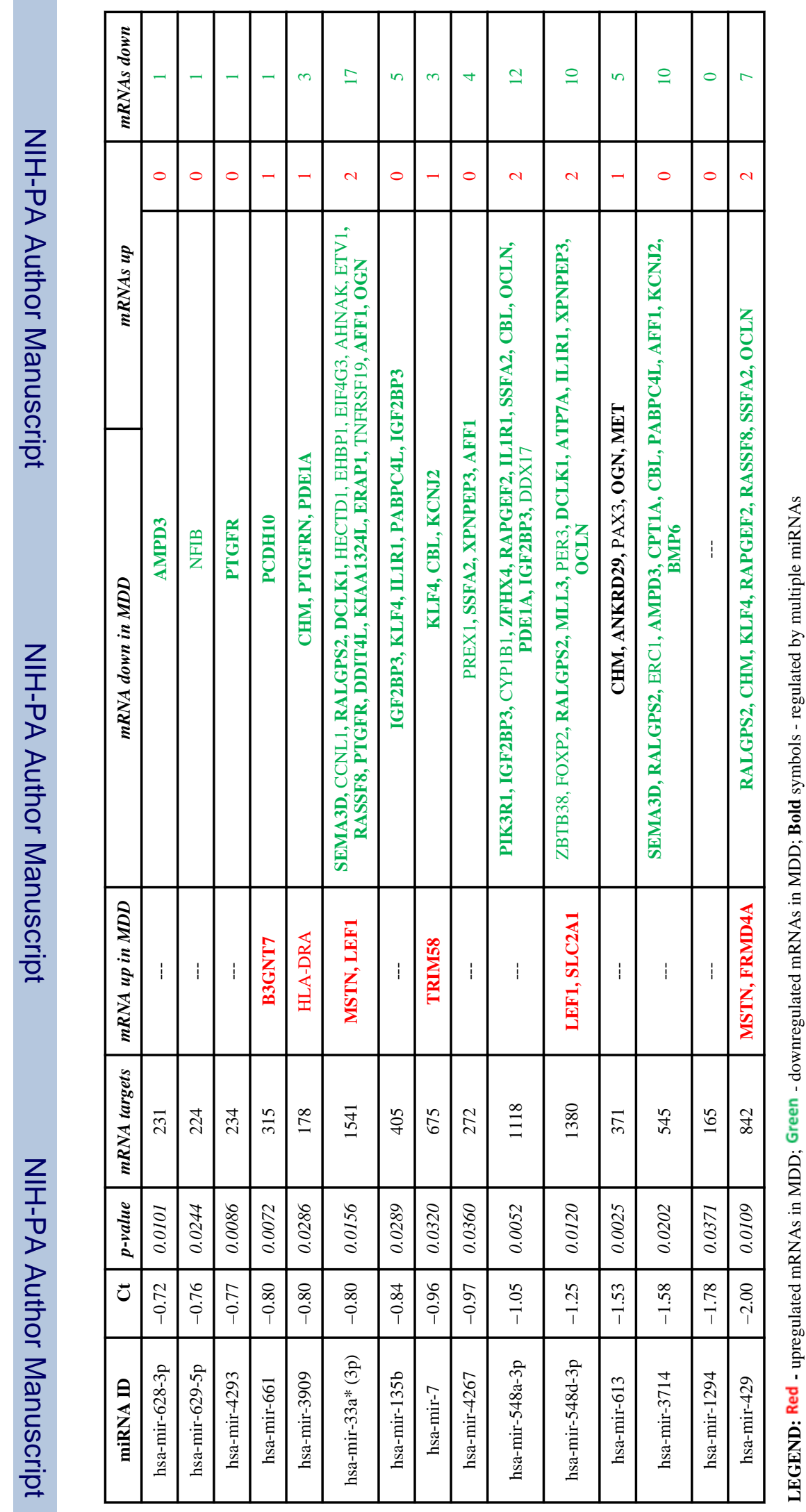




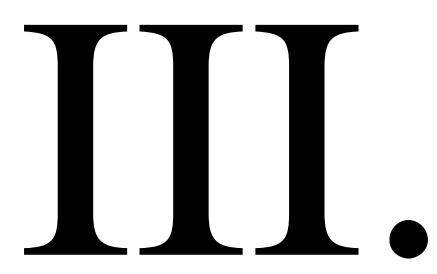




\title{
ORIGINAL ARTICLE
}

\section{Fibroblasts from patients with major depressive disorder show distinct transcriptional response to metabolic stressors}

\author{
KA Garbett ${ }^{1}$, A Vereczkei ${ }^{1,2}$, S Kálmán ${ }^{1,3}$, L Wang $^{4}$, Ž Korade ${ }^{1,5}$, RC Shelton ${ }^{6}$ and K Mirnics ${ }^{1,3,5}$
}

\begin{abstract}
Major depressive disorder (MDD) is increasingly viewed as interplay of environmental stressors and genetic predisposition, and recent data suggest that the disease affects not only the brain, but the entire body. As a result, we aimed at determining whether patients with major depression have aberrant molecular responses to stress in peripheral tissues. We examined the effects of two metabolic stressors, galactose (GAL) or reduced lipids (RL), on the transcriptome and miRNome of human fibroblasts from 16 pairs of patients with MDD and matched healthy controls (CNTR). Our results demonstrate that both MDD and CNTR fibroblasts had a robust molecular response to GAL and RL challenges. Most importantly, a significant part (messenger RNAs (mRNAs): 26-33\%; microRNAs (miRNAs): 81-90\%) of the molecular response was only observed in MDD, but not in CNTR fibroblasts. The applied metabolic challenges uncovered mRNA and miRNA signatures, identifying responses to each stressor characteristic for the MDD fibroblasts. The distinct responses of MDD fibroblasts to GAL and RL revealed an aberrant engagement of molecular pathways, such as apoptosis, regulation of cell cycle, cell migration, metabolic control and energy production. In conclusion, the metabolic challenges evoked by GAL or RL in dermal fibroblasts exposed adaptive dysfunctions on mRNA and miRNA levels that are characteristic for MDD. This finding underscores the need to challenge biological systems to bring out disease-specific deficits, which otherwise might remain hidden under resting conditions.
\end{abstract}

Translational Psychiatry (2015) 5, e523; doi:10.1038/tp.2015.14; published online 10 March 2015

\section{INTRODUCTION}

Major depressive disorder (MDD) is a mental disorder characterized by persistent depressed mood, anhedonia, sleep and appetite disturbances, and feelings of worthlessness, guilt and hopelessness. It is increasingly viewed as an illness of the body as well as of the mind. ${ }^{1}$ Both genetic and environmental factors have been associated with the etiology of the disease, ${ }^{2}$ but their interplay remains unexplained to date. Stressful life events are associated with the onset and severity of major depression; ${ }^{3}$ therefore, we speculated that aberrant adaptive responses to stressors would be also detectable at the cellular level.

Coordination of cellular responses to stress is largely accomplished by mitochondria. ${ }^{4}$ Regardless of the type of stress a cell is experiencing (for example, exposure to harmful chemicals, changes of nutrient or oxygen supplies, dangerous levels of reactive oxygen species or even pathogen invasion), mitochondria utilize a wide array of mechanisms aimed at restoring cellular homeostasis. ${ }^{5}$ Therefore, the health of these cellular organelles is critical not only for the fate of the individual cells, but for the wellbeing of the entire organism. Not surprisingly, abnormalities in mitochondrial functions have been implicated in neurological ${ }^{6}$ and psychiatric disorders, ${ }^{7-9}$ and specifically in MDD. ${ }^{10}$ In addition, mitochondria are involved in multiple metabolic pathways and are the most prominent generators of energy in the cell. Energy is produced in the cytoplasm through glycolysis, or in the mitochondria through oxidative phosphorylation, also known as mitochondrial respiration. The reliance on energy produced by mitochondrial respiration, rather than glycolysis, can be experimentally shifted by changing the sugar source in the cell culture medium from glucose to galactose (GAL). ${ }^{11}$ The shift to oxidative phosphorylation can reveal mitochondrial deficits intersecting control of carbohydrate metabolism and energy homeostasis. Energy production is also tightly interconnected with lipid metabolism through the key metabolite acetyl-CoA. AcetylCoA may remain in the mitochondria for energy production or may be exported for de novo fatty acid synthesis into the cytoplasm, depending mainly on the availability of fatty acids. This adaptive shift can be experimentally directed by limiting the fatty acid supply in the cell culture medium. Therefore, cellular adaptation to stress can be tested by simple manipulations of the carbohydrate and fatty acids source in the culture medium.

Molecular adaptation to stress in the context of mental disorders is difficult to address in the human brain. Alternatively, we used in vitro propagated peripheral cells to test the effects of metabolic stress. Dermal fibroblasts are an appealing cell-based model for studying peripheral events associated with brain disorders, based on the ease of establishing them from skin biopsies, and the ability to maintain in culture without additional reprograming. ${ }^{12}$ In addition, the confounding variability in human samples on the basis of patients' hormones, life style or medication use, are virtually eliminated after several cell divisions. To date, skin fibroblast cultures have been used successfully to elucidate molecular mechanisms associated with schizophrenia $^{13,14}$ and developmental disorders, ${ }^{15,16}$ and for discerning abnormalities in signal transduction pathways in MDD. ${ }^{12,17-19}$ In addition, previous reports for dysregulations in

\footnotetext{
${ }^{1}$ Department of Psychiatry, Vanderbilt University, Nashville, TN, USA; ${ }^{2}$ Department of Medical Chemistry, Molecular Biology and Pathobiochemistry, Semmelweis University, Budapest, Hungary; ${ }^{3}$ Department of Psychiatry, University of Szeged, Szeged, Hungary; ${ }^{4}$ Department of Biostatistics, Vanderbilt University, Nashville, TN, USA; ${ }^{5}$ Vanderbilt Kennedy Center for Research on Human Development, Vanderbilt University, Nashville, TN, USA and ${ }^{6}$ Department of Psychiatry, University of Alabama, Birmingham, AL, USA. Correspondence: Dr KA Garbett, Department of Psychiatry, Vanderbilt University, 8128 MRB III, 465 21st Avenue South, Nashville, TN 37232 , USA.

E-mail: krassimira.garbett@vanderbilt.edu
}

Received 2 October 2014; revised 12 December 2014; accepted 19 December 2014 
lipid metabolism ${ }^{20}$ and mitochondrial respiration, ${ }^{21}$ suggest abnormalities in mitochondrial function in the periphery of MDD patients. Therefore, we aimed at elucidating whether MDD fibroblasts would in addition exhibit molecular deficits in response to metabolic stressors such as GAL and reduced lipids (RL) in the culture medium. In this manuscript, we present the experimental data gathered from metabolically challenged human dermal fibroblasts and discuss their relevance to MDD.

\section{MATERIALS AND METHODS}

\section{Human fibroblasts}

This study was approved by the Vanderbilt University Institutional Review Board. Participants were diagnosed with a current major depressive episode according to the Structured Clinical Interview for DSM-IV-TR ${ }^{22}$ with an exclusion criteria of other primary axis I DSM-IV diagnosis. Procedures for recruitment and diagnosis have been described previously. ${ }^{12,18} \mathrm{~A}$ written informed consent was obtained from all the participants before any procedures were conducted. Sixteen healthy controls (CNTR) were matched by age, race and gender to MDD patients (Supplementary Material 1). The MDD and CNTR subject groups had similar average age $(M D D=34.9, C N T R=35.2)$, representation of gender (12 female and four male) and race (12 white and four African American). Fibroblast cultures were established from skin biopsies according to a protocol previously described. ${ }^{12,18}$

Briefly, fibroblast cultures underwent $5-10$ passages with DMEM (Dulbecco's Modified Eagle's medium; Mediatech, Manassas, VA, USA) containing $250 \mathrm{~mm}$ glucose and $1 \mathrm{mM}$ sodium pyruvate, supplemented with $2 \mathrm{~mm}$ L-glutamine (Mediatech), 10\% fetal bovine serum (Thermo Scientific HyClone, Logan, UT, USA) and antibiotic/antimycotic solution (Invitrogen, Carlsbad, CA, USA). Cultures were maintained at $37^{\circ} \mathrm{C}$ and $5 \%$ $\mathrm{CO}_{2}$. A cell line from each patient was divided into three plates (plating density $1.2 \times 10^{6}$ cells per plate) and after overnight adherence, each plate received one of the following formulations: (1) standard (STD) medium: DMEM containing $25 \mathrm{~mm}$ glucose; (2) galactose-containing (GAL) medium: DMEM with no glucose, supplemented with $10 \mathrm{~mm}$ GAL (Sigma-Aldrich, St. Louis, MO, USA); (3) RL medium: DMEM containing $25 \mathrm{~mm}$ glucose, supplemented with lipid-reduced fetal bovine serum (Thermo Scientific HyClone). All the cultures were grown for 7 days, refreshing the media every 2 days. The cells were collected after washing with ice-cold phosphate-buffered saline twice, and then trypsinized, pelleted $(700 \mathrm{~g}$ for $8 \mathrm{~min}$ at $18^{\circ} \mathrm{C}$ ) and stored at $-80^{\circ} \mathrm{C}$ until RNA isolation. In all the tested cell lines, neither GAL nor RL exhibited a detectable effect on cell growth and mortality.

\section{RNA isolation and analyses}

Total RNA and small RNA fractions were isolated from frozen fibroblasts using mirVana microRNA (miRNA) isolation Kit (Ambion, Austin, TX, USA) according to manufacturer's instruction. Both total RNA and small RNA were eluted with $100 \mu \mathrm{l}$ Elution Solution. Agilent 2100 Bioanalyzer was used to determine the quality and size of the RNA preps. Total RNA integrity number for all messenger RNA (mRNA) samples ranged from 9.4 to 10 , and was similar between the MDD and CNTR groups and various conditions. The samples were stored at $-80^{\circ} \mathrm{C}$ until further use.

The mRNA levels in each fibroblast culture were examined with the GeneChip HT HG-U133+ PM Array Plate (Affymetrix, Santa Clara, CA, USA). The complementary DNA synthesis from total RNA, labeling and hybridization were performed according to manufacturer's protocol. The segmented images from each GeneChip were normalized and log2 transformed using GC-robust multi-array analysis. ${ }^{23}$ The average logarithmic ratio $\left(A L R=\right.$ Mean $_{M D D}-$ Mean $\left._{\mathrm{CNTR}}\right)$ was calculated for each gene probe, as an indicator for magnitude of change and Student's paired and group two-tailed $P$-values as indicators for significance of change. Significance was established according to the dual criteria of $|A L R|>0.3785(30 \%$ change) and both pairwise and groupwise $P \leqslant 0.01$. Quantitaive PCR validation of the microarray data was performed with $\mathrm{RT}^{2}$ custom Profiler PCR Arrays (Qiagen, Valencia, CA, USA), designed to test the mRNA expression level of 10 genes involved in the regulation of lipid metabolism: stearoyl-CoA desaturase (delta-9-desaturase), fatty acid binding protein 3, 7-dehydrocholesterol reductase, insulin induced gene 1, quinolinate phosphoribosyltransferase, 3-hydroxy-3-methylglutaryl-CoA reductase, 3hydroxy-3-methylglutaryl-CoA synthase 1, low-density lipoprotein receptor, fatty acid desaturase 1 , fatty acid desaturase 2 . The differentially expressed genes were subjected to a two-way hierarchical clustering analysis using GenePattern software. ${ }^{24}$ In addition, for each gene probe, we applied a linear statistical model with gene expression values as the outcome variable and group, stressor and group $\times$ stressor as fixed effects (groups: CNTR, MDD; stressors: GAL, RL). Data were analyzed with procedure MIXED in SAS software (version 9.3, SAS Institute, Cary, NC, USA). Gene set enrichment analysis (GSEA) based on pre-defined gene classes were carried out with the GenePattern software. ${ }^{25}$ BioCarta defined set of genes were considered significantly overrepresented at $P \leqslant 0.05$.

The miRNA levels were interrogated with miRNome miscript miRNA PCR Arrays (Qiagen). The complementary DNA was prepared from small RNA preps with miScript HiSpec Buffer according to miScript II RT Kit (Qiagen) instructions. The complementary DNA from each sample was quantified and proportionately combined into four pools of samples according to age and gender (Supplementary Material 1). Creating multiple sample pools from closely matched individuals allowed us a reduced work load and cost, while still retaining the ability to establish statistical significance in the downstream analyses. However, to avoid any pooling biases and outlier effects, the outcome of these data had to be validated in the individual samples. As a result, significantly changed miRNA levels in each individual fibroblast sample were examined with custom miScript miRNA PCR Arrays. (Qiagen), containing assays for the following miRNAs: miR-146b-5p, miR-550a, miR-214, miR-132*, miR-376c, miR-19a, miR-181a*, miR-486-5p, miR-424*, miR-542-3p, miR-22, miR-376b, miR-29b, miR-564. The selection of the 14 miRNA assays was based on a statistically significant difference in response to GAL or RL in the MDD or the CNTR group. The Ct values for each miRNA from both miRNome and custom $\mathrm{QPCR}$ arrays were normalized to the average $\mathrm{Ct}$ from a set of housekeeping genes and thus $\Delta \mathrm{Ct}$ values were generated. At $\mathrm{Ct}>35$, a miRNA level was considered nondetectable. In cases where the levels of a miRNA were non-detectable in two or more samples within a group, the miRNA was not used for further comparisons. The miRNA difference of expression between groups was measured by $\Delta \Delta \mathrm{Ct}_{\mathrm{RL}}=\mathrm{Mean}_{\mathrm{C}} \mathrm{Ct}_{\mathrm{RL}}-\mathrm{Mean}_{\mathrm{C}} \mathrm{Ct}_{\mathrm{STD}}$ and $\Delta \Delta \mathrm{Ct}_{\mathrm{GAL}}=\mathrm{Mean} \Delta \mathrm{Ct}$ $\mathrm{GAL}-\mathrm{Mean} \mathrm{Ct}_{\mathrm{STD}}$. A miRNA was considered differentially expressed when $\Delta \Delta \mathrm{Ct} \mid>0.3785$ and pairwise $P \leqslant 0.05$. Group $\times$ stressor interaction was determined by the dual criteria of $|\Delta \Delta \Delta C \mathrm{t}|>0.3785$ (30\% difference) and groupwise $\quad P \leqslant 0.05 . \quad \Delta \Delta \Delta \mathrm{Ct}_{\mathrm{GAL}}=\Delta \Delta \mathrm{Ct}_{\mathrm{MDDGAL}}-\Delta \Delta \mathrm{Ct}_{\mathrm{CNTRGAL}} ; \quad \Delta \Delta \Delta \mathrm{Ct}_{\mathrm{RL}}$ $=\Delta \Delta \mathrm{Ct}_{\mathrm{MDDRL}}-\Delta \Delta \mathrm{Ct}_{\mathrm{CNTRRL}}$

\section{RESULTS}

MDD fibroblast response to metabolic stress: mRNA changes

Metabolic stress response in MDD and CNTR fibroblasts from 32 subjects (16 MDD and 16 CNTR) was achieved by exposure to culture media containing GAL or RL (Supplementary Material 2). Analysis of mRNA species by oligonucleotide microarrays revealed that GAL substitution of glucose in the growth medium resulted in robust changes in the transcriptome of both MDD and CNTR fibroblasts. Using dual criteria for establishing significant expression differences ( $\mid$ ALR $\mathrm{GAL}_{\mathrm{STD}} \mid>0.3785$ (30\% change) and both group- and pairwise $P \leqslant 0.01$ ), we identified 1196 transcripts changed in GAL-treated MDD fibroblasts (Supplementary Material 3A). Of those, 975 were upregulated as a result of GAL treatment. According to the same criteria, 1111 probes were changed in CNTR fibroblasts, 733 of which were upregulated. In comparison with the CNTR fibroblasts, MDD fibroblasts revealed a distinct GAL-induced mRNA pattern; of the total number of mRNAs, $310(26 \%)$ were only exhibited in MDD (Supplementary Material 4A).

$\mathrm{RL}$ treatment also resulted in pronounced mRNA changes in both MDD and CNTR cells. Differential expression in RL conditions (compared with STD media) was noted for 312 transcripts in the MDD: 262 upregulated and 50 downregulated mRNAs. Similarly, in CNTR fibroblast, 360 mRNA species (261 upregulated, 99 downregulated) were differentially expressed in RL compared with STD conditions (Supplementary Material 3B). Importantly, the MDD fibroblasts responded to RL condition differently than CNTR; 103 (33.4\%) of all mRNA changes induced by RL were only exhibited in MDD (Supplementary Material 4B).

It was remarkable that both GAL and RL treatments resulted in distinct mRNA changes in MDD fibroblasts that were not present 
a MDD $\times$ GAL mRNA

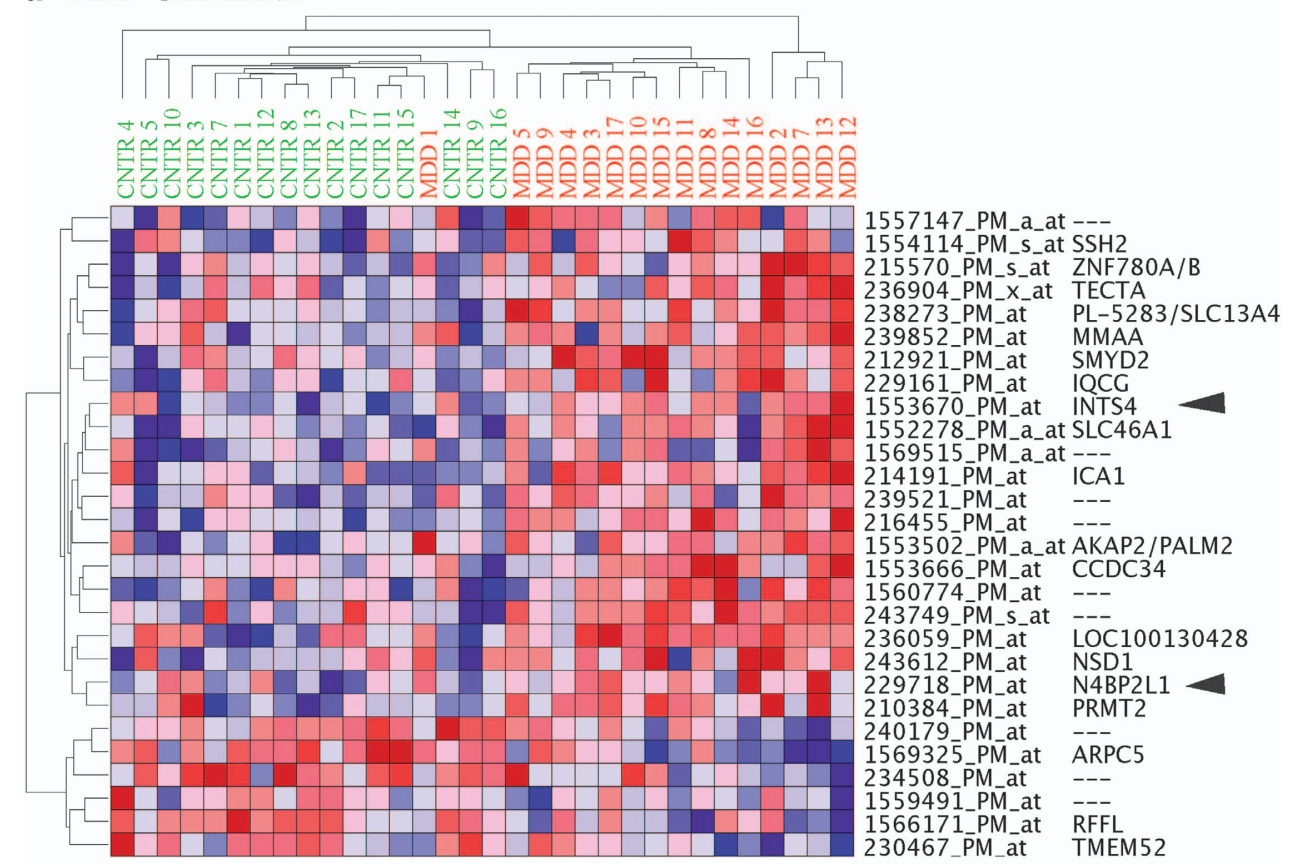

b MDD $\times$ RL mRNA

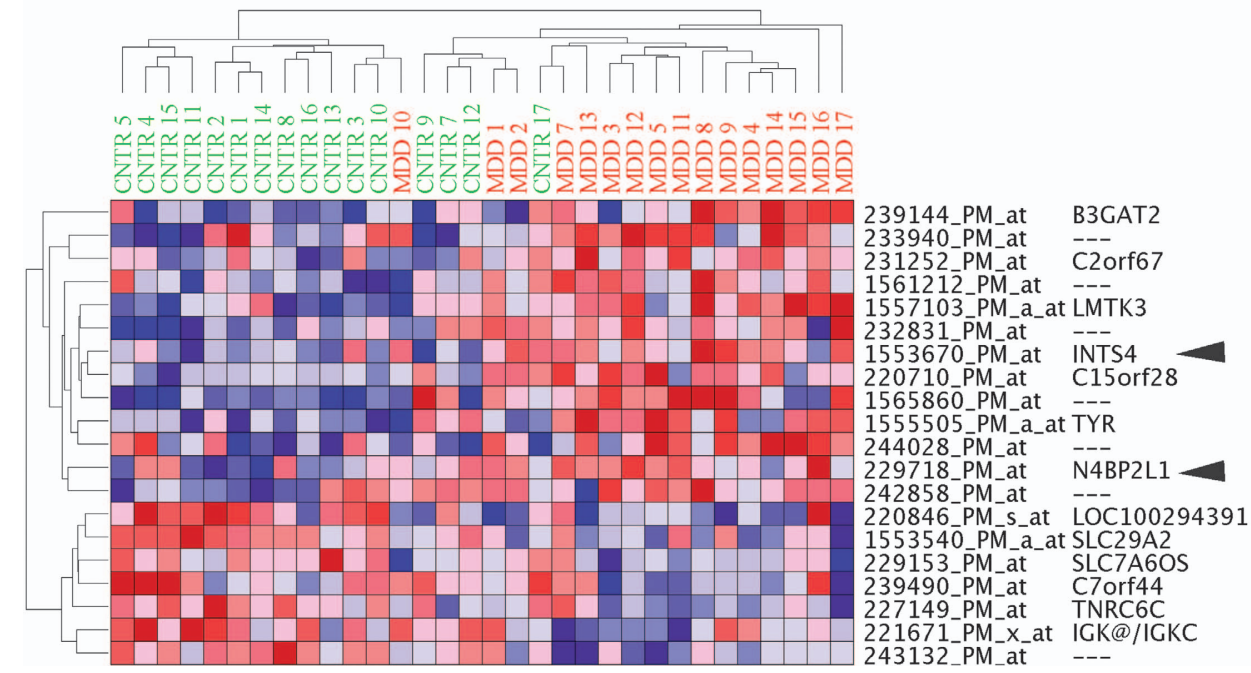

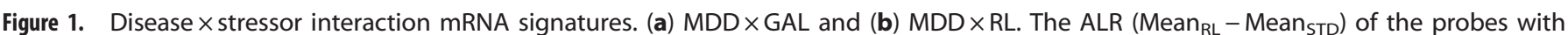
significant disease $\times$ challenge interaction were subjected to unsupervised hierarchical clustering. The colored squares represent the increase (red) or decrease (blue) of each ALR from the mean. Color intensity is proportional to magnitude of change. Clear separation of MDD and CNTR groups was observed. Furthermore, two genes (INTS4 and N4BP2L1-denoted by arrows) are commonly present in both signatures. CNTR, control; GAL, galactose; MDD, major depressive disorder; mRNA, messenger RNA; RL, reduced lipid; STD, standard.

in CNTR, therefore, we further sought to identify the interaction effect between disease state and metabolic challenge (group $x$ stressor) by applying a linear statistical model. We used a dual significance cutoff criterion of $|\Delta \mathrm{ALR}|>0.3785$ (30\% change) and $P \leqslant 0.01$. We found that the characteristic MDD signature in response to GAL (MDD $\times$ GAL) was defined by 28 (Figure $1 \mathrm{a})$, and in response to $\mathrm{RL}(\mathrm{MDD} \times \mathrm{RL})$ was defined by $20 \mathrm{mRNA}$ species (Figure 1b). Two of the genes (integrator complex subunit 4; INTS4 and NEDD4 binding protein 2-like 1; N4BP2L1) overlapped in the $M D D \times G A L$ and $M D D \times R L$ response-defining pattern.

Custom QPCR arrays were used to validate the microarraydetected mRNA changes in response to GAL and RL. The arrays were designed to test the mRNA expression level of 10 genes involved in lipid metabolism with significantly changed expression in MDD and in CNTR samples in GAL vs STD and in RL vs STD conditions. The statistical significance of the microarray-detected expression changes for all 10 genes was confirmed by qPCR (Figure 2). In addition, the microarray's ALRs had a high correlation with the qPCR's $-\triangle \Delta C t$ in both GAL vs STD and RL vs STD comparisons.

To better understand what molecular pathways are differently engaged in MDD compared with CNTR in response to metabolic stress, we performed GSEA with predetermined gene sets by BioCarta. Significantly enriched gene sets, representing molecular pathways, were identified for both MDD and CNTR in GAL and RL environment (Table 1, GSEA). GAL treatment induced enrichment of 21 pathways in MDD and 17 in CNTR. Nine of the 21 gene sets (43\%) were only enriched in MDD and are involved in cell cycle 


\begin{tabular}{|c|c|c|c|c|c|c|c|c|}
\hline \multirow[b]{4}{*}{ Gene } & \multicolumn{8}{|c|}{ CONTROL } \\
\hline & \multicolumn{4}{|c|}{ GALACTOSE } & \multicolumn{4}{|c|}{ REDUCED LIPID } \\
\hline & \multicolumn{2}{|l|}{ microarray } & \multicolumn{2}{|l|}{ qPCR } & \multicolumn{2}{|c|}{ microarray } & \multicolumn{2}{|l|}{ qPCR } \\
\hline & ALR(GAL-STD) & p-value & $-\Delta \Delta C t$ (GAL-STD) & p-value & ALR(RL-STD) & p-value & $-\Delta \Delta \mathrm{Ct}(\mathrm{GAL}-\mathrm{STD})$ & p-value \\
\hline SCD & 2.02 & 0.00000 & 2.66 & 0.00040 & 1.82 & 0.00000 & 2.23 & 0.00007 \\
\hline FABP3 & 1.86 & 0.00001 & 2.80 & 0.00397 & 1.89 & 0.00000 & 3.10 & 0.00014 \\
\hline DHCR7 & 1.18 & 0.00000 & 1.42 & 0.00021 & 1.82 & 0.00000 & 2.49 & 0.00000 \\
\hline INSIG1 & 1.43 & 0.00000 & 1.79 & 0.00571 & 1.61 & 0.00000 & 2.16 & 0.00004 \\
\hline QPRT & 1.42 & 0.00000 & 2.03 & 0.00125 & 1.96 & 0.00000 & 2.71 & 0.00036 \\
\hline HMGCR & 0.70 & 0.00017 & 0.43 & 0.64146 & 1.50 & 0.00000 & 1.18 & 0.19592 \\
\hline HMGCS1 & 1.17 & 0.00010 & 1.20 & 0.00937 & 1.64 & 0.00000 & 2.40 & 0.00000 \\
\hline LDLR & 1.08 & 0.00000 & 1.46 & 0.04054 & 1.55 & 0.00000 & 2.35 & 0.00020 \\
\hline FADS1 & 1.08 & 0.00000 & 1.36 & 0.00531 & 1.22 & 0.00000 & 1.57 & 0.00001 \\
\hline FADS2 & 1.00 & 0.00000 & 1.84 & 0.00143 & 1.51 & 0.00000 & 2.50 & 0.00000 \\
\hline
\end{tabular}

\begin{tabular}{|c|c|c|c|c|c|c|c|c|}
\hline \multirow[b]{4}{*}{ Gene } & \multicolumn{8}{|c|}{ MDD } \\
\hline & \multicolumn{4}{|c|}{ GALACTOSE } & \multicolumn{4}{|c|}{ REDUCED LIPID } \\
\hline & \multicolumn{2}{|l|}{ microarray } & \multicolumn{2}{|l|}{$\mathrm{qPCR}$} & \multicolumn{2}{|c|}{ microarray } & \multicolumn{2}{|l|}{$\mathrm{qPCR}$} \\
\hline & ALR(GAL-STD) & p-value & $-\Delta \triangle \mathrm{Ct}$ (GAL-STD) & p-value & ALR(RL-STD) & p-value & $-\Delta \triangle \mathrm{Ct}(\mathrm{GAL}-\mathrm{STD})$ & p-value \\
\hline SCD & 2.13 & 0.00001 & 2.37 & 0.00005 & 1.71 & 0.00000 & 2.21 & 0.00004 \\
\hline FABP3 & 1.88 & 0.00000 & 3.33 & 0.00028 & 1.55 & 0.00003 & 3.36 & 0.00003 \\
\hline DHCR7 & 1.24 & 0.00000 & 1.76 & 0.00172 & 1.99 & 0.00000 & 2.57 & 0.00000 \\
\hline INSIG1 & 1.44 & 0.00000 & 1.76 & 0.01071 & 1.59 & 0.00000 & 2.41 & 0.00008 \\
\hline QPRT & 1.67 & 0.00000 & 2.07 & 0.00313 & 2.02 & 0.00000 & 2.65 & 0.00043 \\
\hline HMGCR & 0.75 & 0.00004 & 0.98 & 0.01540 & 1.50 & 0.00000 & 1.96 & 0.00000 \\
\hline HMGCS1 & 1.21 & 0.00070 & 1.46 & 0.02899 & 1.73 & 0.00000 & 2.30 & 0.00011 \\
\hline LDLR & 1.24 & 0.00000 & 1.75 & 0.00665 & 1.70 & 0.00000 & 2.44 & 0.00002 \\
\hline FADS1 & 1.08 & 0.00003 & 1.36 & 0.00239 & 1.23 & 0.00000 & 1.58 & 0.00000 \\
\hline FADS2 & 0.76 & 0.00100 & 1.78 & 0.00342 & 1.27 & 0.00000 & 2.34 & 0.00010 \\
\hline
\end{tabular}

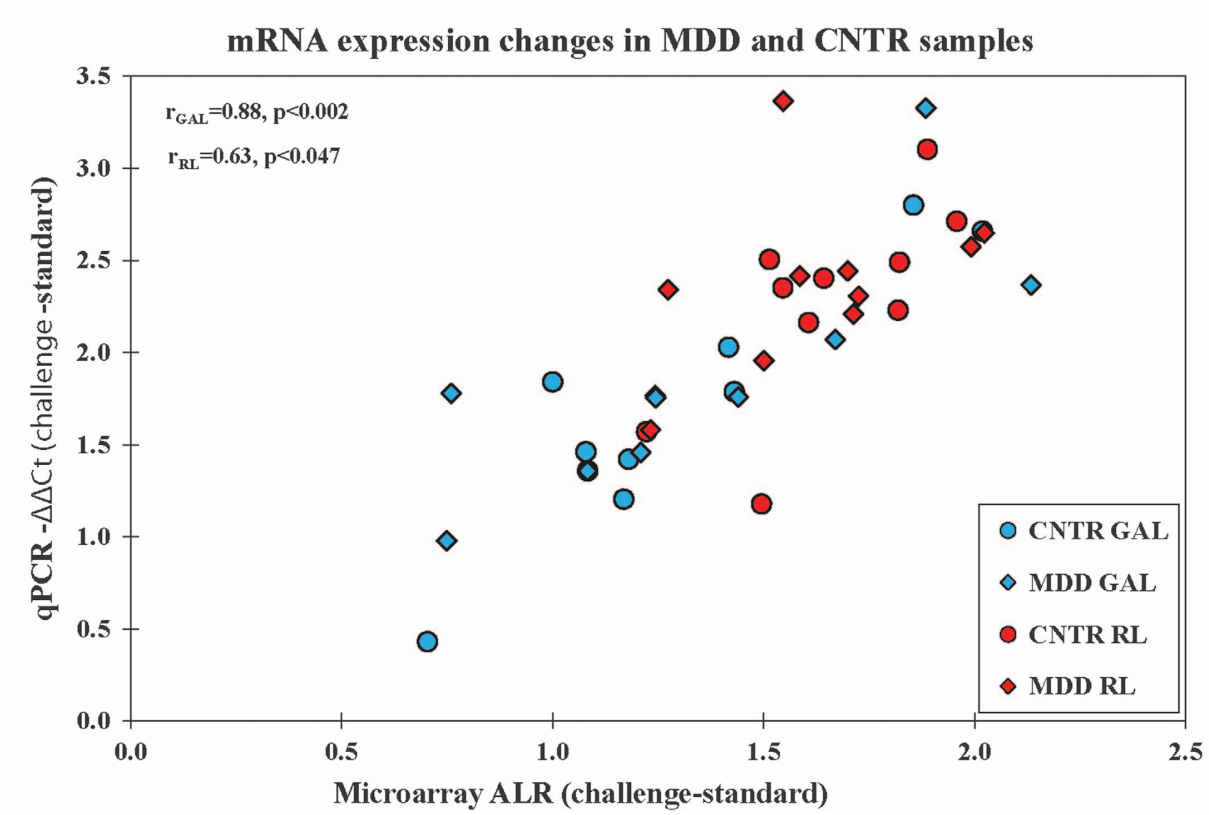

Figure 2. $\mathrm{qPCR}$ validation of the differential mRNA expression detected with microarrays. The differential expression of $10 \mathrm{mRNAs}$, detected with microarrays, was validated with custom qPCR arrays (groups: MDD, CNTR; culture conditions: STD, GAL, RL). ALR (ALR $\mathrm{GAL}_{\mathrm{L}}=\mathrm{Mean}_{\mathrm{GAL}}-$ Mean $_{S T D}$, blue, $A L R_{R L}=$ Mean $_{R L}-$ Mean $_{S T D}$, pink) was used as an estimate for the microarray expression changes and was plotted on the $x$ axis. $\Delta \Delta \mathrm{Ct}\left(\Delta \Delta \mathrm{Ct}_{\mathrm{GAL}}=\Delta \mathrm{Ct}_{\mathrm{GAL}}-\Delta \mathrm{Ct}_{\mathrm{STD}}\right.$, blue; $\Delta \Delta \mathrm{Ct}_{\mathrm{RL}}=\Delta \mathrm{Ct}_{\mathrm{RL}}-\Delta \mathrm{Ct}_{\mathrm{STD}}$, pink) was used for a $\mathrm{qPCR}$ expression changes estimate and was plotted as $-\Delta \Delta \mathrm{Ct}$ on the $\mathrm{y}$ axis. Note that the estimates from both analyses in each comparison were highly correlated. CNTR, control; GAL, galactose; MDD, major depressive disorder; mRNA, messenger RNA; qPCR, quantitative PCR; RL, reduced lipid; STD, standard.

regulation (PTC1, MPR, ACAP95, ARF), apoptosis (HSP27, SET, BAD), anti-inflammation (IL10) and cell survival (IL7). RL resulted in enrichment of 19 pathways in MDD and 15 in CNTR. Ten of the 19 gene sets (53\%) were only enriched in MDD and are involved in cell repair (TFF), survival (TRKA), migration (ECM) and proliferation (CDMAC, SPRY), and also in regulation of metabolism $(\mathrm{GH})$ and energy production (FEEDER, GLYCOLYSIS). Overall, GSEA revealed that the specific changes in MDD evoked by both metabolic stressors are represented by approximately half of all detected molecular pathways, indicating that MDD fibroblasts utilize different strategies for adaptation to the metabolic stressors. Moreover, challenging the MDD fibroblasts with GAL and RL exposed characteristic molecular signatures undetectable at STD conditions.
MDD fibroblast response to metabolic stress: miRNA changes

The miRNAs are important regulators of mRNA function and they are heavily involved in control of metabolism and energy

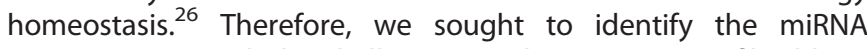
response to metabolic challenges in the same MDD fibroblasts in which differential mRNA expression patterns had been established.

The miRNA samples, isolated individually from the 16 MDD and 16 CNTR fibroblast cultures, were pooled into four sets according to the patients' age and gender (Supplementary Material 1). Human miRNome miScript miRNA PCR Arrays (Qiagen) were used to profile the expression of the 1008 most abundantly expressed miRNA species in the human miRNA genome (miRNome). 
Table 1. BioCarta GSEA enrichment of mRNA expression in response to $\mathrm{GAL}$ and $\mathrm{RL}$ challenges

\begin{tabular}{|c|c|c|c|}
\hline Pathway & Size & NES & P-value \\
\hline \multicolumn{4}{|l|}{ Enriched in CNTR GAL } \\
\hline RACCYCD & 26 & 1.89 & 0.0076 \\
\hline CELLCYCLE & 23 & 1.81 & 0.0000 \\
\hline TEL & 18 & 1.74 & 0.0040 \\
\hline SRCRPTP & 11 & 1.72 & 0.0000 \\
\hline P53 & 16 & 1.66 & 0.0078 \\
\hline CERAMIDE & 22 & 1.65 & 0.0115 \\
\hline CFTR & 12 & 1.62 & 0.0000 \\
\hline CHREBP2 & 42 & 1.61 & 0.0275 \\
\hline G2 & 24 & 1.61 & 0.0199 \\
\hline ATRBRCA & 21 & 1.58 & 0.0159 \\
\hline CARM_ER & 34 & 1.58 & 0.0373 \\
\hline STATHMIN & 19 & 1.56 & 0.0233 \\
\hline ATM & 20 & 1.55 & 0.0492 \\
\hline CHEMICAL & 22 & 1.53 & 0.0497 \\
\hline $\mathrm{RB}$ & 13 & 1.53 & 0.0346 \\
\hline AKAPCENTROSOME & 15 & 1.52 & 0.0471 \\
\hline PLCE & 12 & 1.51 & 0.0299 \\
\hline \multicolumn{4}{|l|}{ Enriched in CNTR RL } \\
\hline PPARA & 56 & 1.83 & 0.0000 \\
\hline RACCYCD & 26 & 1.71 & 0.0229 \\
\hline CHREBP2 & 42 & 1.65 & 0.0136 \\
\hline CD40 & 15 & 1.59 & 0.0301 \\
\hline ARF & 17 & 1.57 & 0.0359 \\
\hline VIP & 26 & 1.57 & 0.0169 \\
\hline P38MAPK & 39 & 1.53 & 0.0076 \\
\hline HSP27 & 15 & 1.50 & 0.0389 \\
\hline BAD & 26 & 1.50 & 0.0217 \\
\hline GCR & 19 & 1.47 & 0.0360 \\
\hline LYM & 11 & 1.43 & 0.0368 \\
\hline PLCE & 12 & 1.43 & 0.0443 \\
\hline IL22BP & 16 & -1.53 & 0.0300 \\
\hline DNAFRAGMENT & 10 & -1.54 & 0.0237 \\
\hline AHSP & 11 & -1.56 & 0.0412 \\
\hline \multicolumn{4}{|l|}{ Enriched in MDD GAL } \\
\hline TEL & 18 & 1.98 & 0.0000 \\
\hline SRCRPTP & 11 & 1.73 & 0.0020 \\
\hline PTC1 & 11 & 1.73 & 0.0000 \\
\hline G2 & 24 & 1.66 & 0.0021 \\
\hline CARM_ER & 34 & 1.66 & 0.0056 \\
\hline HSP27 & 15 & 1.65 & 0.0116 \\
\hline $\mathrm{RB}$ & 13 & 1.62 & 0.0062 \\
\hline CERAMIDE & 22 & 1.60 & 0.0212 \\
\hline P53 & 16 & 1.58 & 0.0220 \\
\hline SET & 11 & 1.58 & 0.0328 \\
\hline CHEMICAL & 22 & 1.58 & 0.0243 \\
\hline ATRBRCA & 21 & 1.57 & 0.0080 \\
\hline MPR & 34 & 1.56 & 0.0413 \\
\hline CELLCYCLE & 23 & 1.56 & 0.0305 \\
\hline AKAP95 & 12 & 1.56 & 0.0469 \\
\hline CHREBP2 & 42 & 1.56 & 0.0222 \\
\hline IL10 & 17 & 1.53 & 0.0320 \\
\hline ARF & 17 & 1.50 & 0.0382 \\
\hline PLCE & 12 & 1.47 & 0.0301 \\
\hline IL7 & 17 & 1.46 & 0.0258 \\
\hline BAD & 26 & 1.43 & 0.0138 \\
\hline \multicolumn{4}{|l|}{ Enriched in MDD RL } \\
\hline RACCYCD & 26 & 1.90 & 0.0040 \\
\hline TFF & 21 & 1.88 & 0.0064 \\
\hline CD40 & 15 & 1.78 & 0.0102 \\
\hline TRKA & 12 & 1.74 & 0.0084 \\
\hline ECM & 24 & 1.67 & 0.0234 \\
\hline PPARA & 56 & 1.63 & 0.0066 \\
\hline HSP27 & 15 & 1.62 & 0.0176 \\
\hline GH & 28 & 1.60 & 0.0204 \\
\hline GLEEVEC & 23 & 1.58 & 0.0361 \\
\hline
\end{tabular}

Table. 1. (Continued)

\begin{tabular}{lccc}
\hline Pathway & Size & NES & P-value \\
\hline ARF & 17 & 1.54 & 0.0463 \\
EXTRINSIC & 13 & 1.53 & 0.0140 \\
CHREBP2 & 42 & 1.53 & 0.0246 \\
CDMAC & 16 & 1.52 & 0.0355 \\
BAD & 26 & 1.48 & 0.0118 \\
SPRY & 18 & 1.45 & 0.0417 \\
FEEDER & 9 & -1.53 & 0.0236 \\
DNAFRAGMENT & 10 & -1.54 & 0.0488 \\
AHSP & 11 & -1.56 & 0.0354 \\
GLYCOLYSIS & 10 & -1.63 & 0.0040 \\
\hline
\end{tabular}

Abbreviations: CNTR, control; GAL, galactose; GSEA, gene set enrichment analysis; MDD, major depressive disorder; mRNA, messenger RNA; RL, reduced lipid. Molecular pathway enrichment of mRNA in MDD and CNTR. Pathways enriched only in one group (MDD or CNTR) are in bold.

Approximately $50 \%$ of the assayed miRNAs were detected in human cultured dermal fibroblasts, regardless of disease status or culture condition. Significant expression differences in metabolic stress vs STD conditions were defined when change was larger than $30 \%(|\Delta \Delta \mathrm{Ct}|>0.3785)$ and paired $t$-test $P \leqslant 0.05$. In response to GAL challenge, MDD fibroblasts revealed expression changes in 63 miRNAs (Supplementary Material 5A). The number of miRNAs changed by GAL challenge in CNTR samples was substantially lower, 38 miRNAs. In addition, 81\% of the miRNAs changed in GAL were specific for MDD fibroblasts.

RL elicited much stronger miRNA response in MDD (147 miRNAs), compared with CNTR (38 miRNA) (Supplementary Material 5B), leading us to believe that MDD fibroblasts are experiencing the RL environment as much more stressful than CNTRs. Furthermore, $90 \%$ of the miRNA RL-response pattern was observed only in MDD, but not in CNTR.

Validation of miRNome analyses on pooled samples was performed with custom qPCR array of 14 miRNAs (SABiosciences, Qiagen) on individual samples. The $\Delta \Delta \mathrm{Ct}\left(\Delta \Delta \mathrm{Ct}_{\mathrm{GAL}}=\Delta \mathrm{Ct}_{\mathrm{GAL}}-\Delta \mathrm{Ct}\right.$ STD, $\Delta \Delta \mathrm{Ct}_{\mathrm{RL}}=\Delta \mathrm{Ct}_{\mathrm{RL}}-\Delta \mathrm{Ct}_{\mathrm{STD}}$ ) from the pooled samples for each miRNA was correlated with the corresponding $\Delta \Delta C t$ from the individual samples (Figure 3). Both GAL- and RL-induced miRNA response demonstrated high correlation between pooled and individual samples with coefficients of $r_{\mathrm{GAL}}=0.920$ and $r_{\mathrm{RL}}=0.785$. In addition, $85 \%$ of the miRNA changes in the individual samples were significantly different in the predicted direction $(P \leqslant 0.05)$, providing a strong validation for the miRNome data.

In addition, in an attempt to identify the miRNA changes that characterize the specific response of MDD to each metabolic challenge, we used a group $\times$ challenge linear statistical model and the dual significance criterion of $|\Delta \Delta \Delta C \mathrm{t}|>0.3785$ and $P \leqslant 0.05$. Thus, we derived two miRNA signatures: (1) representing MDD $\times$ GAL interaction (16 miRNAs) and (2) representing MDD $\times$ RL interaction (36 miRNAs, Figure 4). It is noteworthy that miR-7, miR-382, miR-296-5p and miR-3176 were common for both signatures.

Literature search in PubMed for the roles of the MDD $\times G A L$ and $M D D \times R L$ signature miRNAs revealed an interesting trend toward extensive miRNA involvement in the regulation of metabolism, cell proliferation, survival and migration (Table 2 ). In relation to control of metabolism, we found miRNAs regulating insulin levels and secretion, obesity and starvation in both MDD $\times$ GAL (38\%) and $\mathrm{MDD} \times \mathrm{RL}(37 \%)$ panels. In addition, eight miRNAs from the MDD $\times$ RL panel: miR-3613-3p, miR-33a, miR-192, miR-26a, miR-34a, miR-370, miR-15b and miR-296-5p, have been described in the literature as regulators of lipid metabolism. With regard to cell proliferation and apoptosis, $56 \%$ of the MDD $\times$ GAL and $61 \%$ of the $M D D \times R L$ miRNAs are known controllers of various signaling 


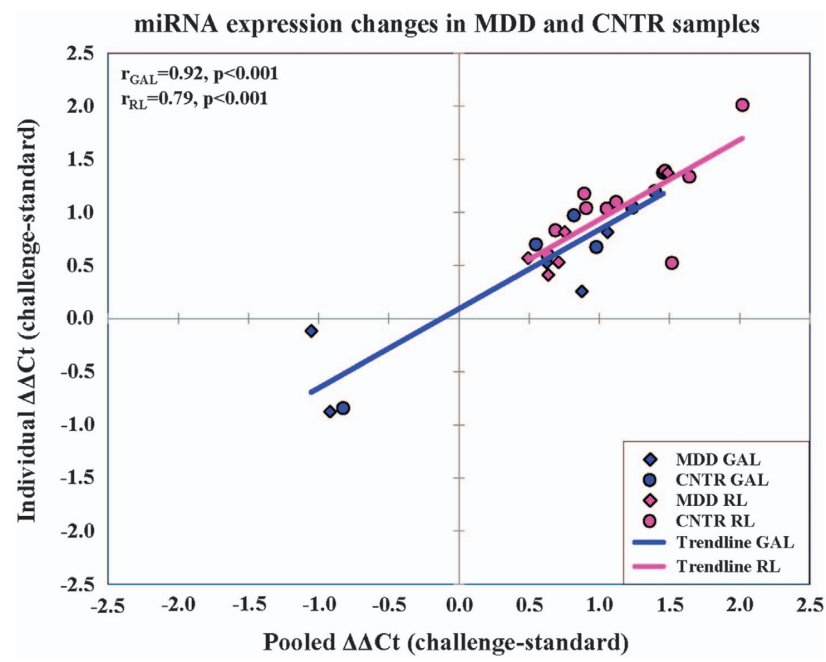

Figure 3. Differential miRNA expression in pooled samples is validated in individual samples. The expression level of 14 miRNAs, detected in miRNome analyses of pooled samples, was validated with independent qPCR arrays of individual samples (groups: MDD, CNTR; culture conditions: STD, GAL, RL). The expression changes, estimated with $\Delta \Delta \mathrm{Ct}\left(\Delta \Delta \mathrm{Ct}_{\mathrm{GAL}}=\Delta \mathrm{Ct}_{\mathrm{GAL}}-\Delta \mathrm{Ct}_{\mathrm{STD}}\right.$, blue; $\Delta \Delta \mathrm{Ct}_{\mathrm{RL}}$ $=\Delta \mathrm{Ct}_{\mathrm{RL}}-\Delta \mathrm{Ct}_{\mathrm{STD}}$, pink), from the pooled samples are plotted on the $x$ axis, and from the individual samples on the $y$ axis. Note that the values from each comparison were highly correlated. CNTR, control; GAL, galactose; MDD, major depressive disorder; miRNA, microRNA; qPCR, quantitative PCR; RL, reduced lipid; STD, standard.

cascades, out of which $13 \%(\mathrm{GAL})$ and $33 \%$ (RL) affect or are affected by the tumor suppressor protein p53. As p53 is not only a modulator of apoptosis, but is also involved in regulation of metabolism, ${ }^{27}$ some overlap between these categories is expected. Furthermore, 25\% (MDD $\times \mathrm{GAL})$ and 39\% (MDD $\times \mathrm{RL})$ of the signature miRNAs are known to regulate cell motility. Interestingly, three of them: miR-34a, miR-34b and miR-199a-3p, are recognized as regulators of the hepatocyte growth factor receptor, c-Met, ${ }^{28,29}$ a key controller of cell migration.

\section{DISCUSSION}

Our results demonstrate that the metabolic challenges evoked by substitution of glucose with GAL or reducing the abundance of lipids in the growth media of fibroblast cultures result in robust changes of mRNA and miRNA expression. MDD fibroblasts responded to the metabolic stress with alternate expression of 1196 (GAL) and 312 (RL) transcripts. A substantial fraction of these mRNA changes, 26\% (GAL) and 33\% (RL) were only observed in MDD, but not in CNTR. The specific response of MDD fibroblasts to each challenge was represented by 20 (MDD $\times \mathrm{GAL})$ and 28 $(M D D \times R L) m R N A s$ with significant group by challenge interaction. The characteristic response of MDD fibroblasts to the metabolic stressors was even more pronounced on the miRNA level; $81 \%$ $(G A L)$ and $90 \%(R L)$ of the total miRNA changes were only observed in MDD, but not in CNTR. The miRNA signature response of MDD fibroblasts was defined by $16(\mathrm{MDD} \times \mathrm{GAL})$ and 36 $(\mathrm{MDD} \times \mathrm{RL}) \mathrm{miRNAs}$. The stress-induced changes, observed on both mRNA and miRNA level, revealed MDD-associated impairments in molecular pathways involved in the control of metabolism and energy production, cell survival, proliferation and migration. These impairments are likely to be present in all the somatic cells, and therefore are informative of the disease process. In fact, the results are consistent with our previous findings in human postmortem brain tissue, as both studies implicated disturbances in apoptotic molecular pathways. ${ }^{30}$ Impairments in any of the detected molecular pathways could be critical for the development and plasticity of the brain and can result in the disease state of MDD. ${ }^{31}$ In a previous study, MDD fibroblasts cultured in STD condition revealed deficits in cell proliferation and cell motility. ${ }^{32}$ In this study, we demonstrated that molecular pathways involved in the same processes were also impaired in response to metabolic challenges with GAL and RL, and revealed previously unknown disruption of pathways regulating metabolism and energy production. Presenting metabolic challenges was instrumental for defining distinct MDD mRNA and miRNA response signatures that might also contribute to patients' aberrant responses to life stressors.

GAL and RL treatments elicited common responses in MDD fibroblasts. The commonality was demonstrated on both mRNA and miRNA level. On mRNA level, the overlap between MDD $\times G A L$ and $\mathrm{MDD} \times \mathrm{RL}$ signatures was defined by the integrator complex subunit 4 (INTS4), and the NEDD4 binding protein 2-like 1 (N4BP2L1). The INTS4 associates with RNA polymerase II, and therefore potentially has a general effect on regulation of gene transcription and RNA processing. ${ }^{33}$ The N4BP2L 1 is a paralog of N4BP2 and is likely involved in cell survival. ${ }^{34}$ Evidence for MDD impairment in the regulation of cell survival and proliferation streamed from the molecular pathway analyses as well. The HSP27, BAD and ARF molecular pathways were the commonly enriched pathways in MDD during GAL and RL challenges; all of which affect cell survival and proliferation. Furthermore, 38\% (GAL) and $17 \%(R L)$ of the MDD-enriched gene sets (PTC1, MPR, ACAP95, ARF, HSP27, SET, BAD, IL7, TRKA, CDMAC, SPRY), contribute to the regulation of cell survival and proliferation. On the miRNA level, three of the commonly changed miRNAs are regulators of cell proliferation and apoptosis. ${ }^{35-37}$ In addition, a striking $56-61 \%$ of the miRNAs from the MDD $\times$ GAL and $\mathrm{MDD} \times \mathrm{RL}$ signatures have roles in cell cycle and survival regulation. Overall, these data provide an overwhelming support for the aberrant engagement of mechanisms regulating cell proliferation and survival by MDD fibroblasts when exposed to two distinct metabolic challenges. Commonality between GALand RL-induced responses in MDD was also detected in their respective miRNA signatures, where at least 38\% (GAL) and 37\% $(\mathrm{RL})$ of the miRNAs were regulators of metabolism or energy homeostasis, indicating deficiencies in those signaling cascades in MDD as well. In summary, the challenges presented by GAL and RL in MDD fibroblasts affected cellular processes, such as control of cell proliferation and survival, regulation of metabolism and energy production. The molecular machinery for regulation of these processes is located mainly in the mitochondria. Therefore, our data strongly support the disruption of mitochondrial functions in patients with MDD, as previously suggested. ${ }^{10}$

The GAL and RL treatment also evoked different responses in MDD fibroblasts. The number of mRNAs with changed expression in GAL was four times the number of mRNAs changed in RL. In contrast, the mRNA signatures characterizing the specific response of MDD to each stress, have similar number of mRNAs (MDD $\times$ $\mathrm{GAL}=28, \mathrm{MDD} \times \mathrm{RL}=20$ ). Therefore, the proportion of the specific mRNAs from the total mRNA changes $(G A L=1196, R L=360)$ is in favor of RL (5.6 vs $2.3 \%$ ). Consequently, we concluded that RL vs GAL environment more strongly illuminates the molecular deficits of MDD. Furthermore, additional data, obtained from RL treatment, reveal abnormalities in MDD, which were not apparent in GAL. These are the eight miRNAs in the MDD $\times$ RL miRNA signature known to control lipid metabolism, pointing toward the possibility of fatty acid metabolism dysregulation in patients with MDD, a phenomenon studied in many different ways during the last couple of decades. ${ }^{38-42}$ Another interesting fact revealed only in $\mathrm{RL}$ environment is the presence of six hypoxamirs in the $M D D \times R L$ miRNA signature. Hypoxamirs have been identified as miRNAs contributing to mitochondrial respiration arrest in a low oxygen environment. ${ }^{43}$ In our study, CNTR fibroblasts upregulated six hypoxamirs (let-7b*, miR-192, miR-26a, miR-98, miR-23a and 


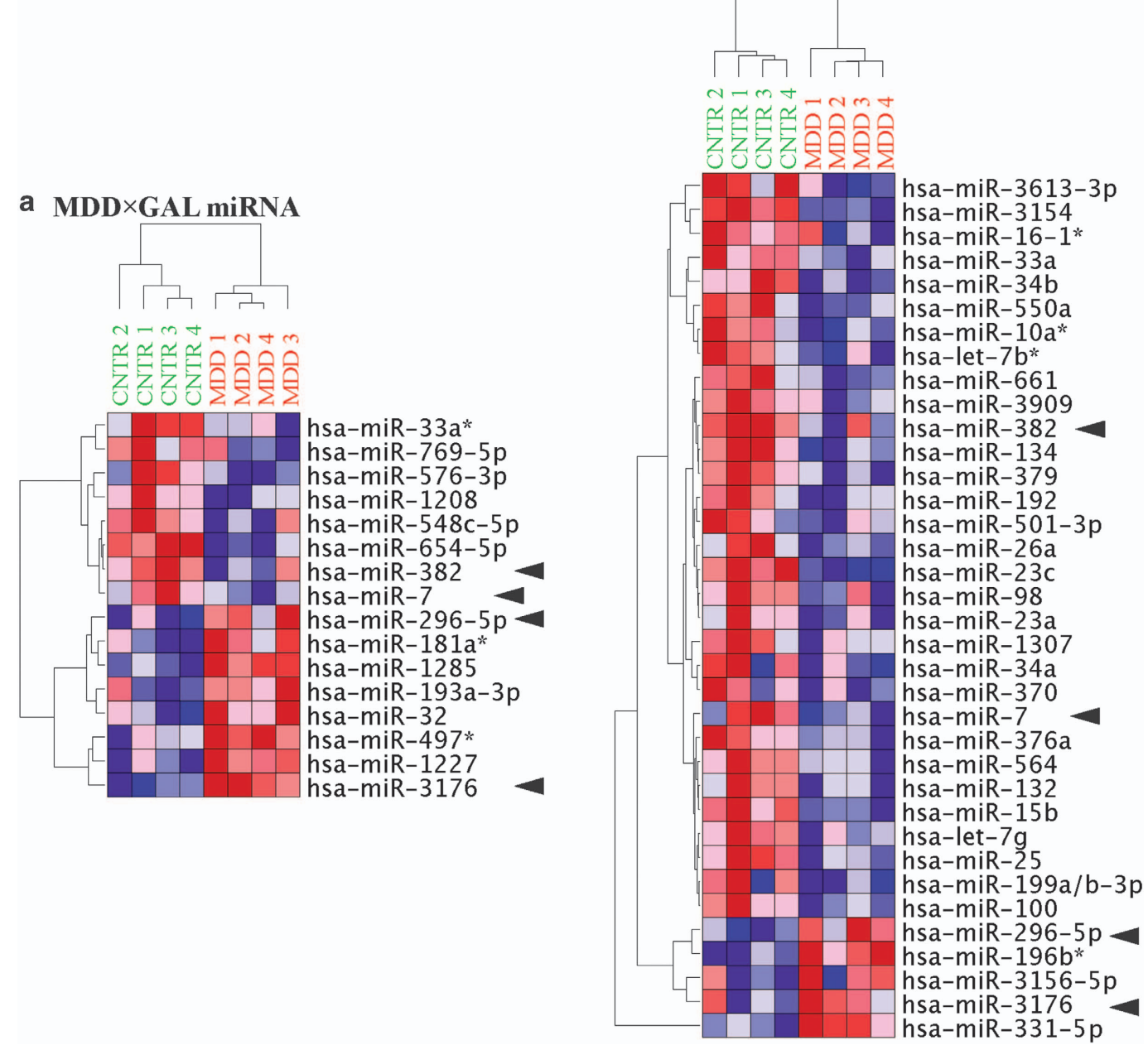

\section{b MDD $\times$ RL miRNA}

Figure 4. Disease $\times$ stressor interaction miRNA signatures. (a) MDD $\times \mathrm{GAL}$ and (b) MDD $\times \mathrm{RL}$. The $\Delta \Delta \mathrm{Cts}\left(\Delta \Delta \mathrm{Ct}_{\mathrm{GAL}}=\Delta \mathrm{Ct}_{\mathrm{GAL}}-\Delta \mathrm{Ct}_{\mathrm{STD}} ; \Delta \mathrm{Ct}_{\mathrm{RL}}\right.$ $\left.=\Delta \mathrm{Ct}_{\mathrm{RL}}-\Delta \mathrm{Ct}_{\mathrm{STD}}\right)$ of the miRNAs with significant disease $\times$ challenge interaction were subjected to unsupervised hierarchical clustering. The colored squares represent the increase (red) or decrease (blue) of each $\Delta \Delta \mathrm{Ct}$ from the mean. Increased $\Delta \Delta \mathrm{Ct}$ represents reduced level of miRNA in metabolic stress compared with STD conditions. Clear separation of MDD and CNTR groups was observed. Four miRNAs (that is, miR-7, miR-382, miR-296-5p and miR-3176), denoted by arrows, are commonly present in both signatures. CNTR, control; GAL, galactose; MDD, major depressive disorder; miRNA, microRNA; RL, reduced lipid; STD, standard.

$\left.\mathrm{miR}^{-7}\right)^{44}$ in response to $\mathrm{RL}$, whereas MDD fibroblasts downregulated them. This particular difference in the MDD response compared with CNTR further supports the notion of mitochondrial dysfunction in MDD. The RL challenge was also very informative in uncovering abnormalities in the process of cell migration; $39 \%$ of the miRNAs in the MDD $\times R L$ miRNA signature were regulators of metastasis. Also, MDD responded to $\mathrm{RL}$ with enrichment of ECM (extracellular matrix) pathway and TFF (Trefoil factors initiate mucosal healing) pathway; both important players in the processes of cell migration. The deficit of MDD in cell migration was not as apparent during adaptation to GAL, but was observed in a previous study in STD conditions. ${ }^{32}$

An important question is whether the changes we detect in peripheral tissues are relevant to a disease process that takes place primarily in the brain. Genetic factors associated with MDD are likely to be present throughout the entire body and not just be specific to the brain. The adaptive deficits detected in fibroblast cell lines can be linked back to the function of neurons and the brain, with multiple examples found in the mRNA and miRNA challenge-response signatures and in the altered molecular pathways. On mRNA level, such examples are presented by genes important for neurite outgrowth, neurodevelopment and synaptic plasticity: Actin-related protein 2/3 complex, subunit 5 (ARPC5), ${ }^{45}$ ArfGAP with coiled-coil, ankyrin repeat and $\mathrm{PH}$ domains 2 $\left(\right.$ ACAP2) ${ }^{46}$ and Beta-1,3-glucuronyltransferase 2 (glucuronosyltransferase S, B3GAT2). ${ }^{47}$ Multiple important roles in the nervous system are associated with miRNA challenge-response signatures as well. Generally, $31 \%$ (GAL) and $47 \%$ (RL) of the miRNAs from the miRNA signatures are either regulators of molecular events in the brain or biological markers associated with psychiatric disorders in the periphery. In particular, we identified miRNAs with roles in neurite outgrowth and synapse formation: miR-7, ${ }^{48}$ miR-26a, ${ }^{49}$ miR-34a, ${ }^{50}$ miR-134 (ref. 51) and miR-132, ${ }^{52,53}$ cell cycle progression, apoptosis and specification of neurons: miR-25, ${ }^{54}$ miR-34a, ${ }^{55}$ miR-497 (ref. 56) and miR-376a; brain morphogenesis: miR-7, ${ }^{58}$ miR-370 (ref. 59) and myelination in the central nervous system: miR-23a. ${ }^{60}$ Furthermore, our study identified miRNAs implicated in the pathological development of schizophrenia, such as miR-132 (ref. 61) and miR-382; ${ }^{62}$ and of Alzheimer's, such as miR-98. ${ }^{63}$ In addition, several of the miRNAs with aberrant challenge-response in MDD have been described as potential peripheral biomarkers for psychiatric or neurological diseases: miR-564 (ref. 64) and miR-132 for schizophrenia; ${ }^{65}$ hsa-let-7g and miR-15b for Alzheimer's; ${ }^{66}$ and miR-1285, miR-34a and miR-34b for Huntington's 
Table 2. Experimentally established involvement of the miRNAs from MDD $\times G A L$ and $M D D \times R L$ signatures in cellular processes (on the basis of PubMed search)

\begin{tabular}{|c|c|}
\hline Physiological process & miRNA_ID \\
\hline \multicolumn{2}{|l|}{$M D D \times G A L$ MiRNA } \\
\hline Metabolism & miR-33a*, miR-7, miR-296-5p, miR-181a*, miR-497* \\
\hline Fatty acid metabolism & miR-296-5p, miR-193a-3px \\
\hline P53 & miR-1208, miR-1285, miR-7 \\
\hline Apoptosis/proliferation & miR-548c-5p, miR-7, miR-382, miR-296-5p, miR-181*, miR-193a-3p, miR-1285, miR-32, miR-497* \\
\hline Migration/c-MET & miR-548c-5p, miR-7, miR-296-5p, miR-497* \\
\hline Neurons/psychiatry & miR-7, miR-382, miR-296-5p, miR-32, miR-497* \\
\hline \multicolumn{2}{|r|}{ - } \\
\hline Metabolism & $\begin{array}{l}\text { miR-33a, miR-7, miR-661, miR-1307, miR-376a, miR-26a, miR-132, miR-25, miR-98, miR-34a, miR-23a, miR-199a/b-3p, } \\
\text { miR-296-5p }\end{array}$ \\
\hline Fatty acid metabolism & miR-3613-3p, miR-33a, miR-192, miR-26a, miR-34a, miR-370, miR-15b, miR-296-5p \\
\hline P53 & miR-34b, miR-16-1*, miR-661, miR-192, miR-7, miR-134, miR-25, miR-98, miR-34a, miR-370, miR-15b, miR-199a/b-3p \\
\hline Apoptosis/proliferation & $\begin{array}{l}\text { miR-33a, miR-34b, miR-16-1*, miR-661, miR-192, miR-7, miR-376a, miR-382, miR-26a, miR-134, miR-379, let-7g, miR-132, } \\
\text { miR-25, miR-98, miR-34a, miR-370, miR-15b, miR-23a, miR-199a/b-3p, miR-100, miR-296-5p }\end{array}$ \\
\hline Migration/c-MET & $\begin{array}{l}\text { miR-34b, miR-661, miR-550a, miR-7, miR-376a, miR-26a, let-7g, miR-134, miR-132, miR-25,miR-98, miR-34a, miR-100, } \\
\text { miR-296-5p }\end{array}$ \\
\hline Neurons/psychiatry & $\begin{array}{l}\text { miR-34b, miR-7, miR-376a, miR-382, miR-26a, miR-134, miR-564, let-7g, miR-132, miR-25, miR-98, miR-34a, miR-370, } \\
\text { miR-15b, miR-23a, miR-199a/b-3p, miR-296-5p }\end{array}$ \\
\hline
\end{tabular}

Abbreviations: GAL, galactose; MDD, major depressive disorder; miRNA, microRNA; RL, reduced lipid. Metabolism/fatty acid metabolism. ${ }^{36,63,74-94}$ Apoptosis/ proliferation/p53..$^{37,49,54,56,58,95-118}$ Migration. ${ }^{29,54,98,99,119-131}$ Neurons/psychiatry. ${ }^{48-51,53,54,56-58,60,661,63-66,68-71,98,132-134}$

disease. $^{67}$ This study also identified a number of miRNAs that respond to different stressors in the nervous system: miR-34b, which affects the degree of oxidative stress and survival of dopaminergic neurons; ${ }^{68}$ miR-296-5p, which responds to oxidative stress in mouse hippocampal neuronal cultures; ${ }^{69}$ miR-199a, which reacts to hypoxia in rat cortical pericytes; ${ }^{70}$ and miR-98, which is elevated in the brains of newborn rats experiencing prenatal stress. ${ }^{71}$ And last, miR-296-5p, which showed aberrant response to both RL and GAL in MDD fibroblasts, presents a strong connection to MDD; miR-296-5p has been identified as a regulator of inducible I kappa-B kinase, $^{72}$ a gene with genetic association with MDD. ${ }^{73}$

Taken together, this information gives confidence to the relevance of our observations in fibroblasts to the MDD disease state. This study provides a solid foundation for future experiments with human fibroblasts aimed at deciphering the systemic immune disturbances in MDD. Moreover, our results demonstrate that aberrant responses to stressors are indeed present in the peripheral tissues from MDD patients. These responses intersect multiple cellular processes associated with mitochondria, such as regulation of metabolism and energy production, cell proliferation, survival and motility; therefore, they strongly suggest that the stress-response impairments of MDD patients are most likely based on impaired mitochondrial functions.

\section{CONFLICT OF INTEREST}

RCS receives unrelated research support from Assurex Health; Bristol-Myers Squibb; Eli Lilly and Company; Elan Corporation; Euthymics Bioscience; Forest Pharmaceuticals; Janssen Pharmaceutica; Jazz Pharmaceuticals; Naurex; Novartis Pharmaceuticals; Otsuka Pharmaceuticals; Pamlab; Pfizer; Repligen Corporation; Ridge Diagnostics; St. Jude Medical; and Takeda Pharmaceuticals and consults for Bristol-Myers Squibb Company; Cerecor; Cyberonics; Eli Lilly and Company; Forest Pharmaceuticals; Janssen Pharmaceutica; Medtronic; Naurex; Pamlab; Pfizer; Ridge Diagnostics; Shire Plc; and Takeda Pharmaceuticals. The remaining authors declare no conflict of interest.

\section{ACKNOWLEDGMENTS}

KM was supported by National Institute of Mental Health Grants R01 MH067234 and R01 MH079299. RCS was supported by National Institute of Mental Health Grants MH01741, MH52339 and MH073630 and a grant from the Brain and Behavior
Research Foundation. We are especially grateful to Weining $\mathrm{Xu}$, Senior Manager at Qiagen, for her invaluable guidance in the use of the miRNome arrays. We thank the Vantage Molecular Biology Core at Vanderbilt for their help in performing the microarray and miRNome array studies. We offer our sincere appreciation to Dr Marla Perna, who provided constructive comments on the manuscript preparation.

\section{REFERENCES}

1 Verhoeven JE, Revesz D, Wolkowitz OM, Penninx BW. Cellular aging in depression: permanent imprint or reversible process? An overview of the current evidence, mechanistic pathways, and targets for interventions. Bioessays 2014; 36 : 968-978.

2 Klengel T, Binder EB. Gene-environment interactions in major depressive disorder. Can J Psychiatry 2013; 58: 76-83.

3 Mahar I, Bambico FR, Mechawar N, Nobrega JN. Stress serotonin, and hippocampal neurogenesis in relation to depression and antidepressant effects. Neurosci Biobehav Rev 2014; 38: 173-192.

4 Yang H, Yang T, Baur JA, Perez E, Matsui T, Carmona JJ et al. Nutrient-sensitive mitochondrial NAD+ levels dictate cell survival. Cell 2007; 130: 1095-1107.

5 Galluzzi L, Kepp O, Trojel-Hansen C, Kroemer G. Mitochondrial control of cellular life, stress, and death. Circ Res 2012; 111: 1198-1207.

6 DiMauro S, Schon EA. Mitochondrial disorders in the nervous system. Annu Rev Neurosci 2008; 31: 91-123.

7 Manji H, Kato T, Di Prospero NA, Ness S, Beal MF, Krams et al. Impaired mitochondrial function in psychiatric disorders. Nat Rev Neurosci 2012; 13: 293-307.

8 Marazziti D, Baroni S, Picchetti M, Landi P, Silvestri S, Vatteroni E et al. Psychiatric disorders and mitochondrial dysfunctions. Eur Rev Med Pharmacol Sci 2012; 16: 270-275.

9 Legido A, Jethva R, Goldenthal MJ. Mitochondrial dysfunction in autism. Semin Pediatr Neurol 2013; 20: 163-175.

10 Tobe EH. Mitochondrial dysfunction, oxidative stress, and major depressive disorder. Neuropsychiatr Dis Treat 2013; 9: 567-573.

11 Gohil VM, Sheth SA, Nilsson R, Wojtovich AP, Lee JH, Perocchi F et al. Nutrientsensitized screening for drugs that shift energy metabolism from mitochondrial respiration to glycolysis. Nat Biotechnol 2010; 28: 249-255.

12 Manier DH, Shelton RC, Ellis TC, Peterson CS, Eiring A, Sulser F. Human fibroblasts as a relevant model to study signal transduction in affective disorders. $J$ Affect Disord 2000; 61: 51-58.

13 Wang L, Lockstone HE, Guest PC, Levin Y, Palotas A, Pietsch S et al. Expression profiling of fibroblasts identifies cell cycle abnormalities in schizophrenia. J Proteome Res 2010; 9: 521-527.

14 Gasso P, Mas S, Molina O, Lafuente A, Bernardo M, Parellada E. Increased susceptibility to apoptosis in cultured fibroblasts from antipsychotic-naive firstepisode schizophrenia patients. J Psychiatr Res 2014; 48: 94-101. 
15 Korade Z, Xu L, Harrison FE, Ahsen R, Hart SE, Folkes OM et al. Antioxidant supplementation ameliorates molecular deficits in Smith-Lemli-Opitz syndrome. Biol Psychiatry 2013; 75: 215-222.

$16 \mathrm{Xu} \mathrm{L}$, Korade Z, Rosado DA Jr., Mirnics K, Porter NA. Metabolism of oxysterols derived from nonenzymatic oxidation of 7-dehydrocholesterol in cells. J Lipid Res 2013; 54: 1135-1143.

17 Akin D, Manier DH, Sanders-Bush E, Shelton RC. Decreased serotonin 5-HT2A receptor-stimulated phosphoinositide signaling in fibroblasts from melancholic depressed patients. Neuropsychopharmacology 2004; 29: 2081-2087.

18 Gibson SA, Korade Z, Shelton RC. Oxidative stress and glutathione response in tissue cultures from persons with major depression. J Psychiatr Res 2012; 46: 1326-1332.

19 Kalman S, Garbett KA, Vereczkei A, Shelton RC, Korade Z, Mirnics K. Metabolic stress-induced microRNA and mRNA expression profiles of human fibroblasts. Exp Cell Res 2013; 320: 343-353.

20 Zhan Y, Yang YT, You HM, Cao D, Liu CY, Zhou CJ et al. Plasma-based proteomics reveals lipid metabolic and immunoregulatory dysregulation in post-stroke depression. Eur Psychiatry 2014; 29: 307-315.

21 Karabatsiakis A, Bock C, Salinas-Manrique J, Kolassa S, Calzia E, Dietrich DE et al. Mitochondrial respiration in peripheral blood mononuclear cells correlates with depressive subsymptoms and severity of major depression. Trans/ Psychiatry 2014; 4: e397.

22 First MB, Pincus HA. The DSM-IV Text Revision: rationale and potential impact on clinical practice. Psychiatr Serv 2002; 53: 288-292.

23 Irizarry RA, Hobbs B, Collin F, Beazer-Barclay YD, Antonellis KJ, Scherf U et al. Exploration, normalization, and summaries of high density oligonucleotide array probe level data. Biostatistics 2003; 4: 249-264.

24 Kuehn H, Liberzon A, Reich M, Mesirov JP. Using GenePattern for gene expression analysis. Curr Protoc Bioinformatics 2008; Chapter 7: Unit 7.12.

25 Subramanian A, Kuehn H, Gould J, Tamayo P, Mesirov JP. GSEA-P: a desktop application for Gene Set Enrichment Analysis. Bioinformatics 2007; 23: 3251-3253.

26 Dumortier O, Hinault C, Van Obberghen E. MicroRNAs and metabolism crosstalk in energy homeostasis. Cell Metab 2013; 18: 312-324.

27 Jiang P, Du W, Mancuso A, Wellen KE, Yang X. Reciprocal regulation of p53 and malic enzymes modulates metabolism and senescence. Nature 2013; 493: 689-693.

28 Suzuki H, Yamamoto E, Nojima M, Kai M, Yamano HO, Yoshikawa K et al. Methylation-associated silencing of microRNA-34b/c in gastric cancer and its involvement in an epigenetic field defect. Carcinogenesis 2010; 31: 2066-2073.

29 Kim S, Lee UJ, Kim MN, Lee EJ, Kim JY, Lee MY et al. MicroRNA miR-199a* regulates the MET proto-oncogene and the downstream extracellular signalregulated kinase 2 (ERK2). J Biol Chem 2008; 283: 18158-18166.

30 Shelton RC, Claiborne J, Sidoryk-Wegrzynowicz M, Reddy R, Aschner M, Lewis DA et al. Altered expression of genes involved in inflammation and apoptosis in frontal cortex in major depression. Mol Psychiatry 2011; 16: 751-762.

31 Manji HK, Drevets WC, Charney DS. The cellular neurobiology of depression. Nat Med 2001; 7: 541-547.

32 Garbett KA, Vereczkei A, Kalman S, Brown JA, Taylor WD, Faludi G et al. Coordinated messenger RNA/MicroRNA changes in fibroblasts of patients with major depression. Biol Psychiatry 2014; 77: 256-265.

33 Baillat D, Hakimi MA, Naar AM, Shilatifard A, Cooch N, Shiekhattar R. Integrator, a multiprotein mediator of small nuclear RNA processing, associates with the C-terminal repeat of RNA polymerase II. Cell 2005; 123: 265-276.

34 Wang X, Trotman LC, Koppie T, Alimonti A, Chen Z, Gao Z et al. NEDD4-1 is a proto-oncogenic ubiquitin ligase for PTEN. Cell 2007; 128: 129-139.

35 Xu K, Chen Z, Qin C, Song X. miR-7 inhibits colorectal cancer cell proliferation and induces apoptosis by targeting XRCC2. Onco Targets Ther 2014; 7: 325-332.

36 Cazanave SC, Mott JL, Elmi NA, Bronk SF, Masuoka HC, Charlton MR et al. A role for miR-296 in the regulation of lipoapoptosis by targeting PUMA. J Lipid Res 2011; 52: 1517-1525.

37 Li S, Meng H, Zhou F, Zhai L, Zhang L, Gu F et al. MicroRNA-132 is frequently down-regulated in ductal carcinoma in situ (DCIS) of breast and acts as a tumor suppressor by inhibiting cell proliferation. Pathol Res Pract 2013; 209: 179-183.

38 Hibbeln JR, Salem N Jr. Dietary polyunsaturated fatty acids and depression: when cholesterol does not satisfy. Am J Clin Nutr 1995; 62: 1-9.

39 Maes M, Christophe A, Delanghe J, Altamura C, Neels H, Meltzer HY. Lowered omega3 polyunsaturated fatty acids in serum phospholipids and cholesteryl esters of depressed patients. Psychiatry Res 1999; 85: 275-291.

40 Mamalakis G, Tornaritis M, Kafatos A. Depression and adipose essential polyunsaturated fatty acids. Prostaglandins Leukot Essent Fatty Acids 2002; 67: 311-318.

41 Su KP, Huang SY, Chiu CC, Shen WW. Omega-3 fatty acids in major depressive disorder. A preliminary double-blind, placebo-controlled trial. Eur Neuropsychopharmacol 2003; 13: 267-271.
42 Kaddurah-Daouk R, Bogdanov MB, Wikoff WR, Zhu H, Boyle SH, Churchill E et al. Pharmacometabolomic mapping of early biochemical changes induced by sertraline and placebo. Transl Psychiatry 2013; 3: e223.

43 Cottrill KA, Chan SY, Loscalzo J. Hypoxamirs and Mitochondrial Metabolism. Antioxid Redox Signal 2013; 21: 1189-1201.

44 Nallamshetty S, Chan SY, Loscalzo J. Hypoxia: a master regulator of microRNA biogenesis and activity. Free Radic Biol Med 2013; 64: 20-30.

45 Liu CR, Miao J, Zhang YL, Liu YM, Yu BG. Effects of hypothyroidism on expression of CRMP2B and ARPC5 during development of the rat frontal cortex. Int J Biol Sci 2013; 9: 209-218.

46 Kobayashi H, Fukuda M. Rab35 regulates Arf6 activity through centaurin-beta2 (ACAP2) during neurite outgrowth. J Cell Sci 2012; 125: 2235-2243.

47 Kahler AK, Djurovic S, Rimol LM, Brown AA, Athanasiu L, Jonsson EG et al. Candidate gene analysis of the human natural killer-1 carbohydrate pathway and perineuronal nets in schizophrenia: B3GAT2 is associated with disease risk and cortical surface area. Biol Psychiatry 2011; 69: 90-96.

48 Liu J, Githinji J, McLaughlin B, Wilczek K, Nolta J. Role of miRNAs in neuronal differentiation from human embryonic stem cell-derived neural stem cells. Stem Cell Rev 2012; 8: 1129-1137.

$49 \mathrm{Li} \mathrm{B}$, Sun H. MiR-26a promotes neurite outgrowth by repressing PTEN expression. Mol Med Rep 2013; 8: 676-680.

50 Agostini M, Tucci P, Steinert JR, Shalom-Feuerstein R, Rouleau M, Aberdam D et al. microRNA-34a regulates neurite outgrowth, spinal morphology, and function. Proc Natl Acad Sci USA 2011; 108: 21099-21104.

51 Khudayberdiev S, Fiore R, Schratt G. MicroRNA as modulators of neuronal responses. Commun Integr Biol 2009; 2: 411-413.

52 Numakawa T, Richards M, Adachi N, Kishi S, Kunugi H, Hashido K. MicroRNA function and neurotrophin BDNF. Neurochem Int 2011; 59: 551-558.

53 Hancock ML, Preitner N, Quan J, Flanagan JG. MicroRNA-132 is enriched in developing axons, locally regulates Rasa 1 mRNA, and promotes axon extension. J Neurosci 2014; 34: 66-78.

54 Rodriguez-Aznar E, Barrallo-Gimeno A, Nieto MA. Scratch2 prevents cell cycle reentry by repressing miR-25 in postmitotic primary neurons. J Neurosci 2009; 33: 5095-5105.

55 Aranha MM, Santos DM, Sola S, Steer CJ, Rodrigues CM. miR-34a regulates mouse neural stem cell differentiation. PLoS One 2011; 6: e21396.

56 Yadav S, Pandey A, Shukla A, Talwelkar SS, Kumar A, Pant AB et al. miR-497 and miR-302b regulate ethanol-induced neuronal cell death through $B C L 2$ protein and cyclin D2. J Biol Chem 2011; 286: 37347-37357.

57 Jovicic A, Roshan R, Moisoi N, Pradervand S, Moser R, Pillai B et al. Comprehensive expression analyses of neural cell-type-specific miRNAs identify new determinants of the specification and maintenance of neuronal phenotypes. $J$ Neurosci 2013; 33: 5127-5137.

58 Pollock A, Bian S, Zhang C, Chen Z, Sun T. Growth of the developing cerebral cortex is controlled by microRNA-7 through the p53 pathway. Cell Rep 2014; 7: 1184-1196.

59 Qi L, Hongjuan H, Ning G, Zhengbin H, Yanjiang X, Tiebo Z et al. miR-370 is stage-specifically expressed during mouse embryonic development and regulates Dnmt3a. FEBS Lett 2013; 587: 775-781.

60 Lin ST, Huang Y, Zhang L, Heng MY, Ptacek L, Fu YH. MicroRNA-23a promotes myelination in the central nervous system. Proc Natl Acad Sci USA 2013; 110: $17468-17473$.

61 Miller BH, Zeier Z, Xi L, Lanz TA, Deng S, Strathmann J et al. MicroRNA-132 dysregulation in schizophrenia has implications for both neurodevelopment and adult brain function. Proc Natl Acad Sci USA 2012; 109: 3125-3130.

62 Santarelli DM, Beveridge NJ, Tooney PA, Cairns MJ. Upregulation of dicer and microRNA expression in the dorsolateral prefrontal cortex Brodmann area 46 in schizophrenia. Biol Psychiatry 2011; 69: 180-187.

$63 \mathrm{Hu}$ YK, Wang X, Li L, Du YH, Ye HT, Li CY. MicroRNA-98 induces an Alzheimer's disease-like disturbance by targeting insulin-like growth factor 1. Neurosci Bull 2013; 29: 745-751.

64 Lai CY, Yu SL, Hsieh MH, Chen CH, Chen HY, Wen CC et al. MicroRNA expression aberration as potential peripheral blood biomarkers for schizophrenia. PLoS One 2011; 6: e21635.

65 Mor E, Kano S, Colantuoni C, Sawa A, Navon R, Shomron N. MicroRNA-382 expression is elevated in the olfactory neuroepithelium of schizophrenia patients. Neurobiol Dis 2013; 55: 1-10.

66 Kumar P, Dezso Z, MacKenzie C, Oestreicher J, Agoulnik S, Byrne et al. Circulating miRNA biomarkers for Alzheimer's disease. PLoS One 2013; 8: e69807.

67 Gaughwin PM, Ciesla M, Lahiri N, Tabrizi SJ, Brundin P, Bjorkqvist M. Hsa-miR-34b is a plasma-stable microRNA that is elevated in pre-manifest Huntington's disease. Hum Mol Genet 2011; 20: 2225-2237.

68 Minones-Moyano E, Porta S, Escaramis G, Rabionet R, Iraola S, Kagerbauer B et al. MicroRNA profiling of Parkinson's disease brains identifies early downregulation 
of miR-34b/c which modulate mitochondrial function. Hum Mol Genet 2011; 20 3067-3078.

69 Zhang R, Zhang Q, Niu J, Lu K, Xie B, Cui D et al. Screening of microRNAs associated with Alzheimer's disease using oxidative stress cell model and different strains of senescence accelerated mice. J Neurol Sci 2014; 338: 57-64.

70 Truettner JS, Katyshev V, Esen-Bilgin N, Dietrich WD, Dore-Duffy P. Hypoxia alters MicroRNA expression in rat cortical pericytes. Microrna 2013; 2: 32-45.

71 Zucchi FC, Yao Y, Ward ID, Inytskyy Y, Olson DM, Benzies K et al. Maternal stress induces epigenetic signatures of psychiatric and neurological diseases in the offspring. PLoS One 2013; 8: e56967.

72 Robson JE, Eaton SA, Underhill P, Williams D, Peters J. MicroRNAs 296 and 298 are imprinted and part of the GNAS/Gnas cluster and miR-296 targets IKBKE and Tmed9. RNA 2012; 18: 135-144.

73 Koido K, Eller T, Kingo K, Koks S, Traks T, Shlik J et al. Interleukin 10 family gene polymorphisms are not associated with major depressive disorder and panic disorder phenotypes. J Psychiatr Res 2010; 44: 275-277.

74 Davalos A, Goedeke L, Smibert P, Ramirez CM, Warrier NP, Andreo U et al. miR-33a/b contribute to the regulation of fatty acid metabolism and insulin signaling. Proc Natl Acad Sci USA 2011; 108: 9232-9237.

75 Wang $\mathrm{H}$, Zheng $\mathrm{Y}$, Wang G, Li H. Identification of microRNA and bioinformatics target gene analysis in beef cattle intramuscular fat and subcutaneous fat. $\mathrm{Mol}$ Biosyst 2013; 9: 2154-2162.

76 Gao W, He HW, Wang ZM, Zhao H, Lian XQ, Wang YS et al. Plasma levels of lipometabolism-related miR-122 and miR-370 are increased in patients with hyperlipidemia and associated with coronary artery disease. Lipids Health Dis 2012; 11: 55.

77 Zhang Y, Cheng X, Lu Z, Wang J, Chen H, Fan W et al. Upregulation of miR-15b in NAFLD models and in the serum of patients with fatty liver disease. Diabetes Res Clin Pract 2013; 99: 327-334.

78 el Azzouzi H, Leptidis S, Dirkx E, Hoeks J, van Bree B, Brand K et al. The hypoxiainducible microRNA cluster miR-199a approximately 214 targets myocardia PPARdelta and impairs mitochondrial fatty acid oxidation. Cell Metab 2013; 18: 341-354.

79 Guo ST, Jiang CC, Wang GP, Li YP, Wang CY, Guo XY et al. MicroRNA-497 targets insulin-like growth factor 1 receptor and has a tumour suppressive role in human colorectal cancer. Oncogene 2013; 32: 1910-1920.

80 Tekirdag KA, Korkmaz G, Ozturk DG, Agami R, Gozuacik D. MIR181A regulates starvation- and rapamycin-induced autophagy through targeting of ATG5. Autophagy 2013; 9: 374-385.

81 Wei Z, Cui L, Mei Z, Liu M, Zhang D. miR-181a mediates metabolic shift in colon cancer cells via the PTEN/AKT pathway. FEBS Lett 2014; 588: 1773-1779.

82 Meerson A, Traurig M, Ossowski V, Fleming JM, Mullins M, Baier LJ. Human adipose microRNA-221 is upregulated in obesity and affects fat metabolism downstream of leptin and TNF-alpha. Diabetologia 2013; 56: 1971-1979.

83 Hoffmeister A, Tuennemann J, Sommerer I, Mossner J, Rittger A, Schleinitz D et al. Genetic and biochemical evidence for a functional role of BACE1 in the regulation of insulin mRNA expression. Obesity (Silver Spring) 2013; 21 E626-E633.

84 Collares CV, Evangelista AF, Xavier DJ, Rassi DM, Arns T, Foss-Freitas MC et al. Identifying common and specific microRNAs expressed in peripheral blood mononuclear cell of type 1, type 2, and gestational diabetes mellitus patients. BMC Res Notes 2013; 6: 491.

85 Zehavi L, Avraham R, Barzilai A, Bar-llan D, Navon R, Sidi Y et al. Silencing of a large microRNA cluster on human chromosome $14 q 32$ in melanoma: biological effects of mir-376a and mir-376c on insulin growth factor 1 receptor. Mol Cancer 2012; 11: 44.

86 Setyowati Karolina D, Sepramaniam S, Tan HZ, Armugam A, Jeyaseelan K. miR-25 and miR-92a regulate insulin I biosynthesis in rats. RNA Biol 2013; 10: 1365-1378.

87 Latreille M, Hausser J, Stutzer I, Zhang Q, Hastoy B, Gargani S et al. MicroRNA-7a regulates pancreatic beta cell function. J Clin Invest 2014; 124: 2722-2735.

$88 \mathrm{Kim}$ HR, Roe JS, Lee JE, Cho EJ, Youn HD. p53 regulates glucose metabolism by miR-34a. Biochem Biophys Res Commun 2013; 437: 225-231.

89 Karbiener M, Pisani DF, Frontini A, Oberreiter LM, Lang E, Vegiopoulos A et al. MicroRNA-26 family is required for human adipogenesis and drives characteristics of brown adipocytes. Stem Cells 2014; 32: 1578-1590.

90 Chen B, Liu Y, Jin X, Lu W, Liu J, Xia Z et al. MicroRNA-26a regulates glucose metabolism by direct targeting PDHX in colorectal cancer cells. BMC Cancer 2014; 14: 443

91 Gil-Zamorano J, Martin R, Daimiel L, Richardson K, Giordano E, Nicod N et al. Docosahexaenoic acid modulates the enterocyte Caco-2 cell expression of microRNAs involved in lipid metabolism. J Nutr 2014; 144: 575-585.

92 Sangiao-Alvarellos S, Pena-Bello L, Manfredi-Lozano M, Tena-Sempere M, Cordido F. Perturbation of hypothalamic microRNA expression patterns in male rats after metabolic distress: impact of obesity and conditions of negative energy balance. Endocrinology 2014; 155: 1838-1850.
93 Fu T, Seok S, Choi S, Huang Z, Suino-Powell K, Xu HE et al. MiR-34a inhibits beige and brown fat formation in obesity in part by suppressing adipocyte FGF21 signaling and SIRT1 function. Mol Cell Biol 2014; 34: 4130-4142.

94 Gao P, Tchernyshyov I, Chang TC, Lee YS, Kita K, Ochi T et al. c-Myc suppression of miR-23a/b enhances mitochondrial glutaminase expression and glutamine metabolism. Nature 2009; 458: 762-765.

95 Hoffman Y, Bublik DR, Pilpel Y, Oren M. miR-661 downregulates both Mdm2 and Mdm4 to activate p53. Cell Death Differ 2013; 21: 302-309.

96 Wang J, He Q, Han C, Gu H, Jin L, Li Q et al. p53-facilitated miR-199a-3p regulates somatic cell reprogramming. Stem Cells 2012; 30: 1405-1413.

97 Barbagallo D, Piro S, Condorelli AG, Mascali LG, Urbano F, Parrinello N et al. miR-296-3p, miR-298-5p and their downstream networks are causally involved in the higher resistance of mammalian pancreatic alpha cells to cytokine-induced apoptosis as compared to beta cells. BMC Genomics 2013; 14: 62.

98 Rokavec M, Li H, Jiang L, Hermeking $\mathrm{H}$. The p53/miR-34 axis in development and disease. J Mol Cell Biol 2014; 6: 214-230.

99 Fang L, Zhang HB, Li H, Fu Y, Yang GS. miR-548c-5p inhibits proliferation and migration and promotes apoptosis in CD90(+) HepG2 cells. Radiol Oncol 2012; 46: 233-241.

100 Seok JK, Lee SH, Kim MJ, Lee YM. MicroRNA-382 induced by HIF-1alpha is an angiogenic miR targeting the tumor suppressor phosphatase and tensin homolog. Nucleic Acids Res 2014; 42: 8062-8072.

101 Kwon JE, Kim BY, Kwak SY, Bae IH, Han YH. lonizing radiation-inducible microRNA miR-193a-3p induces apoptosis by directly targeting Mcl-1. Apoptosis 2013; 18: 896-909.

102 Tian S, Huang S, Wu S, Guo W, Li J, He X. MicroRNA-1285 inhibits the expression of p53 by directly targeting its 3' untranslated region. Biochem Biophys Res Commun 2010; 396: 435-439.

103 Wu W, Yang J, Feng X, Wang H, Ye S, Yang $\mathrm{P}$ et al. MicroRNA-32 (miR-32) regulates phosphatase and tensin homologue (PTEN) expression and promotes growth, migration, and invasion in colorectal carcinoma cells. Mol Cancer 2013 12: 30 .

104 Shi L, Jackstadt R, Siemens H, Li H, Kirchner T, Hermeking H. p53-induced miR-15a/16-1 and AP4 form a double-negative feedback loop to regulate epithelial-mesenchymal transition and metastasis in colorectal cancer. Cancer Res 2013; 74: 532-542.

105 Song B, Wang Y, Kudo K, Gavin EJ, Xi Y, Ju J. miR-192 regulates dihydrofolate reductase and cellular proliferation through the p53-microRNA circuit. Clin Cancer Res 2008; 14: 8080-8086.

106 Wang F, Yu J, Yang GH, Wang XS, Zhang JW. Regulation of erythroid differentiation by miR-376a and its targets. Cell Res 2011; 21: 1196-1209.

107 Gentilin E, Tagliati F, Filieri C, Mole D, Minoia M, Rosaria Ambrosio et al. miR-26a plays an important role in cell cycle regulation in ACTH-secreting pituitary adenomas by modulating protein kinase Cdelta. Endocrinology 2013; 154: 1690-1700.

108 Boominathan L. The tumor suppressors p53, p63, and p73 are regulators of microRNA processing complex. PLoS One 2010; 5: e10615.

109 Khan S, Brougham CL, Ryan J, Sahrudin A, O'Neill G, Wall D et al. miR-379 regulates cyclin B1 expression and is decreased in breast cancer. PLoS One 2013; 8: e68753.

110 Zhong Z, Dong Z, Yang L, Chen X, Gong Z. Inhibition of proliferation of human lung cancer cells by green tea catechins is mediated by upregulation of let-7. Exp Ther Med 2012; 4: 267-272.

111 Zhang S, Zhang C, Li Y, Wang P, Yue Z, Xie S. miR-98 regulates cisplatin-induced A549 cell death by inhibiting TP53 pathway. Biomed Pharmacother 2011; 65 436-442.

112 Tian Y, Zhang YZ, Chen W. MicroRNA-199a-3p and microRNA-34a regulate apoptosis in human osteosarcoma cells. Biosci Rep 2014; 34: e00132.

113 Cao X, Liu D, Yan X, Zhang Y, Yuan L, Zhang T et al. Stat3 inhibits WTX expression through up-regulation of microRNA-370 in Wilms tumor. FEBS Lett 2013; 587: 639-644.

114 Rahman M, Lovat F, Romano G, Calore F, Acunzo M, Bell EH et al. miR-15b/16-2 regulates factors that promote $\mathrm{p} 53$ phosphorylation and augments the DNA damage response following radiation in the lung. J Biol Chem 2014; 289: 26406-26416

115 Ruan W, Xu JM, Li SB, Yuan LQ, Dai RP. Effects of down-regulation of microRNA-23a on TNF-alpha-induced endothelial cell apoptosis through caspase-dependent pathways. Cardiovasc Res 2011; 93: 623-632.

116 Cirera-Salinas D, Pauta M, Allen RM, Salerno AG, Ramirez CM, Chamorro-Jorganes A et al. Mir-33 regulates cell proliferation and cell cycle progression. Cell Cycle 2012; 11: 922-933.

117 Rippo MR, Olivieri F, Monsurro V, Prattichizzo F, Albertini MC, Procopio AD. MitomiRs in human inflamm-aging: a hypothesis involving miR-181a, miR-34a and miR-146a. Exp Gerontol 2014; 56: 154-163. 
118 Lee YM, Lee JY, Ho CC, Hong QS, Yu SL, Tzeng CR et al. miRNA-34b as a tumor suppressor in estrogen-dependent growth of breast cancer cells. Breast Cancer Res 2011; 13: R116.

119 Reddy SD, Pakala SB, Ohshiro K, Rayala SK, Kumar R. MicroRNA-661 a c/EBPalpha target, inhibits metastatic tumor antigen 1 and regulates its functions. Cancer Res 2009; 69: 5639-5642.

120 Choudhury Y, Tay FC, Lam DH, Sandanaraj E, Tang C, Ang BT et al. Attenuated adenosine-to-inosine editing of microRNA-376a* promotes invasiveness of glioblastoma cells. J Clin Invest 2012; 122: 4059-4076.

121 Huang SD, Yuan Y, Zhuang CW, Li BL, Gong DJ, Wang SG et al. MicroRNA-98 and microRNA-214 post-transcriptionally regulate enhancer of zeste homolog 2 and inhibit migration and invasion in human esophageal squamous cell carcinoma. Mol Cancer 2012; 11: 51.

122 Vaira V, Faversani A, Dohi T, Montorsi M, Augello C, Gatti S et al. miR-296 regulation of a cell polarity-cell plasticity module controls tumor progression. Oncogene 2012; 31: 27-38.

123 Tian Q, Liang L, Ding J, Zha R, Shi H, Wang Q et al. MicroRNA-550a acts as a pro-metastatic gene and directly targets cytoplasmic polyadenylation element-binding protein 4 in hepatocellular carcinoma. PLoS One 2012; 7: e48958.

124 Jiang DS, Wang YW, Jiang J, Li SM, Liang SZ, Fang HY. MicroRNA-26a involved in Toll-like receptor 9mediated lung cancer growth and migration. Int J Mol Med 2014; 34: 307-312.

125 Liu DZ, Ander BP, Tian Y, Stamova B, Jickling GC, Davis RR et al. Integrated analysis of mRNA and microRNA expression in mature neurons, neural progenitor cells and neuroblastoma cells. Gene 2012; 495: 120-127.

126 Menges CW, Kadariya Y, Altomare D, Talarchek J, Neumann-Domer E, Wu Y et al. Tumor suppressor alterations cooperate to drive aggressive mesotheliomas with enriched cancer stem cells via a p53-miR-34a-c-Met axis. Cancer Res 2014; 74: 1261-1271.
127 Wang LG, Ni Y, Su BH, Mu XR, Shen HC, Du JJ. MicroRNA-34b functions as a tumor suppressor and acts as a nodal point in the feedback loop with Met. Int $J$ Oncol 2013; 42: 957-962.

128 Niu CS, Yang Y, Cheng CD. MiR-134 regulates the proliferation and invasion of glioblastoma cells by reducing Nanog expression. Int J Oncol 2013; 42: 1533-1540.

129 You J, Li Y, Fang N, Liu B, Zu L, Chang R et al. MiR-132 suppresses the migration and invasion of lung cancer cells via targeting the EMT regulator ZEB2. PLoS One 2014; 9: e91827.

130 Feng S, Pan W, Jin Y, Zheng J. MiR-25 promotes ovarian cancer proliferation and motility by targeting LATS2. Tumour Biol 2014; 35: 12339-12344.

131 Wang M, Ren D, Guo W, Wang Z, Huang S, Du H et al. Loss of miR-100 enhances migration, invasion, epithelial-mesenchymal transition and stemness properties in prostate cancer cells through targeting Argonaute 2. Int J Oncol 2014; 45: 362-372.

132 Li J, Liu X, Qin S, Guan Y, Liu Y, Cheng Y et al. MicroRNA expression profile and functional analysis reveal that miR-382 is a critical novel gene of alcohol addiction. EMBO Mol Med 2013; 5: 1402-1414.

133 Ekdahl Y, Farahani HS, Behm M, Lagergren J, Ohman M. A-to-I editing of microRNAs in the mammalian brain increases during development. Genome Res 2012; 22: 1477-1487.

134 Gaughwin P, Ciesla M, Yang H, Lim B, Brundin P. Stage-specific modulation of cortical neuronal development by Mmu-miR-134. Cereb Cortex 2011; 21: 1857-1869.

(c) (i) $\Theta$ This work is licensed under a Creative Commons AttributionNonCommercial-NoDerivs 4.0 International License. The images or other third party material in this article are included in the article's Creative Commons license, unless indicated otherwise in the credit line; if the material is not included under the Creative Commons license, users will need to obtain permission from the license holder to reproduce the material. To view a copy of this license, visit http:// creativecommons.org/licenses/by-nc-nd/4.0/

Supplementary Information accompanies the paper on the Translational Psychiatry website (http://www.nature.com/tp) 
$\int \sqrt{ }$ 


\section{REVIEW}

\section{HUMAN DERMAL FIBROBLASTS IN PSYCHIATRY RESEARCH}

\author{
S. KÁLMÁN, ${ }^{a}$ K. A. GARBETT, ${ }^{b}$ Z. JANKA ${ }^{a}$ AND \\ K. MIRNICS ${ }^{a, b *}$ \\ a Department of Psychiatry, University of Szeged, 57 Kálvária \\ Sgt, Szeged 6725, Hungary \\ ${ }^{\mathrm{b}}$ Department of Psychiatry, Vanderbilt University, 8128 MRB III, \\ 465 21st Avenue, Nashville, TN 37232, USA
}

\begin{abstract}
In order to decipher the disease etiology, progression and treatment of multifactorial human brain diseases we utilize a host of different experimental models. Recently, patient-derived human dermal fibroblast (HDF) cultures have re-emerged as promising in vitro functional system for examining various cellular, molecular, metabolic and (patho)physiological states and traits of psychiatric disorders. HDF studies serve as a powerful complement to postmortem and animal studies, and often appear to be informative about the altered homeostasis in neural tissue. Studies of HDFs from patients with schizophrenia (SZ), depression, bipolar disorder (BD), autism, attention deficit and hyperactivity disorder and other psychiatric disorders have significantly advanced our understanding of these devastating diseases. These reports unequivocally prove that signal transduction, redox homeostasis, circadian rhythms and gene*environment $\left(G^{*} E\right)$ interactions are all amenable for assessment by the HDF model. Furthermore, the reported findings suggest that this underutilized patient biomaterial, combined with modern molecular biology techniques, may have both diagnostic and prognostic value, including prediction of response to therapeutic agents. (C) 2016 IBRO. Published by Elsevier Ltd. All rights reserved.
\end{abstract}

Key words: dermal fibroblasts, schizophrenia, major depression, bipolar disorder, in vitro model, gene*environment interaction.

\begin{tabular}{lc}
\multicolumn{2}{c}{ Contents } \\
The need for multiple model systems & 105 \\
Patient biomaterial models, advantages and limitations & 106 \\
Peripheral leukocytes & 107 \\
Immortalized lymphocytes & 107 \\
Olfactory epithelium & 107 \\
Induced pluripotent stem cells (iPSC) & 107 \\
iNCs & 108 \\
HDFs & 108 \\
Utility of fibroblasts in psychiatric research & 109 \\
Understanding pathophysiological processes using HDFs & 110 \\
Signaling pathway studies & 110 \\
Circadian rhythm studies & 113 \\
Studies of stress and adaptation & 113 \\
Redox state studies & 114 \\
Age-related studies & 114 \\
Data-driven studies & 114 \\
Studies of pharmacological agents & 115 \\
Similarities between HDF and CNS cells & 115 \\
Conclusions & 116 \\
Acknowledgements & 116 \\
References & 116
\end{tabular}

\section{THE NEED FOR MULTIPLE MODEL SYSTEMS}

Models simplify complex processes, isolate critical pathophysiological components and allow experimentation. In order to study a system, the model has to be able to test cause-and-effect relationships. Yet, compromise is unavoidable: we select a model for its desired features, and the presumably "less relevant" variables are sacrificed for the sake of simplicity. Obviously, the choice of the model should always be guided by the tested hypothesis, as the combination of various models should result in conclusive data and gain of new knowledge.

Considering that the brain pathophysiology of psychiatric disorders can be only analyzed in humans by non-invasive methods, animal models are the preferred choice to examine behavioral disturbances and associated structural and functional brain alterations that arise from genetic manipulations (Schmidt and Mirnics, 2012; Schmidt et al., 2014; Brown et al., 2015). Although these experiments are essential, psychiatric disorders are uniquely human conditions, and cannot be interpreted in the disease context without understanding the events in the diseased tissue (Nestler and Hyman, 2010). 
Brain biopsies from psychiatric patients are very rarely justified, and even when brain biopsy tissue is obtained, keeping mature neurons alive is extremely challenging (Hayashi-Takagi et al., 2014). As a result, postmortem studies are the preferred choice to study disease-related transcriptome, proteome and metabolome changes. However, these tissue samples have limited utility for the most critical functional assays, such as evaluating gene*environment $\left(G^{*} E\right)$ interactions, stress resilience or drug effects (Horváth et al., 2011; Horváth and Mirnics, 2015). Additionally, as this is a sample of opportunity, the interpretation of findings is greatly complicated by confounds such as individual lifestyle differences, comorbidity, drug abuse, medication use, postmortem interval, hormonal status, cause of death and many other factors (Mirnics et al., 2006; Schmitt et al., 2008). Furthermore, perhaps the greatest intrinsic limitation of postmortem tissue is its limited utility in establishing diagnosis and aiding therapeutic approaches.

\section{PATIENT BIOMATERIAL MODELS, ADVANTAGES AND LIMITATIONS}

Psychiatric disorders are multifactorial and multidimensional diseases: the pathology and pathophysiology encompass biological events that span genetic, molecular, cellular, neurochemical, systemic, psychological and social domains (Schmitt et al., 2014). As a result, all human-based in vitro experimental systems have limitations, but also offer significant advantages: they allow mechanistic experimentation on patient biomaterial, and this is especially important as none of the in vivo rodent models of psychiatric disorders can fully recapitulate human genetic diversity and overall disease phenotype (Sarnyai et al., 2011; Kaiser and Feng, 2015).

There is a strong need for patient-derived biospecimens that can be propagated, preferably without altering the genetic makeup of the patients. Importantly, the pathophysiological mechanisms associated with psychiatric disorders such as schizophrenia (SZ) can be investigated in cell cultures as (1) they show high heritability, suggesting a significant genetic contribution to the disease, which would be preserved in an in vitro model (Brennand et al., 2012); (2) psychiatric disorders appear to be a disease of the whole body (and not only the brain), therefore many of the disease-associated immune, endocrine and metabolic dysregulations should be detectable in peripheral cells as well (Penninx et al., 2013; Shivakumar et al., 2014); (3) the molecular anomalies which contribute to the neural dysfunction (e.g. altered oxidative and energy homeostasis, mitochondrial changes, metabolic disruptions, signal transduction disturbances) are also present in patient-derived peripheral tissues.

Investigators have been testing meaningful patientderived functional experimental models for decades. To date, the most commonly used patient-derived models include (1) peripheral blood leukocytes; (2) immortalized peripheral lymphocytes; (3) olfactory epithelium, (4) induced pluripotent stem cells (iPCSs), (5) induced neural progenitor cells (iNPCs) and induced neuronal

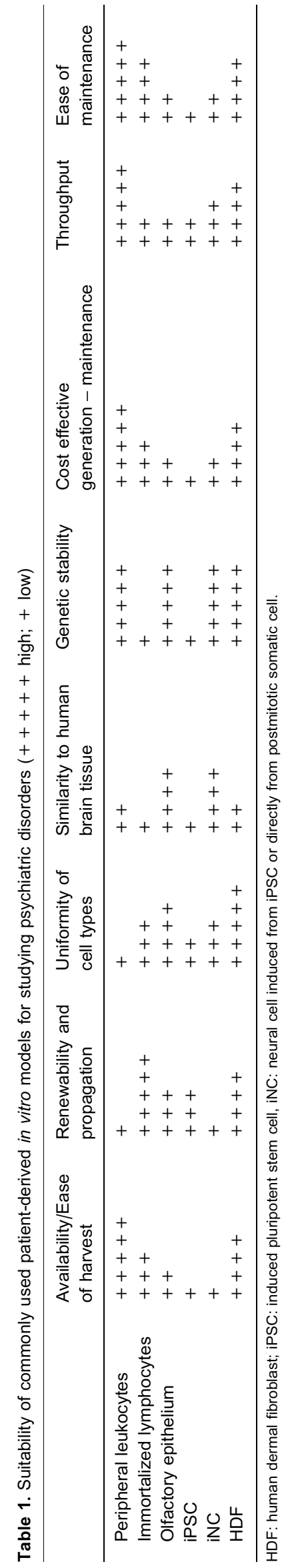


cells (iNCs) and (6) human dermal fibroblasts (HDFs) (Hayashi-Takagi et al., 2014). Each of these cellular models have advantages and shortcomings, described below and summarized in Table 1.

In addition to the inherent differences between all cellular models, they all share a common limitation: the cells are maintained in an artificial environment. The biology of all cells (including, but not limited to proliferation, migration, and function) is highly dependent on the microenvironment. Unfortunately, we have limited understanding of in vivo spatiotemporal relationships and microenvironments (Lu et al., 2011; Tatullo et al., 2015). We cannot recreate them in our in vitro culturing environments, and the physiological niche of the dissociated cells is certainly far from ideal. For example, we know that self-maintenance and renewal of cell cultures is highly dependent on the extracellular matrix $(E C M)$ remodeling in a cell-type-specific fashion (Przybyla et al., 2013): it appears that some cells grown in vitro are capable of modifying their microenvironment by producing ECM-proteins, glycopeptides and proteases (e.g. cultured astroglia or HDFs) (Biran et al., 1999; Fullár et al., 2015), while other cell types (e.g. pluripotent stem cells and neurons) require support in niche-modulation from feeder cells such as mouse embryonic fibroblasts or astroglia (Moore et al., 2009; Przybyla et al., 2013).

\section{Peripheral leukocytes}

Peripheral leukocytes, and particularly peripheral bloodderived mononuclear cells have a long history of use in psychiatric research, assuming a bidirectional communication between the immune and nervous systems (Naydenov et al., 2007; Khandaker and Dantzer, 2015). It has been argued that peripheral leukocytes and brain cells share considerable similarities in their proteome, signaling signature, and hormone and drug response (Gladkevich et al., 2004; Iga et al., 2008). Several studies revealed peripheral leukocyte number and functional alterations in psychiatric diseases, establishing it as a useful ex vivo model (reviewed in Gladkevich et al., 2004; Iga et al., 2008). The main advantages of the peripheral leukocyte/mononuclear cell model are that (1) they can be easily and repeatedly harvested during the course of the illness, and (2) they are reactive to external and internal stimuli, allowing monitoring of the disease state. In contrast, the main drawbacks of the peripheral blood cell models are the considerable individual variability and sensitivity to factors that are independent of the course of the neuropsychiatric disorders (e.g. lifestyle, hormonal status, circadian rhythm, exposure to pathogens) (Gladkevich et al., 2004; Druzd et al., 2014). In addition, the number and function of circulating leukocytes are affected by the psychopharmacological treatment (Munzer et al., 2013; Tourjman et al., 2013). All these factors are a considerable source of "biological noise" that results in a considerable variability among subjects, often preventing discovery of small effect sizes or discerning clear causality (Krieger et al., 1998). However, peripheral blood cells are still quite useful for performing one-generation-long ex vivo functional assays (Naydenov et al., 2007).

\section{Immortalized lymphocytes}

In contrast to natively harvested, unmodified leukocytes, immortalized lymphocytes have self-renewal capacity and represent a virtually unlimited source of experimental material. However, immortalization (which introduces foreign genetic material into the cells) is a time- and resource-consuming process which might erase the majority of epigenetic modifications, and alter the DNA makeup of the cell lines to the degree that the responses might not properly mimic the cellular response found in unmodified cells (Fridman and Tainsky, 2008). In addition, immortalized cell lines exhibit significant karyotypic instability and disturbed DNA damage response (Toouli et al., 2002). Hence, these cells are more appropriate for addressing narrow focused questions in the context of the donor's genetic background (e.g. the brain-derived neurotrophic factor (BDNF)-induced serotonin receptor changes) (Mössner et al., 2000), but less practical for the investigation of the broad spectrum of cellular functions (e.g. transcriptome and proteome signatures).

\section{Olfactory epithelium}

Olfactory epithelium-derived cell cultures are promising models in neuropsychiatric research (Borgmann-Winter et al., 2015). Biopsies or exfoliated tissue samples yield a mixture of multipotent stem cells, mature and developing neurons and glia (Huard et al., 1998). Consequently, ex vivo and in vitro experiments (reviewed by Borgmann-Winter et al., 2015) can be performed on cells which closely reflect the donor's neurobiology without compromising the self-renewal capacity. Unfortunately, these cell cultures remain quite diverse even after culturing (Huard et al., 1998), and represent a mixture of different cell types at various stages of differentiation, which can change based on culturing conditions (Féron et al., 2013), presented co-factors (Greiner et al., 2011), number of passages and site of the biopsy (BorgmannWinter et al., 2015). During culturing some cells maintain proliferative capacity, others progress to replicative senescence, while the highly differentiated cells respond to odorants in the primary cultures (Gomez et al., 2008). Thus, these experiments require standardized methodology, careful identification of the examined cells via molecular phenotyping, and conservative interpretation of findings. In summary, olfactory epithelium cell cultures present a unique, very valuable experimental system, with drawbacks related to the complexity of biomaterial harvest and subsequent culturing challenges.

\section{Induced pluripotent stem cells (iPSC)}

In the last decade iPSCs emerged as an exciting, disease-relevant experimental model (Tang et al., 2015). iPSCs, re-programed from somatic cells can differentiate into virtually any adult cell type, resulting in an unlimited source of patient-specific biomaterial (Takahashi et al., 2007). The technology is rapidly developing, and several protocols exist for reprogramming and differentiation with varying efficacy and results 
(Singh et al., 2015). Disease- and patient-specific iPSC lines have been created for several disorders, including Parkinson's disease (Park et al., 2008 and Soldner et al., 2011), amyotrophic lateral sclerosis (Dimos et al., 2008), spinal muscular atrophy (Ebert et al., 2009), Alzheimer's disease (Israel et al., 2012), Huntington's disease (Park et al., 2008), and SZ (Brennand et al., 2012). Still, it is worth noting that during reprogramming, unforeseen DNA mutations may occur and significant part of the epigenome becomes rearranged (Martins-Taylor and $\mathrm{Xu}, 2012$ ). This (epi)genetic instability of iPSCs could result in great heterogeneity within cultures, often necessitating performing the experiments on multiple cell lines from the same individuals (Brennand et al., 2014). Additionally, creating and maintaining iPSCs is a laborintensive, and a time- and resource-consuming endeavor.

\section{iNCs}

Currently, neural cells can be derived from somatic cells via iPSC differentiation (Hu et al., 2010) or via lineage conversion (direct transdifferentiation) (Pang et al., 2011). The reprogramming and differentiation of iPSCs into neural progenitor cells (NPCs) and mature neurons is achieved by forced expression or exogenous addition of growth factors and small molecules (reviewed by Kim et al., 2014). The obtained cells are thought to undergo the neuronal developmental pathways observed in the brain, and can be easily cultured and stored (Hu et al., 2010; Song et al., 2013). Technical challenges associated with $\mathrm{iPSC}$ research (i.e. generation time and resource demands, (epi)genetic changes, population heterogeneity and oncogenesis) motivated the development of alternative iNC generation methods, i.e. cell lineage conversion (Pang et al., 2011). Remarkably, the expression of only two microRNAs or a single transcription factor might be sufficient to induce neural cell fate in human postmitotic somatic cells and produce functional neurons (Yoo et al., 2011; Chanda et al., 2014).

Both iPSC-derived and transdifferentiated iNCs form functional synapses and resemble the receptor expression and electrophysiologic profile of neurons in the brain (Belinsky et al., 2014; Chanda et al., 2014). Moreover, iNCs with different neurotransmitter- and region-specific identity can be generated, such as GABAergic cortical interneurons (Liu et al., 2013), glutamatergic pyramidal neurons, dopaminergic midbrain neurons, or spinal motor neurons (Zeng et al., 2010). However, the iNC models need comprehensive validation by genetic, electrophysiologic and pharmacologic approaches due to the following reasons: (1) de novo copy number variations (CNVs) are likely to happen through the somatic cell - iPSC - iNC transitions (Corrales et al., 2012); (2) the maturation of neurons requires 6-12 weeks. During this time window there are virtually thousands of gene expression changes (Fathi et al., 2011; Dage et al., 2014) and the extended culture period often results in heterogeneous, asynchronic cell populations consisting of progenitors, glial cells, mature and immature neurons (Belinsky et al., 2014). (3) iPSCs "reset" in their epigenetics, telomere processing and mitochondrial organization (Rohani et al., 2014) while transdifferentiated neurons retain the donor's agerelated signature (Mertens et al., 2015).

\section{HDFs}

Notably, the HDF model excels in many of the important experimental considerations listed above: (1) they are relatively easy to obtain from ambulatory skin biopsies (Vangipuram et al., 2013, Wang et al., 2004), (2) their maintenance does not require significant equipment, chemical or human resources; (3) they can be easily propagated for long periods of time without genetic manipulation or external growth factors and stored in liquid nitrogen for years (Auburger et al., 2012;); and (4) HDF cultures consist of mitotic and post-mitotic, uniform fibroblast cells (Bayreuther et al., 1991) and retain genetic stability for 15-20 passages (Hänzelmann et al., 2015), making a single biopsy sufficient for hundreds of experiments. All these considerations make HDF cultures an appealing, cost-effective experimental model.

There are several drawbacks that should be considered when using the HDF experimental model. First, in vivo and in vitro cellular aging-related changes in HDFs must be considered, as several experiments examined the effects of donor's age on cell viability and senescence based on multiple parameters (e.g. life span, replicative life span, growth rate, replicative potential, proliferation rate). These publications reveal that mRNA, miRNA and protein levels (Waldera-Lupa et al., 2014), DNA methylation (Koch et al., 2011), intracellular calcium levels, mitochondrial function and cell size (Peterson and Goldman, 1986) are all influenced by the age of the HDF donor. These findings necessitate careful case-control age matching and/or use of senescence markers. Furthermore, to avoid consequences of in vitro cellular aging, matching of HDF passage is equally important, as multiple studies reveal that the mitotic rate (Maier et al., 2007), telomere length, DNA methylation (Hänzelmann et al., 2015), proteome and protein phosphorylation (Sprenger et al., 2010) change after the 15th passage in HDF cultures and the cells enter replicative senescence between passage 21 and 40 (Hänzelmann et al., 2015). Additionally, it is also noteworthy that using early HDF passages might be also less than ideal: during the first 3 divisions HDF cultures are not yet homogeneous (Auburger et al., 2012) and are undergoing adaptation to the culturing conditions that result in both transcriptome and epigenome remodeling (Antequera et al., 1990; Nestor et al., 2015). Similarly, Akin et al. (2004) suggest that patients' dietary, hormonal and medication effects disappear after the 5th HDF passage. Other, consistent reports suggest that body mass index (BMI), smoking and medication use of the biopsy donor do not influence the metabolome of HDF cultures past several passages (Fournier et al., 2014). Therefore, it appears that case-control HDF experiments produce the most consistent and reproducible findings when performed on HDFs between passages 5 and 15 .

In addition, HDFs are as variable as the human beings they originate from. HDF signal transduction (Gaspar et al., 2014), mitochondrial function, circadian period length (Brown et al., 2005) and cell size (Huang et al., 2005) 
Table 2. The advantages and limitations of human dermal fibroblast (HDF) cultures as in vitro model of psychiatric disorders (based on Auburger et al. (2012) and Connolly (1998))

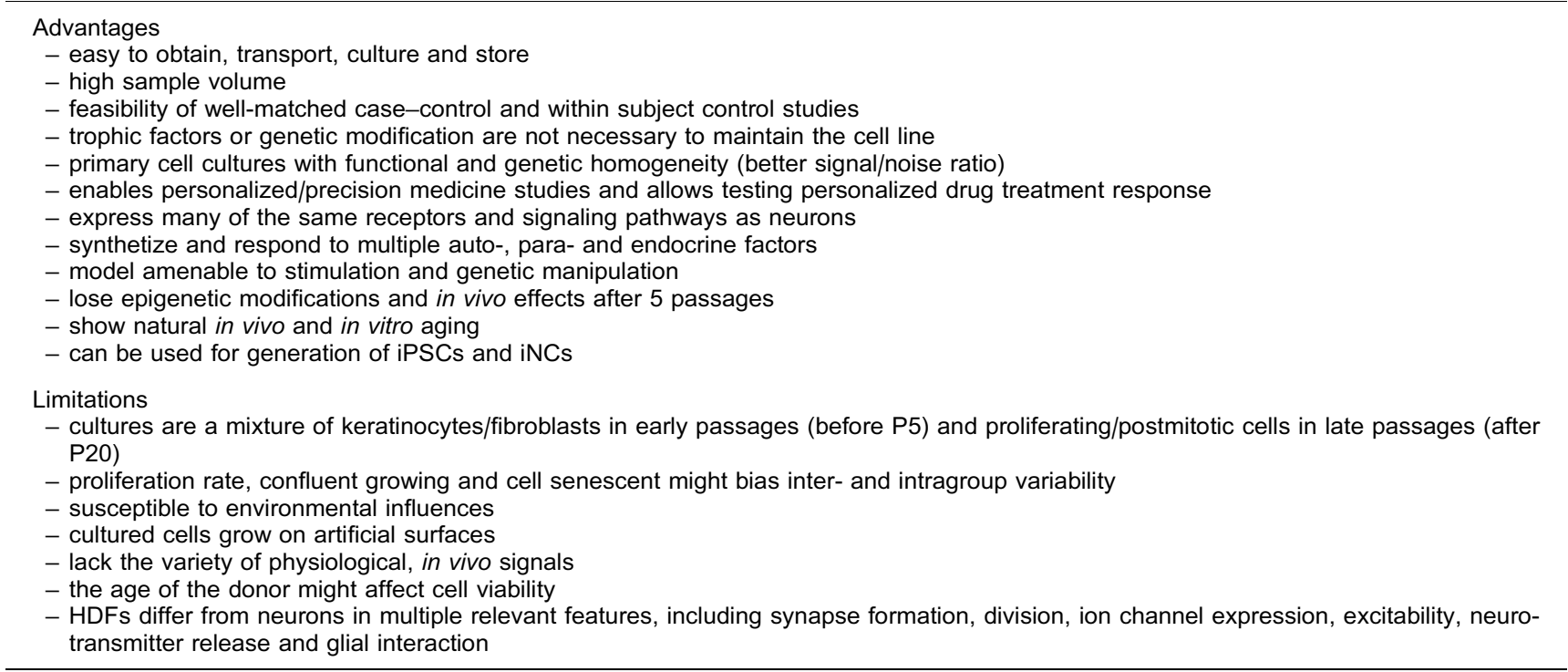

iPSC: induced pluripotent stem cell; iNC: neural cell induced from iPSC or directly from postmitotic somatic cell.

all show individual variability, necessitating careful consideration of experimental and control group size. However, as much as this individual variability represents an experimental challenge in case-control studies, it also allows assessment of individual, patient-specific responses, opening the door to future personalized/precision medicine approaches.

Lastly and obviously, HDF cells are not brain cells, and being aware of the differences between HDFs and neurons is essential when considering the usefulness of the HDF model for a specific experimental purpose: (1) HDFs are much more robust and stress-resilient (Auburger et al., 2012); (2) Unlike post-mitotic neurons, HDFs can proliferate or enter into a reversible quiescent state and then spontaneously regain proliferating capacity (Lemons et al., 2010). (3) HDFs are self-sufficient, while neurons live in a symbiotic relationship with glial cells (Lundgaard et al., 2014); (4) neuronal energy homeostasis relies almost exclusively on lactate oxidation (Lundgaard et al., 2014) while HDFs utilize a much broader range of substrates for metabolic activity (Lemons et al., 2010). (5) There is an undeniable divergence in functional specializations between HDFs and neurons. Neurons are built to be excitable and rapidly convey cell-to-cell signals, while HDFs are primarily stromal cells with a prominent role in immune response and regeneration (Glaros et al., 2009). The advantages and drawbacks of the HDF model are summarized in Table 2.

\section{UTILITY OF FIBROBLASTS IN PSYCHIATRIC RESEARCH}

In summary, the selection of the right in vitro experimental system should always depend on both the characteristic features of the model and the experimental questions asked. In the absence of human neural tissue, HDFs are useful for examining genetically encoded and conserved cellular functions and molecular pathways (e.g. cycle, apoptosis, secretory and intracellular signaling). HDF cultures are easily obtained using minimally invasive skin biopsies; primary cell lines can be established within $24 \mathrm{~h}$ and maintained for more than 20 passages without genetic or chemical manipulation (Akin et al., 2004). Hence, the investigator can perform controlled, reproducible, 'personalized' experiments without a need to alter the individual patient's genetic background. Such a functional model can be used to perform both state and trait experiments, focusing either on the endophenotype at rest, and/or assess resilience or maladaptation to various challenges (and compare the response to that seen in HDF samples obtained from matched controls) (Fig. 1).

Fibroblasts have been traditionally used to study neurodegenerative disorders to investigate mitochondrial dysfunction (Moreira et al., 2007), calcium dynamics (Palotás et al., 2002), membrane composition (Evangelisti et al., 2014), autophagy and lysosomal activation (Coffey et al., 2014), redox homeostasis (Naderi et al., 2006), and amyloid precursor protein or hyperphosphorylated tau- (Shahpasand et al., 2012) related cell damage. In psychiatric research, the late 1980s were the golden age of HDF culture experiments, followed by an extended period of decline in the utilization of this model. This trend is recently changing: in the last 5 years HDFs have been rediscovered as useful patientderived models for studying CNS dysfunction (Fig. 2).

To better understand this trend and to define the areas where HDFs can contribute most to understanding brain disorders, we performed a critical review of the 

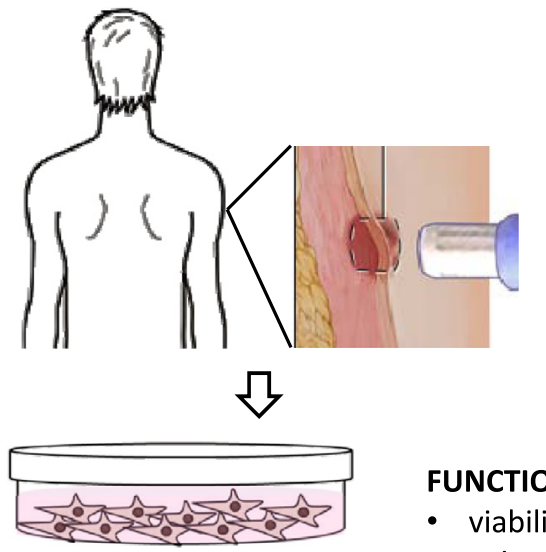

FUNCTIONAL STUDIES

- viability

- aging

"OMICS" STUDIES

- epigenome

- transcriptome

- proteome

- metabolome

\section{DRUG RESPONSE STUDIES}

- case-control studies

- personalized medicine (individual response)

Fig. 1. Utility of human dermal fibroblasts in neuropsychiatric research (iPSC - induced pluripotent stem cell; iNC - induced neural cell; $E C M$ - extracellular matrix; $G^{\star} E$ - gene * environment interaction).

published HDF literature to date. We searched the PubMed database for publications from 1966 until October 2015 using the following keywords: "fibroblast AND SZ" (221 items), "fibroblast AND major depression (MD)" (110 items), "fibroblast AND bipolar disorder (BD)" (81 items), "fibroblast AND autism" (123 items), "fibroblast AND attention deficit" (27 items), "fibroblast AND alcohol dependency" (66 items). Case reports and animal studies were excluded. We also excluded articles when the abstracts did not contain information on study subjects, measured parameters and main findings. It is noteworthy that by design we limited our search to psychiatric disorders and the HDF model - due to the extent of the literature, our review cannot encompass other models and diagnoses (Table 3).

\section{UNDERSTANDING PATHOPHYSIOLOGICAL PROCESSES USING HDFS}

\section{Signaling pathway studies}

Understanding neurotransmitter systems enabled the development of symptom-alleviating drugs in psychiatry, yet it did not elucidate the full pathophysiology of these disorders. This resulted in a shift from studies of neurotransmitter levels to studies of intracellular dysregulations underlying the neurochemical and pathological changes. These studies were quite fruitful and suggest that signal transduction disturbances can lead to altered states of neuronal function and predispose to cognitive and affective disorders (Dwivedi et al., 2002; Akin et al., 2005; Zhan et al., 2011).

The cell-type-specific response to stimuli depends on a cascade of intracellular events, and encompasses receptor and effector expression, the presence of co-regulatory factors, epigenetic modifications and final effectors (most commonly a transcription factor). However, it should be noted that second messenger systems and intracellular signal transduction cascades are highly conserved among many different cell types. Importantly, Rieske et al. (2005) proved that HDF expressed transcription factors, signaling molecules and receptors that are highly similar to cells of neuroectodermal origin (Rieske et al., 2005).

The work of Shelton et al. is a good example of taking advantage of the cellular/ molecular similarity between HDFs and neuronal cells. Studying signal transduction following adrenoreceptor stimulation in HDFs revealed that cAMP-response element-binding protein (CREB) can be activated both through the cAMP/protein kinaseA (PKA) and phospholipase-C/protein kinase C (PLC/ PKC) pathways (Manier et al., 2001). Furthermore, HDFs

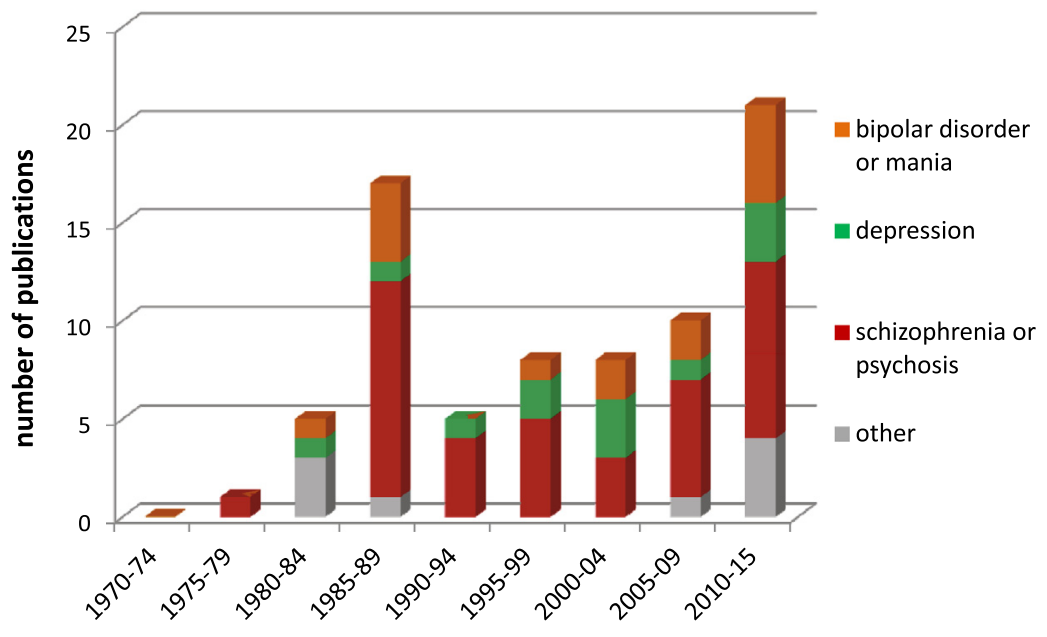

Fig. 2. Number of publications utilizing human dermal fibroblast cultures for studies of psychiatric disorders between 1970 and October 2015 . Note the increased number of publications within the last five years. 
Table 3. Human dermal fibroblast studies in psychiatric disorders

\begin{tabular}{|c|c|c|c|}
\hline Research focus & $\begin{array}{l}\text { Psychiatric } \\
\text { disorder }\end{array}$ & Finding & References \\
\hline \multicolumn{4}{|c|}{ Receptor profile and signaling pathway } \\
\hline \multirow[t]{2}{*}{ Muscarinic cholinergic receptors } & $\begin{array}{l}\text { Affective } \\
\text { disorders }\end{array}$ & $\begin{array}{l}\text { Increased density of binding sites in patients and } \\
\text { their affected or healthy relatives }\end{array}$ & Nadi et al. (1984) \\
\hline & ADHD & Decreased $B_{\max }$ & Johansson et al. $(2013 a, b)$ \\
\hline Serotonin $2 \mathrm{~A}$ receptor & $\mathrm{MD}$ & $\begin{array}{l}\text { Decreased receptor coupled phosphoinositide } \\
\text { response in melancholic MDD }\end{array}$ & Akin et al. (2004) \\
\hline \multirow[t]{2}{*}{$\beta 2$ receptor signaling } & $\mathrm{BD}$ & NSD in receptor coupled cAMP synthesis & Berrettini et al. (1987) \\
\hline & MD & Decreased cAMP induced PKA activity & Shelton et al. (1996) \\
\hline \multirow[t]{4}{*}{ cAMP/PKA signaling } & MD & $\begin{array}{l}\text { Decreased basal and cAMP-stimulated PKA } \\
\text { activity, especially in melancholic subgroup }\end{array}$ & Shelton et al. (1999) \\
\hline & MD & Reduction in the $B_{\max }$ of cAMP binding to PKA & Manier et al. (2000) \\
\hline & $\mathrm{MD}$ & $\begin{array}{l}\text { Reduced PKC dependent CREB phosphorylation; } \\
\text { decreased PKA activity correlated with Hamilton-D } \\
\text { score }\end{array}$ & Akin et al. (2005) \\
\hline & $\mathrm{BD}$ & Increased cAMP induced CREB response & Gaspar et al. (2014) \\
\hline Glucocorticoid receptor & MD & NSD in glucocorticoid binding & Wassef et al. (1992) \\
\hline \multicolumn{4}{|l|}{ Enzyme activity } \\
\hline Transketolase & $\begin{array}{l}\text { AUD and their } \\
\text { offspring }\end{array}$ & Increased $K_{\mathrm{m}}$ & Mukherjee et al. (1987) \\
\hline Kynurenine pathway & $\mathrm{SZ}, \mathrm{BD}$ & $\begin{array}{l}\text { Increased KYNA and 3-HK production; } \\
\text { increased } 3-H K / K Y N A \text { ratio after cytokine; } \\
\text { NSD in enzyme levels }\end{array}$ & Johansson et al. $(2013 a, b)$ \\
\hline \multirow{5}{*}{$\begin{array}{l}\text { Monoamine oxidase-A, -B and } \\
\text { catechol-O-methyl transferase } \\
\text { activity }\end{array}$} & $\mathrm{sZ}$ & NSD & $\begin{array}{l}\text { Groshong et al. (1978), } \\
\text { Ramchand et al. (1995) }\end{array}$ \\
\hline & $\mathrm{BD}$ & NSD & Breakefield et al. (1980) \\
\hline & ASD & NSD & Giller et al. (1980) \\
\hline & $\begin{array}{l}\text { Tourette } \\
\text { syndrome }\end{array}$ & & \\
\hline & AUD & NSD & Giller et al. (1984) \\
\hline \multicolumn{4}{|l|}{ Metabolism } \\
\hline \multirow[t]{3}{*}{ Carbohydrate metabolism } & $\mathrm{SZ}$ & Increased glucose oxidation & Ramchand et al. (1995) \\
\hline & $\begin{array}{l}\text { episodic } \\
\text { schizoaffective } \\
\text { disorder }\end{array}$ & NSD in one-carbon metabolism enzymes & Bruinvels et al. (1988) \\
\hline & $\begin{array}{l}\text { episodic } \\
\text { psychotic } \\
\text { patients }\end{array}$ & NSD in serine-folate metabolism enzyme set & Fekkes and Bruinvels (1986) \\
\hline \multirow[t]{5}{*}{$\begin{array}{l}\text { Cell membrane phospholipid } \\
\text { composition }\end{array}$} & $\mathrm{SZ}$ & $\begin{array}{l}\text { Lower phospholipids, phosphatidylserine, } \\
\text { phosphatidylinositol, phosphatidylethanolamine, } \\
\text { cholesterol fraction }\end{array}$ & Mahadik et al. (1994a) \\
\hline & sz & Increase in polyamines & Ramchand et al. (1994) \\
\hline & $\mathrm{SZ}$ & $\begin{array}{l}\text { Decreased total } n-3 \text { fatty acid; } \\
\text { NSD in arachidonic acid }\end{array}$ & Mahadik et al. (1996a) \\
\hline & $\mathrm{SZ}, \mathrm{BD}$ & $\begin{array}{l}\text { Decreased eicosapentaenoic acid (EPA) and } \\
\text { docosahexaenoic acid (DHA); } \\
\text { NSD in arachidonic acid }\end{array}$ & Ranjekar et al. (2003) \\
\hline & $\mathrm{BD}$ & NSD in total n-3 fatty acids and arachidonic acid & Mahadik et al. (1996a) \\
\hline Utilization of essential fatty acids & $\mathrm{SZ}$ & NSD & Mahadik et al. (1996b) \\
\hline \multicolumn{4}{|l|}{ Mitochondria } \\
\hline $\begin{array}{l}\text { Mitochondrial morphology and } \\
\text { distribution }\end{array}$ & $\mathrm{BD}$ & $\begin{array}{l}\text { Altered mitochondrial morphology and distribution; } \\
\text { abnormal cytochrome C distribution; } \\
\text { NSD in cytochrome C level and ATP production }\end{array}$ & Cataldo et al. (2010) \\
\hline \multicolumn{4}{|l|}{ Membrane transport } \\
\hline \multirow[t]{4}{*}{ Tyrosine } & $\mathrm{SZ}$ & $\begin{array}{l}\text { Decreased } V_{\text {max }} \\
\text { NSD in } K_{m}\end{array}$ & $\begin{array}{l}\text { Hagenfeldt et al. (1987), } \\
\text { Bongiovanni et al. (2013) }\end{array}$ \\
\hline & Sz & Decreased $V_{\max }$ and $K_{\mathrm{m}}$ & $\begin{array}{l}\text { Ramchand et al. (1996), } \\
\text { Flyckt et al. (2001) }\end{array}$ \\
\hline & SZ & Decreased transport due to the L-system & Olsson et al. (2006) \\
\hline & $\mathrm{BD}$ & $\begin{array}{l}\text { Decreased } V_{\max } \\
\text { NSD in } K_{\mathrm{m}}\end{array}$ & Persson et al. (2009) \\
\hline
\end{tabular}


Table 3 (continued)

\begin{tabular}{|c|c|c|c|}
\hline Research focus & $\begin{array}{l}\text { Psychiatric } \\
\text { disorder }\end{array}$ & Finding & References \\
\hline & ADHD & NSD & Johansson et al. $(2011 a, b)$ \\
\hline & ASD & Increased $K_{\mathrm{m}}$ & Fernell et al. (2007) \\
\hline \multirow[t]{2}{*}{ Tryptophan } & ADHD & Decreased $V_{\max }$ and $K_{\mathrm{m}}$ & Johansson et al. $(2011 a, b)$ \\
\hline & $\mathrm{BD}$ & NSC & Persson et al. (2009) \\
\hline Serine, folate & $\begin{array}{l}\text { psychotic } \\
\text { patients }\end{array}$ & NSD in $V_{\max }$ and $K_{\mathrm{m}}$ & Fekkes and Bruinvels (1986) \\
\hline \multirow[t]{2}{*}{ Alanine } & ADHD & Increased $V_{\max }$ & Johansson et al. $(2011 a, b)$ \\
\hline & $\mathrm{AD}$ & Increased $V_{\max }$ & Fernell et al. (2007) \\
\hline Lithium & $\mathrm{BD}, \mathrm{SZ}$ & NSD & $\begin{array}{l}\text { Hitzemann et al. (1988), } \\
\text { Breslow et al. (1985) }\end{array}$ \\
\hline \multicolumn{4}{|l|}{ Cell growth, proliferation and apoptosis } \\
\hline \multirow[t]{4}{*}{ Apoptotic susceptibility } & $\mathrm{SZ}$ & $\begin{array}{l}\text { Increased apoptotic susceptibility; } \\
\text { higher caspase- } 3 \text { activity; } \\
\text { decreased BCL2 }\end{array}$ & Gassó et al. (2014) \\
\hline & $S Z$ & $\begin{array}{l}\text { Increased apoptotic susceptibility; } \\
\text { in vitro apoptotic markers correlate with volumetric } \\
\text { and metabolic brain measures }\end{array}$ & Batalla et al. (2015) \\
\hline & $\mathrm{SZ}$ & $\begin{array}{l}\text { More sub-G0 cells; } \\
\text { attenuated caspase- } 3 \text { response }\end{array}$ & Catts et al. (2006) \\
\hline & $\mathrm{BD}$ & NSD in apoptotic rate & Cataldo et al. (2010) \\
\hline \multirow[t]{4}{*}{ Cell viability, proliferation and growth } & $\mathrm{SZ}$ & NSD & $\begin{array}{l}\text { Cohen et al. (1987), Gassó } \\
\text { et al. (2014) }\end{array}$ \\
\hline & SZ & Reduced growth rate & Mahadik et al. (1991) \\
\hline & SZ & $\begin{array}{l}\text { Cell-cycle associated transcriptome and proteome } \\
\text { changes; } \\
\text { decreased proliferation rate }\end{array}$ & Wang et al. (2010) \\
\hline & $\mathrm{SZ}$ & Decreased adhesiveness and fibronectin synthesis & Mahadik et al. (1994b) \\
\hline Cytoskeleton & $\mathrm{BD}$ & NSD in actin or tubulin cytoskeleton & Cataldo et al. (2010) \\
\hline \multicolumn{4}{|l|}{ Stress resilience and adaptation } \\
\hline \multirow[t]{6}{*}{ Sensitivity to oxidative stress } & $\mathrm{SZ}$ & Impaired capacity to synthesize GSH & $\begin{array}{l}\text { Gysin et al. }(2007,2009) \text {, } \\
\text { Tosic et al. }(2006)\end{array}$ \\
\hline & SZ & $\begin{array}{l}9 \text { protein is affected by the impaired antioxidative } \\
\text { protection in genetically predisposed SCZ patients }\end{array}$ & Gysin et al. (2009) \\
\hline & $\mathrm{SZ}$ & NSD in SOD activity & Cohen et al. (1986) \\
\hline & $\mathrm{MD}$ & $\begin{array}{l}\text { Increased GS reductase; } \\
\text { decreased antioxidant reserve capacity; } \\
\text { NSD in GSH }\end{array}$ & Gibson et al. (2012) \\
\hline & $\mathrm{SZ}$ & $\begin{array}{l}\text { Decreased SOD, CAT and GSH peroxidase; } \\
\text { NSD in membrane lipid peroxidation }\end{array}$ & Ranjekar et al. (2003) \\
\hline & $\mathrm{BD}$ & $\begin{array}{l}\text { Decreased SOD, CAT; } \\
\text { NSD in GP }\end{array}$ & Ranjekar et al. (2003) \\
\hline Metabolic stress response & MD & $\begin{array}{l}26-33 \% \text { of the mRNA and } \\
81-90 \% \text { of the microRNA stress response were } \\
\text { MDD specific }\end{array}$ & Garbett et al., 2015a \\
\hline Metabolome after oxidative stress & Acute psychosis & $\begin{array}{l}\text { Extracellular matrix, arginine metabolism, plasma } \\
\text { membrane alterations; } \\
\text { deficit of lysolipid response }\end{array}$ & Fournier et al. (2014) \\
\hline Sensitivity to DNA-damaging agents & $\mathrm{SZ}, \mathrm{MD}, \mathrm{BD}$ & NSD & Cohen et al. (1989) \\
\hline \multicolumn{4}{|l|}{ Transcriptome } \\
\hline \multirow[t]{4}{*}{ Transcriptome } & $\mathrm{SZ}$ & $\begin{array}{l}434 \text { differentially expressed transcripts, NSD after } \\
\text { multiple comparison test }\end{array}$ & Matigian et al. (2008) \\
\hline & Sz & 6 genes upregulated & Cattane et al. (2015) \\
\hline & MD & $\begin{array}{l}139 \text { genes affected } \\
\text { (114 downregulated); } \\
38 \text { microRNAs changed }\end{array}$ & Garbett et al., $2015 \mathrm{~b}$ \\
\hline & $\begin{array}{l}\text { Fragile } X \\
\text { syndrome }\end{array}$ & $\begin{array}{l}\text { Disease specific protein synthesis and } \\
\text { phosphorylation profile }\end{array}$ & Kumari et al. (2014) \\
\hline
\end{tabular}


Table 3 (continued)

\begin{tabular}{|c|c|c|c|}
\hline Research focus & $\begin{array}{l}\text { Psychiatric } \\
\text { disorder }\end{array}$ & Finding & References \\
\hline \multicolumn{4}{|l|}{ Circadian rhythm } \\
\hline \multirow[t]{4}{*}{ Period length } & $\mathrm{BD}$ & $\begin{array}{l}\text { NSD in period length and clock gene expression } \\
\text { rhythmicity }\end{array}$ & Yang et al. (2009) \\
\hline & $\mathrm{BD}$ & NSD & Bamne et al. (2013) \\
\hline & $\mathrm{BD}$ & $\begin{array}{l}\text { Longer period length; } \\
\text { NSD in amplitude } \\
\text { genotype-determined lithium response }\end{array}$ & McCarthy et al. (2013a) \\
\hline & AUD & $\begin{array}{l}\text { Period correlates inversely with symptom severity; } \\
\text { NSD in contrast with CNT }\end{array}$ & McCarthy et al. (2013b) \\
\hline Lithium-response & BD & $\begin{array}{l}\text { Calcium channel genes modulate the effect of } \\
\text { lithium on the circadian rhythm }\end{array}$ & McCarthy et al. (2015) \\
\hline
\end{tabular}

Abbreviations used: 3-hydroxy-kynurenine (3-HK), attention deficit hyperactivity disorder (ADHD), autism spectrum disorder (ASD), alcohol use disorder (AUD), maximal binding capacity $\left(B_{\max }\right)$, bipolar disorder (BD), cyclic adenosyl monophosphate (cAMP), catalase (CAT), glutathione peroxidase (GP), control, healthy subjects (CNT), glutathione $(\mathrm{GSH})$, substrate affinity $\left(K_{\mathrm{m}}\right)$, kynurenic acid (KYNA), major depressive disorder (MD), no significant difference (NSD), protein kinase A (PKA), schizophrenia $(\mathrm{SZ})$, superoxide dismutase (SOD), maximal transport velocity $\left(V_{\max }\right)$.

from melancholic MD patients had decreased PKA and PKC activity and reduced serotonin $2 A$ receptor-specific PLC response, confirming previous post mortem and platelet findings in MD patients (Shelton et al., 1999; Akin et al., 2004, 2005).

This PKA/PKC-deficit of CREB activation in MD deserves more attention, considering that CREB plays an essential role in activity-driven functional and structural neural changes (Benito and Barco, 2015) and that monoamines, stress hormones and antidepressants affect the CREB/BDNF pro-survival pathway (for a comprehensive review see Dwivedi and Pandey, 2008). Notably, some CREB-mediated cellular functions are neuron-specific, but others - like the circadian rhythm regulation - are also present in peripheral cells (McCarthy et al., 2013a; Druzd et al., 2014; Gaspar et al., 2014).

\section{Circadian rhythm studies}

Disruption of the circadian rhythm is a well-known pathogenic factor for psychiatric disorders such as MD, BD and SZ (Jagannath et al., 2013). During the last decade HDFs have been established as a valid model for studying molecular oscillators, as: (1) cultured HDFs have cell-autonomous circadian rhythm (average length of $24.5 \mathrm{~h}$ ) that depends on the same clock genes found in the pacemaker cells of the suprachiasmatic nuclei (Nagoshi et al., 2004); (2) the circadian rhythm in HDFs is stable, and donor-specific differences are preserved even after multiple cell divisions (Brown et al., 2005; Pagani et al., 2010); and (3) the HDF cellular clock can be re-synchronized with a serum shock $(50 \%$ serum treatment) (Yagita et al., 2001).

In more recent experiments, Gaspar et al. examined the differences in the CAMP/CREB pathway in fibroblast cultures from BD patients and healthy subjects (Gaspar et al., 2014). They found that the CAMP-induced CREB response was inversely correlated with light-stimulated melatonin changes in the donors, and concluded that light-induced melatonin suppression and resetting of the circadian rhythm (Lonze and Ginty, 2002) is present in HDFs. Importantly, BD cells showed approximately 3.3-fold higher CREB induction that control cells, suggesting an increased sensitivity to phase-resetting in BD patients (Gaspar et al., 2014).

Similarly, McCarthy et al. (2013a) found a longer circadian period length in HDF cultures from BD patients. Furthermore, lithium treatment augmented the resynchronization of the circadian clock, with BD responding more prominently to drug-induced resynchronization than the matched control HDFs. Additionally, it was determined that genotype can predict the lithium-induced rhythm changes: increased period length in HDFs was associated with SNPs in the RORA and PER3 genes in a diagnosis-independent fashion. Importantly, SNPs in both of these genes have been previously identified as potential BD, anti-depressant response and post-traumatic stress disorder-associated alleles (Benedetti et al., 2008; Smith et al., 2009; Garriock et al., 2010; Logue et al., 2013).

These experiments suggest that HDFs can be used in studies bridging genetics and disease-associated endophenotypes (i.e. the inherited sensitivity to lightinduced circadian rhythm disruption) (Saini et al., 2015). Furthermore, such assays could sub-stratify affective patients based on circadian rhythm status, and thus open the door for basic and clinical studies that specifically target subgroups of patients.

\section{Studies of stress and adaptation}

Stressful life events contribute to the development of psychiatric disorders (Walker et al., 2008; Musliner et al., 2015). Maladaptive stress response can lead to systemic immune, hormonal and metabolic dysregulation in the body and affective, behavioral and cognitive disturbances in the brain. According to the stress-diathesis or $G^{*} E$ model of $M D$, the hereditary risk factors will manifest in clinical symptoms when the environmental challenges exceed the individual's adaptation potential (Santarelli et al., 2014).

In our recent studies, we used HDF cultures from MD patients and matched controls to determine the genetically encoded stress vulnerability to metabolic 
stimuli (Kálmán et al., 2014; Garbett et al., 2015a,b). mRNA and microRNA profiling analyses after metabolic stress exposure revealed profound transcriptional differences between the MD and control HDFs: the mRNA signature of MD patients consisted of cell cycle, apoptosis and immune function-related gene sets. Furthermore, the metabolic stressors affected MD HDFs differently than controls, as $81-90 \%$ of the microRNA changes were detected only in the MD biomaterial. These findings also suggest that different metabolic stressors lead to different transcriptome changes, thus culturing conditions and stressors for HDFs have to be selected carefully in order to appropriately study the pathophysiology of interest (Kálmán et al., 2014; Garbett et al., 2015a,b).

\section{Redox state studies}

Oxidative stress-related disturbances in SZ, BD and MD are an integral part of these diseases, yet our understanding of the underlying pathophysiology is quite limited (Bajpai et al., 2014; Reyazuddin et al., 2014). As free radicals are found in very low concentrations in human biomaterials and have short half-life (Reyazuddin et al., 2014), the majority of past experiments relied on indirect measures of oxidative stress such as DNA damage, lipid peroxidation, levels of antioxidants and enzyme sets in the blood or the brain tissue (Reyazuddin et al., 2014). Reliable, direct and meaningful measurements of oxidative stress markers are challenging even in freshly prepared plasma due to large differences between intraand extracellular concentrations and rapid molecular transformations (e.g. rapid oxidation, disulfide exchange and redistribution) (Gysin et al., 2011). Thus, functional peripheral assays in HDFs are better suited to assess both the oxidative status and the anti-oxidative reserve capacities of the cells.

A good example of this is the research performed by Do et al. who studied the redox homeostasis in SZ patients by combining neuroimaging, blood and cerebrospinal fluid assessment, genetic testing and HDF functional assays (Do et al., 2000; Tosic et al., 2006; Gysin et al., 2007). Their study, using patient HDFs expanded on previously reported findings that (1) glutamate-cysteine ligase (GCL) is the rate-limiting enzyme of reduced glutathione (GSH) synthesis, which is one of the main antioxidant mechanism across various tissues (Meister, 1995), (2) genetic variants of the GCLMsubunit appear to be associated with greater risk to $S Z$ (Tosic et al., 2006), and (3) in vivo and postmortem studies reported decreased GSH levels in the SZ brain (Do et al., 2000; Yao et al., 2006). The redox homeostasis of SZ HDFs (measured by GSH and GCL levels) was suboptimal even under standard culturing conditions and, when exposed to oxidative stress, HDFs from SZ patients were unable to appropriately adapt and increase GCL activity in response to the environmental challenges (Gysin et al., 2007). Notably, this antioxidant deficit had widespread consequences under stress (Gysin et al., 2009; Fournier et al., 2014) and could also be observed in the blood of patients carrying a high-risk GCL genotype who also had plasma thiol alterations (Gysin et al., 2011).

\section{Age-related studies}

The combined data argue that oxidative stress and inflammation, elevated stress hormones, subsequent insulin resistance, dyslipidemia, premature telomere shortening, mitochondrial dysfunction and multiple other factors contribute to enhanced systemic and brain aging (Wolkowitz et al., 2010; Liu, 2014). At a cellular level, aging is associated with a loss of division potential (replicative senescence) and increased apoptotic rate. Unlike immortal and genetically modified cell line systems the dividing HDFs show natural aging and reflect the biological age of donors, providing a platform to study the effects of genetic and environmental factors on cellular senescence (Tigges et al., 2014).

Although the term 'dementia praecox' is no longer in use (McGlashan, 2011), there are indications that SZ is at least partially a systemic disorder accompanied by accelerated molecular aging (Shivakumar et al., 2014). During the course of the illness, the premature onset of age-related pathophysiological changes mentioned above appear to affect both the brain and the peripheral tissues (reviewed in Kirkpatrick et al., 2008; Shivakumar et al., 2014). Previous results demonstrated accelerated aging-related processes in HDF cultures from SZ patients, which encompassed reduced growth and proliferation rate (Wang et al., 2010), decreased adhesiveness (Mahadik et al., 1994b), relative predominance of sub-G0 cells (Catts et al., 2006), increased apoptotic susceptibility, elevated pro-apoptotic caspase-3 reactivity and lower anti-apoptotic BCl-2 expression (Gassó et al., 2014). These combined findings indicate that the observed molecular similarities between SZ and aging could and should be investigated in HDF cultures, as they may be an important part of the disease pathophysiology (Batalla et al., 2015).

\section{Data-driven studies}

Psychiatric disorders arise as an interplay between the individual's genetic makeup and environmental influences. "Hypothesis-generating" or "data-driven" approaches allow new, unanticipated discoveries, potentially revealing the consequences of known and unknown genetic factors and identifying the most affected pathways and cellular functions.

One example of HDFs used for this purpose is the study by Garbett at al., which provided a detailed description of the transcriptome alterations in HDFs from patients with MD (Garbett et al., 2015a,b). Molecular pathway analysis both on mRNA and microRNA levels revealed that apoptosis, cell-cell communication, adhesion and immune function-related transcripts were significantly altered in the MD samples. These results were consistent with the previous postmortem and in vivo findings (Shelton et al., 2011; Hung et al., 2014), and provided further validation of transcriptome similarities between the brain and peripheral tissue of patients with MD.

The utility of HDFs in data-driven discovery efforts is not solely limited to studies of pathophysiological mechanisms in MD. In a recent analysis, Cattane et al. (2015) performed 
transcriptome profiling of HDFs from SZ patients and controls using DNA microarrays and RT-qPCR. The study identified six differentially expressed genes (Egr1, Fos, Fosb, Hist2h2be, Jun and Tcf4), five of which have been previously implicated in the pathophysiology of SZ in postmortem brain, peripheral blood and genetic studies (Kyosseva, 2004; Kurian et al., 2011; Forrest et al., 2014). It is also notable that this study found that EGR1 levels were correlated in the peripheral blood cells and HDFs from the same donors.

\section{Studies of pharmacological agents}

To date, the majority of studies of psychotropic drugs effects utilized control, immortalized or non-human fibroblasts (e.g. De Filippi et al., 2007; Lauth et al., 2010; Sugden et al., 2010). However, patient HDFs deserve a much greater consideration as models for testing pharmacological agents and responses: (1) In the context of precision/personalized medicine, patientderived HDF lines could be successfully used to predict treatment response to lithium in BP patients (McCarthy et al., 2013a). (2) Self-sustaining HDF cell lines provide a virtually unlimited source of experimental material, enabling high-throughput screening assays for identification of novel therapeutic compounds (Bae et al., 2015; Kubben et al., 2015). (3) This in vitro model system is also useful for identifying unwanted side effects and toxicity of known psychotropic drugs, such as antidepressantcaused cellular damage (Cordero et al., 2009), lithium and valproic acid-caused circadian clock alterations (Johansson et al., 2011a), or antipsychotic agentmodulated aminoacid transport (Bongiovanni et al., 2013).

\section{SIMILARITIES BETWEEN HDF AND CNS CELLS}

Neurons and glia are both affected in psychiatric disorders and both can be considered valid therapeutic targets (Eroglu and Barres, 2010; Sanacora and Banasr, 2013). Interestingly, glia and HDF show striking similarities in several aspects.

(1) Glia generate ECM consisting of hyaluronic acids, glycoproteins and proteoglycans (Faissner et al., 2010). Although ECM is tissue specific, the main components and the cell-matrix dynamics show high similarity throughout various tissues (Naba et al., 2015). The roles of ECM are many: ECM determines structural plasticity and adaptation, modulates inter- and intracellular signaling, ion levels and transmits survival or death/survival signals (Gundelfinger et al., 2010). Postmortem, neuroimaging and genetic studies of SZ, MD and BD all suggest neurodevelopmental disturbances, which might be highly dependent on the extracellular environment (Berretta et al., 2015; Gong and $\mathrm{He}$, 2015; Gurung and Prata, 2015). It has been also suggested that both the composition and the turnover of ECM are likely contributors to the development and progression of psychiatric disorders (Lubbers et al., 2014). For example, the reelin/
Dab1 molecular pathway, which is triggered in the $\mathrm{ECM}$, is implicated in corticogenesis and the neurobiology of SZ and BD (Ovadia and Shifman, 2011; lafrati et al., 2014). Clearly, HDFs can provide valuable information about reelin-induced signal transduction (Leeb et al., 2014), and the similar ECM-generating potential of HDFs and glia might allow mechanistic ECM studies on HDF systems that might provide important disease insights.

(2) Glia provide antioxidant defense in the CNS and closely cooperate with neurons for GSH synthesis (Bélanger et al., 2011). Postmortem and peripheral biomarker studies reveal a diminished ability to maintain redox homeostasis in psychiatric disorders (Bajpai et al., 2014; Reyazuddin et al., 2014). In addition, HDF cultures obtained from SZ and MD patients show impaired GSH regeneration (Cohen et al., 1986; Ranjekar et al., 2003; Tosic et al., 2006; Gysin et al., 2007, 2009; Gibson et al., 2012). Therefore, the decreased oxidative resilience in HDFs is potentially mimicking a dysfunction that occurs in glial cells in the brains of patients with $\mathrm{SZ}$ and $\mathrm{MD}$.

(3) Neurons have a narrow metabolic spectrum and rely on glia in almost every metabolic process (Brown et al., 2014; Lundgaard et al., 2014). Energy storage and supply and lipid homeostasis of the brain (fatty acid oxidation, cholesterol biosynthesis and trafficking) are primarily driven by astrocytes (Dietschy and Turley, 2004; Bélanger et al., 2011). Recent study of HDF from patients with MD revealed a disease-specific response to lipid deprivation (Garbett et al., 2015b), suggesting that this molecular endophenotype might be related to glial cell deficits in subjects with MD.

(4) In addition to microglia, astrocytes also actively participate in the inflammatory and immune response, and they contribute to these processes by secretion of many auto- and paracrine factors (reviewed by Lee and MacLean, 2015). Prolonged neuroinflammation with glial involvement is a key step in the progression of multiple psychiatric diseases (Leonard, 2007; Müller et al., 2012). Consequently, altered expression of immune and cell-cell communication molecular pathways in HDFs from subjects with MD and SZ (Cattane et al., 2015; Garbett et al., $2015 b)$, as well as BD-specific changes in the basal and cytokine-induced tryptophan-kynurenine metabolism (Johansson et al., 2013; Brown et al., 2014) may reflect disrupted glial inflammatory functions in the brains of diseased individuals.

(5) Growth factors coordinate cell proliferation, migration, survival and apoptosis, synapse formation and axonal guidance (Terwisscha et al., 2013). Additionally, two recent meta-analyses showed that serum BDNF is decreased in first episodic psychotic patients (Toll and Mané, 2015) and serum NGF (nerve growth factor) is lowered in MD patients (Chen et al., 2015). Importantly, recent HDF studies by Palazzo et al. (2012) revealed that HDF express BDNF, NGF, neurotrophin-3 (NTF3) and NTF4 and 
their respective receptors. Thus, HDF experiments might allow testing for how genetic variants of growth factors contribute to acquired vulnerability to psychiatric disorders.

\section{CONCLUSIONS}

The expanding literature during the last several decades suggests that the patient-derived HDF model is a valuable platform for developing novel molecular biology methods and represents a useful cellular system for studying the molecular basis of psychiatric disorders. The value of HDFs is based on ease of acquiring/ establishing of the cell lines from patients, sharing molecular similarities with cells from the CNS, and the ability to be propagated without additional genetic manipulations. HDFs also facilitate our understanding of brain disorders by allowing us to examine similarities between the in vitro observations and pathophysiology of the CNS, and by providing the arena for investigating $\mathrm{G}^{*} \mathrm{E}$ interactions, signal transduction and metabolic dysfunctions. Additionally, the HDF model can be instrumental in identifying brain disease biomarkers and thus emphasize the organic aspects of neuropsychiatric disorders. Due to these characteristics, in the near future HDFs might provide seminal contribution to pharmacogenetics and individual therapeutic strategies used in precision/personalized medicine.

Acknowledgements_K.M.'s work was partially supported by R01 MH079299 (NIMH). S.K.'s work was partially supported by the Vanderbilt International Summer Research Academy (VISRA) scholarship and the Vanderbilt International Scholar Short-Term Visit Fellowship. We are grateful to Ms. Kelli Money for editing and providing insightful comments on the manuscript.

\section{REFERENCES}

Akin D, Manier DH, Sanders-Bush E, Shelton RC (2004) Decreased serotonin 5-HT2A receptor-stimulated phosphoinositide signaling in fibroblasts from melancholic depressed patients. Neuropsychopharmacology 29:2081-2087.

Akin D, Manier DH, Sanders-Bush E, Shelton RC (2005) Signal transduction abnormalities in melancholic depression. Int $J$ Neuropsychopharmacol 8(1):5-16.

Antequera F, Boyes J, Bird A (1990) High levels of de novo methylation and altered chromatin structure at $\mathrm{CpG}$ islands in cell lines. Cell 62(3):503-514.

Auburger G, Klinkenberg M, Drost J, Marcus K, Morales-Gordo B, Kunz WS, Brandt U, Broccoli V, Reichmann H, Gispert S, Jendrach M (2012) Primary skin fibroblasts as a model of Parkinson's disease. Mol Neurobiol 46(1):20-27.

Bae S, An IS, An S (2015) Development of a high-throughput screening system for identification of novel reagents regulating DNA damage in human dermal fibroblasts. Acta Pharm 65 (3):331-341.

Bajpai A, Verma AK, Srivastava M, Srivastava R (2014) Oxidative stress and major depression. J Clin Diagn Res 8(12): CC04-CC07.

Bamne MN, Ponder CA, Wood JA, Mansour H, Frank E, Kupfer DJ, Young MW, Nimgaonkar VL (2013) Application of an ex vivo cellular model of circadian variation for bipolar disorder research: a proof of concept study. Bipolar Disord 15(6):694-700.

Batalla A, Bargalló N, Gassó P, Molina O, Pareto D, Mas S, Roca JM, Bernardo M, Lafuente A, Parellada E (2015) Apoptotic markers in cultured fibroblasts correlate with brain metabolites and regional brain volume in antipsychotic-naïve first-episode schizophrenia and healthy controls. Transl Psychiatry 5:e626.

Bayreuther K, Francz PI, Gogol J, Hapke C, Maier M, Meinrath HG (1991) Differentiation of primary and secondary fibroblasts in cell culture systems. Mutat Res 256(2-6):233-242.

Bélanger M, Allaman I, Magistretti PJ (2011) Brain energy metabolism: focus on astrocyte-neuron metabolic cooperation. Cell Metab 14(6):724-738.

Belinsky GS, Rich MT, Sirois CL, Short SM, Pedrosa E, Lachman HM, Antic SD (2014) Patch-clamp recordings and calcium imaging followed by single-cell PCR reveal the developmental profile of 13 genes in iPSC-derived human neurons. Stem Cell Res 12(1):101-118.

Benedetti F, Dallaspezia S, Colombo C, Pirovano A, Marino E, Smeraldi E (2008) A length polymorphism in the circadian clock gene Per3 influences age at onset of bipolar disorder. Neurosci Lett 445(2):184-187.

Benito E, Barco A (2015) The neuronal activity-driven transcriptome. Mol Neurobiol 51(3):1071-1088.

Berretta S, Pantazopoulos H, Markota M, Brown C, Batzianouli ET (2015) Losing the sugar coating: Potential impact of perineuronal net abnormalities on interneurons in schizophrenia. Schizophr Res 167(1-3):18-27.

Berrettini WH, Bardakjian J, Cappellari CB, Barnett Jr AL, Albright A, Nurnberger Jr JI, Gershon ES (1987) Skin fibroblast betaadrenergic receptor function in manic-depressive illness. Biol Psychiatry 22(12):1439-1443.

Biran R, Noble MD, Tresco PA (1999) Characterization of cortical astrocytes on materials of differing surface chemistry. J Biomed Mater Res 46(2):150-159.

Bongiovanni R, Leonard S, Jaskiw GE (2013) A simplified method to quantify dysregulated tyrosine transport in schizophrenia. Schizophr Res 150(2-3):386-391.

Borgmann-Winter K, Willard SL, Sinclair D, Mirza N, Turetsky B, Berretta S, Hahn CG (2015) Translational potential of olfactory mucosa for the study of neuropsychiatric illness. Transl Psychiatry 5:e527.

Breakefield XO, Giller Jr EL, Nurnberger Jr JI, Castiglione CM, Buchsbaum MS, Gershon ES (1980) Monoamine oxidase type A in fibroblasts from patients with bipolar depressive illness. Psychiatry Res 2(3):307-314.

Brennand KJ, Simone A, Tran N, Gage FH (2012) Modeling psychiatric disorders at the cellular and network levels. Mol Psychiatry 17(12):1239-1253.

Brennand KJ, Landek-Salgado MA, Sawa A (2014) Modeling heterogeneous patients with a clinical diagnosis of schizophrenia with induced pluripotent stem cells. Biol Psychiatry 75(12):936-944.

Breslow RE, DeMuth GW, Weiss C (1985) Lithium incorporation in the fibroblasts of manic-depressives. Biol Psychiatry 20(1):58-65.

Brown SA, Fleury-Olela F, Nagoshi E, Hauser C, Juge C, Meier CA, Chicheportiche R, Dayer JM, Albrecht U, Schibler U (2005) The period length of fibroblast circadian gene expression varies widely among human individuals. PLoS Biol 3(10):e338.

Brown JA, Sherrod SD, Goodwin CR, Brewer B, Yang L, Garbett KA, Li D, McLean JA, Wikswo JP, Mirnics K (2014) Metabolic consequences of interleukin-6 challenge in developing neurons and astroglia. J Neuroinflamm 11:183.

Brown JA, Ramikie TS, Schmidt MJ, Báldi R, Garbett K, Everheart MG, Warren LE, Gellért L, Horváth S, Patel S, Mirnics K (2015) Inhibition of parvalbumin-expressing interneurons results in complex behavioral changes. Mol Psychiatry.

Bruinvels J, Pepplinkhuizen L, Fekkes D (1988) Derangement of onecarbon metabolism in episodic schizoaffective psychoses. Pharm Psychiatry 21(1):28-32.

Cataldo AM, McPhie DL, Lange NT, Punzell S, Elmiligy S, Ye NZ, Froimowitz MP, Hassinger LC, Menesale EB, Sargent LW, Logan DJ, Carpenter AE, Cohen BM (2010) Abnormalities in mitochondrial structure in cells from patients with bipolar disorder. Am J Pathol 177(2):575-585. 
Cattane N, Minelli A, Milanesi E, Maj C, Bignotti S, Bortolomasi M, Bocchio Chiavetto L, Gennarelli M (2015) Altered gene expression in schizophrenia: findings from transcriptional signatures in fibroblasts and blood. PLoS One 10(2):e0116686.

Catts VS, Catts SV, McGrath JJ, Féron F, McLean D, Coulson EJ, Lutze-Mann LH (2006) Apoptosis and schizophrenia: a pilot study based on dermal fibroblast cell lines. Schizophr Res 84 (1):20-28

Chanda S, Ang CE, Davila J, Pak C, Mall M, Lee QY, Ahlenius H, Jung SW, Südhof TC, Wernig M (2014) Generation of induced neuronal cells by the single reprogramming factor ASCL1. Stem Cell Rep 3(2):282-296.

Chen YW, Lin PY, Tu KY, Cheng YS, Wu CK, Tseng PT (2015) Significantly lower nerve growth factor levels in patients with major depressive disorder than in healthy subjects: a metaanalysis and systematic review. Neuropsychiatry Dis Treat 11:925-933.

Coffey EE, Beckel JM, Laties AM, Mitchell CH (2014) Lysosomal alkalization and dysfunction in human fibroblasts with the Alzheimer's disease-linked presenilin 1 A246E mutation can be reversed with cAMP. Neuroscience 263:111-124.

Cohen MR, Sailer V, McAmis B, Jenkins P (1986) Superoxide dismutase activity in fibroblasts from patients with schizophrenia. Biol Psychiatry 21(3):322-324.

Cohen MR, Gutman R, McAmis W (1987) Cultured skin fibroblasts in schizophrenia: acute growth and susceptibility to damage. Psychiatry Res 21(1):43-47.

Cohen MR, McAmis W, Gutman R (1989) Sensitivity to N-methyl-N'nitro-N-nitrosoguanidine in fibroblasts from patients with schizophrenia. Biol Psychiatry 25(2):239-242.

Connolly GP (1998) Fibroblast models of neurological disorders: fluorescence measurement studies. Trends Pharmacol Sci 19(5): $171-177$.

Cordero MD, Moreno-Fernández AM, Gomez-Skarmeta JL, de Miguel M, Garrido-Maraver J, Oropesa-Avila M, RodríguezHernández A, Navas P, Sánchez-Alcázar JA (2009) Coenzyme Q10 and alpha-tocopherol protect against amitriptyline toxicity. Toxicol Appl Pharmacol 235(3):329-337.

Corrales NL, Mrasek K, Voigt M, Liehr T, Kosyakova N (2012) Copy number variations (CNVs) in human pluripotent cell-derived neuroprogenitors. Gene 506(2):377-379.

Dage JL, Colvin EM, Fouillet A, Langron E, Roell WC, Li J, Mathur SX, Mogg AJ, Schmitt MG, Felder CC, Merchant KM, Isaac J, Broad LM, Sher E, Ursu D (2014) Pharmacological characterisation of ligand- and voltage-gated ion channels expressed in human iPSC-derived forebrain neurons. Psychopharmacology 231(6):1105-1124.

De Filippi L, Fournier M, Cameroni E, Linder P, De Virgilio C, Foti M, Deloche O (2007) Membrane stress is coupled to a rapid translational control of gene expression in chlorpromazinetreated cells. Curr Genet 52(3-4):171-185.

Dietschy JM, Turley SD (2004) Thematic review series: brain Lipids. Cholesterol metabolism in the central nervous system during early development and in the mature animal. J Lipid Res 45(8): 1375-1397.

Dimos JT, Rodolfa KT, Niakan KK, Weisenthal LM, Mitsumoto H, Chung W, Croft GF, Saphier G, Leibel R, Goland R, Wichterle H, Henderson CE, Eggan K (2008) Induced pluripotent stem cells generated from patients with ALS can be differentiated into motor neurons. Science 321(5893):1218-1221.

Do KQ, Trabesinger AH, Kirsten-Krüger M, Lauer CJ, Dydak U, Hell D, Holsboer F, Boesiger P, Cuénod M (2000) Schizophrenia: glutathione deficit in cerebrospinal fluid and prefrontal cortex in vivo. Eur J Neurosci 12(10):3721-3728.

Druzd D, de Juan A, Scheiermann C (2014) Circadian rhythms in leukocyte trafficking. Semin Immunopathol. 36(2):149-162.

Dwivedi Y, Pandey GN (2008) Adenylyl cyclase-cyclicAMP signaling in mood disorders: role of the crucial phosphorylating enzyme protein kinase A. Neuropsychiatry Dis Treat 4(1):161-176.

Dwivedi Y, Conley RR, Roberts RC, Tamminga CA, Pandey GN (2002) [(3)H]cAMP binding sites and protein kinase a activity in the prefrontal cortex of suicide victims. Am J Psychiatry 159(1): 66-73.

Ebert AD, Yu J, Rose Jr FF, Mattis VB, Lorson CL, Thomson JA, Svendsen CN (2009) Induced pluripotent stem cells from a spinal muscular atrophy patient. Nature 457(7227):277-280.

Eroglu C, Barres BA (2010) Regulation of synaptic connectivity by glia. Nature 468(7321):223-231.

Evangelisti E, Zampagni M, Cascella R, Becatti M, Fiorillo C, Caselli A, Bagnoli S, Nacmias B, Cecchi C (2014) Plasma membrane injury depends on bilayer lipid composition in Alzheimer's disease. J Alzheimers Dis 41(1):289-300.

Faissner A, Pyka M, Geissler M, Sobik T, Frischknecht R, Gundelfinger ED, Seidenbecher C (2010) Contributions of astrocytes to synapse formation and maturation - Potential functions of the perisynaptic extracellular matrix. Brain Res Rev $63(1-2): 26-38$.

Fathi A, Hatami M, Hajihosseini V, Fattahi F, Kiani S, Baharvand H, Salekdeh GH (2011) Comprehensive gene expression analysis of human embryonic stem cells during differentiation into neural cells. PLoS One 6(7):e22856.

Fekkes D, Bruinvels J (1986) Serine and folate metabolism in fibroblasts from episodic psychotic patients with psychedelic symptoms. Biol Psychiatry 21(10):951-959.

Fernell E, Karagiannakis A, Edman G, Bjerkenstedt L, Wiesel FA, Venizelos N (2007) Aberrant amino acid transport in fibroblasts from children with autism. Neurosci Lett 418(1):82-86.

Féron F, Perry C, Girard SD, Mackay-Sim A (2013) Isolation of adult stem cells from the human olfactory mucosa. Methods Mol Biol 1059:107-114.

Flyckt L, Venizelos N, Edman G, Bjerkenstedt L, Hagenfeldt L, Wiesel FA (2001) Aberrant tyrosine transport across the cell membrane in patients with schizophrenia. Arch Gen Psychiatry 58(10): 953-958.

Forrest MP, Hill MJ, Quantock AJ, Martin-Rendon E, Blake DJ (2014) The emerging roles of TCF4 in disease and development. Trends Mol Med 20(6):322-331.

Fournier M, Ferrari C, Baumann PS, Polari A, Monin A, BellierTeichmann T, Wulff J, Pappan KL, Cuenod M, Conus P, Do KQ (2014) Impaired metabolic reactivity to oxidative stress in early psychosis patients. Schizophr Bull 40(5):973-983.

Fridman AL, Tainsky MA (2008) Critical pathways in cellular senescence and immortalization revealed by gene expression profiling. Oncogene 27(46):5975-5987.

Fullár A, Dudás J, Oláh L, Hollósi P, Papp Z, Sobel G, Karászi K, Paku S, Baghy K, Kovalszky I (2015) Remodeling of extracellular matrix by normal and tumor-associated fibroblasts promotes cervical cancer progression. BMC Cancer 15:256.

Garbett KA, Vereczkei A, Kálmán S, Wang L, Korade Ž, Shelton RC, Mirnics K (2015a) Fibroblasts from patients with major depressive disorder show distinct transcriptional response to metabolic stressors. Transl Psychiatry 5:e523.

Garbett KA, Vereczkei A, Kálmán S, Brown JA, Taylor WD, Faludi G, Korade Ž, Shelton RC, Mirnics K (2015b) Coordinated messenger RNA/microRNA changes in fibroblasts of patients with major depression. Biol Psychiatry 77(3):256-265.

Garriock HA, Kraft JB, Shyn SI, Peters EJ, Yokoyama JS, Jenkins GD, Reinalda MS, Slager SL, McGrath PJ, Hamilton SP (2010) A genomewide association study of citalopram response in major depressive disorder. Biol Psychiatry 67(2):133-138.

Gaspar L, van de Werken M, Johansson AS, Moriggi E, Owe-Larsson B, Kocks JW, Lundkvist GB, Gordijn MC, Brown SA (2014) Human cellular differences in CAMP-CREB signaling correlate with light-dependent melatonin suppression and bipolar disorder. Eur J Neurosci 40(1):2206-2215.

Gassó P, Mas S, Molina O, Lafuente A, Bernardo M, Parellada E (2014) Increased susceptibility to apoptosis in cultured fibroblasts from antipsychotic-naïve first-episode schizophrenia patients. J Psychiatry Res 48(1):94-101.

Gibson SA, Korade Z, Shelton RC (2012) Oxidative stress and glutathione response in tissue cultures from persons with major depression. J Psychiatry Res 46(10):1326-1332. 
Giller Jr EL, Young JG, Breakefield XO, Carbonari C, Braverman M, Cohen DJ (1980) Monoamine oxidase and catechol-Omethyltransferase activities in cultured fibroblasts and blood cells from children with autism and the Gilles de la Tourette syndrome. Psychiatry Res 2(2):187-197.

Giller Jr E, Nocks J, Hall H, Stewart C, Schnitt J, Sherman B (1984) Platelet and fibroblast monoamine oxidase in alcoholism. Psychiatry Res 12(4):339-347.

Gladkevich A, Kauffman HF, Korf J (2004) Lymphocytes as a neural probe: potential for studying psychiatric disorders. Prog Neuropsychopharmacol Biol Psychiatry 28(3):559-576.

Glaros T, Larsen M, Li L (2009) Macrophages and fibroblasts during inflammation, tissue damage and organ injury. Front Biosci (Landmark Ed) 14:3988-3993.

Gomez G, Rawson NE, Hahn CG, Michaels R, Restrepo D (2008) Characteristics of odorant elicited calcium changes in cultured human olfactory neurons. J Neurosci Res 62(5):737-749.

Gong Q, He Y (2015) Depression, neuroimaging and connectomics: a selective overview. Biol Psychiatry 77(3):223-235.

Greiner JF, Hauser S, Widera D, Müller J, Qunneis F, Zander C, Martin I, Mallah J, Schuetzmann D, Prante C, Schwarze H, Prohaska W, Beyer A, Rott K, Hütten A, Gölzhäuser A, Sudhoff H, Kaltschmidt C, Kaltschmidt B (2011) Efficient animal-serum free 3D cultivation method for adult human neural crest-derived stem cell therapeutics. Eur Cell Mater 22:403-419.

Groshong R, Baldessarini RJ, Gibson DA, Lipinski JF, Axelrod D, Pope A (1978) Activities of types A and B MAO and catechol-omethyltransferase in blood cells and skin fibroblasts of normal and chronic schizophrenic subjects. Arch Gen Psychiatry 35 (10):1198-1205.

Gundelfinger ED, Frischknecht R, Choquet D, Heine M (2010) Converting juvenile into adult plasticity: a role for the brain's extracellular matrix. Eur J Neurosci 31(12):2156-2165.

Gurung R, Prata DP (2015) What is the impact of genome-wide supported risk variants for schizophrenia and bipolar disorder on brain structure and function? A systematic review. Psychol Med 10:1-20.

Gysin R, Kraftsik R, Sandell J, Bovet P, Chappuis C, Conus P, Deppen P, Preisig M, Ruiz V, Steullet P, Tosic M, Werge T, Cuénod M, Do KQ (2007) Impaired glutathione synthesis in schizophrenia: convergent genetic and functional evidence. Proc Natl Acad Sci U S A 104(42):16621-16626.

Gysin R, Riederer IM, Cuénod M, Do KQ, Riederer BM (2009) Skin fibroblast model to study an impaired glutathione synthesis: consequences of a genetic polymorphism on the proteome. Brain Res Bull 79(1):46-52.

Gysin R, Kraftsik R, Boulat O, Bovet P, Conus P, Comte-Krieger E, Polari A, Steullet P, Preisig M, Teichmann T, Cuénod M, Do KQ (2011) Genetic dysregulation of glutathione synthesis predicts alteration of plasma thiol redox status in schizophrenia. Antioxid Redox Signal 15(7):2003-2010.

Hagenfeldt L, Venizelos N, Bjerkenstedt L, Wiesel FA (1987) Decreased tyrosine transport in fibroblasts from schizophrenic patients. Life Sci 41(25):2749-2757.

Hänzelmann S, Beier F, Gusmao EG, Koch CM, Hummel S, Charapitsa I, Joussen S, Benes V, Brümmendorf TH, Reid G, Costa IG, Wagner W (2015) Replicative senescence is associated with nuclear reorganization and with DNA methylation at specific transcription factor binding sites. Clin Epigenet 7(1):19.

Hayashi-Takagi A, Vawter MP, Iwamoto K (2014) Peripheral biomarkers revisited: integrative profiling of peripheral samples for psychiatric research. Biol Psychiatry 75(12):920-928.

Hitzemann R, Kao L, Hirschowitz J, Garver D, Gruenstein E (1988) Lithium transport in human fibroblasts: relationship to RBC lithium transport and psychiatric diagnoses. Psychiatry Res 24(3): 337-344.

Horváth S, Mirnics K (2015) Schizophrenia as a disorder of molecular pathways. Biol Psychiatry 77(1):22-28.

Horváth S, Janka Z, Mirnics K (2011) Analyzing schizophrenia by DNA microarrays. Biol Psychiatry 69(2):157-162.
Hu BY, Weick JP, Yu J, Ma LX, Zhang XQ, Thomson JA, Zhang SC (2010) Neural differentiation of human induced pluripotent stem cells follows developmental principles but with variable potency. Proc Natl Acad Sci U S A 107(9):4335-4340.

Huang HM, Fowler C, Xu H, Zhang H, Gibson GE (2005) Mitochondrial function in fibroblasts with aging in culture and/or Alzheimer's disease. Neurobiol Aging 26(6):839-848.

Huard JM, Youngentob SL, Goldstein BJ, Luskin MB, Schwob JE (1998) Adult olfactory epithelium contains multipotent progenitors that give rise to neurons and non-neural cells. J Comp Neurol 400 (4):469-486.

Hung YY, Kang HY, Huang KW, Huang TL (2014) Association between toll-like receptors expression and major depressive disorder. Psychiatry Res 220(1-2):283-286.

lafrati J, Orejarena MJ, Lassalle O, Bouamrane L, Gonzalez-Campo C, Chavis P (2014) Reelin, an extracellular matrix protein linked to early onset psychiatric diseases, drives postnatal development of the prefrontal cortex via GluN2B-NMDARsand the mTOR pathway. Mol Psychiatry 19(4):417-426.

Iga J, Ueno S, Ohmori T (2008) Molecular assessment of depression from mRNAs in the peripheral leukocytes. Ann Med 40(5): 336-342.

Israel MA, Yuan SH, Bardy C, Reyna SM, Mu Y, Herrera C, Hefferan MP, Van Gorp S, Nazor KL, Boscolo FS, Carson CT, Laurent LC, Marsala M, Gage FH, Remes AM, Koo EH, Goldstein LS (2012) Probing sporadic and familial Alzheimer's disease using induced pluripotent stem cells. Nature 482(7384):216-220.

Jagannath A, Peirson SN, Foster RG (2013) Sleep and circadian rhythm disruption in neuropsychiatric illness. Curr Opin Neurobiol 23(5):888-894.

Johansson AS, Brask J, Owe-Larsson B, Hetta J, Lundkvist GB (2011a) Valproic acid phase shifts the rhythmic expression of Period2::Luciferase. J Biol Rhythms 26(6):541-551.

Johansson J, Landgren M, Fernell E, Vumma R, Åhlin A, Bjerkenstedt L, Venizelos N (2011b) Altered tryptophan and alanine transport in fibroblasts from boys with attention-deficit/ hyperactivity disorder (ADHD): an in vitro study. Behav Brain Funct 7:40.

Johansson AS, Owe-Larsson B, Asp L, Kocki T, Adler M, Hetta J, Gardner R, Lundkvist GB, Urbanska EM, Karlsson H (2013a) Activation of kynurenine pathway in ex vivo fibroblasts from patients with bipolar disorder or schizophrenia: cytokine challenge increases production of 3-hydroxykynurenine. J Psychiatry Res 47(11):1815-1823.

Johansson J, Landgren M, Fernell E, Lewander T, Venizelos N (2013b) Decreased binding capacity (Bmax) of muscarinic acetylcholine receptors in fibroblasts from boys with attentiondeficit/hyperactivity disorder (ADHD). Atten Def Hyperact Disord 5 (3):267-271.

Kaiser T, Feng G (2015) Modeling psychiatric disorders for developing effective treatments. Nat Med 21(9):979-988.

Kálmán S, Garbett KA, Vereczkei A, Shelton RC, Korade Z, Mirnics K (2014) Metabolic stress-induced microRNA and mRNA expression profiles of human fibroblasts. Exp Cell Res 320(2): 343-353.

Khandaker GM, Dantzer R (2015) Is there a role for immune-to-brain communication in schizophrenia? Psychopharmacology.

Kim DS, Ross PJ, Zaslavsky K, Ellis J (2014) Optimizing neuronal differentiation from induced pluripotent stem cells to model ASD. Front Cell Neurosci 8:109.

Kirkpatrick B, Messias E, Harvey PD, Fernandez-Egea E, Bowie CR (2008) Is schizophrenia a syndrome of accelerated aging? Schizophr Bull 34(6):1024-1032.

Koch CM, Suschek CV, Lin Q, Bork S, Goergens M, Joussen S, Pallua N, Ho AD, Zenke M, Wagner W (2011) Specific ageassociated DNA methylation changes in human dermal fibroblasts. PLoS One 6(2):e16679.

Krieger K, Klimke A, Henning U (1998) Active [3H]-dopamine uptake displayed by native lymphocyte suspensions is mainly due to contaminating platelets. Pharmacopsychiatry 31(5):193-198. 
Kubben N, Brimacombe KR, Donegan M, Li Z, Misteli T (2015) A high-content imaging-based screening pipeline for the systematic identification of anti-progeroid compounds. Methods. pii: S10462023(15)30070-0.

Kumari D, Bhattacharya A, Nadel J, Moulton K, Zeak NM, Glicksman A, Dobkin C, Brick DJ, Schwartz PH, Smith CB, Klann E, Usdin K (2014) Identification of fragile $X$ syndrome specific molecular markers in human fibroblasts: a useful model to test the efficacy of therapeutic drugs. Hum Mutat 35(12):1485-1494.

Kurian SM, Le-Niculescu H, Patel SD, Bertram D, Davis J, Dike C, Yehyawi N, Lysaker P, Dustin J, Caligiuri M, Lohr J, Lahiri DK, Nurnberger Jr JI, Faraone SV, Geyer MA, Tsuang MT, Schork NJ, Salomon DR, Niculescu AB (2011) Identification of blood biomarkers for psychosis using convergent functional genomics. Mol Psychiatry 16(1):37-58.

Kyosseva SV (2004) Differential expression of mitogen-activated protein kinases and immediate early genes fos and jun in thalamus in schizophrenia. Prog Neuropsychopharmacol Biol Psychiatry 28(6):997-1006.

Lauth M, Rohnalter V, Bergström A, Kooshesh M, Svenningsson P, Toftgård R (2010) Antipsychotic drugs regulate hedgehog signaling by modulation of 7-dehydrocholesterol reductase levels. Mol Pharmacol 78(3):486-496.

Lee KM, MacLean AG (2015) New advances on glial activation in health and disease. World J Virol 4(2):42-55.

Leeb C, Eresheim C, Nimpf J (2014) Clusterin is a ligand for apolipoprotein E receptor 2 (ApoER2) and very low density lipoprotein receptor (VLDLR) and signals via the Reelin-signaling pathway. J Biol Chem 289(7):4161-4172.

Lemons JM, Feng XJ, Bennett BD, Legesse-Miller A, Johnson EL, Raitman I, Pollina EA, Rabitz HA, Rabinowitz JD, Coller HA (2010) Quiescent fibroblasts exhibit high metabolic activity. PLoS Biol 8(10):e1000514.

Leonard BE (2007) Inflammation, depression and dementia: are they connected? Neurochem Res 32(10):1749-1756.

Liu JP (2014) Molecular mechanisms of ageing and related diseases. Clin Exp Pharmacol Physiol 41(7):445-458.

Liu Y, Liu H, Sauvey C, Yao L, Zarnowska ED, Zhang SC (2013) Directed differentiation of forebrain GABA interneurons from human pluripotent stem cells. Nat Protoc 8(9):1670-1679.

Logue MW, Baldwin C, Guffanti G, Melista E, Wolf EJ, Reardon AF, Uddin M, Wildman D, Galea S, Koenen KC, Miller MW (2013) A genome-wide association study of post-traumatic stress disorder identifies the retinoid-related orphan receptor alpha (RORA) gene as a significant risk locus. Mol Psychiatry 18(8):937-942.

Lonze BE, Ginty DD (2002) Function and regulation of CREB family transcription factors in the nervous system. Neuron 35(4): 605-623.

Lu P, Takai K, Weaver VM, Werb Z (2011) Extracellular matrix degradation and remodeling in development and disease. Cold Spring Harb Perspect Biol 3(12). pii: a005058.

Lubbers BR, Smit AB, Spijker S, van den Oever MC (2014) Neural ECM in addiction, schizophrenia, and mood disorder. Prog Brain Res 214:263-284.

Lundgaard I, Osório MJ, Kress BT, Sanggaard S, Nedergaard M (2014) White matter astrocytes in health and disease. Neuroscience 276:161-173.

Mahadik SP, Mukherjee S, Laev H, Reddy R, Schnur DB (1991) Abnormal growth of skin fibroblasts from schizophrenic patients. Psychiatry Res 37(3):309-320.

Mahadik SP, Mukherjee S, Correnti EE, Kelkar HS, Wakade CG, Costa RM, Scheffer R (1994a) Plasma membrane phospholipid and cholesterol distribution of skin fibroblasts from drug-naive patients at the onset of psychosis. Schizophr Res 13(3):239-247.

Mahadik SP, Mukherjee S, Wakade CG, Laev H, Reddy RR, Schnur DB (1994b) Decreased adhesiveness and altered cellular distribution of fibronectin in fibroblasts from schizophrenic patients. Psychiatry Res 53(1):87-97.

Mahadik SP, Mukherjee S, Horrobin DF, Jenkins K, Correnti EE, Scheffer RE (1996a) Plasma membrane phospholipid fatty acid composition of cultured skin fibroblasts from schizophrenic patients: comparison with bipolar patients and normal subjects. Psychiatry Res 63(2-3):133-142.

Mahadik SP, Shendarkar NS, Scheffer RE, Mukherjee S, Correnti EE (1996b) Utilization of precursor essential fatty acids in culture by skin fibroblasts from schizophrenic patients and normal controls. Prostaglandins Leukot Essent Fatty Acids 55(1-2):65-70.

Maier AB, le Cessie S, de Koning-Treurniet C, Blom J, Westendorp RG, van Heemst D (2007) Persistence of high-replicative capacity in cultured fibroblasts from nonagenarians. Aging Cell 6(1):27-33.

Manier DH, Shelton RC, Ellis TC, Peterson CS, Eiring A, Sulser F (2000) Human fibroblasts as a relevant model to study signal transduction in affective disorders. J Affect Disord 61(1-2):51-58.

Manier DH, Shelton RC, Sulser F (2001) Cross-talk between PKA and PKC in human fibroblasts: what are the pharmacotherapeutic implications? J Affect Disord 65(3):275-279.

Martins-Taylor K, Xu RH (2012) Concise review: Genomic stability of human induced pluripotent stem cells. Stem Cells 30(1):22-27.

Matigian NA, McCurdy RD, Féron F, Perry C, Smith H, Filippich C, McLean D, McGrath J, Mackay-Sim A, Mowry B, Hayward NK (2008) Fibroblast and lymphoblast gene expression profiles in schizophrenia: are non-neural cells informative? PLoS One 3(6): e2412.

McCarthy MJ, Wei H, Marnoy Z, Darvish RM, McPhie DL, Cohen BM, Welsh DK (2013a) Genetic and clinical factors predict lithium's effects on PER2 gene expression rhythms in cells from bipolar disorder patients. Transl Psychiatry 3:e318.

McCarthy MJ, Fernandes M, Kranzler HR, Covault JM, Welsh DK (2013b) Circadian clock period inversely correlates with illness severity in cells from patients with alcohol use disorders. Alcohol Clin Exp Res 37(8):1304-1310.

McCarthy MJ, LeRoux M, Wei H, Beesley S, Kelsoe JR, Welsh DK (2015) Calcium channel genes associated with bipolar disorder modulate lithium's amplification of circadian rhythms. Neuropharmacology 101:439-448.

McGlashan TH (2011) Eugen Bleuler: centennial anniversary of his 1911 publication of Dementia Praecox or the group of schizophrenias. Schizophr Bull 37(6):1101-1103.

Meister A (1995) Glutathione biosynthesis and its inhibition. Methods Enzymol 252:26-30.

Mertens J, Paquola AC, Ku M, Hatch E, Böhnke L, Ladjevardi S, McGrath S, Campbell B, Lee H, Herdy JR, Gonçalves JT, Toda T, Kim Y, Winkler J, Yao J, Hetzer MW, Gage FH (2015) Directly reprogrammed human neurons retain aging-associated transcriptomic signatures and reveal age-related nucleocytoplasmic defects. Cell Stem Cell S1934-5909(15). pii:00408-7.

Mirnics K, Levitt P, Lewis DA (2006) Critical appraisal of DNA microarrays in psychiatric genomics. Biol Psychiatry 60(2): 163-176.

Moore NH, Costa LG, Shaffer SA, Goodlett DR, Guizzetti M (2009) Shotgun proteomics implicates extracellular matrix proteins and protease systems in neuronal development induced by astrocyte cholinergic stimulation. J Neurochem 108(4):891-908.

Moreira PI, Harris PL, Zhu X, Santos MS, Oliveira CR, Smith MA, Perry G (2007) Lipoic acid and N-acetyl cysteine decrease mitochondrial-related oxidative stress in Alzheimer disease patient fibroblasts. J Alzheimers Dis 12(2):195-206.

Mössner R, Daniel S, Albert D, Heils A, Okladnova O, Schmitt A, Lesch KP (2000) Serotonin transporter function is modulated by brain-derived neurotrophic factor (BDNF) but not nerve growth factor (NGF). Neurochem Int 36(3):197-202.

Mukherjee AB, Svoronos S, Ghazanfari A, Martin PR, Fisher A, Roecklein B, Rodbard D, Staton R, Behar D, Berg CJ (1987) Transketolase abnormality incultured fibroblasts from familial chronic alcoholic men and their maleoffspring. J Clin Invest 79 (4):1039-1043.

Müller N, Myint AM, Schwarz MJ (2012) Inflammation in schizophrenia. Adv Protein Chem Struct Biol 88:49-68.

Munzer A, Sack U, Mergl R, Schönherr J, Petersein C, Bartsch S, Kirkby KC, Bauer K, Himmerich H (2013) Impact of 
antidepressants on cytokine production of depressed patients in vitro. Toxins (Basel) 5(11):2227-2240.

Musliner KL, Seifuddin F, Judy JA, Pirooznia M, Goes FS, Zandi PP (2015) Polygenic risk, stressful life events and depressive symptoms in older adults: a polygenic score analysis. Psychol Med 45(8):1709-1720.

Naba A, Clauser KR, Ding H, Whittaker CA, Carr SA, Hynes RO (2015) The extracellular matrix: tools and insights for the "omics" era. Matrix Biol. pii: S0945-053X(15)00121-3.

Naderi J, Lopez C, Pandey S (2006) Chronically increased oxidative stress in fibroblasts from Alzheimer's disease patients causes early senescence and renders resistance to apoptosis by oxidative stress. Mech Ageing Dev 127(1):25-35.

Nadi NS, Nurnberger Jr JI, Gershon ES (1984) Muscarinic cholinergic receptors on skin fibroblasts in familial affective disorder. N Engl J Med 311(4):225-230.

Nagoshi E, Saini C, Bauer C, Laroche T, Naef F, Schibler U (2004) Circadian gene expression in individual fibroblasts: cellautonomous and self-sustained oscillators pass time to daughter cells. Cell 119(5):693-705.

Naydenov AV, MacDonald ML, Ongur D, Konradi C (2007) Differences in lymphocyte electron transport gene expression levels between subjects with bipolar disorder and normal controls in response to glucose deprivation stress. Arch Gen Psychiatry 64 (5):555-564.

Nestler EJ, Hyman SE (2010) Animal models of neuropsychiatric disorders. Nat Neurosci 13(10):1161-1169.

Nestor CE, Ottaviano R, Reinhardt D, Cruickshanks HA, Mjoseng HK, McPherson RC, Lentini A, Thomson JP, Dunican DS, Pennings S, Anderton SM, Benson M, Meehan RR (2015) Rapid reprogramming of epigenetic and transcriptional profiles in mammalian culture systems. Genome Biol 16(1):11.

Olsson E, Wiesel FA, Bjerkenstedt L, Venizelos N (2006) Tyrosine transport in fibroblasts from healthy volunteers and patients with schizophrenia. Neurosci Lett 393(2-3):211-215.

Ovadia G, Shifman S (2011) The genetic variation of RELN expression in schizophrenia and bipolar disorder. PLoS One 6 (5):e19955.

Pagani L, Semenova EA, Moriggi E, Revell VL, Hack LM, Lockley SW, Arendt J, Skene DJ, Meier F, Izakovic J, Wirz-Justice A, Cajochen C, Sergeeva OJ, Cheresiz SV, Danilenko KV, Eckert A, Brown SA (2010) The physiological period length of the human circadian clock in vivo is directly proportional to period in human fibroblasts. PLoS One 5(10):e13376.

Palazzo E, Marconi A, Truzzi F, Dallaglio K, Petrachi T, Humbert P, Schnebert S, Perrier E, Dumas M, Pincelli C (2012) Role of neurotrophins on dermal fibroblast survival and differentiation. J Cell Physiol 227(3):1017-1025.

Palotás A, Kálmán J, Palotás M, Juhász A, Janka Z, Penke B (2002) Fibroblasts and lymphocytes from Alzheimer patients are resistant to beta-amyloid-induced increase in the intracellular calcium concentration. Prog Neuropsychopharmacol Biol Psychiatry 26(5):971-974.

Pang ZP, Yang N, Vierbuchen T, Ostermeier A, Fuentes DR, Yang TQ, Citri A, Sebastiano V, Marro S, Südhof TC, Wernig M (2011) Induction of human neuronal cells by defined transcription factors. Nature 476(7359):220-223.

Park IH, Arora N, Huo H, Maherali N, Ahfeldt T, Shimamura A, Lensch MW, Cowan C, Hochedlinger K, Daley GQ (2008) Diseasespecific induced pluripotent stem cells. Cell 134(5):877-886.

Penninx BW, Milaneschi Y, Lamers F, Vogelzangs N (2013) Understanding the somatic consequences of depression: biological mechanisms and the role of depression symptom profile. BMC Med 11:129.

Persson ML, Johansson J, Vumma R, Raita J, Bjerkenstedt L, Wiesel FA, Venizelos N (2009) Aberrant amino acid transport in fibroblasts from patients with bipolar disorder. Neurosci Lett 457(1):49-52.

Peterson C, Goldman JE (1986) Alterations in calcium content and biochemical processes in cultured skin fibroblasts from aged and Alzheimer donors. Proc Natl Acad Sci U S A 83(8):2758-2762.
Przybyla LM, Theunissen TW, Jaenisch R, Voldman J (2013) Matrix remodeling maintains embryonic stem cell self-renewal by activating Stat3. Stem Cells 31(6):1097-1106.

Ramchand CN, Das I, Gliddon A, Hirsch SR (1994) Role of polyamines in the membrane pathology of schizophrenia. A study using fibroblasts from schizophrenic patients and normal controls. Schizophr Res 13(3):249-253.

Ramchand CN, Gliddon AE, Clark AE, Hemmings GP (1995) Glucose oxidation and monoamine oxidase activity from the fibroblasts of schizophrenic patients and controls. Life Sci 56 (19):1639-1646.

Ramchand CN, Peet M, Clark AE, Gliddon AE, Hemmings GP (1996) Decreased tyrosine transport in fibroblasts from schizophrenics: implications for membrane pathology. Prostaglandins Leukot Essent Fatty Acids 55(1-2):59-64.

Ranjekar PK, Hinge A, Hegde MV, Ghate M, Kale A, Sitasawad S, Wagh UV, Debsikdar VB, Mahadik SP (2003) Decreased antioxidant enzymes and membrane essential polyunsaturated fatty acids in schizophrenic and bipolar mood disorder patients. Psychiatry Res 121(2):109-122.

Reyazuddin M, Azmi SA, Islam N, Rizvi A (2014) Oxidative stress and level of antioxidant enzymes in drug-naive schizophrenics. Ind J Psychiatry 56(4):344-349.

Rieske P, Krynska B, Azizi SA (2005) Human fibroblast-derived cell lines have characteristics of embryonic stem cells and cells of neuro-ectodermal origin. Differentiation 73:474-483.

Rohani L, Johnson AA, Arnold A, Stolzing A (2014) The aging signature: a hallmark of induced pluripotent stem cells? Aging Cell 13(1):2-7.

Saini C, Brown SA, Dibner C (2015) Human peripheral clocks: applications for studying circadian phenotypes in physiology and pathophysiology. Front Neurol 6:95.

Sanacora G, Banasr M (2013) From pathophysiology to novel antidepressant drugs: glial contributions to the pathology and treatment of mood disorders. Biol Psychiatry 73(12): 1172-1179.

Santarelli S, Lesuis SL, Wang XD, Wagner KV, Hartmann J, Labermaier C, Scharf SH, Müller MB, Holsboer F, Schmidt MV (2014) Evidence supporting the match/mismatch hypothesis of psychiatric disorders. Eur Neuropsychopharmacol 24(6): 907-918.

Sarnyai Z, Alsaif M, Bahn S, Ernst A, Guest PC, Hradetzky E, Kluge W, Stelzhammer V, Wesseling H (2011) Behavioral and molecular biomarkers in translational animal models for neuropsychiatric disorders. Int Rev Neurobiol 101:203-238.

Schmidt MJ, Mirnics K (2012) Modeling interneuron dysfunction in schizophrenia. Dev Neurosci 34(2-3):152-158.

Schmidt MJ, Horvath S, Ebert P, Norris JL, Seeley EH, Brown J, Gellert L, Everheart M, Garbett KA, Grice TW, Caprioli RM, Mirnics K (2014) Modulation of behavioral networks by selective interneuronal inactivation. Mol Psychiatry 19(5):580-587.

Schmitt A, Parlapani E, Bauer M, Heinsen H, Falkai P (2008) Is brain banking of psychiatric cases valuable for neurobiological research? Clinics (Sao Paulo) 63(2):255-266.

Schmitt A, Malchow B, Hasan A, Falkai P (2014) The impact of environmental factors in severe psychiatric disorders. Front Neurosci 11(8):19.

Shahpasand K, Uemura I, Saito T, Asano T, Hata K, Shibata K, Toyoshima Y, Hasegawa M, Hisanaga S (2012) Regulation of mitochondrial transport and inter-microtubule spacing by tau phosphorylation at the sites hyperphosphorylated in Alzheimer's disease. J Neurosci 32(7):2430-2441.

Shelton RC, Mainer DH, Sulser F (1996) CAMP-dependent protein kinase activity in major depression. Am J Psychiatry 153(8): 1037-1042.

Shelton RC, Manier DH, Peterson CS, Ellis TC, Sulser F (1999) Cyclic AMP-dependent protein kinase in subtypes of major depression and normal volunteers. Int J Neuropsychopharmacol 2(3):187-192.

Shelton RC, Claiborne J, Sidoryk-Wegrzynowicz M, Reddy R, Aschner M, Lewis DA, Mirnics K (2011) Altered expression of 
genes involved in inflammation and apoptosis in frontal cortex in major depression. Mol Psychiatry 16(7):751-762.

Shivakumar V, Kalmady SV, Venkatasubramanian G, Ravi V, Gangadhar BN (2014) Do schizophrenia patients age early? Asian J Psychiatry 10:3-9.

Singh VK, Kalsan M, Kumar N, Saini A, Chandra R (2015) Induced pluripotent stem cells: applications in regenerative medicine, disease modeling, and drug discovery. Front Cell Dev Biol 3:2.

Smith EN, Bloss CS, Badner JA, Barrett T, Belmonte PL, Berrettini W, Byerley W, Coryell W, Craig D, Edenberg HJ, Eskin E, Foroud T, Gershon E, Greenwood TA, Hipolito M, Koller DL, Lawson WB, Liu C, Lohoff F, McInnis MG, McMahon FJ, Mirel DB, Murray SS, Nievergelt C, Nurnberger J, Nwulia EA, Paschall J, Potash JB, Rice J, Schulze TG, Scheftner W, Panganiban C, Zaitlen N, Zandi PP, Zöllner S, Schork NJ, Kelsoe JR (2009) Genome-wide association study of bipolar disorder in European American and African American individuals. Mol Psychiatry 14(8):755-763.

Soldner F, Laganière J, Cheng AW, Hockemeyer D, Gao Q, Alagappan R, Khurana V, Golbe LI, Myers RH, Lindquist S, Zhang L, Guschin D, Fong LK, Vu BJ, Meng X, Urnov FD, Rebar EJ, Gregory PD, Zhang HS, Jaenisch R (2011) Generation of isogenic pluripotent stem cells differing exclusively at two early onset Parkinson point mutations. Cell 146(2):318-331.

Song M, Mohamad O, Chen D, Yu SP (2013) Coordinated development of voltage-gated $\mathrm{Na}+$ and $\mathrm{K}+$ currents regulates functional maturation of forebrain neurons derived from human induced pluripotent stem cells. Stem Cells Dev 22(10): 1551-1563.

Sprenger A, Küttner V, Biniossek ML, Gretzmeier C, Boerries M, Mack C, Has C, Bruckner-Tuderman L, Dengjel J (2010) Comparative quantitation of proteome alterations induced by aging or immortalization in primary human fibroblasts and keratinocytes for clinical applications. Mol BioSyst 6(9):1579-1582.

Sugden K, Pariante CM, McGuffin P, Aitchison KJ, D'Souza UM (2010) Housekeeping gene expression is affected by antidepressant treatment in a mouse fibroblast cell line. $J$ Psychopharmacol 24(8):1253-1259.

Takahashi K, Tanabe K, Ohnuki M, Narita M, Ichisaka T, Tomoda K, Yamanaka S (2007) Induction of pluripotent stem cells from adult human fibroblasts by defined factors. Cell 131(5):861-872.

Tang S, Xie M, Cao N, Ding S (2015) Patient-specific induced pluripotent stem cells for disease modeling and phenotypic drug discovery. J Med Chem [Epub ahead of print].

Tatullo M, Marrelli M, Falisi G, Rastelli C, Palmieri F, Gargari M, Zavan B, Paduano F, Benagiano V (2015) Mechanical influence of tissue culture plates and extracellular matrix on mesenchymal stem cell behavior: a topical review. Int J Immunopathol Pharmacol. pii: 0394632015617951.

Terwisscha van Scheltinga AF, Bakker SC, Kahn RS, Kas MJ (2013) Fibroblast growth factors in neurodevelopment and psychopathology. Neuroscientist 19(5):479-494.

Tigges J, Krutmann J, Fritsche E, Haendeler J, Schaal H, Fischer JW, Kalfalah F, Reinke H, Reifenberger G, Stühler K, Ventura N, Gundermann S, Boukamp P, Boege F (2014) The hallmarks of fibroblast ageing. Mech Ageing Dev 138:26-44.

Toll A, Mané A (2015) Brain-derived neurotrophic factor levels in first episode of psychosis: A systematic review. World J Psychiatry 5(1):154-159.
Toouli CD, Huschtscha LI, Neumann AA, Noble JR, Colgin LM, Hukku B, Reddel RR (2002) Comparison of human mammary epithelial cells immortalized by simian virus $40 \mathrm{~T}$-Antigen or by the telomerase catalytic subunit. Oncogene 21(1):128-139.

Tosic M, Ott J, Barral S, Bovet P, Deppen P, Gheorghita F, Matthey ML, Parnas J, Preisig M, Saraga M, Solida A, Timm S, Wang AG, Werge T, Cuénod M, Do KQ (2006) Schizophrenia and oxidative stress: glutamate cysteine ligase modifier as a susceptibility gene. Am J Hum Genet 79(3):586-592.

Tourjman V, Kouassi É, Koué MĖ, Rocchetti M, Fortin-Fournier S, Fusar-Poli P, Potvin S (2013) Antipsychotics' effects on blood levels of cytokines in schizophrenia: a meta-analysis. Schizophr Res 151(1-3):43-47.

Vangipuram M, Ting D, Kim S, Diaz R, Schüle B (2013) Skin punch biopsy explant culture for derivation of primary human fibroblasts. $\mathrm{J}$ Vis Exp 77:e3779.

Waldera-Lupa DM, Kalfalah F, Florea AM, Sass S, Kruse F, Rieder V, Tigges J, Fritsche E, Krutmann J, Busch H, Boerries M, Meyer HE, Boege F, Theis F, Reifenberger G, Stühler K (2014) Proteome-wide analysis reveals an age-associated cellular phenotype of in situ aged human fibroblasts. Aging (Albany NY) 6(10):856-878.

Walker E, Mittal V, Tessner K (2008) Stress and the hypothalamic pituitary adrenal axis in the developmental course of schizophrenia. Annu Rev Clin Psychol 4:189-216.

Wang L, Lockstone HE, Guest PC, Levin Y, Palotás A, Pietsch S, Schwarz E, Rahmoune H, Harris LW, Ma D, Bahn S (2010) Expression profiling of fibroblasts identifies cell cycle abnormalities in schizophrenia. J Proteome Res 9(1):521-527.

Wassef AA, O'Boyle M, Gardner R, Rose RM, Brown A, Harris A, Nguyen H, Meyer 3rd WJ (1992) Glucocorticoid receptor binding in three different cell types in major depressive disorder: lack of evidence of receptor binding defect. Prog Neuropsychopharmacol Biol Psychiatry 16(1):65-78.

Wolkowitz OM, Epel ES, Reus VI, Mellon SH (2010) Depression gets old fast: do stress and depression accelerate cell aging? Depress Anxiety 27(4):327-338.

Yagita K, Tamanini F, van Der Horst GT, Okamura H (2001) Molecular mechanisms of the biological clock in cultured fibroblasts. Science 292(5515):278-281.

Yang S, Van Dongen HP, Wang K, Berrettini W, Bućan M (2009) Assessment of circadian function in fibroblasts of patients with bipolar disorder. Mol Psychiatry 14(2):143-155.

Yao JK, Leonard S, Reddy R (2006) Altered glutathione redox state in schizophrenia. Dis Markers 22(1-2):83-93.

Yoo AS, Sun AX, Li L, Shcheglovitov A, Portmann T, Li Y, LeeMesser C, Dolmetsch RE, Tsien RW, Crabtree GR (2011) MicroRNA-mediated conversion of human fibroblasts to neurons. Nature 476(7359):228-231.

Zeng H, Guo M, Martins-Taylor K, Wang X, Zhang Z, Park JW, Zhan S, Kronenberg MS, Lichtler A, Liu HX, Chen FP, Yue L, Li XJ, Xu $\mathrm{RH}$ (2010) Specification of region-specific neurons including forebrain glutamatergic neurons from human induced pluripotent stem cells. PLoS One 5(7):e11853.

Zhan L, Kerr JR, Lafuente MJ, Maclean A, Chibalina MV, Liu B, Burke B, Bevan S, Nasir J (2011) Altered expression and coregulation of dopamine signalling genes in schizophrenia and bipolar disorder. Neuropathol Appl Neurobiol 37(2):206-219. 


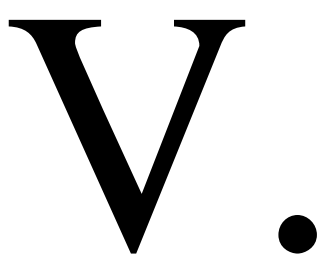




\title{
A Dishful of a Troubled Mind: Induced Pluripotent Stem Cells in Psychiatric Research
}

\author{
Sára Kálmán, ${ }^{1}$ Edit Hathy, ${ }^{2}$ and János M. Réthelyi ${ }^{2,3}$ \\ ${ }^{1}$ Department of Psychiatry, Faculty of Medicine, University of Szeged, Szeged, Hungary \\ ${ }^{2}$ National Brain Research Program, Hungarian Academy of Sciences, Molecular Psychiatry and \\ In Vitro Disease Modeling Research Group, Budapest, Hungary \\ ${ }^{3}$ Department of Psychiatry and Psychotherapy, Faculty of Medicine, Semmelweis University, Budapest, Hungary \\ Correspondence should be addressed to János M. Réthelyi; rethelyi.janos@med.semmelweis-univ.hu
}

Received 17 July 2015; Accepted 30 September 2015

Academic Editor: Xiaoyang Zhao

Copyright (c) 2016 Sára Kálmán et al. This is an open access article distributed under the Creative Commons Attribution License, which permits unrestricted use, distribution, and reproduction in any medium, provided the original work is properly cited.

\begin{abstract}
Neuronal differentiation of induced pluripotent stem cells and direct reprogramming represent powerful methods for modeling the development of neurons in vitro. Moreover, this approach is also a means for comparing various cellular phenotypes between cell lines originating from healthy and diseased individuals or isogenic cell lines engineered to differ at only one or a few genomic loci. Despite methodological constraints and initial skepticism regarding this approach, the field is expanding at a fast pace. The improvements include the development of new differentiation protocols resulting in selected neuronal populations (e.g., dopaminergic, GABAergic, hippocampal, and cortical), the widespread use of genome editing methods, and single-cell techniques. A major challenge awaiting in vitro disease modeling is the integration of clinical data in the models, by selection of well characterized clinical populations. Ideally, these models will also demonstrate how different diagnostic categories share overlapping molecular disease mechanisms, but also have unique characteristics. In this review we evaluate studies with regard to the described developments, to demonstrate how differentiation of induced pluripotent stem cells and direct reprogramming can contribute to psychiatry.
\end{abstract}

\section{Challenges in Psychiatric Research}

Schizophrenia (SCZ) and bipolar disorder (BPD) present with overlapping clinical symptomatology and share many environmental and genetic risk factors (for a review see [1]). Additionally, extended literature argues on the neurodevelopmental origin and neuroprogressive course of both syndromes. Although psychotic disorders and BPD are not the most frequent psychiatric conditions, they affected 8 million people in Europe and costed $€ 125$ billion for the society in 2010 according to the report of the European Brain Council [2].

As mental disorders are exclusively human conditions, investigating and modeling these conditions raise several problems and necessitate compromises. Animal models, based on rare mutations of large effects, provide valuable information on the cellular biology and behavioral endophenotypes of psychiatric disorders but obviously have their limitations and validation difficulties. Indeed, $92 \%$ of drugs that passed preclinical studies fail in the clinical phase due to lack of efficacy or safety reasons [3].

In vivo brain sampling in psychiatric patients or control, healthy subjects is ethically and technically problematic. Postmortem tissue samples are widely used for assessment of architectural and molecular alterations in brain disorders, but the results must be evaluated circumspectly regarding the variability in the sampled brain area, the pre- and postmortem circumstances and the consequent degradation of RNAs and proteins. In order to countervail these technical issues, brain banks provide great sample sizes, standardized methodology, and detailed clinical information; however, these samples are not appropriate for functional assays or diagnostic purposes and the observed changes might be evoked by comorbidities or environmental factors over the course of the disease. 
The heritability of SCZ, BPD, and autism spectrum disorders (ASD) is above $80 \%$ [4-6], but neither candidate gene nor genome-wide association (GWA) studies can fully explain this magnitude. These hidden genetics substantiated the theory of rare mutations with large effects versus common alleles with low penetrance [7]. Accordingly, in most of the cases psychiatric diseases are multifactorial and thus derive from the constellation of (otherwise harmless) common susceptibility alleles and environmental factors. Cases caused by single mutations occur very rarely and remain undetected in large-scale studies. Additionally, recent studies suggested that de novo mutations may have a great impact on the individual susceptibility $[8,9]$. In vitro cell culture models represent a system-oriented view, in which mental disorders are the manifestations of the donor's individual genetics, and along this line they enable performing functional assays to map gene $\times$ environment $(G \times E)$ and gene $\times$ gene $(G \times G)$ interactions.

\section{Manufacturing Neurons: Made in Dish}

Since detailed description of the iPSC/iNC induction and differentiation would extend the limitations of this paper and several publications have been already written on this rapidly developing field, here we will only briefly summarize the main technical issues (Figure 1). For further information and comparison of different protocols see [92-94].

Currently, there are three methods to generate human neural cells in vitro: iPSCs can be differentiated into neuronal progenitor cells (NPCs) and somatic cells can be transdifferentiated into neural stem cells or directly into neurons. Interestingly, transdifferentiation experiments have not yet been performed in the context of psychiatric disorders, even though four transcription factors or two microRNAs are enough to convert human fibroblasts into functional neurons within three weeks $[95,96]$. Direct conversion has the advantage of bypassing the prolonged, potentially mutagenic phases of reprogramming and intensive progenitor proliferation [97]. On the other hand, the amount of experimental material is limited by the number of somatic cells and the efficacy of the transdifferentiation.

Somatic cells can be reprogrammed into pluripotent stage with a set of transcriptional factors, namely, OCT4, SOX2, KLF4, and c-MYC or OCT4, SOX2, NANOG, and LIN28 $[98,99]$. These can be entered into the cells via integrating (lenti- and retroviral) or non-integrative (adenovirus, Sendai virus, episomal vector, and synthetic mRNA) vectors or direct protein delivering tools [100]. After initial induction, endogenous NANOG, SOX2, and OCT3/4 expression indicate pluripotency which can be maintained via basic fibroblast growth factor (bFGF) supplementation for theoretically unlimited time.

The differentiation of iPSCs is thought to follow in vivo developmental pathways and require environmental cues. During the past eight years several protocols have been developed based on monolayer dual SMAD inhibition [101] or embryoid aggregates [102] with an efficacy of $80 \%$ or more than $85 \%$, respectively. (For a comparative review see [103].) Successfully differentiated or transformed cells can be easily recognized by the detection of PAX6, an early forebrain neuronal marker. Since embryonic aggregate-based techniques reduce the variability of differentiation potential among pluripotent cells, it results in a more homogenous cell population. However, the culture always contains progenitors, glial cells, and mature or immature neurons with different neurotransmitter and receptor profiles and varying electrophysiological properties [13].

During manufacturing specific neurons, two major approaches are available. (1) High neurotransmitter specificity can be evoked by viral vectors or the combination of growth factors/small molecules. GABAergic cortical interneurons [104, 105], dopaminergic midbrain neurons $[106,107]$, dentate gyrus granule cells, and glutamatergic pyramidal neurons were successfully generated according to these protocols with an efficacy above 90\%. (2) On the other hand, one can address the investigation of region and layerspecific neurons. Remarkably, NPCs emerging from neural rosettes show self-organized spatiotemporal differentiation pattern and model the six-layered cortical structure. Benefitting from this, researchers are able to cultivate and isolate early and late cortical progenitors, preplate neurons, deep (VVI) and superficial (II-IV) layer neurons in a definite temporal manner [108]. Furthermore, advents of biotechnologies already allow us to grow neural and glial cells in 3D cultures, forming functional organoids which resemble certain brain regions on the cellular and tissue level [109]. These microphysiological systems could be especially valuable tools for modeling neurodevelopmental or neuroprogressive diseases.

The basic requirements against differentiation are as follows: quick and efficient generation of homogenous cell populations with physiological (or diseased) characteristics. The main concern about currently existing protocols is that they frequently result in heterogeneous, asynchronic cell populations with varying phenotypes and maturation states [13]. Remarkable efforts are made to develop cost-effective, largescale generation of NPCs and matured, synchronized neurons for high-throughput assays $[14,110]$.

\section{Closer to Perfection or Just Misperception?}

iPSC/iNC cultures as model systems have advantages and disadvantages: (1) the cell lines can be initiated from easily obtainable biospecimens, for example, blood sample, skin biopsy, hair follicle, or urine, (2) but the reprogramming, culturing, and differentiation are labor intensive and require notable expertise [111]. (3) iPSCs and iNPCs have selfsustaining capacity, (4) while differentiated neurons are in postmitotic state; hence they provide restricted experimental material. (5) During reprogramming and differentiation the cells undergo epigenetic rearrangement, (6) and proliferating iPSCs exhibit genetic instability which may result in population diversity and biases in genetic assays [112]. (7) Neurotransmitter and brain region specific neurons provide unique opportunity to study the pathophysiology and genetics of neuropsychiatric disorders, (8) but every new protocol has to be carefully validated regarding the cell type-specific markers and the analogies and discrepancies compared to in vivo and 


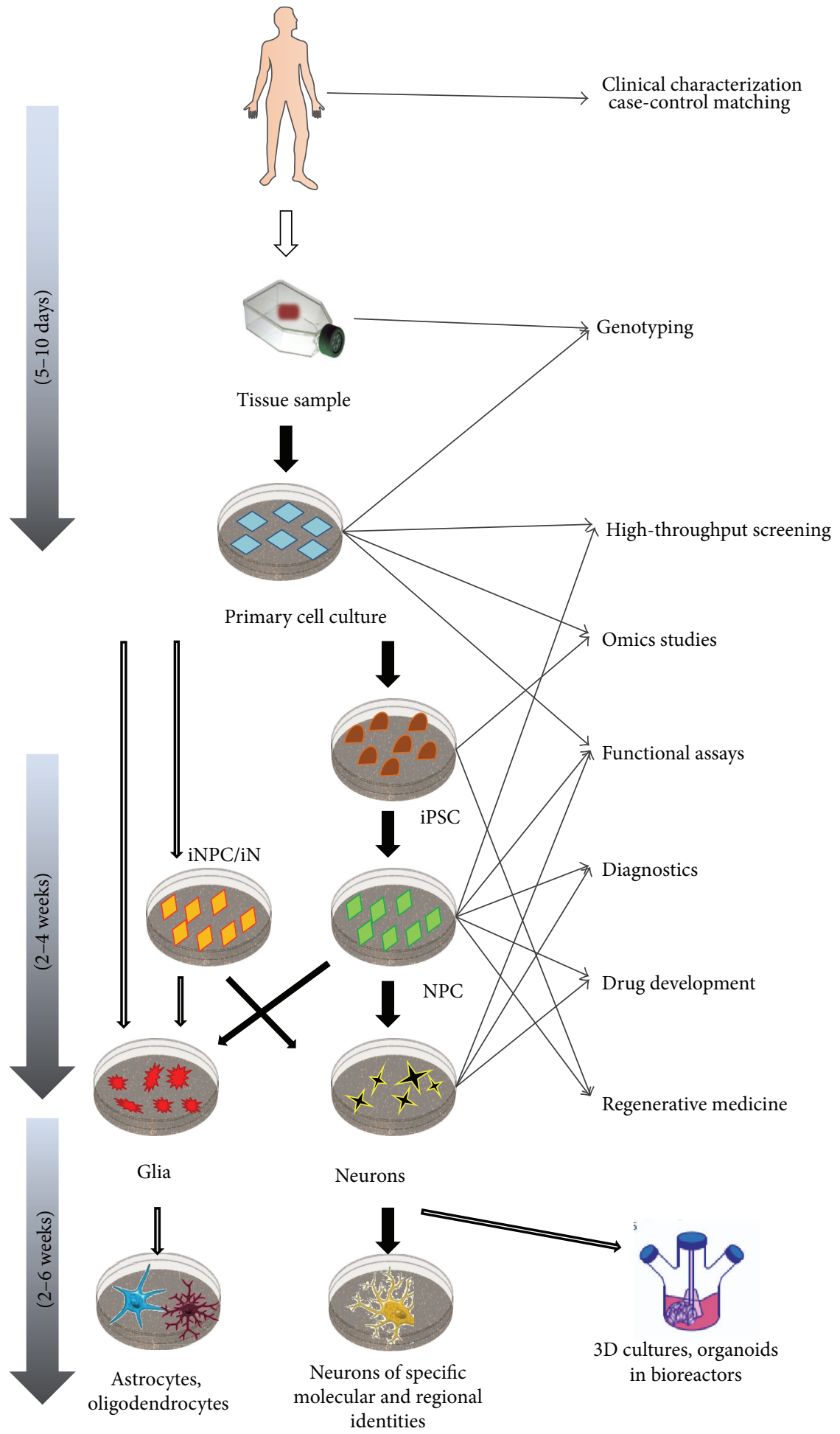

FIGURE 1: Schematic illustration of induced pluripotent stem cell and neural cell line generation and further clinical and research applications. (iPSC: induced pluripotent stem cell; iNPC: induced neural progenitor cell; iN: induced neuron). 


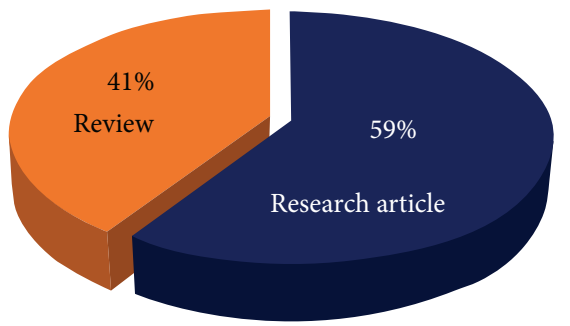

(a)

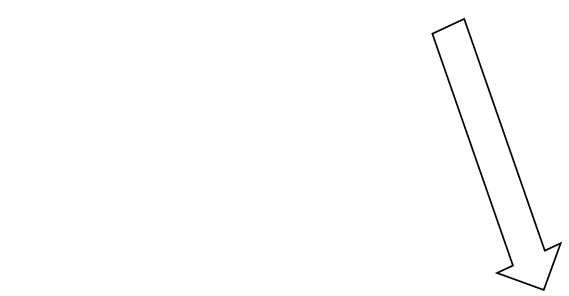

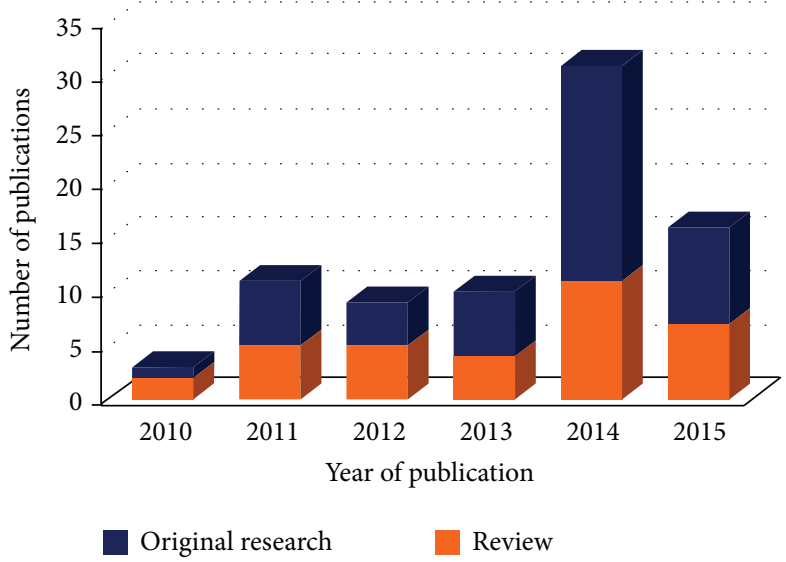

(b)

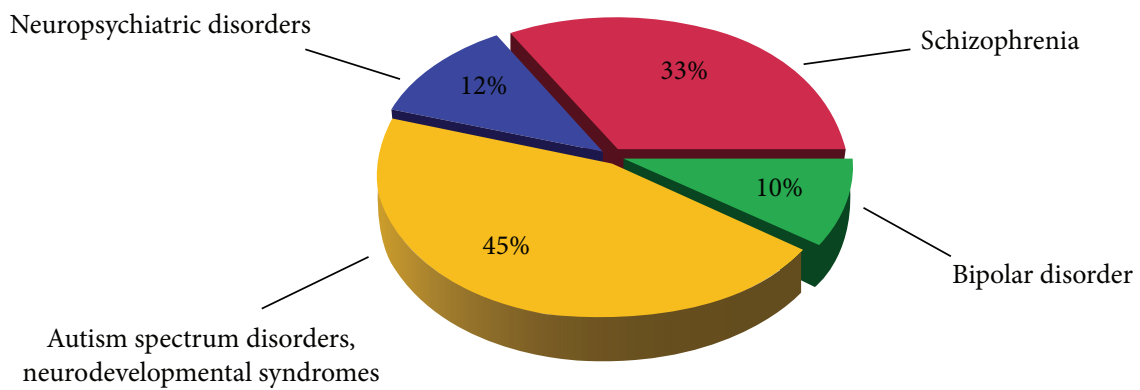

(c)

Figure 2: (a), (b), and (c) Characteristics of current literature dealing with induced pluripotent/neural cell lines in psychiatric research. (c) Represents the research articles only. Publications were reviewed until June, 2015.

animal model findings. (9) Finally, iPSC/iNC experiments are time and money consuming.

\section{Three Years' Balance: Debits and Credits}

In 2012, the National Institute of Mental Health (NIMH) and the Foundation for the NIH organized a workshop on the technological advances and challenges of patient-derived iPSCs/iNCs research in psychiatry. Scientists and delegates of industry and government and funding organizations conceived recommendations and directions for the future focusing on basic research, new target identification, and drug development (for the meeting report see [10]). Since stem cell biology and brain research are two of the most active fields of life sciences, we decided to review systematically the literature on iPSC/iNC in the context of psychiatric diseases and strike a balance: Did we take our own advice? How far have we got? What challenges do we face? (Table 1).

To give exact answers, we performed, first in the literature, a systematic review on iPSC/iNC based researches in psychiatry. We searched PubMed with the following keywords: "induced pluripotent OR reprogramming OR transdifferentiation AND psychiatry OR schizophrenia OR bipolar OR major depression OR autism" until 20 June, 2015. First, we catalogued research papers, if (at least part of) the experiment was conducted on human induced pluripotent derived or transdifferentiated neural cell cultures and English text was available. We excluded papers on neurodegenerative disorders, dementias, and epilepsies (Table 2(a)). During the survey, we noticed that review publications display outstanding proportion of the literature (41\%); thus we decided to list the relevant reviews using the same criteria (Table 2(b)).

\section{What Can (Should) Be Reviewed?}

After categorizing the items, we found 80 relevant publications: 48 research articles and 33 reviews (Figures 2(a), 2(b), and 2(c)). Intriguingly, we did not find any papers with iPSCs/iNCs targeting major depression. This is surprising, since unipolar depression is the leading chronic disorder in the WHO European Region, and the third cause of disability-adjusted life-years (DALY) [113], while only half of the patients receive adequate therapy with $70 \%$ longterm efficacy [114]. Considering that major depression is a multifactorial, neurobiological disorder with heritability estimates between 40 and 60\% [115] and peripheral cells were traditionally used to model and study the diseased endophenotypes [116-118], we can envisage that iPSCs/iNCs hold great promise in this field. 
TABLE 1: Advancements and further challenges of the utility of induced pluripotent/neural cells in psychiatric research. The recommendations were conceived on a meeting of the National Institute of Mental Health and the Foundation for NIH in 2012 [10].

\begin{tabular}{lll}
\hline Recommendations in 2012 & Advancements in the past 3 years & Challenges remain \\
\hline $\begin{array}{l}\text { (i) Optimizing reprogramming and } \\
\text { differentiating methods }\end{array}$ & \multicolumn{1}{c}{ Standardization of protocols } \\
$\begin{array}{l}\text { (ii) Efficient generation and validation of } \\
\text { specific neural cell types }\end{array}$ & $\begin{array}{l}\text { (i) Safe, integration-free, nonviral induction } \\
\text { (iii) The importance of region and }\end{array}$ & $\begin{array}{l}\text { protocols with efficacy }>80 \% \\
\text { (iii) Multiple model based studies }\end{array}$ \\
$\begin{array}{ll}\text { maturation state specific differentiation } \\
\text { (iv) Poorly defined regional identity }\end{array}$ & $\begin{array}{l}\text { (iv) Combination of GWAS databases with } \\
\text { iPSC/iNC observations }\end{array}$ & $\begin{array}{l}\text { (i) Comparison of cells induced from } \\
\text { different peripheral tissues }\end{array}$ \\
\end{tabular}

(i) Detailed comparison of inducedand

(i) Detailed comparison of induced and source cells to reveal de novo genetic mutations

(ii) Multiple parallel cell lines from one donor

(iii) Epigenetic mapping during reprogramming and differentiation

(i) Increasing sample sizes

(ii) Careful selection and grouping of subjects

(iii) Detailed clinical and genetic characterization of subjects (iv) Overthought diseased-control pairing

$$
\text { Improving homogeneity }
$$

(i) Vector integration-free, "safe" reprogramming methods

(ii) Reassuring results on chromosomal mutations

(iii) Average 3 cell lines/donor

(iv) Experiments and reviews comparing the available protocols (i) Concerns on de novo CNV mutations and the neuronal genome

(ii) Contradictions regarding epigenetics

(iii) Little is that known about endogenous production of astrocytes

$$
\text { Increasing statistical power }
$$

(i) Studies with whole genome sequencing and whole transcriptome profiling

(ii) Isogenic case-control comparison (new DNA editing techniques, twin studies) (i) Increasing sample sizes

(ii) Reconsideration of patient grouping

(iii) Transparent, published case-control matching

Improve reproducibility, resource sharing, and collaboration

(i) Establishing rigorous, transparent, and reproducible methods

(ii) Detailed publication of protocols (iii) Rapid sharing of cell lines, technologies, and best practices (iv) Improving public-private partnership

(i) Decreasing protocol diversity (ii) Validation assays for phenotypic comparison of derived cell lines

(i) Subgrouping on the base of comprehensive genetic and clinical characterization

(ii) Linking genotype with molecular and cellular pathophysiology (i) iPSC banks combined with gene banks

(ii) Commercially available iPSCs, iCell neurons, and knock-out cell lines with isogenic controls

(iii) Open access movements

(iv) Results usually correlated with postmortem and animal model findings Towards large-scale studies

(i) Protocol diversity remains, but major steps towards large-scale production

(ii) Commercially available cells provide enough experimental material for high throughput assays (i) Guidelines for validation

(ii) Criteria for cell characterization (markers, electrophysiological properties) (iii) Poor publication of donor's genotype, clinical features

\section{Careful patient selection, case-control matching}

(i) Isogenic case-control pairs provided by DNA editing techniques, twin studies

(ii) Pedigree-studies

(iii) DSM-5 reconsidered subcategories
Personalized medicine requires reprogramming and differentiation by every single patient, which is still remarkably time-consuming and money consuming

\section{Evidence-Based Questions}

iPSC/iNC studies are interpretable only in a multiscale disease modeling paradigm; thus results from a well-designed experiment should raise more questions than those they answer per se. Therefore, we examine the main issues addressed at the 2012 NIMH workshop [10] and those which emerged since via introducing some of the published studies and focusing on answered and emerged future challenges.

6.1. Time Is Not Everything, but Timing! The 2012 workshop highly emphasized the need for effective, standardized derivation and validation protocols for high-scale studies and future clinical application of iPSC/iNC technologies [10]. The participants cautioned against proliferation of poorly designed studies and recommended the establishment of rigorous, transparent, and reproducible methodology.

Reviewing the literature, we can conclude that significant progression was made in neural differentiation methods during the past three years. Today, researchers are able to generate neurons of regional and temporal identity, as well as 3D organoids; however, the methods are still under intense development for more efficient, time-sparing protocols. Since we have to allow room for these innovations, validation practices call for standardization. 
TABLE 2: (a) Research articles using induced pluripotent stem cells and/or induced neural cells in studying psychiatric disorders. (b) Review articles on induced pluripotent stem cells and/or induced neural cells in modeling psychiatric disorders.

(a)

\begin{tabular}{|c|c|c|c|c|c|}
\hline $\begin{array}{l}\text { Author, publication } \\
\text { date }\end{array}$ & Modeled disease(s) & Main findings & $\begin{array}{l}\text { Cell line, } \\
\text { differentiation protocol }\end{array}$ & $\begin{array}{l}\text { Patient } \\
\text { derived } \\
\text { cell lines }\end{array}$ & $\begin{array}{l}\text { Free } \\
\text { full text }\end{array}$ \\
\hline Ananiev et al., 2011 [11] & Rett syndrome & Neurons exhibit smaller nuclear size & $\begin{array}{c}\text { Differentiated } \\
\text { glutamatergic neurons }\end{array}$ & $\mathrm{Y}(3)$ & $\mathrm{Y}$ \\
\hline $\begin{array}{l}\text { Bavamian et al., } 2015 \\
\text { [12] }\end{array}$ & BPD & $\begin{array}{l}\text { miR-34a is associated with BPD and } \\
\text { neurodevelopment }\end{array}$ & NPCs & $\mathrm{Y}(1)$ & $\mathrm{N}$ \\
\hline Belinsky et al., 2014 [13] & Neurodevelopment & $\begin{array}{l}\text { Electrophysiology and gene expression } \\
\text { during neural maturation }\end{array}$ & $\begin{array}{c}\text { Differentiated } \\
\text { glutamatergic neurons }\end{array}$ & $\mathrm{Y}(1)$ & $\mathrm{Y}$ \\
\hline Boissart et al., 2013 [14] & $\begin{array}{l}\text { Psychopharmacology, } \\
\text { ASD }\end{array}$ & $\begin{array}{l}\text { Synchronous production of cortical } \\
\text { neurons for high-throughput assays }\end{array}$ & $\begin{array}{c}\text { Glutamatergic cortical } \\
\text { neurons }\end{array}$ & $\mathrm{Y}(2)$ & Y \\
\hline $\begin{array}{l}\text { Brennand et al., } 2011 \\
\text { [15] }\end{array}$ & SCZ & $\begin{array}{l}\text { Diminished connectivity, cAMP and } \\
\text { WNT signaling rescued by } \\
\text { antipsychotic treatment }\end{array}$ & $\begin{array}{c}\text { Panneuronal } \\
\text { differentiation } \\
\text { protocol, glutamatergic } \\
\text { neurons }\end{array}$ & $\mathrm{Y}(4)$ & $\mathrm{Y}$ \\
\hline $\begin{array}{l}\text { Brennand et al., } 2015 \\
{[16]}\end{array}$ & SCZ & $\begin{array}{l}\text { Altered migration, mitochondrial } \\
\text { damage, and increased oxidative stress }\end{array}$ & $\begin{array}{c}\text { NPCs, panneuronal } \\
\text { differentiation }\end{array}$ & $\mathrm{Y}(4)$ & $\mathrm{Y}$ \\
\hline Brick et al., 2014 [17] & ASD & $\begin{array}{l}\text { iPSC bank from ASD patients and } \\
\text { controls }\end{array}$ & $\begin{array}{c}\text { Differentiated } \\
\text { glutamatergic neurons }\end{array}$ & $\begin{array}{c}\mathrm{Y} \\
\text { (cell bank) }\end{array}$ & $\mathrm{Y}$ \\
\hline Bundo et al., 2014 [18] & SCZ & $\begin{array}{l}\text { LINE retroelements show more } \\
\text { activity in SCZ derived cells }\end{array}$ & $\begin{array}{c}\text { Differentiated } \\
\text { glutamatergic neurons }\end{array}$ & $\mathrm{Y}(3)$ & $\mathrm{Y}$ \\
\hline Chen et al., 2014 [19] & BPD & $\begin{array}{l}\text { BPD iNCs exhibit Ca-signaling and } \\
\text { neurodevelopment associated } \\
\text { transcription alterations }\end{array}$ & $\begin{array}{c}\text { Differentiated neurons } \\
\text { (mixed glutamatergic- } \\
\text { GABAergic } \\
\text { populations) }\end{array}$ & $\mathrm{Y}(3)$ & $\mathrm{Y}$ \\
\hline Chen et al., 2013 [20] & SCZ, BPD & $\begin{array}{l}\text { Transcriptional effects of zinc finger } \\
\text { protein } 804 \mathrm{~A} \text { silencing }\end{array}$ & $\begin{array}{c}\text { Differentiated } \\
\text { glutamatergic neurons }\end{array}$ & $\mathrm{N}$ & Y \\
\hline Cheung et al., 2011 [21] & Rett syndrome & $\begin{array}{l}\text { Generation of MECP } 2 \text { mutant } \\
\text { iPSC/iNC lines and their isogenic pairs }\end{array}$ & $\begin{array}{c}\text { Differentiated } \\
\text { glutamatergic neurons }\end{array}$ & $\mathrm{Y}(1)$ & $\mathrm{Y}$ \\
\hline Chiang et al., 2011 [22] & SCZ & $\begin{array}{l}\text { Introduction of an integration-free } \\
\text { method for reprogramming }\end{array}$ & iPSCs & $\mathrm{Y}(2)$ & $\mathrm{Y}$ \\
\hline $\begin{array}{l}\text { Corrales et al., } 2012 \\
\text { [23] }\end{array}$ & SCZ & $\begin{array}{l}\text { Copy number variations in iPSCs, } \\
\text { iNCs }\end{array}$ & NPCs & $\mathrm{Y}^{*}$ & $\mathrm{~N}$ \\
\hline Dage et al., 2014 [24] & $\begin{array}{l}\text { Psychopharmacology, } \\
\text { ASD }\end{array}$ & $\begin{array}{l}\text { Pharmacological and transcriptome } \\
\text { characterization of iNCs }\end{array}$ & Forebrain neurons & $\mathrm{N}$ & $\mathrm{N}$ \\
\hline DeRosa et al., 2012 [25] & ASD & $\begin{array}{l}\text { iPSC and GABA neuron derivation } \\
\text { from whole blood }\end{array}$ & GABAergic neurons & $\mathrm{Y}^{*}$ & $\mathrm{Y}$ \\
\hline Doers et al., 2014 [26] & Fragile X syndrome & iNCs show neurite outgrowth deficit & Forebrain neurons & $\mathrm{Y}(3)$ & $\mathrm{N}$ \\
\hline $\begin{array}{l}\text { Germain et al., } 2014 \\
\text { [27] }\end{array}$ & $\begin{array}{c}\text { Neurodevelopmental } \\
\text { disorders }\end{array}$ & $\begin{array}{l}\text { Gene expression analysis of iPSCs } \\
\text { from } 15 \text { q11 variants }\end{array}$ & $\begin{array}{c}\text { Differentiated } \\
\text { glutamatergic neurons }\end{array}$ & $\mathrm{Y}(3)$ & $\mathrm{Y}$ \\
\hline $\begin{array}{l}\text { Griesi-Oliveira et al., } \\
2014 \text { [28] }\end{array}$ & ASD & TRPC6 gene is associated with ASD & $\begin{array}{c}\text { Differentiated } \\
\text { glutamatergic neurons }\end{array}$ & $\mathrm{Y}(1)$ & $\mathrm{N}$ \\
\hline $\begin{array}{l}\text { Hashimoto-Torii et al., } \\
2014 \text { [29] }\end{array}$ & SCZ & $\begin{array}{l}\text { Heat shock transcription factor } 1 \\
\text { mediated stress response abnormalities } \\
\text { in a subpopulation of iNPCs }\end{array}$ & NPCs & $\mathrm{Y}(4)$ & $\mathrm{Y}$ \\
\hline Hook et al., 2014 [30] & SCZ & $\begin{array}{l}\text { Increased catecholaminerg neural } \\
\text { activity in SCZ cell cultures }\end{array}$ & $\begin{array}{c}\text { Panneuronal } \\
\text { differentiation protocol }\end{array}$ & $\mathrm{Y}(4)$ & $\mathrm{Y}$ \\
\hline Chung et al., 2014 [31] & Fragile X syndrome & $\begin{array}{l}\text { Development of a high-content } \\
\text { screening assay }\end{array}$ & NPCs & $\mathrm{Y}^{*}$ & $\mathrm{~N}$ \\
\hline Krey et al., 2013 [32] & Timothy syndrome & $\begin{array}{l}\text { iNCs exhibit dendritic retraction } \\
\text { deficit }\end{array}$ & $\begin{array}{l}\text { Differentiated } \\
\text { glutamatergic neurons }\end{array}$ & $\mathrm{Y}(2)$ & $\mathrm{Y}$ \\
\hline
\end{tabular}


(a) Continued.

\begin{tabular}{|c|c|c|c|c|c|}
\hline $\begin{array}{l}\text { Author, publication } \\
\text { date }\end{array}$ & Modeled disease(s) & Main findings & $\begin{array}{l}\text { Cell line, } \\
\text { differentiation protocol }\end{array}$ & $\begin{array}{l}\text { Patient } \\
\text { derived } \\
\text { cell lines }\end{array}$ & $\begin{array}{l}\text { Free } \\
\text { full text }\end{array}$ \\
\hline Kumari et al., 2015 [33] & Fragile X syndrome & $\begin{array}{l}\text { Development of a high-throughput } \\
\text { screening assay }\end{array}$ & NPCs & $\mathrm{Y}(3)$ & $\mathrm{N}$ \\
\hline $\begin{array}{l}\text { Larimore et al., } 2013 \\
{[34]}\end{array}$ & Rett syndrome & $\begin{array}{l}\text { MECP2 regulates synaptic expression } \\
\text { of dysbindin-BLOC1 pathway }\end{array}$ & $\begin{array}{c}\text { Differentiated } \\
\text { glutamatergic neurons }\end{array}$ & $\mathrm{Y}(2)$ & $\mathrm{Y}$ \\
\hline Lin et al., 2012 [35] & SCZ & Allele specific expression profile & $\begin{array}{c}\text { Differentiated } \\
\text { glutamatergic neurons }\end{array}$ & Y (3) & $\mathrm{Y}$ \\
\hline Lin et al., 2014 [36] & SCZ, ASD & $\begin{array}{l}\text { Heat shock alters SCZ, ASD-related } \\
\text { genes }\end{array}$ & $\begin{array}{c}\text { 3-dimensional } \\
\text { neuronal aggregates }\end{array}$ & $\mathrm{Y}$ & $\mathrm{Y}$ \\
\hline Liu et al., 2012 [37] & Fragile X syndrome & $\begin{array}{l}\text { FMR1 mutation linked phenotype and } \\
\text { signaling deficits }\end{array}$ & Differentiated neurons & $\begin{array}{c}\text { Risk } \\
\text { variant } \\
\text { carrier }\end{array}$ & $\mathrm{Y}$ \\
\hline $\begin{array}{l}\text { Madison et al., } 2015 \\
\text { [38] }\end{array}$ & BPD & $\begin{array}{l}\text { Phenotypic alterations in BPD } \\
\text { progenitors rescued by WNT } \\
\text { inhibition }\end{array}$ & NPCs & $\begin{array}{l}\mathrm{Y}(2) \\
\text { (pedigree- } \\
\text { study) }\end{array}$ & $\mathrm{N}$ \\
\hline $\begin{array}{l}\text { Maekawa et al., } 2015 \\
\text { [39] }\end{array}$ & SCZ, ASD & $\begin{array}{l}\text { Hair follicle is a potential biomarker } \\
\text { source }\end{array}$ & $\begin{array}{l}\text { iPSC-derived } \\
\text { neurospheres }\end{array}$ & Y & $\mathrm{Y}$ \\
\hline $\begin{array}{l}\text { Marchetto et al., } 2010 \\
{[40]}\end{array}$ & Rett syndrome & $\begin{array}{l}\text { Morphological and } \\
\text { electrophysiological anomalies }\end{array}$ & $\begin{array}{c}\text { Panneuronal } \\
\text { differentiation protocol }\end{array}$ & Y & $\mathrm{Y}$ \\
\hline $\begin{array}{l}\text { Maschietto et al., } 2015 \\
\text { [41] }\end{array}$ & SCZ & $\begin{array}{l}\text { Altered gene expression profile during } \\
\text { neurodevelopment }\end{array}$ & NPCs & Y (1) & $\mathrm{Y}$ \\
\hline $\begin{array}{l}\text { Niedringhaus et al., } \\
2015 \text { [42] }\end{array}$ & Fragile X syndrome & $\begin{array}{l}\text { Mobile raft minicultures developed for } \\
\text { high-throughput assays on neurons }\end{array}$ & $\begin{array}{l}\text { Differentiated neurons } \\
\text { in microraft cultures }\end{array}$ & Y (1) & $\mathrm{Y}$ \\
\hline Paşca et al., 2011 [43] & Timothy syndrome & $\begin{array}{l}\text { Disease-specific cellular phenotype } \\
\text { and differentiation }\end{array}$ & $\begin{array}{c}\text { Cortical glutamatergic } \\
\text { neurons } \\
\end{array}$ & $\mathrm{Y}(2)$ & $\mathrm{Y}$ \\
\hline Paulsen et al., 2014 [44] & SCZ & $\begin{array}{l}\text { Zinc and potassium imbalance } \\
\text { reverted by valproate }\end{array}$ & NPCs & $\mathrm{Y}(2)$ & $\mathrm{N}$ \\
\hline Pedrosa et al., 2011 [45] & SCZ & 22q11.2 deletion delays differentiation & Glutamatergic neurons & $\mathrm{Y}(3)$ & $\mathrm{N}$ \\
\hline $\begin{array}{l}\text { Robicsek et al., } 2013 \\
{[46]}\end{array}$ & SCZ & $\begin{array}{l}\text { Impaired differentiation, maturation, } \\
\text { and mitochondrial dysfunction }\end{array}$ & Dopaminergic neurons & Y (3) & $\mathrm{N}$ \\
\hline Roussos et al., 2014 [47] & SCZ & $\begin{array}{l}\text { CACNA1C variation disrupts gene } \\
\text { regulation through chromosome loops }\end{array}$ & $\begin{array}{c}\text { Differentiated } \\
\text { glutamatergic neurons }\end{array}$ & $\mathrm{N}$ & $\mathrm{Y}$ \\
\hline $\begin{array}{l}\text { Shcheglovitov et al., } \\
2013 \text { [48] }\end{array}$ & $\begin{array}{l}\text { 22q13.3 deletion } \\
\text { syndrome }\end{array}$ & $\begin{array}{l}\text { SHANK3 and IGF1 correct excitatory } \\
\text { synaptic transmission deficit }\end{array}$ & $\begin{array}{c}\text { Differentiated } \\
\text { glutamatergic neurons }\end{array}$ & $\mathrm{Y}(2)$ & $\mathrm{N}$ \\
\hline $\begin{array}{l}\text { Sheridan et al., } 2011 \\
\text { [49] }\end{array}$ & Fragile X syndrome & Diminished neural differentiation & $\begin{array}{l}\text { Differentiated neurons } \\
\text { and glia }\end{array}$ & $\mathrm{Y}(3)$ & $\mathrm{Y}$ \\
\hline Shi et al., 2014 [50] & Psychopharmacology & $\begin{array}{l}\text { Dopamine } 2 \text { receptor is mediated by } \\
\text { microRNA- } 9 \text { and microRNA- } 326\end{array}$ & Dopaminergic neurons & $\mathrm{N}$ & $\mathrm{Y}$ \\
\hline Tian et al., 2014 [51] & Timothy syndrome & $\begin{array}{l}\text { Altered } \mathrm{Ca}^{2+} \text { signaling leads to } \\
\text { transcriptional dysregulation }\end{array}$ & $\begin{array}{c}\text { Differentiated } \\
\text { glutamatergic neurons }\end{array}$ & Y (3) & $\mathrm{Y}$ \\
\hline Topol et al., 2015 [52] & SCZ & Altered WNT signaling & $\begin{array}{l}\text { Forebrain patterned } \\
\text { NPCs } \\
\end{array}$ & $\mathrm{Y}(4)$ & $\mathrm{N}$ \\
\hline Wang et al., 2014 [53] & BPD & $\begin{array}{l}\text { Cell adhesiveness is associated with } \\
\text { lithium response }\end{array}$ & $\begin{array}{l}\text { Immature iNs, } \\
\text { lentiviral-based } \\
\text { transdifferentiation }\end{array}$ & $\mathrm{Y}(12)$ & $\mathrm{Y}$ \\
\hline Wen et al., 2014 [54] & SCZ, MDD & $\begin{array}{l}\text { DISC1 mutation causes synaptic } \\
\text { deficits and transcription } \\
\text { dysregulation }\end{array}$ & $\begin{array}{l}\text { Glutamatergic } \\
\text { forebrain neurons }\end{array}$ & $\mathrm{Y}(2)$ & $\mathrm{N}$ \\
\hline $\begin{array}{l}\text { Williams et al., } 2014 \\
\text { [55] }\end{array}$ & Rett syndrome & $\begin{array}{l}\text { MECP2 mutant astrocyte influences } \\
\text { negatively the morphology and } \\
\text { function of cocultured neurons }\end{array}$ & Astrocytes & $\mathrm{Y}$ & $\mathrm{Y}$ \\
\hline
\end{tabular}


(a) Continued.

\begin{tabular}{lclccc}
\hline $\begin{array}{l}\text { Author, publication } \\
\text { date }\end{array}$ & Modeled disease(s) & Main findings & $\begin{array}{c}\text { Cell line, } \\
\text { differentiation protocol }\end{array}$ & $\begin{array}{c}\text { Patient } \\
\text { derived } \\
\text { cell lines }\end{array}$ & $\begin{array}{c}\text { Free } \\
\text { full text }\end{array}$ \\
\hline Yoon et al., 2014 [56] & SCZ & $\begin{array}{l}15 q 11.2 \text { CNV results in neural stem cell } \\
\text { deficit }\end{array}$ & Neural rosettes, NPCs & Y & Y \\
\hline Yu et al., 2014 [57] & SCZ & $\begin{array}{l}\text { Deficit in hippocampal granule neuron } \\
\text { generation }\end{array}$ & $\begin{array}{c}\text { Hippocampus dentate } \\
\text { gyrus granule cells }\end{array}$ & Y (4) & Y \\
\hline Zeng et al., 2013 [58] & Neurodevelopment & $\begin{array}{l}\text { NRXN1 silencing impacts adhesion } \\
\text { and differentiation related } \\
\text { transcription }\end{array}$ & NPCs and & N & Y \\
\hline
\end{tabular}

* indicates data were not available.

ASD: autism spectrum disorders; BPD: bipolar disorder; iPSC: induced pluripotent stem cell; MDD: major depressive disorder; SCZ: schizophrenia; NPC: neural progenitor cell.

(b)

\begin{tabular}{|c|c|c|}
\hline Author(s) (31) & Year of publication & Disease(s) \\
\hline Acab and Muotri & $2015[59]$ & ASD \\
\hline Aigner et al. & $2014[60]$ & ASD \\
\hline Ardhanareeswaran et al. & $2015[61]$ & ASD \\
\hline Brennand and Gage & $2012[62]$ & Psychiatric disorders \\
\hline Brennand et al. & $2014[63]$ & SCZ \\
\hline Buxbaum and Sklar & $2011[64]$ & SCZ \\
\hline Chailangkarn et al. & $2012[65]$ & Neurodevelopmental disorders \\
\hline Cheung et al. & $2012[66]$ & Rett syndrome \\
\hline Cundiff and Anderson & $2011[67]$ & Neuropsychiatric disorders \\
\hline Duan & $2015[68]$ & SCZ \\
\hline Freitas et al. & 2014 [69] & ASD \\
\hline Ho et al. & $2015[70]$ & Neuropsychiatric disorders \\
\hline Cocks et al. & $2014[71]$ & ASD \\
\hline Kim & $2010[72]$ & Psychiatric disorders \\
\hline Kim et al. & $2012[73]$ & ASD \\
\hline Kim et al. & $2014[74]$ & ASD \\
\hline Ladran et al. & $2013[75]$ & Neuropsychiatric disorders \\
\hline Liu and Scott & $2014[76]$ & ASD \\
\hline Mackay-Sim et al. & $2011[77]$ & Neuropsychiatric disorders \\
\hline Muotri & $2015[78]$ & ASD \\
\hline O'Shea and McInnis & 2015 [79] & BPD \\
\hline Paşca et al. & $2014[80]$ & Neuropsychiatric disorders \\
\hline Paulsen et al. & $2012[81]$ & SCZ \\
\hline Paulsen et al. & $2013[82]$ & Neurodevelopmental disorders \\
\hline Prilutsky et al. & $2014[83]$ & ASD \\
\hline Qiang et al. & $2014[84]$ & Neuropsychiatric disorders \\
\hline Schadt et al. & 2014 [85] & Neuropsychiatric disorders \\
\hline Tobe et al. & $2013[86]$ & Psychopharmacology \\
\hline Tran et al. & 2013 [87] & SCZ \\
\hline Vaccarino et al. & $2011[88]$ & Neuropsychiatric disorders \\
\hline Viswanath et al. & 2015 [89] & BPD \\
\hline Walsh and Hochedlinger & $2010[90]$ & Rett syndrome \\
\hline Wright et al. & 2014 [91] & SCZ \\
\hline
\end{tabular}

ASD: autism spectrum disorders; BPD: bipolar disorder; SCZ: schizophrenia. 
Key questions are as follows: What are we modeling? (Which developmental and functional state of the in vivo neurons?) What kind of indicators and assays should be used for quality control? iPSCs are considered almost undistinguishable compared to human embryonic stem cells; and iPSC derived NPCs form rosettes, analog to the neural tube, the embryonic primordium of the central nervous system [119]. Thus, it is reasonable to assume that we are modeling fetal neurogenesis and neurodevelopment in cell cultures under differentiation. Accordingly, Brennand et al. found that the gene expression profile of NPCs and even 6-weekold differentiated neurons resembles the first-trimester fetal brain at the most [16]. Moreover, the electrophysiological properties of stem cell derived neurons share common temporal pattern with pyramidal neurons of the postnatal animal neocortex [120].

However, we have to declare that our knowledge on preand postnatal brain development is restricted and mainly relies on animal and human fetal brain studies. This limited insight and the in vitro observations underpin that iPSC differentiation and iNC maturing follow the in vivo timeline and stages and react to the same exogenous effects [93].

For instance, Boissart et al. demonstrated that the differentiational potential of iNPCs follows a temporal manner similar to what has been previously described in animal models [14]. The group delayed cellular commitment with high-mitogenic medium and sustained NPC proliferation for passages $8-20$. The prolonged proliferation period resulted in a homogeneous late cortical progenitor population that spontaneously differentiated into superficial cortical neurons. Three weeks after withdrawal of mitogenic factors, more than $80 \%$ of the cells were glutamatergic. Importantly, the short, synchronous, and highly productive differentiation period makes this method amenable to high-throughput assays.

6.2. Doing Well in the Wells. Neurons, specified from progenitor cells, are immature and require 4-12 weeks to reach their definitive phenotype in vitro, which $[119,121,122]$ is a notable hampering factor of iNC studies. Literature proves that detailed investigation of this period is crucial for the following technical and validation reasons.

(1) First, transition from pluripotency to differentiated neuronal state is attended by complex, pervasive gene expression changes. Fathi et al. analyzed the differentiation-related transcriptome dynamics and revealed that 5955 transcripts were modified during the 4-week-long protocol [123]. Of note, 2589 transcripts were upregulated in the differentiated neurons compared to pluripotent cells. On one hand, this intensive, early period can be used to unveil and understand neurodevelopmental disturbances and bridge between disease-associated genotypes and endophenotypes. On the other hand, experimental design, timing, and theories must be set up circumspectly.

(2) Differentiation and maturation can be influenced by the culturing conditions; therefore, several researches proposed protocol modifications to shorten the "before-theexperiment" period and improve synchronicity. For instance, Tang et al. compared the neural maturation on two different surfaces: the most frequently used artificial coating, laminin, versus astrocyte layer [124]. Astrocytes promoted the differentiation, soma and neurite growth, and dendrite arborization. They supported functional maturation with regard to ion channel and receptor expression and synaptic transmission. Still, even if cultured on astrocytes, neurons did not exhibit matured synaptic activity before the third week, and cells on laminin were further delayed. Astrocyte-conditioned [125], ascorbic acid [126] and cAMP supplemented [123] culturing medium or the application of graphene oxide nanomaterial [127] also accelerates differentiation.

(3) We also cannot pass by the fact that the extended, responsive maturation may lead to phenotypic heterogeneity within the dish and result in a mix of progenitors and immature, partially or fully matured neurons in different ratios [13]. Additionally, NPCs and neurons display similar appearance and NPCs express glutamate [128], GABA [129], and dopamine $[130]$ receptors.

6.3. Casting Neurons. Given the above-mentioned issues, we suggest that every iPSC/iNC experiment should include the developmental and functional characterization of the subject cells. The main approaches for describing a neuron are as follows: neural marker detection, receptor and ion channel profiling, electrophysiological analysis, and the evaluation of synaptic functions via enzyme activity, neurotransmitter release, metabolism, and reuptake [131].

The methodology of neural marker detection developed concomitantly with the differentiation protocols. Today, we are able to generate and identify cortical excitatory glutamatergic pyramidal cells [102]; GABAergic inhibitory interneurons [105]; cerebellar Purkinje cells [122]; or dopaminergic neurons of the substantia nigra [131]. However, the presence of the differentiation markers is not indicative of the neuron's maturation state.

The work of Dage et al. revealed that detailed pharmacological characterization of the differentiated neurons would be much desired since the receptor and ion channel signature of differentiated neurons may change almost day by day [24]. Furthermore, maturing iPSC-derived neurons do not display NMDA receptor subunit switch, peculiar to the neonatal brain [24], which might be a relevant difference between in vitro and in vivo fashions.

Data suggest that electrophysiological assessment of the cells might be the most potent approach for defining maturation states. The electrophysiological development in vitro resembles postnatal neocortical changes observed in animal models. Namely, the resting membrane potential becomes more negative, the duration of action potential decreases, and the $\mathrm{Na}^{+}, \mathrm{K}^{+}$, and $\mathrm{Ca}^{2+}$ currents show timedependent changes [120]. The milestones are thought to be the appearance of spontaneous excitatory postsynaptic currents (sEPSC) and action potentials in fresh neurons [122] and capability for repetitive action potentials in matured neurons $[13,120,131]$.

Belinsky et al. went further. They carried out patch clamp, $\mathrm{Ca}^{2+}$ imaging, and PCR on single-cell level to correlate the electrophysiological properties and the gene expression pattern of differentiating and maturing neurons [13]. The 
cells demonstrated action potential already from day 15, but expression of several neuronal physiology and diseaseassociated genes were delayed until day 29 (COMT, DISC1, $D T N B P 1, G A D 1$, and $P A X 6)$. These thought-provoking results press for multimethod validation. Recently, Chatzidaki et al. [132] demonstrated the detailed pharmacological characterization of nicotinic acetylcholine receptors on iPSC-derived human neurons. The exact time points of the functional and developmental stages highly depend on the cell type and the culturing protocol; therefore, an orientating timeline with the defined, critical landmarks and minimally required validation assays could assist the interpretation and reproducibility of in vitro findings. Furtherly, high-throughput assays offer the possibility to monitor the cell cultures around the clock, analyze structural and functional alterations (e.g., synaptic activity), and capture the right time to run hundreds of tests.

6.4. Neurons, into Single File! We are only beginning to realize the magnitude of heterogeneity in neuronal cultures derived from iPSCs. Earlier lines of evidence already highlighted the fact that, despite the utilization of targeted differentiation protocols, neuronal cultures remain heterogeneous and give rise to mixed populations of glutamatergic, GABAergic, and dopaminergic neurons, as well as astrocytes and undifferentiated cells. Recently, the development of single-cell approaches makes it possible to determine the ratio of neurons versus astroglia, neuronal subpopulations, or neurons reaching a specific stage of maturation, within a population of differentiating neurons. Neuron-to-neuron variation seems to be a general feature of in vitro as well as in vivo neuronal populations. Moreover the degree of variation also casts light on faulty neurodevelopment associated with neuropsychiatric disorders. Shcheglovitov et al. [48] used singlecell methodology to analyze neuronal cultures derived from patients suffering from Phelan-McDermid syndrome, a rare condition caused by 22q13.3 deletion. Besides other major findings, this outstanding paper also points out that only a small proportion of neurons express postsynaptic density proteins SHANK1-SHANK3, indicating small fraction of synaptically mature neurons. 20-60\% of neurons expressed TBR1, CTIP2, and SATB2 upper layer cortical markers, while less than $10 \%$ of neurons expressed GABAergic markers. In their methodological summary, Citri et al. [133] provide a protocol for single-iN qPCR and present data indicating the low proportion of VGLUT1 and VGLUT2 expressing iNs, suggesting delayed synaptic maturation in this system [134]. The described single-cell methodology has been successfully incorporated in several other studies. While neuronal differentiation protocols are much better characterized in recent years, the above findings illustrate that single-cell approaches are on the rise and will be important and necessary tools in the armamentarium of in vitro neuronal disease modeling efforts.

6.5. Thinking Big. Theoretical concepts of psychiatric disorders changed radically in the recent decades: the immuneneurodevelopmental model of SCZ and ASD [135], neuroinflammation-degeneration theory of MDD [136], and the need for $\mathrm{G} \times \mathrm{E}$ and network-based diagnostic and therapeutic approaches became widely accepted. But the revolution is still delayed in psychopharmacology. One detrimental factor can be the lengthy, expensive, and animal model based testing of potential new targets and compounds. Therefore, robotic high-throughput assays on human cell lines could be milestones in the paradigm shift towards human biology based, in vitro drug development [137]. iPSC/iNCs can be optimal subjects for these studies.

Fragile $\mathrm{X}$ syndrome is neurodevelopmental disorder caused by a silencing mutation of the FMR1 gene. Recently, two independent research groups developed and published high-throughput screening methods for FMR protein detection and novel drug identification using patient-derived iNPCs $[33,138]$. Kumari et al. designed a time-resolved fluorescence energy transfer (TR-FRET) based assay to measure FMRP levels in 1536 wells and screen 1280 pharmacologically active compounds parallel to identify those which increase the expression of the silenced gene [33]. The most effective molecules were retested in a secondary assay using qRTPCR and further confirmed by dose-response experiment. Kaufmann et al. used high-content screening to observe cell morphology and FMRP expression alterations and tested the efficacy and toxicity of 50000 compounds [138]. Importantly, automated high resolution microscopy and machine learning algorithms allow single-cell-based follow-up and data analysis in living cultures; therefore the group was able to detect that a remarkable subpopulation of the cells (40\%) responds to certain drugs, although the averaged measures did not reach significance.

Both works demonstrated a sensitive, cost-effective approach for drug development. However, we have to add that the utility of progenitor cells is frequently suboptimal or invalid in psychopharmacology; and current highthroughput methods are optimized for proliferating, easily transferable cell lines and not for postmitotic neurons [42, 139]. To reduce the technical difficulties, Niedringhaus et al. worked out a transferable raft miniculturing practice for neuron cultures which provide sufficient experimental material for microvolume reactions and appropriate sample sizes [42]. Notably, the cell viability, sample-to-sample reproducibility, and screening potential proved to be higher than those on conventional well-plates.

An additional hampering factor might be the timeconsuming derivation and characterization of neurons from somatic cells accompanied by several technical pitfalls. Research industry offers several genetically modified iPSCderived neurons which can be a faster model for drug development after thorough validation [125].

6.6. Double Standard: Stable and Flexible. The iPSC/iNC line generation requires stem cell-like, incompact chromatin and multiple epigenetic rearrangements. Controversially, scientists agree that a high-quality iPSC/iNC assay conserves the donor's genetic information (including parental imprinting). The NIMH meeting participants, and many since then, conceived a reasonable concern on the genetic (un)stability of induced cell lines [10].

Previously, chromosome aberrations, gene deletions/ duplications, and point mutations were thought to be the 
main mechanisms responsible for de novo mutations during the pluripotent state. However, multiple studies demonstrated that iPSCs and iNPCs exhibit chromosomal stability (one examined 1700 stem cell cultures [140]). Per contra, copy number variations (CNVs) show significant incidence $[23,112]$. All of the investigated cell lines gained CNVs and the CNV signature of the iPSCs differed from both somatic parent cells and iNCs. This indicates that de novo mutations may appear through the somatic cell-iPSC-NPC-neuron transitions. On the other hand, mosaicism for CNVs is also common in vivo: it represents $0.12-7.3 \%$ of intraindividual genomic variability [141].

Keeping in mind that CNVs are known to influence clonal selection in cell cultures [142] and participate in the etiology of several human diseases, including neurodevelopmental disorders (SCZ [143], ASD [31], and ADHD [144]), the iPSC/iNC quality control calls for reconsideration. It was always supposed but, recently, Kang et al. proved that genetic integrity is highly influenced by the reprogramming technique, and DNA nonintegrating protocols are safer [145]. The previously recommended karyotyping is not sufficient for ensuring validity; instead, DNA sequencing of the source and generated cells is desirable.

6.7. The Neuronal Genome: Imperfectly Imitable? Somatic mosaicism affects the brain also: brain-only or brain regiononly somatic mutations, chromosome aneuploidy, microdeletions, or retrotransposon dynamics have been detected in post mortem brain tissue. Presumably, these neural genomic variations contribute to the functional heterogeneity of brain cells and also to the development of neuropsychiatric disorders [146]. For instance, L1 retroelements (the only human retrotransposon with autonomous activity) exhibit increased copy number in adult NPCs compared to nonbrain cells and are known to be associated with Rett syndrome [147]. Bundo et al. examined the L1 signature in SCZ using multiple model systems [18]. First, they showed that L1 copy number is increased in the prefrontal cortical neurons of SCZ patients compared to controls and autologous nonbrain cells. To answer the question whether this ensues from hereditary or environmental factors, the group assessed the L1 profile in an environment-induced SCZ animal model and in iNCs derived from SCZ patients with rare mutation of large effect (22q11 deletion). The genomic DNA of the mouse brain exhibited the consequences of high L1 activity and they also detected increased L1 insertion rate during in vitro neurogenesis. Moreover, brainspecific L1 insertion sites were near or in genes involved in synaptic functions and neuropsychiatric disorders supporting the possible pathognomic role of retrotransposition events. For conclusion, we can speculate that increased L1 dynamics by environmental and/or genetic factors may increase the susceptibility to neurodevelopmental disorders by disrupting synaptic and schizophrenia related genes in neurons.

This outstanding experimental design reassures the validity of iPSCs/iNCs in modeling neurodevelopment and calls for further studies on the neuronal genome.
6.8. Designation of Origin. Besides the above-mentioned somatic mosaicism, cellular commitment and in vivo cellaging raise the following question: Does the source cell type matter? Neuropsychiatric studies apply the most frequently easy-to-obtain lymphocytes, fibroblasts, and keratinocytes; but, theoretically, all somatic cells can be reprogrammed or transdifferentiated into iPSCs and iNCs.

These somatic cells widely vary in their epigenetics, proliferative potential, and the rate of cellular aging. Previous studies found that the intrinsic properties of the source cells, that is, stage of differentiation [148], senescence [149], tissue type [150], and number of passages [151], influence the efficacy of reprogramming. Furthermore, the generated cells display a residual gene expression pattern of the source cell type referring to "incomplete reprogramming" and result in variability among iPSCs from different tissue samples [152].

Chen et al. speculated that induced cell lines may retain and transmit transcriptional/epigenetic marks from the source tissue to the differentiated cells; therefore, they carried out whole transcriptome analysis in neurons gained from fibroblasts or dental pulp [20]. Notably, they found 63 differentially expressed genes, including a glutamate receptor, choline, GABA, and glycine transporter. Striking differences were found in the expression of the SLITRK2 gene (associated with BPD and ASD), multiple HOX genes, and a set of transcription factors. Pathway analysis revealed that neurological disease/schizophrenia related gene sets are the most affected [20]. Unfortunately, the interpretation of the results might be problematic since the dental pulp was obtained from a 12 -year-old male subject, while fibroblasts originated from a 30-year-old female and a 58-year-old male. However, the experiment is highly noteworthy: even if we conclude that the source cell type or the donor's age passed down through generations and affected the gene expression of the iNCs, it urges for further research.

6.9. Patients Ill Sorted? One of the main research principles is representativeness: the sample has to be an unbiased illustration of the studied population. Psychiatric diagnostic categories cover a wide range of patients with great heterogeneity in etiological factors, symptomatology, disease progression, and therapy response. This diversity issued several difficulties in experimental and clinical settings during the past decades.

Now, the overlook is changing: DSM- 5 omitted the previous SCZ subtypes defined by clinical symptoms [153]. Two years later, the Consortium on the Genetics of Schizophrenia (COGS) postulated that endophenotypes could provide a more negotiable approach of patient categorization for clinical and research purposes [154]. Endophenotypes, for example, cognitive dysfunction, EEG-markers, or brain imaging phenotypes, are quantitative laboratory based measures with a same level of heritability as SCZ itself [155]. They can be linked to certain genotypes, cellular phenotypes, psychopathologies, and functional impairments and fill the gene to phene gap. The NIMH workshop in 2012 also addressed the critical step of patient selection and recommended subject recruitment based on comprehensive clinical, genetic, and cellular characterization. 
6.10. The Art of Design. Disrupted in schizophrenia 1 (DISC1) gene is known to be involved in fetal and adult neurodevelopment and neuroplasticity, and its variations are highly associated with a wide range of mental disorders: SCZ, BPD, MDD, and ASD as well [156]. Presumably, it predisposes to endophenotypes which can manifest themselves in different clinical syndromes depending on the genetic and environmental cofactors [157].

Wen et al. aimed to study cellular consequences of the DISC1 mutation [54]. They derived iPSC lines from a carrier pedigree: one SCZ and one MDD patient with a DISC1 frameshift mutation, two unaffected family members without the mutation, and one additional control: an unrelated healthy subject. The DISC1 mutation caused functional synaptic transmission deficits in the glutamatergic neurons via pervading transcriptome alterations. To challenge their hypothesis on the primary pathognomic effect of the DISC1 mutation, the research group repeated the measures with the isogenic pairs of the cell lines, generated by DNA editing. Over and above the obvious values of their results in understanding DISC1 related pathophysiology and filling the gap between the genotype and the clinical picture with a cellular endophenotype, this work provides a great example for careful, overthought study design.

Considering that patient iPSC/iNC studies are usually conducted on small sample sizes (1-5 persons/group) and advancements are trending towards personalized medicine, careful case-control matching in iPSC/iNC research is crucial to maintain validity and reliability. When monogenic diseases (e.g., Rett and Fragile $\mathrm{X}$ syndrome) are investigated, the gender, age, and/or genotype based selection of healthy individuals is sufficient. Per contra, in case of polygenic neuropsychiatric disorders with intermediate heritability (30$70 \%$ ) other designs are noteworthy since the combined effect of all disease-related and irrelevant alleles manifests itself in the dish [158].

For instance, pedigree-studies allow identifying heritability factors. Inclusion of affected and unaffected family members reduces the genetic noise (heterogeneity) and thus the risk of type I error. While a second, independent control group from out-of-pedigree healthy subjects helps to oversee the potential effects from family genetic background. Isogenic pairs produced by genetic correcting technologies (e.g., TALE nuclease [159], zinc finger nuclease [160], homologous recombination [161], or CRISPR [162]) allow a cell line to be its own control and enables the targeted testing of single gene effects via excluding (epi)genetic diversity. In some cases (e.g., CNVs, trinucleotide repeats, and X-linked disorders), in vivo or in vitro mosaicism provides isogenic controls [26].

6.11. Psyche in the Dish? The question is not sceptic by all means. How can we validate a specific iPSC/iNC line for modeling a psychiatric disorder? Chromosomal and cellular marker characterization and evaluation of genetic stability are only the base of quality control. Having differentiated neurons does not evidence a model for neurophysiology or disease neuropathology.

Two research groups aimed the validation of a commercially available neural cell line for further studies on ASD and neurodegenerative diseases $[1,24,125]$. The corroboration included whole transcriptome analysis, receptor and ion channel profiling, and detailed electrophysiological characterization. According to their results, the cells display early developmental neural phenotype and express the majority of ASD associated genes and thus this cell line might be utilized as control in comparison to patient-derived cells or suitable for isogenic mutant-wild type pair generation.

Interestingly, many of the ASD-related genes showed changes along the culturing process [24], and Belinsky et al. also detected specific temporal manner in the genes of interest (DISC1, DTNBP1, GAD1, PAX6, FOXP1, FOXP2, vGLUT1, and COMT) [13]. This sounds reasonable since the mentioned genes are linked to neurodevelopment, synaptic transmission, and intracellular signaling, functions highly implicated during neural maturing. However, these observations are worthy of note, suggesting that timing can easily enhance or undermine the validity of the experiment, especially when the most frequently used early neural and forebrain markers (PAX6 and vGLUT1 and GAD1) or canonical disease genes (DISC1 and COMT) are the subjects. Concordantly, Wen et al. found that DISC1 mutation caused structural anomalies and synaptic dysfunction attenuate around postdifferentiation weeks 4-6 [54].

Usually, experimental data can be embedded into previous results and legitimized by the cited literature. Considering the novelty of the field and the limited number of research papers, iPSC/iNC researchers can rarely expect their model's validation from preceding experiments. Comparing the results with animal model and human postmortem findings can offer pivots; however, dissimilarities might ensue from differences between species and cell types since we are matching pure neuron cultures with tissues [41].

6.12. Grafting Neurons for Psychiatric Treatment? Three future applications of iPSCs and iNCs are most commonly predicted [24]. As model systems, they can help us understand the cellular pathophysiology of neuropsychiatric disorders and reveal genotype-phenotype correlations. Highthroughput cellular screening assays may provide novel targets in drug development; and, finally, iPSC-derived cells may play a role in regenerative medicine.

Induced or embryonic PSC-based replacement therapy research is one of the most expeditiously developing fields of medicine. Currently, clinical trials are in progress in macula degeneration, type I diabetes mellitus, ischemic heart failure, and spinal cord injury [163]. Preclinical results are also promising in Parkinson's and Alzheimer's disease, neurodegenerative disorders with well-defined pathological and functional alterations and cellular loss (recently reviewed in $[163,164])$. And what about other psychiatric disorders? Animal models can provide us with hints about this application.

For instance, SCZ has multifactorial origin which pervades the whole connectivity during neurodevelopment and results in poorly understood brain pathology. Human and animal research suggest that cortical and hippocampal inhibitory interneuron deficit contributes to the dopaminergic system dysfunction, thus the positive symptoms of 
SCZ [165]. In rodents, embryonic and induced human and nonhuman NPC grafts survive, proliferate, migrate, and differentiate spontaneously into pyramidal or GABAergic neurons [166-168]. The integration of these inhibitory neurons into the host neural circuits successfully modulated the hyperactive dopaminergic system and the behavior analogous of positive symptoms in a mouse SCZ model. While these engraftment experiments are perplexing and can contribute substantially to our understanding of the neurobiology of psychiatric disorders, they do not necessarily forecast engraftment of in vitro differentiated neurons as a feasible approach for the treatment of psychiatric disorders in the near future. Disease pathology remains poorly understood and the clinical hurdles are also numerous.

6.13. Open Access in Stem Cell Based Disease Modeling. The NIMH workshop addressed one more intensively discussed, still actual issue: information and resource sharing which is especially meaningful in the rapidly developing scientific fields such as iPSC/iNC research [10]. The participants argued that open sharing of data and standardization of iPSC/iNC generation and validation protocols are essential for improving experimental reproducibility.

It is well known that open sharing and collaborative environment empower knowledge circulation and thus fuels innovation and discovery. Therefore, decision-makers took significant steps to implement the open access policy: in 2015, both the European Commission and the US Congress supported the proposal that articles on publicly funded researches have to be freely available for anyone $[169,170]$. We were curious if this open access movement is observable in the iPSC/iNC literature. We found that $73 \%$ of the reviewed research papers are freely accessible ( 35 from 48 articles, listed in Table 2(a)).

The other basis of open science is resource sharing. Growing number of cell banks collect somatic cells and/or iPSCs from patients with neuropsychiatric disorders and assure accessibility for the scientific community on request $[17,171]$. Such (inter)national consortiums and collaborations provide standardized databases and methodology, increased, homogenous sample quality, and possibility to study rare genetic or phenotypic variations, affected families, or-as seen in Sweden-isogenic cell line pairs from monozygotic twins [172].

\section{Tools in Our Hands: We Have Nothing to Fear}

The ideal cell culture model for in vitro experiments meets the following requirements: (1) it is easily obtainable via minimal invasive intervention; (2) the initiation and maintenance of the cell culture are not extremely labor intensive or timeconsuming; (3) the fact that differentiation, if necessary, can be directed and monitored; (4) the cell line reserves proliferative, self-sustaining capacity over passages; (5) and this is done with genetic stability; (6) it exhibits similar (or identical) pathophysiological features to the diseased tissue in vivo; and (7), last but not least, the method provides enough experimental material at reasonable expenses.
Revising the currently available in vitro systems, the words of Salvador Dali flash on: "Have no fear of perfection - you'll never reach it." Ideal in vitro model cannot exist, but depending on the concept of the research and the accessible resources one can choose the most optimal from the following.

Extended literature discusses peripheral cells, such as blood leukocytes and dermal fibroblasts, as potential in vitro models and biomarker sources of mental disorders. They are relatively easy-to-obtain, robust cell lines and share receptor and signaling pathway similarities with CNS cells [173-175]. Fibroblasts have self-maintaining capacity $a b$ ovo and maintain homogeneity between passages 5 and 20 [176]. Freshly isolated leukocytes are appropriate even for bedside functional assays or can be immortalized for culturing but represent poor genetic stability [177]. Finally, we cannot disregard that peripheral cells do not permit the examination of specific neural phenomena (e.g., electrophysiology, microarchitecture, and neurodevelopment).

Human primary neural cultures, initiated from brain biopsies, are barely applied to neuroscience. They require special conditions and lack self-maintaining capacity; therefore, the amount of experimental material is restricted. Furtherly, the cells are already tainted with life-long in vivo effects, which balks the expression of the naïve endophenotype.

Since 2006, when Takahashi and Yamanaka showed that the expression of four transcription factors can reprogram adult somatic cells into an earlier ontogenic state [98], induced pluripotent stem cells (iPSCs) and iPSC-derived cell lines became one of the most studied and advancing fields of medicine; but, as seen above, we still face numerous technical or theoretical issues.

\section{Can We Make a Long Story Short?}

The main concerns about iPSC-research, that is, time and resource demands, genetic instability, epigenetic changes, and populational heterogeneity, might be partially overcome by omission of the pluripotent state and direct transdifferentiation of somatic cells into completely different cell types. After Takahashi and Yamanaka introduced the method of cellular reprogramming, researchers started to work on new protocols to establish neural cultures from somatic cells by direct cell lineage conversion. Vierbuchen et al. were the first who successfully transdifferentiated mouse fibroblasts into functional neurons [95]. Since then, they and others showed that human somatic cells (e.g., fibroblasts [134], blood cells [178], and hepatocytes [179]) can be transdifferentiated into neural progenitor cells [180] or postmitotic neurons via forced expression or exogenous addition of transcription factors, microRNAs, or small molecules. Moreover, one single factor is enough to induce neural cell fate [181] and cell type-specific factors allow the generation of dopaminergic [182], motor neurons [183] or oligodendrocytes [184]. The induced neural cells resemble in vivo neurons in their functional, electrophysiological, translational characteristics and form functional synapses; thus, they can be valuable in vitro models of neuropsychiatric disorders. However, as of yet only one experiment has been published on direct 
reprogramming in the context of psychiatric disorders. Wang et al. [53] used lentiviral transduction with a construct expressing miR-9/9* -124, NEUROD2, ASCL1, and MYT1L to transdifferentiate fibroblasts from bipolar patients responsive or unresponsive to lithium medication, the gold standard of mood-stabilizing treatments. iNCs derived from lithiumresponders and lithium-nonresponders demonstrated different cell adhesion characteristics. This innovative approach demonstrates the translation potential and "nearly bedside" application of transdifferentiation-based assays.

The greatest advantage of direct lineage conversion is bypassing pluripotent states and avoiding potential oncogenicity as a consequence. On the other hand, this stability, that is, lack of self-renewal and potential amplification, might be a drawback in laboratory research and regenerative medicine. Besides, the possibility of residuals from in vivo cellular senescence and epigenetic memories inherited from the parental tissue deserves further considerations [185].

\section{The Undeservedly Neglected: Glia-Associated Pathologies}

Vast majority of psychiatric research deals with neurons but they account for only about one-third of human brain cells. Glial cells are responsible for maintaining brain homeostasis and neural well-being. They provide lactate as source of energy, regulate the redox balance, metabolic clearance, and CNS immunology $[186,187]$, and play crucial role in directing neuronal migration, neurite outgrowth, and synaptic pruning [188]. Additionally, astrocytes are in bidirectional cross talk with neurons, and glial neurotransmission has been proposed to be involved in several mental functions (e.g., memory, motor control, and decision making) and neuropsychiatric disorders [189]. Postmortem human and animal studies proved that glial cells contribute to the development and progression of psychiatric disorders and can be potential therapeutic targets [190, 191].

NPCs derived from iPSCs can be differentiated into glial precursors and mature astrocytes and oligodendrocytes [192, 193]. Microglial-like cells are even more easily obtainable due to their mesodermal origin, and circulating monocytes can be transdifferentiated within 14 days [194]. These cell lines are underrepresented in psychiatric research, only used as a feeder/supporting layer for the neurons. Per contra, they are intensively studied as potential disease models and therapeutical targets in neurological disorders, for example, Huntington disease [195], amyotrophic lateral sclerosis [196] or congenital hypomyelination [197], and intellectual disability [198].

Yet, we can find a great example: how neuron-glia cocultures can help to answer whether certain glia-associated pathologies are pathognomic factors, additive part of the endophenotype, or reactive (beneficial) response to the neural dysfunction. Williams et al. differentiated astrocytes and neurons from iPSCs of Rett syndrome patients and demonstrated that mutant astrocytes have non-cell-autonomous adverse effects both on cocultured mutant and on healthy neurons [55]. The group also proved that this impact is directed by the extracellular environment since the astrogliaconditioned media took the same detrimental effect on the morphology and function of wild type and mutant neuronal cultures. The adverse effects could be rescued by IGF-1 supplementation which underpins the ongoing IGF-1-based clinical trials in the treatment of Rett syndrome. Surprisingly, the efficiency of IGF-1 supplementation depended on the genotype of the astrocyte, which calls for further pharmacogenetic studies. According to these findings, the effects of the mutations in the astrocytes and neurons appear to be additive. Similar innovative studies will unravel the complex association of human neurons and glial cells in healthy and diseased brains.

\section{Limitations}

On the whole, results are reassuring. Those who reported differentiation and maturation anomalies in iNCs from patients with neurodevelopmental disorders [37, 41, 43, 45, 46, 49] are more than those who could not detect alterations during differentiation [14] or in electrophysiological properties [13, 54]. However, there are some noteworthy study design and publication biases.

(1) Scalability represents a central issue for stem cell based disease modeling. Ideally, the number of cell lines derived from specific patients could be scaled up to numbers typical for clinical studies, meaning tens or hundreds of patients. Realistically seen, today this is not feasible. However we must keep in mind that this method is in its infancy and the task remains the exploration of cell lines derived from genetically and clinically well characterized individuals.

(2) A priori hypotheses influence the objectives and the measured parameters. Differentiation is intensively monitored in these diseases; therefore, such small-scale variations like $12 \%$ nuclear size differences can be detected [11], but less is known about the transcriptome or receptor profile alterations. (3) Majority of the studies examined the iPSC/iNC lines at rest in monotonous environments which might be detrimental during neurodevelopment, that is, specialization for signal detection and transmission. Therefore environmental challenges could enhance and reveal additional differences as showed in $[29,36]$. (4) Great proportion of these differences might affect synaptic transmission which is understudied in iPSC/iNC models; however, novel visualization and high-content screening technologies might bring advances to this field. For instance, functional assays of DISC1 mutant cells, namely, spontaneous synaptic current measurement and synaptic vesicle staining in living cells, revealed synaptic vesicle release defect which were also observable in the transcriptome [54].

(5) Similarly, there is a rate shift towards pervasive, monogenic neurodevelopmental syndromes with early-childhood presentation and well-defined clinical and genetical picture. The nature of these syndromes differs substantially from the most frequent adult psychiatric disorders which are multifactorial with less robust pathology and might show themselves in the connectome and not on the single-cell level. (6) Additionally, in case of polygenic disorders, the discrete phenotypic alterations might be very small, presented on a 
continuous spectrum. In contrast, iPSC/iNC studies usually recruit psychiatric patients with rare mutations of large effects. Selection of severe cases based on the polygenic score method could be a possible approach to overcome this limitation.

\section{Take Home Messages}

This review demonstrates the unprecedented possibilities offered by iPSC based in vitro disease modeling in psychiatry, a field of medicine awaiting major developments. It is striking how fragmented our understanding is about molecular disease pathways underlying SCZ, BPD, and ASD and how high the level of unmet needs is among patients suffering from these disorders. Incomplete therapeutic response, therapyresistance, and cognitive deterioration are all major hurdles in the treatment of psychiatric patients. In vitro disease modeling will help us with diagnostics by demonstrating the heterogeneity within clinical disease groups in terms of molecular disease mechanisms. This question, whether similar clinical phenotypes share molecular foundations or are rather determined by a final common pathway, remains a central idea in psychiatry. Stem cells could also contribute to treatment by paving the way to personalized pharmacological treatment and drug screening as detailed in the review. The prospects of stem cell based disease modeling cannot be exactly foreseen; however, based on the past few years' developments we can envisage major breakthroughs in stem cell based psychiatry for the benefit of our patients.

\section{Conflict of Interests}

The authors declare that there is no conflict of interests regarding the publication of this paper.

\section{Acknowledgments}

The study was supported by the Hungarian National Research Fund and the Hungarian Brain Research Program (Grants OTKA-PD 83876 and NAP-B KTIA_NAP_13-2014-0011 to János M. Réthelyi).

\section{References}

[1] C. Arango, D. Fraguas, and M. Parellada, "Differential neurodevelopmental trajectories in patients with early-onset bipolar and schizophrenia disorders," Schizophrenia Bulletin, vol. 40, supplement 2, pp. S138-S146, 2014.

[2] J. Olesen, A. Gustavsson, M. Svensson, H. U. Wittchen, and B. Jönsson, "The economic cost of brain disorders in Europe," European Journal of Neurology, vol. 19, no. 1, pp. 155-162, 2012.

[3] US FDA, Report on Challenge and Opportunity on the Critical Path to New Medical Products, US FDA, 2004.

[4] P. F. Sullivan, K. S. Kendler, and M. C. Neale, "Schizophrenia as a complex trait: evidence from a meta-analysis of twin studies," Archives of General Psychiatry, vol. 60, no. 12, pp. 1187-1192, 2003.

[5] R. A. Shih, P. L. Belmonte, and P. P. Zandi, "A review of the evidence from family, twin and adoption studies for a genetic contribution to adult psychiatric disorders," International Review of Psychiatry, vol. 16, no. 4, pp. 260-283, 2004.

[6] W. Deng, X. Zou, H. Deng et al., "The relationship among genetic heritability, environmental effects, and autism spectrum disorders: 37 pairs of ascertained twin study," Journal of Child Neurology, 2015.

[7] S. A. McCarroll, G. Feng, and S. E. Hyman, "Genome-scale neurogenetics: methodology and meaning," Nature Neuroscience, vol. 17, no. 6, pp. 756-763, 2014.

[8] S. Dong, M. F. Walker, N. J. Carriero et al., "De novo insertions and deletions of predominantly paternal origin are associated with autism spectrum disorder," Cell Reports, vol. 9, no. 1, pp. 16-23, 2014.

[9] T. M. Kranz, S. Harroch, O. Manor et al., "De novo mutations from sporadic schizophrenia cases highlight important signaling genes in an independent sample," Schizophrenia Research, vol. 166, no. 1-3, pp. 119-124, 2015.

[10] D. M. Panchision, "Meeting report: using stem cells for biological and therapeutics discovery in mental illness, April 2012," Stem Cells Translational Medicine, vol. 2, no. 3, pp. 217-222, 2013.

[11] G. Ananiev, E. C. Williams, H. Li, and Q. Chang, "Isogenic pairs of wild type and mutant induced pluripotent stem cell (iPSC) lines from rett syndrome patients as in vitro disease model," PLoS ONE, vol. 6, no. 9, Article ID e25255, 2011.

[12] S. Bavamian, N. Mellios, J. Lalonde et al., "Dysregulation of miR-34a links neuronal development to genetic risk factors for bipolar disorder," Molecular Psychiatry, vol. 20, no. 5, pp. 573584, 2015.

[13] G. S. Belinsky, M. T. Rich, C. L. Sirois et al., "Patch-clamp recordings and calcium imaging followed by single-cell PCR reveal the developmental profile of 13 genes in iPSC-derived human neurons," Stem Cell Research, vol. 12, no. 1, pp. 101-118, 2014.

[14] C. Boissart, A. Poulet, P. Georges et al., "Differentiation from human pluripotent stem cells of cortical neurons of the superficial layers amenable to psychiatric disease modeling and highthroughput drug screening," Translational Psychiatry, vol. 3, article e294, 2013.

[15] K. J. Brennand, A. Simone, J. Jou et al., "Modelling schizophrenia using human induced pluripotent stem cells," Nature, vol. 473, no. 7346, pp. 221-225, 2011.

[16] K. Brennand, J. N. Savas, Y. Kim et al., "Phenotypic differences in hiPSC NPCs derived from patients with schizophrenia," Molecular Psychiatry, vol. 20, pp. 361-368, 2015.

[17] D. J. Brick, H. E. Nethercott, S. Montesano et al., "The autism spectrum disorders stem cell resource at children's hospital of orange county: implications for disease modeling and drug discovery," Stem Cells Translational Medicine, vol. 3, no. 11, pp. 1275-1286, 2014.

[18] M. Bundo, M. Toyoshima, Y. Okada et al., "Increased L1 retrotransposition in the neuronal genome in schizophrenia," Neuron, vol. 81, no. 2, pp. 306-313, 2014.

[19] H. M. Chen, C. J. DeLong, M. Bame et al., "Transcripts involved in calcium signaling and telencephalic neuronal fate are altered in induced pluripotent stem cells from bipolar disorder patients," Translational Psychiatry, vol. 4, article e375, 2014.

[20] J. Chen, M. Lin, J. J. Foxe et al., "Transcriptome comparison of human neurons generated using induced pluripotent stem cells derived from dental pulp and skin fibroblasts," PLOS ONE, vol. 8, no. 10, Article ID e75682, 2013. 
[21] A. Y. L. Cheung, L. M. Horvath, D. Grafodatskaya et al., "Isolation of MECP2-null Rett Syndrome patient hiPS cells and isogenic controls through X-chromosome inactivation," Human Molecular Genetics, vol. 20, no. 11, Article ID ddr093, pp. 2103-2115, 2011.

[22] C.-H. Chiang, Y. Su, Z. Wen et al., "Integration-free induced pluripotent stem cells derived from schizophrenia patients with a DISC1 mutation," Molecular Psychiatry, vol. 16, no. 4, pp. 358360, 2011.

[23] N. L. L. Corrales, K. Mrasek, M. Voigt, T. Liehr, and N. Kosyakova, "Copy number variations (CNVs) in human pluripotent cell-derived neuroprogenitors," Gene, vol. 506, no. 2, pp. 377-379, 2012.

[24] J. L. Dage, E. M. Colvin, A. Fouillet et al., "Pharmacological characterisation of ligand- and voltage-gated ion channels expressed in human iPSC-derived forebrain neurons," Psychopharmacology, vol. 231, no. 6, pp. 1105-1124, 2014.

[25] B. A. DeRosa, J. M. Van Baaren, G. K. Dubey et al., "Derivation of autism spectrum disorder-specific induced pluripotent stem cells from peripheral blood mononuclear cells," Neuroscience Letters, vol. 516, no. 1, pp. 9-14, 2012.

[26] M. E. Doers, M. T. Musser, R. Nichol et al., "IPSC-derived forebrain neurons from FXS individuals show defects in initial neurite outgrowth," Stem Cells and Development, vol. 23, no. 15, pp. 1777-1787, 2014.

[27] N. D. Germain, P.-F. Chen, A. M. Plocik et al., "Gene expression analysis of human induced pluripotent stem cell-derived neurons carrying copy number variants of chromosome 15q11q13.1," Molecular Autism, vol. 5, article 44, 2014.

[28] K. Griesi-Oliveira, A. Acab, A. R. Gupta et al., "Modeling nonsyndromic autism and the impact of TRPC6 disruption in human neurons," Molecular Psychiatry, 2014.

[29] K. Hashimoto-Torii, M. Torii, M. Fujimoto et al., "Roles of heat shock factor 1 in neuronal response to fetal environmental risks and its relevance to brain disorders," Neuron, vol. 82, no. 3, pp. 560-572, 2014.

[30] V. Hook, K. J. Brennand, Y. Kim et al., "Human iPSC neurons display activity-dependent neurotransmitter secretion: aberrant catecholamine levels in schizophrenia neurons," Stem Cell Reports, vol. 3, no. 4, pp. 531-538, 2014.

[31] B. H.-Y. Chung, V. Q. Tao, and W. W.-Y. Tso, "Copy number variation and autism: new insights and clinical implications," Journal of the Formosan Medical Association, vol. 113, no. 7, pp. 400-408, 2014.

[32] J. F. Krey, S. P. Paşca, A. Shcheglovitov et al., "Timothy syndrome is associated with activity-dependent dendritic retraction in rodent and human neurons," Nature Neuroscience, vol. 16, no. 2, pp. 201-209, 2013.

[33] D. Kumari, M. Swaroop, N. Southall, W. Huang, W. Zheng, and K. Usdin, "High-throughput screening to identify compounds that increase fragile $\mathrm{X}$ mental retardation protein expression in neural stem cells differentiated from fragile $\mathrm{X}$ syndrome patient-derived induced pluripotent stem cells," Stem Cells Translational Medicine, vol. 4, no. 7, pp. 800-808, 2015.

[34] J. Larimore, P. V. Ryder, K.-Y. Kim et al., "MeCP2 regulates the synaptic expression of a Dysbindin-BLOC-1 network component in mouse brain and human induced pluripotent stem cellderived neurons," PLoS ONE, vol. 8, no. 6, Article ID e65069, 2013.

[35] M. Lin, A. Hrabovsky, E. Pedrosa, T. Wang, D. Zheng, and H. M. Lachman, "Allele-biased expression in differentiating human neurons: implications for neuropsychiatric disorders," PLoS ONE, vol. 7, no. 8, Article ID e44017, 2012.

[36] M. Lin, D. Zhao, A. Hrabovsky, E. Pedrosa, D. Zheng, and H. M. Lachman, "Heat shock alters the expression of schizophrenia and autism candidate genes in an induced pluripotent stem cell model of the human telencephalon," PLoS ONE, vol. 9, no. 4, Article ID e94968, 2014.

[37] J. Liu, K. A. Kościelska, Z. Cao et al., "Signaling defects in iPSCderived fragile X premutation neurons," Human Molecular Genetics, vol. 21, no. 17, Article ID dds207, pp. 3795-3805, 2012.

[38] J. M. Madison, F. Zhou, A. Nigam et al., "Characterization of bipolar disorder patient-specific induced pluripotent stem cells from a family reveals neurodevelopmental and mRNA expression abnormalities," Molecular Psychiatry, vol. 20, no. 6, pp. 703-717, 2015.

[39] M. Maekawa, K. Yamada, M. Toyoshima et al., "Utility of scalp hair follicles as a novel source of biomarker genes for psychiatric illnesses," Biological Psychiatry, vol. 78, no. 2, pp. 116-125, 2015.

[40] M. C. N. Marchetto, C. Carromeu, A. Acab et al., "A model for neural development and treatment of rett syndrome using human induced pluripotent stem cells," Cell, vol. 143, no. 4, pp. 527-539, 2010.

[41] M. Maschietto, A. C. Tahira, R. Puga et al., "Co-expression network of neural-differentiation genes shows specific pattern in schizophrenia," BMC Medical Genomics, vol. 8, article 23, 2015.

[42] M. Niedringhaus, R. Dumitru, A. M. Mabb et al., “Transferable neuronal mini-cultures to accelerate screening in primary and induced pluripotent stem cell-derived neurons," Scientific Reports, vol. 5, article 8353, 2015.

[43] S. P. Paşca, T. Portmann, I. Voineagu et al., "Using iPSCderived neurons to uncover cellular phenotypes associated with Timothy syndrome," Nature Medicine, vol. 17, no. 12, pp. 16571662, 2011.

[44] B. D. S. Paulsen, S. C. Cardoso, M. P. Stelling, D. V. Cadilhe, and S. K. Rehen, "Valproate reverts zinc and potassium imbalance in schizophrenia-derived reprogrammed cells," Schizophrenia Research, vol. 154, no. 1-3, pp. 30-35, 2014.

[45] E. Pedrosa, V. Sandler, A. Shah et al., "Development of patientspecific neurons in schizophrenia using induced pluripotent stem cells," Journal of Neurogenetics, vol. 25, no. 3, pp. 88-103, 2011.

[46] O. Robicsek, R. Karry, I. Petit et al., "Abnormal neuronal differentiation and mitochondrial dysfunction in hair follicle-derived induced pluripotent stem cells of schizophrenia patients," Molecular Psychiatry, vol. 18, no. 10, pp. 1067-1076, 2013.

[47] P. Roussos, A. C. Mitchell, G. Voloudakis et al., "A role for noncoding variation in schizophrenia," Cell Reports, vol. 9, no. 4, pp. 1417-1429, 2014.

[48] A. Shcheglovitov, O. Shcheglovitova, M. Yazawa et al., "SHANK3 and IGF1 restore synaptic deficits in neurons from 22q13 deletion syndrome patients," Nature, vol. 503, no. 7475, pp. 267-271, 2013.

[49] S. D. Sheridan, K. M. Theriault, S. A. Reis et al., "Epigenetic characterization of the FMR1 gene and aberrant neurodevelopment in human induced pluripotent stem cell models of fragile X syndrome," PLoS ONE, vol. 6, no. 10, Article ID e26203, 2011.

[50] S. Shi, C. Leites, D. He et al., "MicroRNA-9 and microRNA326 regulate human dopamine $\mathrm{D} 2$ receptor expression, and the microrna-mediated expression regulation is altered by a genetic variant," The Journal of Biological Chemistry, vol. 289, no. 19, pp. 13434-13444, 2014. 
[51] Y. Tian, I. Voineagu, S. P. Paşca et al., "Alteration in basal and depolarization induced transcriptional network in iPSC derived neurons from Timothy syndrome," Genome Medicine, vol. 6, no. 10, article 75, 2014.

[52] A. Topol, S. Zhu, N. Tran, A. Simone, G. Fang, and K. J. Brennand, "Altered WNT signaling in human induced pluripotent stem cell neural progenitor cells derived from four schizophrenia patients," Biological Psychiatry, vol. 78, no. 6, pp. e29-e34, 2015.

[53] J. L. Wang, S. M. Shamah, A. X. Sun, I. D. Waldman, S. J. Haggarty, and R. H. Perlis, "Label-free, live optical imaging of reprogrammed bipolar disorder patient-derived cells reveals a functional correlate of lithium responsiveness," Translational Psychiatry, vol. 4, article e428, 2014.

[54] Z. Wen, H. N. Nguyen, Z. Guo et al., "Synaptic dysregulation in a human iPS cell model of mental disorders," Nature, vol. 515, no. 7527, pp. 414-418, 2014.

[55] E. C. Williams, X. Zhong, A. Mohamed et al., "Mutant astrocytes differentiated from Rett syndrome patients-specific iPSCs have adverse effects on wild-type neurons," Human Molecular Genetics, vol. 23, no. 11, Article ID ddu008, pp. 2968-2980, 2014.

[56] K.-J. Yoon, H. N. Nguyen, G. Ursini et al., "Modeling a genetic risk for schizophrenia in iPSCs and mice reveals neural stem cell deficits associated with adherens junctions and polarity," Cell Stem Cell, vol. 15, no. 1, pp. 79-91, 2014.

[57] D. X. Yu, F. P. Di Giorgio, J. Yao et al., "Modeling hippocampal neurogenesis using human pluripotent stem cells," Stem Cell Reports, vol. 2, no. 3, pp. 295-310, 2014.

[58] L. Zeng, P. Zhang, L. Shi, V. Yamamoto, W. Lu, and K. Wang, "Functional impacts of NRXN1 knockdown on neurodevelopment in stem cell models," PLoS ONE, vol. 8, no. 3, Article ID e59685, 2013.

[59] A. Acab and A. R. Muotri, "The use of induced pluripotent stem cell technology to advance autism research and treatment," Neurotherapeutics, vol. 12, no. 3, pp. 534-545, 2015.

[60] S. Aigner, T. Heckel, J. D. Zhang, L. C. Andreae, and R. Jagasia, "Human pluripotent stem cell models of autism spectrum disorder: emerging frontiers, opportunities, and challenges towards neuronal networks in a dish," Psychopharmacology, vol. 231, no. 6, pp. 1089-1104, 2014.

[61] K. Ardhanareeswaran, G. Coppola, and F. Vaccarino, "The use of stem cells to study autism spectrum disorder," Yale Journal of Biology and Medicine, vol. 88, no. 1, pp. 5-16, 2015.

[62] K. J. Brennand and F. H. Gage, "Modeling psychiatric disorders through reprogramming," Disease Models and Mechanisms, vol. 5, no. 1, pp. 26-32, 2012.

[63] K. J. Brennand, M. A. Landek-Salgado, and A. Sawa, "Modeling heterogeneous patients with a clinical diagnosis of schizophrenia with induced pluripotent stem cells," Biological Psychiatry, vol. 75, no. 12, pp. 936-944, 2014.

[64] J. D. Buxbaum and P. Sklar, "Human induced pluripotent stem cells: a new model for schizophrenia?" Cell Stem Cell, vol. 8, no. 5, pp. 461-462, 2011.

[65] T. Chailangkarn, A. Acab, and A. R. Muotri, "Modeling neurodevelopmental disorders using human neurons," Current Opinion in Neurobiology, vol. 22, no. 5, pp. 785-790, 2012.

[66] A. Y. L. Cheung, L. M. Horvath, L. Carrel, and J. Ellis, "Xchromosome inactivation in Rett syndrome human induced pluripotent stem cells," Frontiers in Psychiatry, vol. 3, article 24, 2012.
[67] P. E. Cundiff and S. A. Anderson, "Impact of induced pluripotent stem cells on the study of central nervous system disease," Current Opinion in Genetics and Development, vol. 21, no. 3, pp. 354-361, 2011.

[68] J. Duan, "Path from schizophrenia genomics to biology: gene regulation and perturbation in neurons derived from induced pluripotent stem cells and genome editing," Neuroscience Bulletin, vol. 31, no. 1, pp. 113-127, 2015.

[69] B. C. G. Freitas, C. A. Trujillo, C. Carromeu, M. Yusupova, R. H. Herai, and A. R. Muotri, "Stem cells and modeling of autism spectrum disorders," Experimental Neurology, vol. 260, pp. 3343, 2014.

[70] S.-M. Ho, A. Topol, and K. J. Brennand, "From 'directed differentiation' to 'neuronal induction': modeling neuropsychiatric disease," Biomarker Insights, vol. 10, supplement 1, pp. 31-41, 2015.

[71] G. Cocks, S. Curran, P. Gami et al., "The utility of patient specific induced pluripotent stem cells for the modelling of Autistic Spectrum Disorders," Psychopharmacology, vol. 231, no. 6, pp. 1079-1088, 2014

[72] K.-S. Kim, "Induced pluripotent stem (iPS) cells and their future in psychiatry," Neuropsychopharmacology, vol. 35, no. 1, pp. 346348, 2010.

[73] K.-Y. Kim, Y. W. Jung, G. J. Sullivan, L. Chung, and I.-H. Park, "Cellular reprogramming: a novel tool for investigating autism spectrum disorders," Trends in Molecular Medicine, vol. 18, no. 8, pp. 463-471, 2012.

[74] D.-S. Kim, P. Joel Ross, K. Zaslavsky, and J. Ellis, “Optimizing neuronal differentiation from induced pluripotent stem cells to model ASD," Frontiers in Cellular Neuroscience, vol. 8, no. 1, article 109, 2014.

[75] I. Ladran, N. Tran, A. Topol, and K. J. Brennand, "Neural stem and progenitor cells in health and disease," Wiley Interdisciplinary Reviews: Systems Biology and Medicine, vol. 5, no. 6, pp. 701-715, 2013.

[76] E. Y. Liu and C. T. Scott, "Great expectations: autism spectrum disorder and induced pluripotent stem cell technologies," Stem Cell Reviews and Reports, vol. 10, no. 2, pp. 145-150, 2014.

[77] A. Mackay-Sim, G. Mellick, and S. Wood, "Stem cell models for biomarker discovery in brain disease," International Review of Neurobiology, vol. 101, pp. 239-257, 2011.

[78] A. R. Muotri, "The human model: changing focus on autism research," Biological Psychiatry, 2015.

[79] K. S. O'Shea and M. G. McInnis, "Induced pluripotent stem cell (iPSC) models of bipolar disorder," Neuropsychopharmacology, vol. 40, no. 1, pp. 248-249, 2015.

[80] S. P. Paşca, G. Panagiotakos, and R. E. Dolmetsch, "Generating human neurons in vitro and using them to understand neuropsychiatric disease," Annual Review of Neuroscience, vol. 37, pp. 479-501, 2014.

[81] B. D. S. Paulsen, R. D. M. Maciel, A. Galina et al., "Altered oxygen metabolism associated to neurogenesis of induced pluripotent stem cells derived from a schizophrenic patient," Cell Transplantation, vol. 21, no. 7, pp. 1547-1559, 2012.

[82] B. D. S. Paulsen, M. S. da Silveira, A. Galina, and S. K. Rehen, "Pluripotent stem cells as a model to study oxygen metabolism in neurogenesis and neurodevelopmental disorders," Archives of Biochemistry and Biophysics, vol. 534, no. 1-2, pp. 3-10, 2013.

[83] D. Prilutsky, N. P. Palmer, N. Smedemark-Margulies, T. M. Schlaeger, D. M. Margulies, and I. S. Kohane, "iPSC-derived neurons as a higher-throughput readout for autism: promises 
and pitfalls," Trends in Molecular Medicine, vol. 20, no. 2, pp. 91-104, 2014.

[84] L. Qiang, K. Inoue, and A. Abeliovich, "Instant neurons: directed somatic cell reprogramming models of central nervous system disorders," Biological Psychiatry, vol. 75, no. 12, pp. 945951, 2014.

[85] E. E. Schadt, S. Buchanan, K. J. Brennand, and K. M. Merchant, "Evolving toward a human-cell based and multiscale approach to drug discovery for CNS disorders," Frontiers in Pharmacology, vol. 5, article 252, 2014.

[86] B. T. D. Tobe, M. G. Brandel, J. S. Nye, and E. Y. Snyder, "Implications and limitations of cellular reprogramming for psychiatric drug development," Experimental and Molecular Medicine, vol. 45, no. 11, article e59, 2013.

[87] N. N. Tran, I. G. Ladran, and K. J. Brennand, "Modeling schizophrenia using induced pluripotent stem cell-derived and fibroblast-induced neurons," Schizophrenia Bulletin, vol. 39, no. 1, pp. 4-10, 2013.

[88] F. M. Vaccarino, A. E. Urban, H. E. Stevens et al., "Annual research review: the promise of stem cell research for neuropsychiatric disorders," Journal of Child Psychology and Psychiatry, vol. 52, no. 4, pp. 504-516, 2011.

[89] B. Viswanath, S. P. Jose, A. Squassina et al., "Cellular models to study bipolar disorder: a systematic review," Journal of Affective Disorders, vol. 184, pp. 36-50, 2015.

[90] R. M. Walsh and K. Hochedlinger, "Modeling Rett syndrome with stem cells," Cell, vol. 143, no. 4, pp. 499-500, 2010.

[91] R. Wright, J. M. Réthelyi, and F. H. Gage, "Enhancing induced pluripotent stem cell models of schizophrenia," JAMA Psychiatry, vol. 71, no. 3, pp. 334-335, 2014.

[92] K. G. Chen, B. S. Mallon, R. D. G. McKay, and P. G. Robey, "Human pluripotent stem cell culture: considerations for maintenance, expansion, and therapeutics," Cell Stem Cell, vol. 14, no. 1, pp. 13-26, 2014.

[93] S. H. Yuan and M. Shaner, "Bioengineered stem cells in neural development and neurodegeneration research," Ageing Research Reviews, vol. 12, no. 3, pp. 739-748, 2013.

[94] S.-M. Ho, A. Topol, and K. J. Brennand, "From 'directed differentiation' to 'neuronal induction': modeling neuropsychiatric disease," Biomarker Insights, vol. 10, supplement 1, pp. 31-41, 2015.

[95] T. Vierbuchen, A. Ostermeier, Z. P. Pang, Y. Kokubu, T. C. Südhof, and M. Wernig, "Direct conversion of fibroblasts to functional neurons by defined factors," Nature, vol. 463, no. 7284, pp. 1035-1041, 2010.

[96] A. S. Yoo, A. X. Sun, L. Li et al., "MicroRNA-mediated conversion of human fibroblasts to neurons," Nature, vol. 476, no. 7359, pp. 228-231, 2011.

[97] C. Compagnucci, M. Nizzardo, S. Corti, G. Zanni, and E. Bertini, "In vitro neurogenesis: development and functional implications of iPSC technology," Cellular and Molecular Life Sciences, vol. 71, no. 9, pp. 1623-1639, 2014.

[98] K. Takahashi and S. Yamanaka, "Induction of pluripotent stem cells from mouse embryonic and adult fibroblast cultures by defined factors," Cell, vol. 126, no. 4, pp. 663-676, 2006.

[99] J. Yu, M. A. Vodyanik, K. Smuga-Otto et al., "Induced pluripotent stem cell lines derived from human somatic cells," Science, vol. 318, no. 5858, pp. 1917-1920, 2007.

[100] X. Y. Deng, H. Wang, T. Wang et al., "Non-viral methods for generating integration-free, induced pluripotent stem cells," Current Stem Cell Research \& Therapy, vol. 10, no. 2, pp. 153-158, 2015.
[101] S. M. Chambers, C. A. Fasano, E. P. Papapetrou, M. Tomishima, M. Sadelain, and L. Studer, "Highly efficient neural conversion of human ES and iPS cells by dual inhibition of SMAD signaling," Nature Biotechnology, vol. 27, no. 3, pp. 275-280, 2009.

[102] H. Zeng, M. Guo, K. Martins-Taylor et al., "Specification of region-specific neurons including forebrain glutamatergic neurons from human induced pluripotent stem cells," PLoS ONE, vol. 5, no. 7, Article ID el1853, 2010.

[103] C. R. Muratore, P. Srikanth, D. G. Callahan, T. L. YoungPearse, and M. Dottori, "Comparison and optimization of hiPSC forebrain cortical differentiation protocols," PLOS ONE, vol. 9, no. 8, Article ID e105807, 2014.

[104] Y. Liu, H. Liu, C. Sauvey, L. Yao, E. D. Zarnowska, and S.-C. Zhang, "Directed differentiation of forebrain GABA interneurons from human pluripotent stem cells," Nature Protocols, vol. 8, no. 9, pp. 1670-1679, 2013.

[105] A. M. Maroof, S. Keros, J. A. Tyson et al., "Directed differentiation and functional maturation of cortical interneurons from human embryonic stem cells," Cell Stem Cell, vol. 12, no. 5, pp. 559-572, 2013.

[106] L. F. Boyer, B. Campbell, S. Larkin, Y. Mu, and F. H. Gage, "Dopaminergic differentiation of human pluripotent cells," Current Protocols in Stem Cell Biology, 2012.

[107] A. Sánchez-Danés, A. Consiglio, Y. Richaud et al., "Efficient generation of A9 midbrain dopaminergic neurons by lentiviral delivery of LMX1A in human embryonic stem cells and induced pluripotent stem cells," Human Gene Therapy, vol. 23, no. 1, pp. 56-69, 2012.

[108] J. Mariani, M. V. Simonini, D. Palejev et al., "Modeling human cortical development in vitro using induced pluripotent stem cells," Proceedings of the National Academy of Sciences of the United States of America, vol. 109, no. 31, pp. 12770-12775, 2012.

[109] D. Pamies, T. Hartung, and H. T. Hogberg, "Biological and medical applications of a brain-on-a-chip," Experimental Biology and Medicine, vol. 239, no. 9, pp. 1096-1107, 2014.

[110] L. D’Aiuto, Y. Zhi, D. Kumar Das et al., "Large-scale generation of human iPSC-derived neural stem cells/early neural progenitor cells and their neuronal differentiation," Organogenesis, vol. 10, no. 4, pp. 365-377, 2014.

[111] K. E. Santostefano, T. Hamazaki, N. M. Biel, S. Jin, A. Umezawa, and N. Terada, "A practical guide to induced pluripotent stem cell research using patient samples," Laboratory Investigation, vol. 95, no. 1, pp. 4-13, 2015.

[112] K. Martins-Taylor, B. S. Nisler, S. M. Taapken et al., "Recurrent copy number variations in human induced pluripotent stem cells," Nature Biotechnology, vol. 29, no. 6, pp. 488-491, 2011.

[113] World Health Organization, Global Health Estimates 2014 Summary Tables: DALY by Cause, Age and Sex, WHO Region, 20002012.

[114] J. R. Geddes, S. M. Carney, C. Davies et al., "Relapse prevention with antidepressant drug treatment in depressive disorders: a systematic review," The Lancet, vol. 361, no. 9358, pp. 653-661, 2003.

[115] F. W. Lohoff, "Overview of the genetics of major depressive disorder," Current Psychiatry Reports, vol. 12, no. 6, pp. 539-546, 2010.

[116] D. H. Manier, R. C. Shelton, T. C. Ellis, C. S. Peterson, A. Eiring, and F. Sulser, "Human fibroblasts as a relevant model to study signal transduction in affective disorders," Journal of Affective Disorders, vol. 61, no. 1-2, pp. 51-58, 2000. 
[117] D. Martins-De-souza, G. Maccarrone, M. Ising et al., "Blood mononuclear cell proteome suggests integrin and Ras signaling as critical pathways for antidepressant treatment response," Biological Psychiatry, vol. 76, no. 7, pp. e15-e17, 2014.

[118] A. R. Tyrka, S. H. Parade, L. H. Price et al., "Alterations of mitochondrial DNA copy number and telomere length with early adversity and psychopathology," Biological Psychiatry, 2015.

[119] V. Broccoli, S. G. Giannelli, and P. G. Mazzara, "Modeling physiological and pathological human neurogenesis in the dish," Frontiers in Neuroscience, vol. 8, article 183, 2014.

[120] M. Song, O. Mohamad, D. Chen, and S. P. Yu, "Coordinated development of voltage-gated $\mathrm{Na}^{+}$and $\mathrm{K}^{+}$currents regulates functional maturation of forebrain neurons derived from human induced pluripotent stem cells," Stem Cells and Development, vol. 22, no. 10, pp. 1551-1563, 2013.

[121] C. Verpelli, L. Carlessi, G. Bechi et al., "Comparative neuronal differentiation of self-renewing neural progenitor cell lines obtained from human induced pluripotent stem cells," Frontiers in Cellular Neuroscience, vol. 7, article 175, 2013.

[122] S. Wang, B. Wang, N. Pan et al., "Differentiation of human induced pluripotent stem cells to mature functional Purkinje neurons," Scientific Reports, vol. 5, article 9232, 2015.

[123] A. Fathi, M. Hatami, V. Hajihosseini et al., "Comprehensive gene expression analysis of human embryonic stem cells during differentiation into neural cells," PLoS ONE, vol. 6, no. 7, Article ID e22856, 2011.

[124] X. Tang, L. Zhou, A. M. Wagner et al., "Astroglial cells regulate the developmental timeline of human neurons differentiated from induced pluripotent stem cells," Stem Cell Research, vol. 11, no. 2, pp. 743-757, 2013.

[125] G. Meneghello, A. Verheyen, M. Van Ingen et al., "Evaluation of established human iPSC-derived neurons to model neurodegenerative diseases," Neuroscience, vol. 301, pp. 204-212, 2015.

[126] F. Soldner, D. Hockemeyer, C. Beard et al., "Parkinson's disease patient-derived induced pluripotent stem cells free of viral reprogramming factors," Cell, vol. 136, no. 5, pp. 964-977, 2009.

[127] D. Yang, T. Li, M. Xu et al., "Graphene oxide promotes the differentiation of mouse embryonic stem cells to dopamine neurons," Nanomedicine, vol. 9, no. 16, pp. 2445-2455, 2014.

[128] L. C. Jansson and K. E. Åkerman, "The role of glutamate and its receptors in the proliferation, migration, differentiation and survival of neural progenitor cells," Journal of Neural Transmission, vol. 121, no. 8, pp. 819-836, 2014.

[129] F. Wegner, R. Kraft, K. Busse et al., "Functional and molecular analysis of $\mathrm{GABA}_{\mathrm{A}}$ receptors in human midbrain-derived neural progenitor cells," Journal of Neurochemistry, vol. 107, no. 4, pp. 1056-1069, 2008.

[130] C. L. Lao, C.-S. Lu, and J.-C. Chen, "Dopamine D3 receptor activation promotes neural stem/progenitor cell proliferation through AKT and ERK1/2 pathways and expands type-B and -C cells in adult subventricular zone," Glia, vol. 61, no. 4, pp. 475-489, 2013.

[131] E. M. Hartfield, M. Yamasaki-Mann, H. J. Ribeiro Fernandes et al., "Physiological characterisation of human iPS-derived dopaminergic neurons," PLoS ONE, vol. 9, no. 2, Article ID e87388, 2014.

[132] A. Chatzidaki, A. Fouillet, J. Li et al., "Pharmacological characterisation of nicotinic acetylcholine receptors expressed in human iPSC-derived neurons," PLOS ONE, vol.10, no. 4, Article ID e0125116, 2015.
[133] A. Citri, Z. P. Pang, T. C. Südhof, M. Wernig, and R. C. Malenka, "Comprehensive qPCR profiling of gene expression in single neuronal cells," Nature Protocols, vol. 7, no. 1, pp. 118-127, 2011.

[134] Z. P. Pang, N. Yang, T. Vierbuchen et al., "Induction of human neuronal cells by defined transcription factors," Nature, vol. 476, no. 7359, pp. 220-223, 2011.

[135] G. M. Khandaker and R. Dantzer, "Is there a role for immuneto-brain communication in schizophrenia?" Psychopharmacology, 2015.

[136] L. L. Hurley and Y. Tizabi, "Neuroinflammation, neurodegeneration, and depression," Neurotoxicity Research, vol. 23, no. 2, pp. 131-144, 2013.

[137] C. Möller and M. Slack, "Impact of new technologies for cellular screening along the drug value chain," Drug Discovery Today, vol. 15, no. 9-10, pp. 384-390, 2010.

[138] M. Kaufmann, A. Schuffenhauer, I. Fruh et al., "Highthroughput screening using iPSC-derived neuronal progenitors to identify compounds counteracting epigenetic gene silencing in fragile X Syndrom," Journal of Biomolecular Screening, vol. 20, no. 9, pp. 1101-1111, 2015.

[139] A. Efthymiou, A. Shaltouki, J. P. Steiner et al., "Functional screening assays with neurons generated from pluripotent stem cell-derived neural stem cells," Journal of Biomolecular Screening, vol. 19, no. 1, pp. 32-43, 2014.

[140] S. M. Taapken, B. S. Nisler, M. A. Newton et al., "Karotypic abnormalities in human induced pluripotent stem cells and embryonic stem cells," Nature Biotechnology, vol. 29, no. 4, pp. 313-314, 2011.

[141] H. Mkrtchyan, M. Gross, S. Hinreiner et al., "The human genome puzzle-the role of copy number variation in somatic mosaicism," Current Genomics, vol. 11, no. 6, pp. 426-431, 2010.

[142] S. M. Hussein, N. N. Batada, S. Vuoristo et al., "Copy number variation and selection during reprogramming to pluripotency," Nature, vol. 471, no. 7336, pp. 58-62, 2011.

[143] D. Grozeva, D. F. Conrad, C. P. Barnes et al., "Independent estimation of the frequency of rare CNVs in the UK population confirms their role in schizophrenia," Schizophrenia Research, vol. 135, no. 1-3, pp. 1-7, 2012.

[144] J.-A. Ramos-Quiroga, C. Sánchez-Mora, M. Casas et al., "Genome-wide copy number variation analysis in adult attention-deficit and hyperactivity disorder," Journal of Psychiatric Research, vol. 49, no. 1, pp. 60-67, 2014.

[145] X. Kang, Q. Yu, Y. Huang et al., "Effects of integrating and nonintegrating reprogramming methods on copy number variation and genomic stability of human induced pluripotent stem cells," PLOS ONE, vol. 10, no. 7, Article ID e0131128, 2015.

[146] A. Poduri, G. D. Evrony, X. Cai, and C. A. Walsh, "Somatic mutation, genomic variation, and neurological disease," Science, vol. 341, no. 6141, Article ID 1237758, 2013.

[147] C. A. Thomas, A. C. M. Paquola, and A. R. Muotri, "LINE-1 retrotransposition in the nervous system," Annual Review of Cell and Developmental Biology, vol. 28, pp. 555-573, 2012.

[148] S. Eminli, A. Foudi, M. Stadtfeld et al., "Differentiation stage determines potential of hematopoietic cells for reprogramming into induced pluripotent stem cells," Nature Genetics, vol. 41, no. 9, pp. 968-976, 2009.

[149] A. Banito, S. T. Rashid, J. C. Acosta et al., "Senescence impairs successful reprogramming to pluripotent stem cells," Genes \& Development, vol. 23, no. 18, pp. 2134-2139, 2009.

[150] K. Streckfuss-Bömeke, F. Wolf, A. Azizian et al., "Comparative study of human-induced pluripotent stem cells derived from 
bone marrow cells, hair keratinocytes, and skin fibroblasts," European Heart Journal, vol. 34, no. 33, pp. 2618-2629, 2013.

[151] R. Trokovic, J. Weltner, P. Noisa, T. Raivio, and T. Otonkoski, "Combined negative effect of donor age and time in culture on the reprogramming efficiency into induced pluripotent stem cells," Stem Cell Research, vol. 15, no. 1, pp. 254-262, 2015.

[152] Z. Ghosh, K. D. Wilson, Y. Wu, S. Hu, T. Quertermous, and J. C. Wu, "Persistent donor cell gene expression among human induced pluripotent stem cells contributes to differences with human embryonic stem cells," PLoS ONE, vol. 5, no. 2, article e8975, 2010.

[153] American Psychiatric Association, Diagnostic and Statistical Manual of Mental Disorders: DSM-5U゚, American Psychiatric Association, Arlington, Va, USA, 5th edition, 2013.

[154] D. L. Braff, "The importance of endophenotypes in schizophrenia research," Schizophrenia Research, vol. 163, no. 1-3, pp. 1-8, 2015.

[155] L. J. Seidman, G. Hellemann, K. H. Nuechterlein et al., "Factor structure and heritability of endophenotypes in schizophrenia: findings from the Consortium on the Genetics of Schizophrenia (COGS-1)," Schizophrenia Research, vol. 163, no. 1-3, pp. 73-79, 2015.

[156] D. St Clair, D. Blackwood, W. Muir et al., "Association within a family of a balanced autosomal translocation with major mental illness," The Lancet, vol. 336, no. 8706, pp. 13-16, 1990.

[157] P. A. Thomson, E. L. V. Malavasi, E. Grünewald, D. C. Soares, M. Borkowska, and J. K. Millar, "DISC1 genetics, biology and psychiatric illness," Frontiers in Biology, vol. 8, no. 1, pp. 1-31, 2013.

[158] U. Grieshammer and K. A. Shepard, "Proceedings: consideration of genetics in the design of induced pluripotent stem cell-based models of complex disease," Stem Cells Translational Medicine, vol. 3, no. 11, pp. 1253-1258, 2014.

[159] D. Hockemeyer, H. Wang, S. Kiani et al., "Genetic engineering of human pluripotent cells using TALE nucleases," Nature Biotechnology, vol. 29, no. 8, pp. 731-734, 2011.

[160] D. Hockemeyer, F. Soldner, C. Beard et al., "Efficient targeting of expressed and silent genes in human ESCs and iPSCs using zinc-finger nucleases," Nature Biotechnology, vol. 27, no. 9, pp. 851-857, 2009.

[161] T. P. Zwaka and J. A. Thomson, "Homologous recombination in human embryonic stem cells," Nature Biotechnology, vol. 21, no. 3, pp. 319-321, 2003.

[162] S. W. Cho, S. Kim, J. M. Kim, and J.-S. Kim, “Targeted genome engineering in human cells with the Cas9 RNA-guided endonuclease," Nature Biotechnology, vol. 31, no. 3, pp. 230-232, 2013.

[163] A. A. Sproul, "Being human: the role of pluripotent stem cells in regenerative medicine and humanizing Alzheimer's disease models," Molecular Aspects of Medicine, vol. 43-44, pp. 54-65, 2015.

[164] C. Gonzalez, S. Bonilla, A. I. Flores, E. Cano, and I. Liste, "An update on human stem cell-based therapy in Parkinson's disease," Current Stem Cell Research \& Therapy, In press.

[165] D. J. Lodge and A. A. Grace, "Aberrant hippocampal activity underlies the dopamine dysregulation in an animal model of schizophrenia," Journal of Neuroscience, vol. 27, no. 42, pp. 11424-11430, 2007.

[166] T.-G. Kim, R. Yao, T. Monnell et al., "Efficient specification of interneurons from human pluripotent stem cells by dorsoventral and rostrocaudal modulation," Stem Cells, vol. 32, no. 7, pp. 1789-1804, 2014.
[167] F.-W. Zhou, J. M. Fortin, H.-X. Chen et al., "Functional integration of human neural precursor cells in mouse cortex," PLoS ONE, vol. 10, no. 3, Article ID e0120281, 2015.

[168] S. M. Perez and D. J. Lodge, "Hippocampal interneuron transplants reverse aberrant dopamine system function and behavior in a rodent model of schizophrenia," Molecular Psychiatry, vol. 18, no. 11, pp. 1193-1198, 2013.

[169] http://eur-lex.europa.eu/legal-content/EN/TXT/PDF/?uri=CELEX:32012H0417\&rid=1.

[170] http://www.sparc.arl.org/advocacy/national/fastr.

[171] D. Murphy and W. Spooren, "EU-AIMS: a boost to autism research," Nature Reviews Drug Discovery, vol. 11, no. 11, pp. 815816,2012

[172] S. Bölte, C. Willfors, S. Berggren et al., "The roots of Autism and ADHD twin study in Sweden (RATSS)," Twin Research and Human Genetics, vol. 17, no. 3, pp. 164-176, 2014.

[173] J. Genius, A. Schellenberg, L. Tchana-Duope et al., "Enhanced calcium responses to serotonin receptor stimulation in Tlymphocytes from schizophrenic patients-a pilot study," Neuroscience Letters, vol. 589, pp. 159-162, 2015.

[174] J.-I. Iga, S.-I. Ueno, and T. Ohmori, "Molecular assessment of depression from mRNAs in the peripheral leukocytes," Annals of Medicine, vol. 40, no. 5, pp. 336-342, 2008.

[175] D. Akin, D. H. Manier, E. Sanders-Bush, and R. C. Shelton, "Signal transduction abnormalities in melancholic depression," International Journal of Neuropsychopharmacology, vol. 8, no. 1, pp. 5-16, 2005.

[176] S. Hänzelmann, F. Beier, E. G. Gusmao et al., "Replicative senescence is associated with nuclear reorganization and with DNA methylation at specific transcription factor binding sites," Clinical Epigenetics, vol. 7, no. 1, article 19, 2015.

[177] K. Krieger, A. Klimke, and U. Henning, "Active $\left[{ }^{3} \mathrm{H}\right]$-dopamine uptake displayed by native lymphocyte suspensions is mainly due to contaminating platelets," Pharmacopsychiatry, vol. 31, no. 5, pp. 193-198, 1998.

[178] J. H. Lee, R. R. Mitchell, J. D. McNicol et al., "Single transcription factor conversion of human blood fate to NPCs with CNS and PNS developmental capacity," Cell Reports, vol. 11, no. 9, pp. 1367-1376, 2015.

[179] S. Marro, Z. P. Pang, N. Yang et al., "Direct lineage conversion of terminally differentiated hepatocytes to functional neurons," Cell Stem Cell, vol. 9, no. 4, pp. 374-382, 2011.

[180] Q. Zou, Q. Yan, J. Zhong et al., "Direct conversion of human fibroblasts into neuronal restricted progenitors," The Journal of Biological Chemistry, vol. 289, no. 8, pp. 5250-5260, 2014.

[181] S. Chanda, C. E. Ang, J. Davila et al., "Generation of induced neuronal cells by the single reprogramming factor ASCL1," Stem Cell Reports, vol. 3, no. 2, pp. 282-296, 2014.

[182] M. Caiazzo, M. T. Dell'Anno, E. Dvoretskova et al., "Direct generation of functional dopaminergic neurons from mouse and human fibroblasts," Nature, vol. 476, no. 7359, pp. 224-227, 2011.

[183] E. Y. Son, J. K. Ichida, B. J. Wainger et al., "Conversion of mouse and human fibroblasts into functional spinal motor neurons," Cell Stem Cell, vol. 9, no. 3, pp. 205-218, 2011.

[184] F. J. Najm, A. M. Lager, A. Zaremba et al., "Transcription factor-mediated reprogramming of fibroblasts to expandable, myelinogenic oligodendrocyte progenitor cells," Nature Biotechnology, vol. 31, no. 5, pp. 426-433, 2013.

[185] S. Kelaini, A. Cochrane, and A. Margariti, "Direct reprogramming of adult cells: avoiding the pluripotent state," Stem Cells 
and Cloning: Advances and Applications, vol. 7, no. 1, pp. 19-29, 2014.

[186] B. M. Morrison, Y. Lee, and J. D. Rothstein, "Oligodendroglia: metabolic supporters of axons," Trends in Cell Biology, vol. 23, no. 12, pp. 644-651, 2013.

[187] M. Bélanger, I. Allaman, and P. J. Magistretti, "Brain energy metabolism: focus on astrocyte-neuron metabolic cooperation," Cell Metabolism, vol. 14, no. 6, pp. 724-738, 2011.

[188] R. D. Fields, D. H. Woo, and P. J. Basser, "Glial regulation of the neuronal connectome through local and long-distant communication," Neuron, vol. 86, no. 2, pp. 374-386, 2015.

[189] M. Santello, C. Calì, and P. Bezzi, "Gliotransmission and the tripartite synapse," Advances in Experimental Medicine and Biology, vol. 970, pp. 307-331, 2012.

[190] C. Eroglu and B. A. Barres, "Regulation of synaptic connectivity by glia," Nature, vol. 468, no. 7321, pp. 223-231, 2010.

[191] G. Sanacora and M. Banasr, "From pathophysiology to novel antidepressant drugs: glial contributions to the pathology and treatment of mood disorders," Biological Psychiatry, vol. 73, no. 12, pp. 1172-1179, 2013.

[192] R. Krencik and S.-C. Zhang, "Directed differentiation of functional astroglial subtypes from human pluripotent stem cells," Nature Protocols, vol. 6, no. 11, pp. 1710-1717, 2011.

[193] M. Czepiel, V. Balasubramaniyan, W. Schaafsma et al., "Differentiation of induced pluripotent stem cells into functional oligodendrocytes," Glia, vol. 59, no. 6, pp. 882-892, 2011.

[194] M. Ohgidani, T. A. Kato, D. Setoyama et al., "Direct induction of ramified microglia-like cells from human monocytes: dynamic microglial dysfunction in Nasu-Hakola disease," Scientific Reports, vol. 4, article 4957, 2014.

[195] T. A. Juopperi, W. R. Kim, C.-H. Chiang et al., "Astrocytes generated from patient induced pluripotent stem cells recapitulate features of Huntington's disease patient cells," Molecular Brain, vol. 5, no. 1, article 17, 2012.

[196] A. Serio, B. Bilican, S. J. Barmada et al., "Astrocyte pathology and the absence of non-cell autonomy in an induced pluripotent stem cell model of TDP-43 proteinopathy," Proceedings of the National Academy of Sciences of the United States of America, vol. 110, no. 12, pp. 4697-4702, 2013.

[197] S. Wang, J. Bates, X. Li et al., "Human iPSC-derived oligodendrocyte progenitor cells can myelinate and rescue a mouse model of congenital hypomyelination," Cell Stem Cell, vol. 12, no. 2, pp. 252-264, 2013.

[198] R. Krencik, K. C. Hokanson, A. R. Narayan et al., "Dysregulation of astrocyte extracellular signaling in Costello syndrome," Science Translational Medicine, vol. 7, no. 286, Article ID 286ra66, 2015. 